
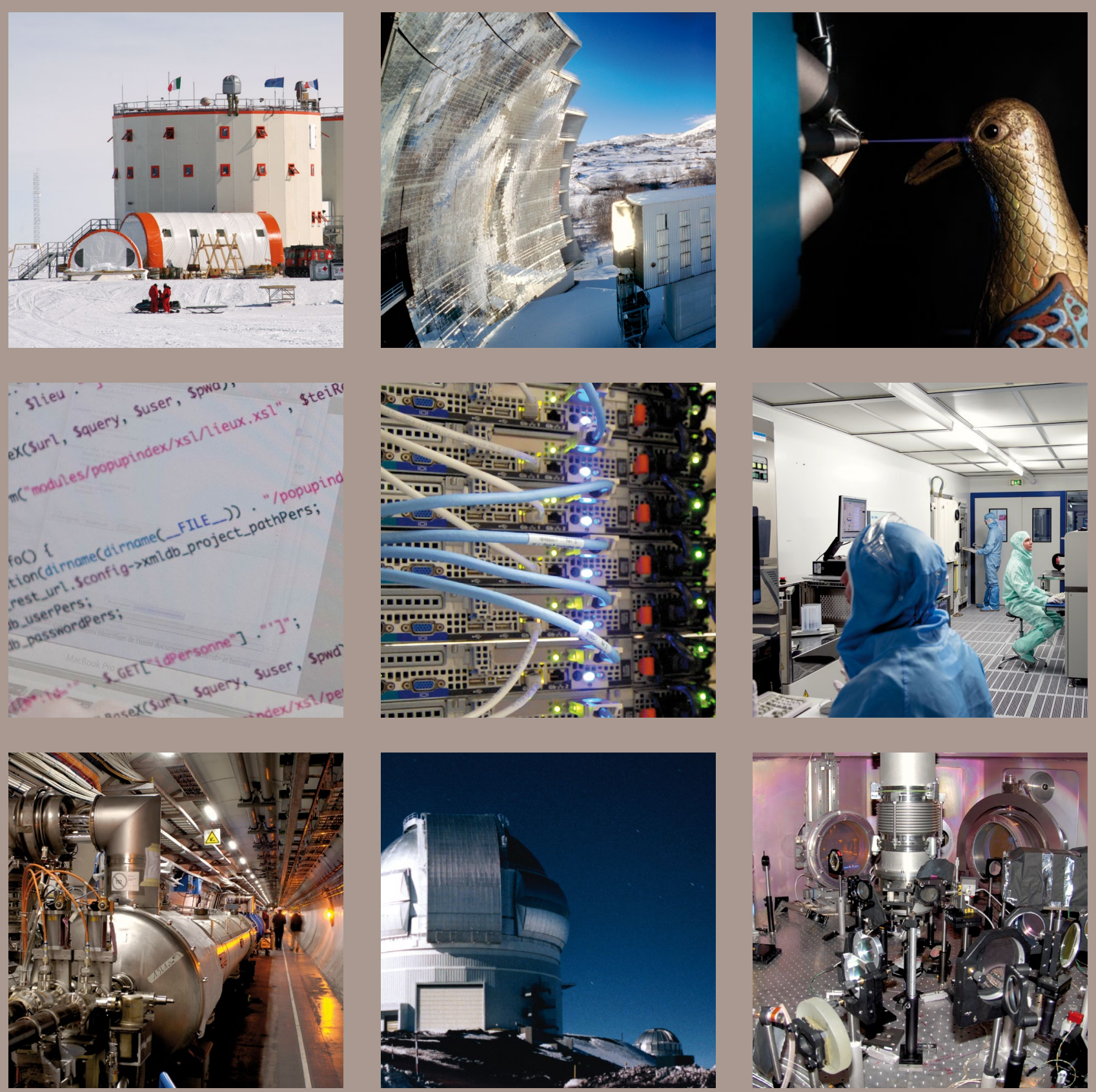

\title{
STRATÉGIE NATIONALE
}

DES INFRASTRUCTURES DE RECHERCHE

ÉDITION 2016

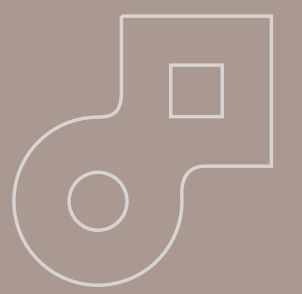

www.enseignementsup-recherche.gouv.fr

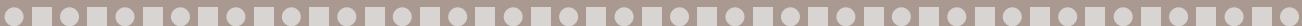

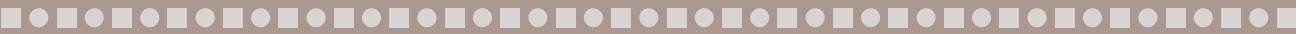

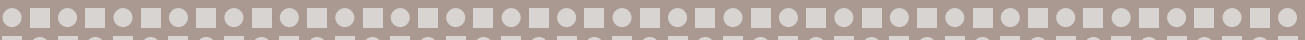

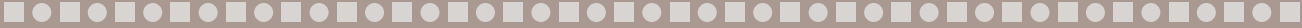

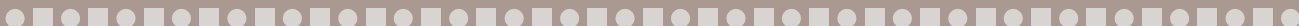

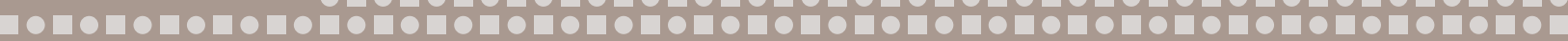

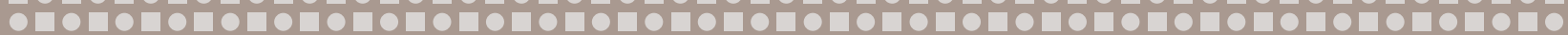

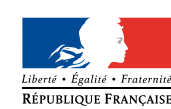

-

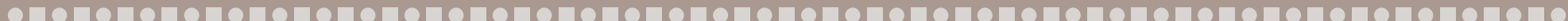

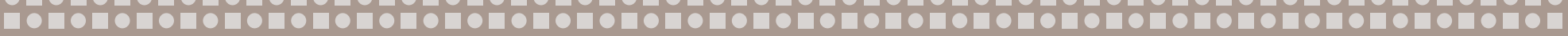



Thierry MANDON

Secrétaire d'État chargé de

I'Enseignement supérieur et de la Recherche

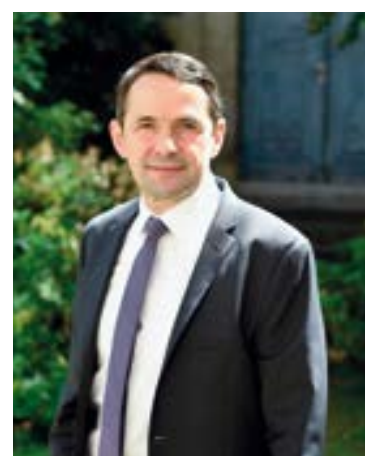

Les mesures annoncées à l'issue de la COP21 qui s'est tenue à Paris en décembre 2015 sont porteuses, pour notre avenir, d'une belle note d'espoir. Ces résultats, s'ils doivent beaucoup à la ténacité des négociateurs, proviennent plus profondément d'un processus de maturation dans la prise de conscience des impacts du changement climatique pour chaque pays présent. Cette maturation est largement due aux travaux des scientifiques qui, depuis vingt ans, ont inlassablement amélioré leurs observations et affiné leurs résultats jusqu'à remporter un large consensus sur le caractère anthropique du changement climatique. Si les scientifiques ont obtenu ces résultats, c'est qu'ils ont peu à peu disposé des outils de mesure, d'observation, de modélisation et de simulation appropriés à la complexité des problèmes à résoudre.

Des outils que nous appelons des infrastructures de recherche (IR) ou des «très grandes infrastructures de recherche (TGIR) ». Autrement dit, des moyens de recherche dont la durée de vie peut se compter en décennies et dont l'impact socioéconomique local ou national est très prégnant. Les TGIR nécessitent, pour les construire et les mettre en œuvre, des technologies nouvelles, des emplois hautement qualifiés et ce que cela suppose en termes de formation, et d'importants moyens financiers qui demandent une coopération à l'échelle européenne, voire internationale.

Là se trouve la raison d'être de la feuille de route des IR nationales, européennes ou internationales : disposer d'une vision maîtrisée de ces investissements particulièrement lourds. La France a été pionnière en matière de programmation pluriannuelle et de pilotage de ces moyens de recherche, par ses établissements de recherche et son Ministère chargé de la recherche.
En Europe, les Etats se sont rassemblés au sein du forum ESFRI (European Scientific Forum for Research Infrastructures). La France qui a participé activement, dans le cadre de l'ESFRI, à la mise à jour de la feuille de route des infrastructures de recherche européennes officiellement publiée en mars 2016, a également revisité, en parallèle, la feuille de route de ses installations nationales.

Ce processus a fait l'objet d'une intense activité au cours de l'année 2015 , en partenariat étroit avec les établissements et les Alliances de recherche, à partir d'une analyse par grands secteurs de recherche que l'on retrouve dans les chapitres de ce document, accompagnée d'une stratégie d'orientation pour les années à venir.

Tout ceci a débouché sur une liste d'Infrastructures de recherche, qui constitue un véritable tableau de bord, permettant à l'Etat de disposer d'une base solide pour des plans d'action pluriannuels.

Aujourd'hui, nous en mesurons le résultat: de natures très variées - mais avec des caractéristiques communes, rappelées dans ce document, les infrastructures françaises révèlent le large spectre d'outils et d'équipements nécessaires au développement des grands secteurs scientifiques. Elles sont un atout majeur de notre pays pour maintenir sa place dans la compétition mondiale et le Gouvernement œuvrera pour maintenir un effort financier à la hauteur des enjeux.

II me reste à remercier et à féliciter l'ensemble des acteurs de la communauté scientifique, des institutions de recherche et des équipes ministérielles pour leur travail exemplaire de coordination, leur capacité à s'auto-organiser et à prendre ensemble des décisions communes au bénéfice des intérêts du pays. 
Plus que jamais, les enjeux scientifiques posent le défi de construire des outils de recherche à la pointe des connaissances scientifiques et technologiques. Les frontières de la connaissance ont reculé jusqu'à des extrêmes que seules des réalisations technologiques majeures permettent de confronter à la réalité expérimentale.

L'observation, la mesure, l'expérimentation, le calcul intensif, le stockage et le partage de données supposent de grands instruments portant les capacités techniques au-delà de l'existant et intégrant la porosité interdisciplinaire source d'innovation. Ces outils sont les conditions des futures découvertes tout autant que le produit des dernières avancées scientifiques et technologiques. De grands équipements ont été ainsi créés, pilotés par des organisations nationales, européennes ou internationales, nécessitant une instrumentation de premier plan mais aussi des ressources humaines et financières conséquentes, grâce au soutien de la puissance publique.

Parallèlement à ces grands programmes, se sont développés ces dernières années une quantité d'instruments partagés entre de nombreux acteurs sur des sites divers : nouveaux modes de microscopie et d'imagerie, nouveaux dispositifs de criblage à haut débit, expériences virtuelles, bases de données sociales, environnementales et de santé, corpus de textes numérisés enrichis d'outils d'exploitation... En France, le soutien du Programme Investissements d'Avenir a été essentiel à ce succès.

Dans ce contexte, une stratégie nationale de développement des infrastructures s'impose. Il convient de prendre en compte les nouveaux apports de la technologie, l'évolution des pratiques scientifiques, l'articulation dans des réseaux européens ou internationaux, les investissements humains et financiers, les retombées pour l'innovation tout comme les reconfigurations du paysage français de la recherche.

L'édition 2016 de la stratégie nationale sur les infrastructures de recherche témoigne de la volonté de l'Etat, par ses grands établissements de recherche et d'enseignement supérieur, de répondre aux exigences de savoir et d'innovation. Elaborée à partir d'un état de l'art proposé par des scientifiques de tous les domaines en articulation avec la Stratégie Nationale de Recherche, cette feuille de route est l'occasion de mettre en avant une diversité d'instruments, déjà existants ou en projet, qui doivent tous répondre aux mêmes critères d'excellence scientifique et technologique, d'efficacité et de transparence de la gouvernance, et d'ouverture effective à une communauté large. Au-delà de l'accès, les infrastructures de recherche génératrices de masse de données en quantité exponentiellement croissante sont attendues sur leur capacité à les rendre accessibles à l'ensemble de la communauté scientifique et, au-delà, à l'ensemble de la société. Il s'agit là d'un des grands défis à relever dans les années à venir. Ces infrastructures doivent enfin consolider leur modèle économique incluant une analyse budgétaire complète, un positionnement dans l'écosystème de l'innovation et l'identification de leurs interactions avec les acteurs du monde socio-économique.

Ce document présente des dispositifs existants qui constituent une réelle "force de frappe " au cœur de la stratégie nationale de recherche, ainsi que des projets formant une base pour la construction du futur. 


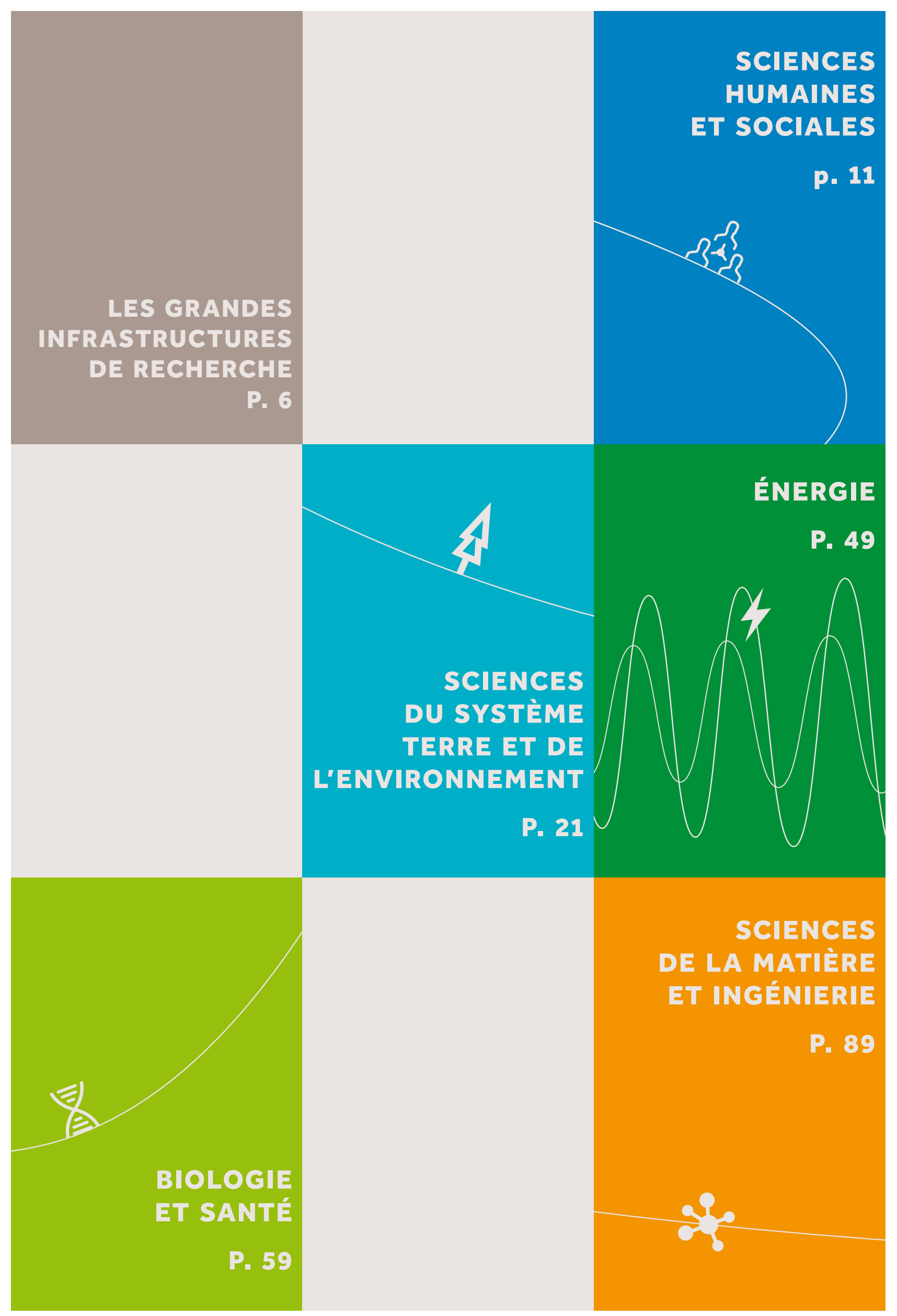


ASTRONOMIE ET ASTROPHYSIQUE

P. 109

SCIENCES ET TECHNOLOGIES DU NUMÉRIQU: ; MATHÉMATIQUES

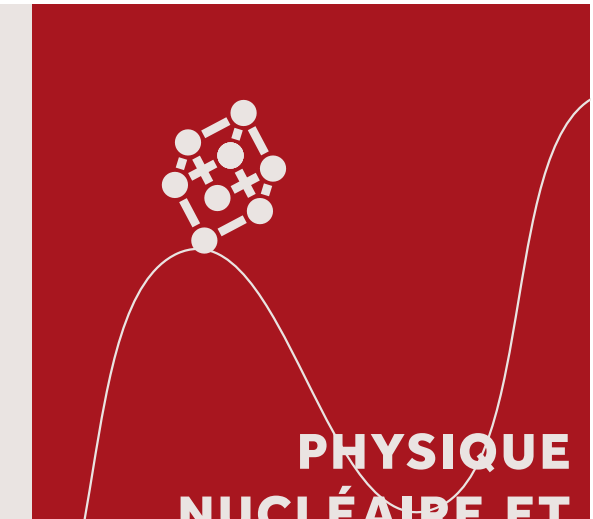
NUCLÉAYRE ET DES HAUTES ÉNERGIES

P. 125

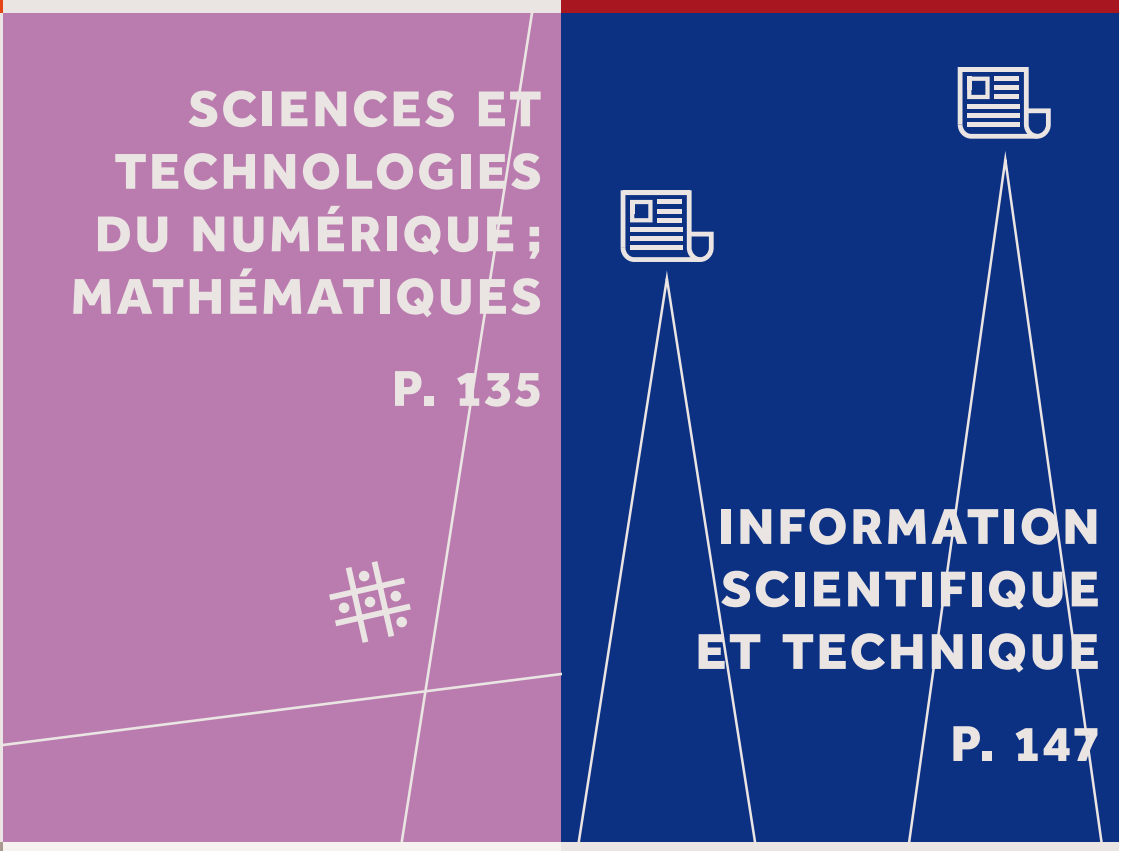

ANNEXES

P. 157 


\section{LES DIFFÉRENTS TYPES \\ DE GRANDES INFRASTRUCTURES \\ DE RECHERCHE}

\section{UN PAYSAGE RICHE ET VARIÉ}

La feuille de route nationale 2016 a retenu 95 infrastructures, dont les formes et les contenus sont extrêmement variés. Elles ne se limitent pas aux seuls grands appareils implantés sur un seul site, mais prennent également des formes distribuées pour être au plus près des communautés scientifiques. Elles sont également, à des degrés divers, influencées par les nouvelles capacités issues des technologies de l'information et de la communication. Elles traduisent enfin des modes d'organisation fortement dépendantes des communautés thématiques et des techniques qu'elles partagent. Quatre formes peuvent être identifiées :

- sur un seul site : les infrastructures localisées, le plus souvent du fait d'une instrumentation de grande taille nécessitant un programme immobilier spécifique;

- distribuée : les flottes, les réseaux de sites instrumentés ou de plateformes, les collections, archives et bibliothèques scientifiques;

- dématérialisée : les infrastructures de recherche virtuelles, les bases de données;

- les infrastructures à la base de réseaux humains (cohortes, experts, etc).

\section{QU'EST-CE QU'UNE GRANDE INFRASTRUCTURE DE RECHERCHE?}

Les principes définissant une grande infrastructure de recherche peuvent s'énoncer ainsi :

- elle doit être un outil ou un dispositif possédant des caractéristiques uniques identifiées par la communauté scientifique utilisatrice comme requises pour la conduite d'activités de recherche de haut niveau. Les communautés scientifiques visées peuvent être nationales, européennes ou internationales, selon le cas;

- elle doit disposer d'une gouvernance identifiée, unifiée et effective, et d'instances de pilotage stratégique et scientifique;

- elle doit être ouverte à toute communauté de recherche souhaitant l'utiliser, accessible sur la base de l'excellence scientifique évaluée par les pairs; elle doit donc disposer d'instances d'évaluation adéquates;

- elle peut conduire une recherche propre, et/ou fournir des services à une lou plusieurs) communauté(s) d'utilisateurs intégrant les acteurs du secteur économique. Ces communautés peuvent être présentes sur le site, venir ponctuellement y réaliser des travaux, ou interagir à distance. 
Par ailleurs, les infrastructures de recherche devront à l'avenir être en mesure de :

- produire une programmation budgétaire pluriannuelle ainsi qu'un budget consolidé intégrant les coûts complets;

- mettre à disposition les données produites, soit immédiatement, soit après une période d'embargo correspondant aux pratiques internationales du domaine concerné.

\section{QUATRE TYPES D"INFRASTRUCTURES}

La présente feuille de route française a été construite autour de quatre catégories d'infrastructures de recherche, selon leur caractère national ou multinational, leur mode de gouvernance et leur soutien budgétaire. Les critères précisés ci-dessus s'appliquent aux quatre catégories, qui sont définies sur les principes suivants :

- les Organisations Internationales (OI) sont fondées juridiquement sur une convention intergouvernementale parfois doublée d'un accord inter-agences et de statuts décrivant de façon détaillée la mise en œuvre de la convention ou des accords. La convention intergouvernementale, qui est assortie d'un protocole financier, précise notamment les objectifs de l'organisation, les conditions d'adhésion, les organes de fonctionnement, et les modalités de contribution des Etats membres;

- les Très Grandes Infrastructures de Recherche (TGIR) relèvent d'une stratégie gouvernementale traduite au moyen de plusieurs actions de la LOLF et d'un fléchage budgétaire du MENESR. Elles sont nationales ou font l'objet de partenariats internationaux ou européens, notamment par leur engagement dans la feuille de route du forum stratégique européen (ESFRI). Elles sont des instruments majeurs dans les réseaux de collaboration industrielle et d'innovation. Les TGIR sont sous la responsabilité scientifique des opérateurs de recherche; - les Infrastructures de Recherche (IR), relèvent des choix des différents opérateurs de recherche et sont mises en œuvre par eux, qu'il s'agisse des Alliances ou de leurs membres, ou d'établissements publics en raison de leurs missions particulières;

- les projets, en cours de construction ou déjà productifs mais n'ayant pas encore une pleine maturité selon les critères rappelés plus haut, ont déjà une existence et une importance dans le paysage de la recherche français qui justifient l'inscription sur la feuille de route. Ce statut pour une infrastructure a un caractère transitoire et fera l'objet d'une nouvelle analyse lors de la prochaine mise à jour de la feuille de route. 
Ces 4 types ne traduisent pas une hiérarchie d'excellence ou de nature technologique. Mis à part pour les $\mathrm{Ol}$, les structures juridiques ou légales, la dimension budgétaire ou encore les regroupements thématiques peuvent prendre des formes variées et ne sont donc pas des critères restrictifs distinguant les TGIR des IR ou des projets.

On peut remarquer que selon les critères présentés ci-dessus, les infrastructures d'essais et les démonstrateurs utilisés dans le cadre de grands programmes d'équipement (énergie, transports, bâtiment, agro-alimentaire, spatial, nucléaire, défense, etc) qui peuvent mener des recherches mais sans ouverture extérieure, n'ont pas vocation à être intégrées à la présente feuille de route.

\section{INFRASTRUCTURES DE RECHERCHE ET DONNÉES}

La production, le stockage et la mise à disposition de données sont des paramètres essentiels de la recherche d'aujourd'hui ; c'est particulièrement vrai pour les infrastructures de recherche. Certaines sont focalisées sur le numérique, en tant qu'objet des recherches elles-mêmes ou pour en développer les outils de calcul intensif, de transmission ou de stockage des données. D'autres ont pour but de mettre à disposition des bases de données, qu'elles soient brutes ou enrichies. Dans tous les cas, elles tendent à devenir découvrables, utilisables et interopérables par une communauté toujours plus large. Dans certains domaines, cette mise à disposition est immédiate et entièrement publique, suivant une directive européenne. Dans d'autres, une période d'embargo est la pratique courante avant dissémination. 


\section{LES INFRASTRUCTURES \\ DE RECHERCHE \\ ET LA FEUILLE DE ROUTE}

\section{L'INSCRIPTION SUR LA FEUILLE DE ROUTE}

La feuille de route nationale est un outil de pilotage stratégique du gouvernement qui est remis à jour tous les quatre ans selon un processus impliquant les alliances, organismes ou établissements tutelles, à l'issue duquel l'inscription peut être recommandée comme infrastructure ou comme projet. Ce processus est décrit en annexe de ce document. L'inscription témoigne de la volonté de l'Etat de structurer le paysage des infrastructures d'envergure nationale. Elle représente un label de qualité et une reconnaissance de sa valeur dans la Stratégie Nationale de Recherche (SNR) mais ne se substitue pas aux négociations entre partenaires : communautés scientifiques, collectivités territoriales, établissements publics, Etat et leurs équivalents à l'étranger.

Pour la première fois, la feuille de route nationale présente toute une variété d'informations précises telles que la dimension internationale, la localisation, le volume et la gestion des données ou encore les principales informations budgétaires. Si le résultat reste largement perfectible, il trace une nouvelle voie de suivi des infrastructures de recherche dont les informations pourront être régulièrement actualisées via la nouvelle mise en ligne de leurs fiches.

\section{FEUILLES DE ROUTE NATIONALE ET EUROPÉENNE}

La majorité des états membres de l'Union Européenne se livrent au même exercice stratégique : ec.europa.eu/research/infrastructures/index_en.cfm?pg= esfri-national-roadmaps.

Tous ont inscrit dans leur feuille de route nationale leur contribution à la feuille de route stratégique européenne (roadmap ESFRI pour European Strategic Forum for Research Infrastructures). Sa toute récente version de 2016 (www.esfri.eu/ esfri roadmap2016) présente trois catégories : 4 "emerging", 21 "projects " et 29 "landmarks", selon leur degré de maturité. Les nombreuses participations françaises à ces infrastructures sont mentionnées dans le présent document. 


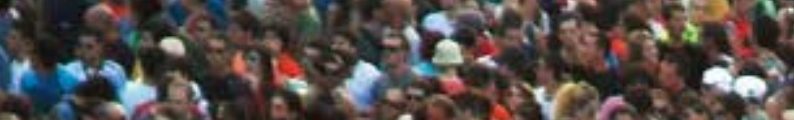

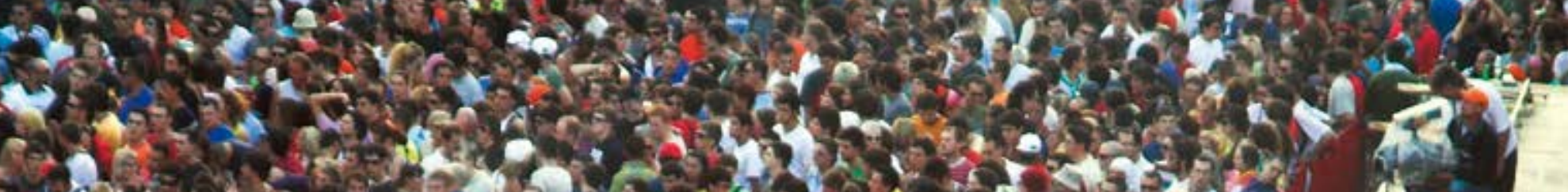

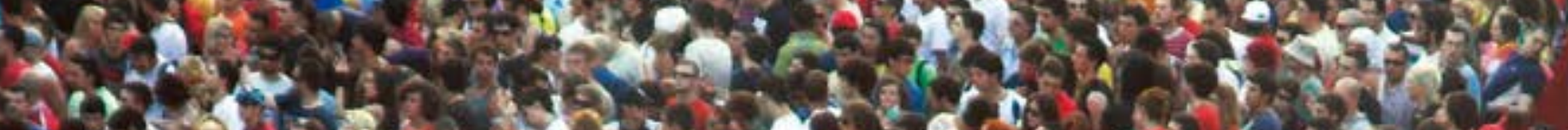

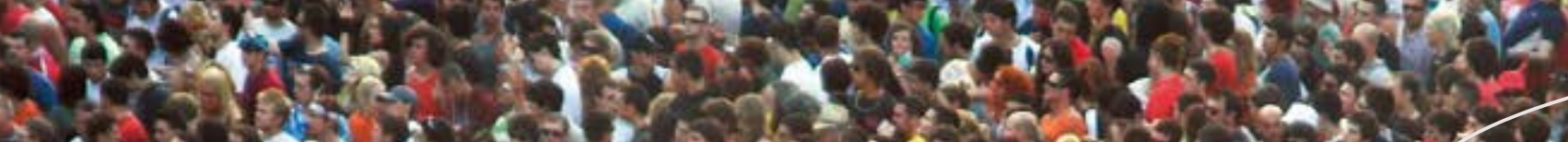

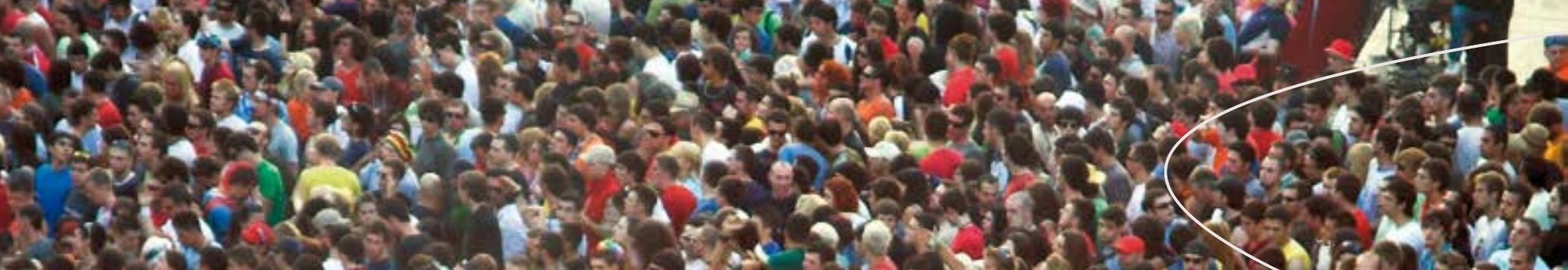

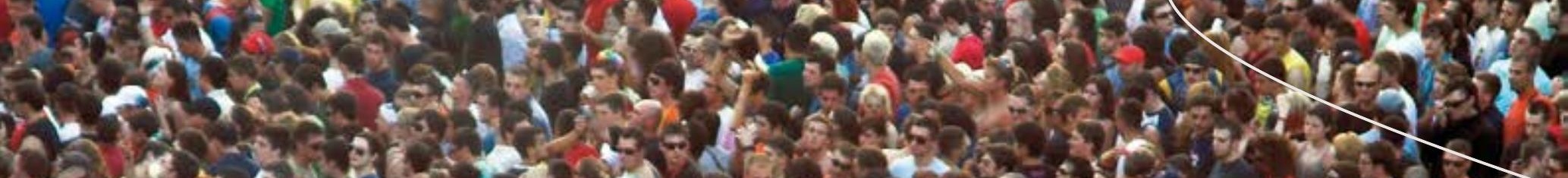

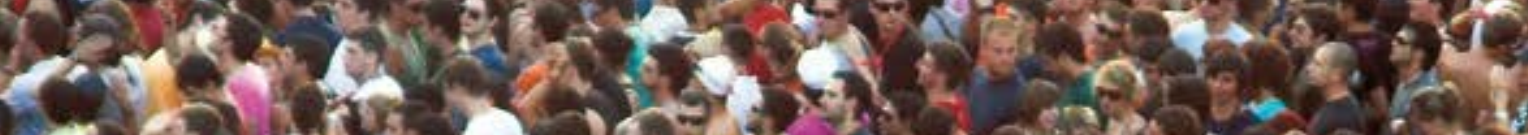

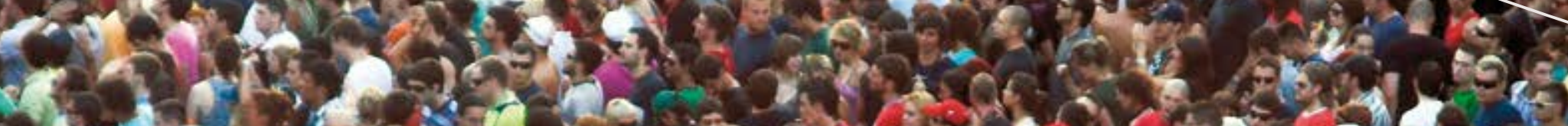

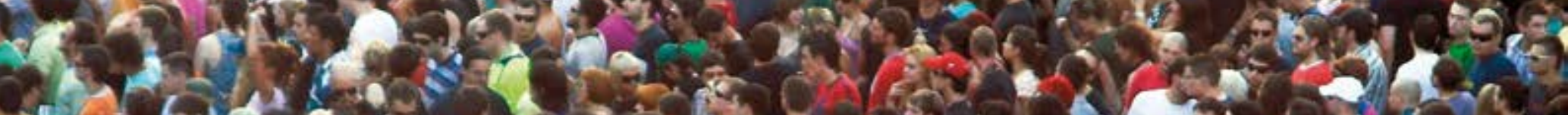

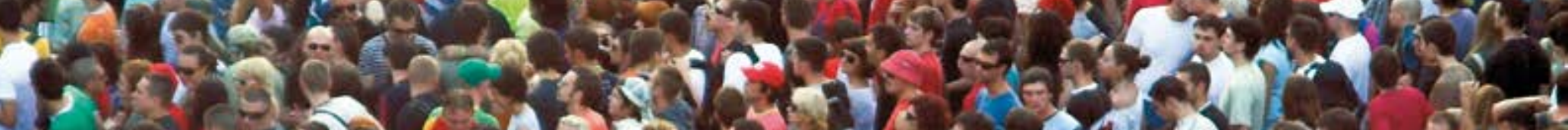

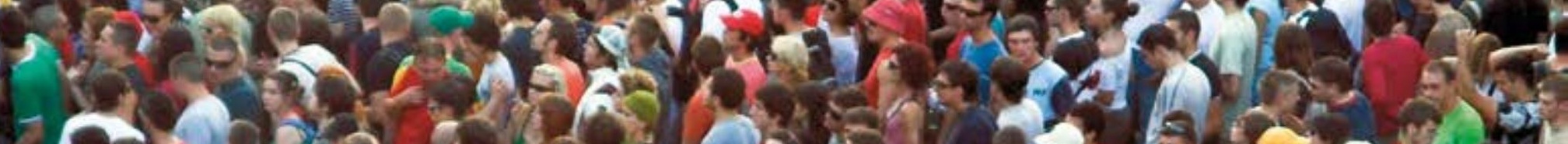

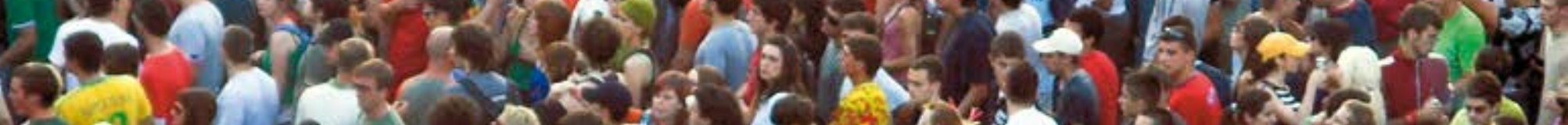
3
3 (5)

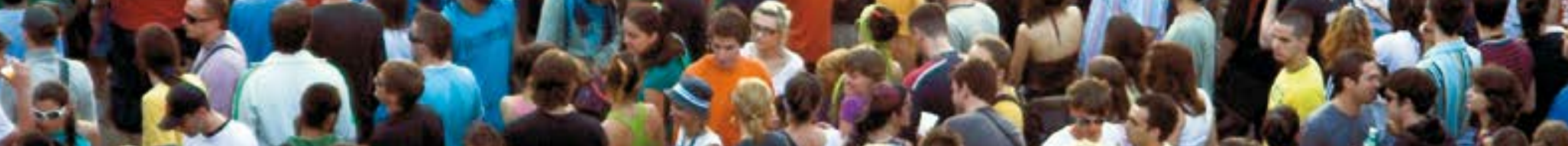

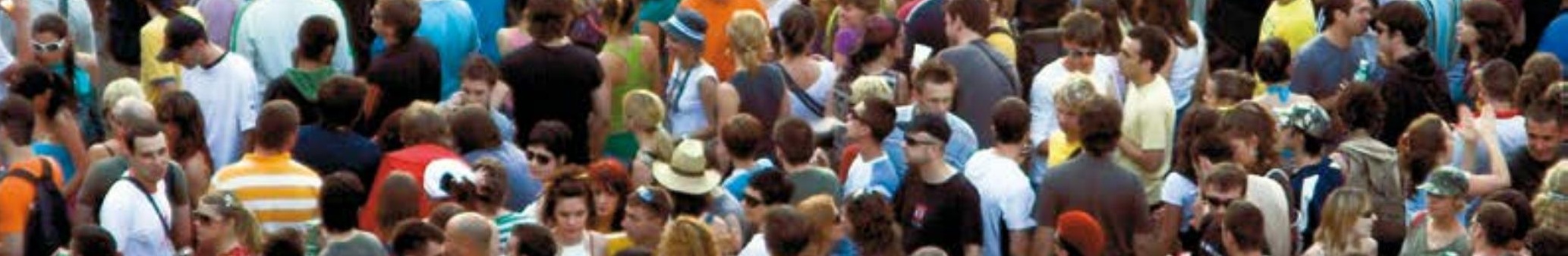

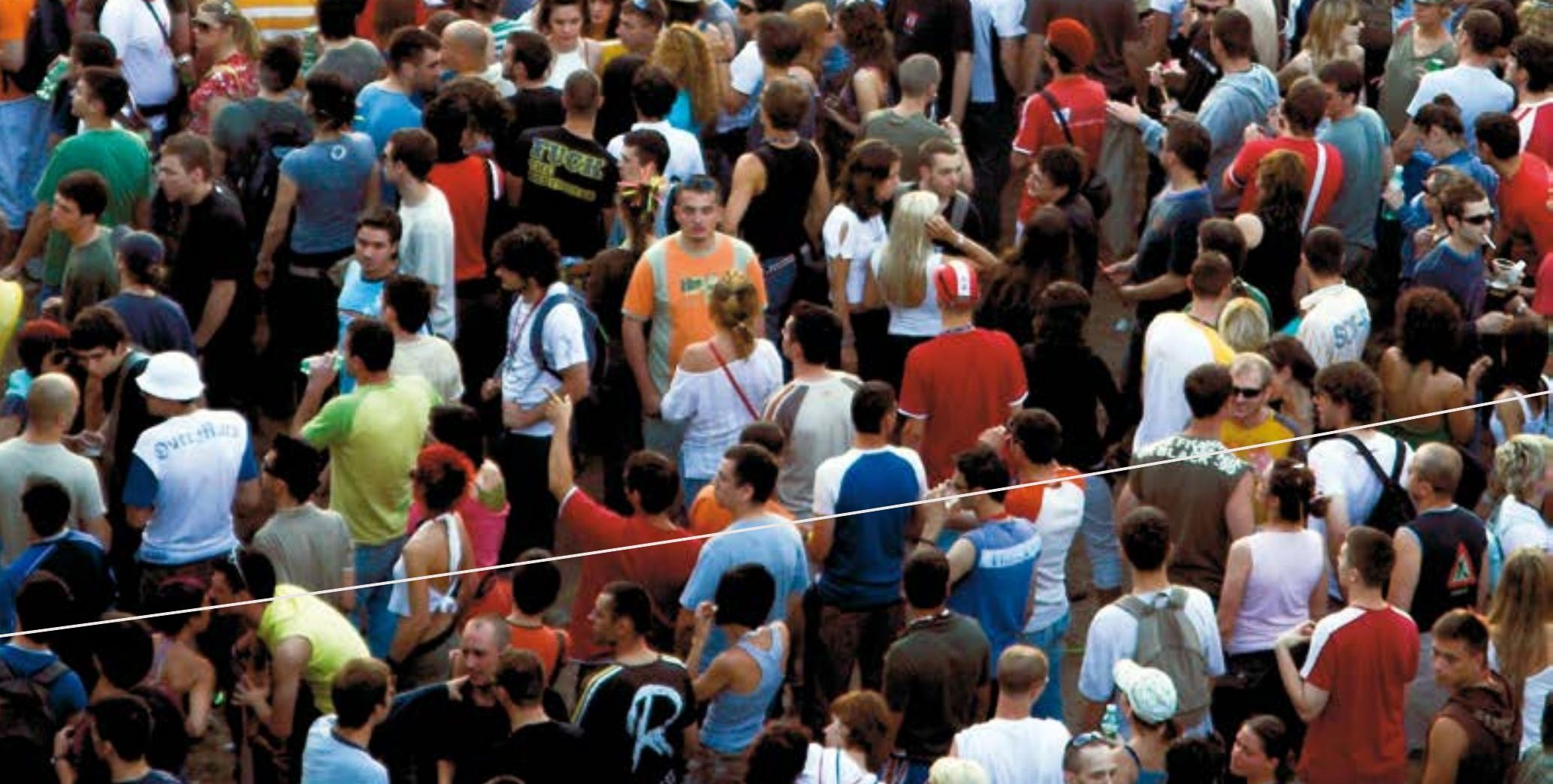


SCIENCES HUMAINES

ET SOCIALES

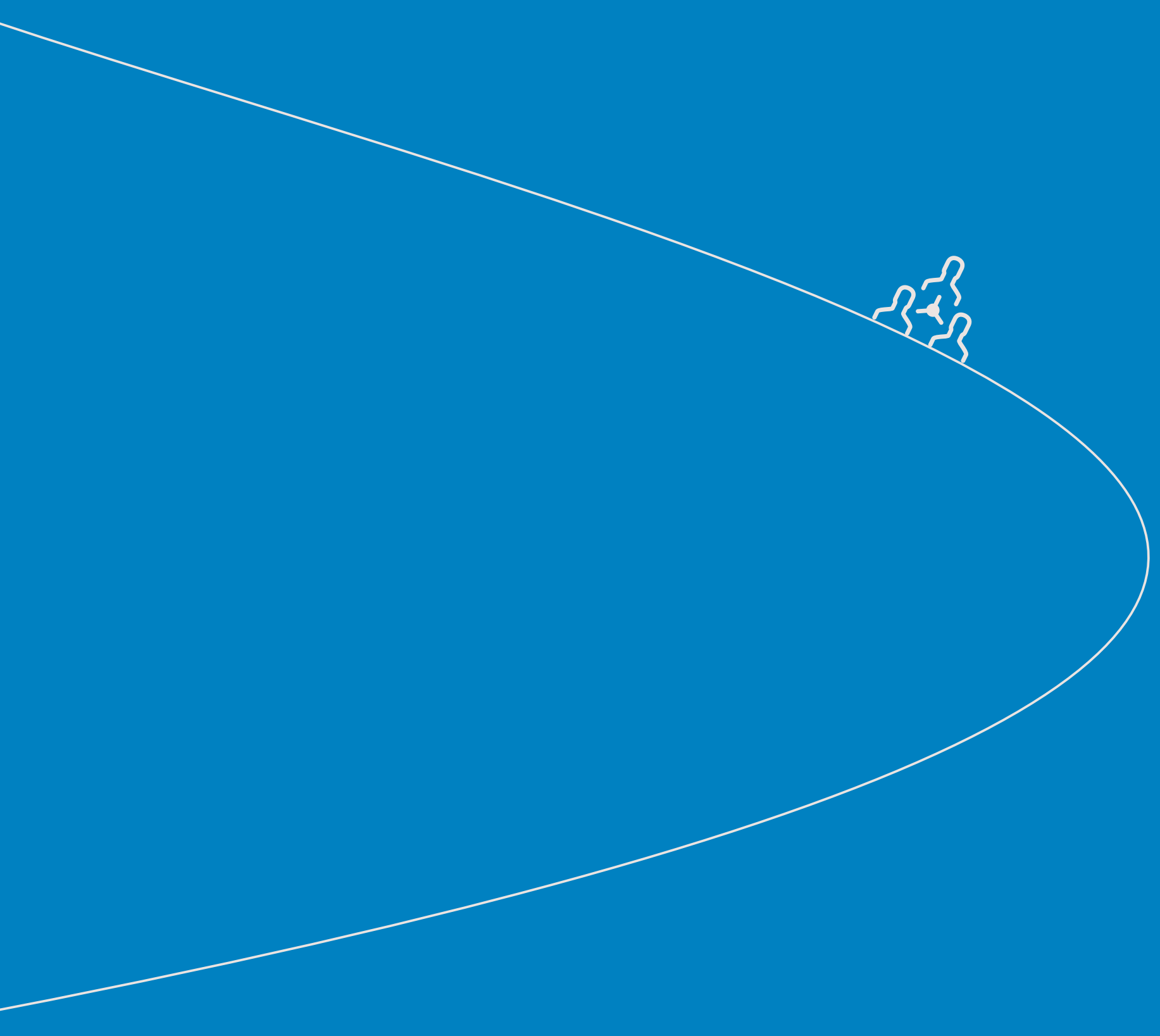




\section{Sciences Humaines}

\section{et Sociales}

Le développement du numérique combiné à celui de l'internet a donné accès à des données massives et aux moyens de calculs qui autorisent leur traitement, ainsi qu'à de nouveaux modes d'analyse de sources non numériques. Les disciplines des sciences humaines et sociales (SHS) se trouvent ainsi confrontées à une dynamique qui transforme le métier même du chercheur. Ainsi les infrastructures de recherche en SHS doivent permettre de constituer et de manipuler des corpus volumineux et très hétérogènes, de nature qualitative ou quantitative, susceptibles d'ouvrir de nouvelles voies de recherche et de favoriser l'interdisciplinarité. Inscrites dans un espace social largement ouvert au monde, les infrastructures contribuent à une meilleure valorisation d'un patrimoine scientifique et culturel et intéressent tous les établissements regroupés au sein de l'Alliance Athéna.

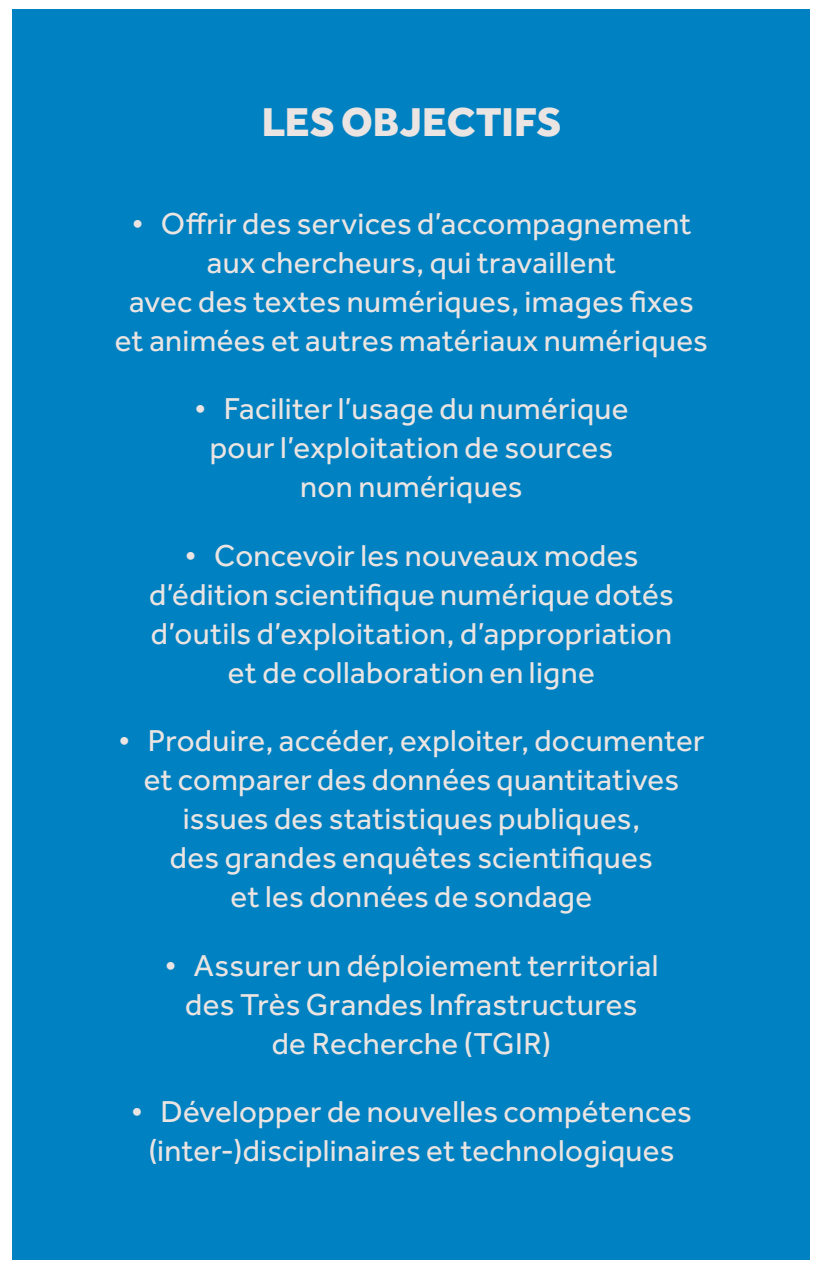

\section{1 \\ LES HUMANITÉS \\ NUMÉRIQUES}

-

Les facilités de stockage, et l'interactivité inhérente au numérique combinée à la diffusion de l'accès au réseau du World Wide Web ont ouvert des opportunités renouvelées en termes d'appropriation et de manipulation des ressources de recherche. En conséquence, on a assisté à une diversification des lieux de productions de ressources numériques qui ont mené à la création de nombreuses plateformes dédiées aux humanités numériques. Elles constituent des pôles de rassemblement de compétences disciplinaires et technologiques qui offrent de nombreux services pour accompagner les chercheurs en sciences humaines qui utilisent le numérique soit directement parce que les données de recherche sont numériques, soit comme environnement permettant l'accès à de nouveaux outils de traitement. Ces plateformes, souvent localisées dans les Maisons des Sciences de l'Homme, se développent en coordination très étroite avec la TGIR HUMA-NUM. La TGIR met à leur disposition, ainsi qu'à l'ensemble des laboratoires en SHS de l'enseignement supérieur et de la recherche, des outils et services de stockage, de traitement, d'interopérabilité, de diffusion, d'exposition, de signalisation et d'archivage de données numériques dédiées aux SHS en y incluant les aspects de sédimentation des connaissances et les besoins spécifiques en matière d'accès à ces dernières. Au niveau européen, HUMA-NUM coordonne la participation de la France dans I'infrastructure européenne DARIAH et prépare la participation à CLARIN.

\section{2}

\section{EDITION NUMÉRIQUE POUR LES SHS}

L'infrastructure OpenEdition conçoit les nouveaux modes d'édition scientifique numérique dotés d'outils d'exploitation, d'appropriation et de collaboration en ligne. L'infrastructure permet d'améliorer l'impact des projets de recherche pluridisciplinaires et leur capacité à transférer leurs résultats aux acteurs socio-économiques pour répondre aux défis sociétaux aux niveaux national et 
européen. OpenEdition est un acteur leader aux niveaux européen et international pour ce qui concerne l'innovation dans le domaine de l'édition scientifique en libre accès. Cette infrastructure participe à l'amélioration de la qualité des recherches par une meilleure circulation des savoirs scientifiques et par une amélioration des conditions de son évaluation. II s'agit de garantir à la fois la transparence, la qualité scientifique et éditoriale ainsi que la durabilité.

Openedition est aussi listée dans le domaine « Information scientifique et technique " pour signaler son double caractère en tant qu'infrastructure de recherche pour les SHS et pour l'édition numérique.

\section{3}

\section{LES INFRASTRUCTURES DE DONNÉES}

Les statistiques publiques, les grandes enquêtes scientifiques, les données de gestion ou les données de sondage représentent une source de connaissance essentielle pour les sciences sociales. La construction d'indicateurs européens sur la société via les enquêtes longitudinales européennes est un enjeu qui participe à la construction de l'Europe. La TGIR PROGEDO a pour vocation d'organiser, au niveau français, les services de production et gestion des données d'enquêtes pour la recherche en SHS et de développer la culture des données dans les universités; ceci pour donner à la France la possibilité de prendre une part active à la construction de ces indicateurs sur la société européenne. Un autre enjeu de cette TGIR est de permettre aux chercheurs d'ancrer leur recherche sur des données des grandes enquêtes ainsi produites.

L'infrastructure repose à la fois sur les Plate-formes Universitaires de Données (PUD) basées dans les Maisons des Sciences de l'Homme (MSH) et sur la tête de réseau de diffusion des données CESSDA FR - Réseau Quetelet. Elle est construite autour de quatre départements correspondant aux consortiums européens CESSDA (Council of European Social Sciences Data Archives), ESS (European Social Survey), SHARE (Survey of Health, Ageing and Retirement in Europe) et GGP (Generation and Gender Program).

\section{4}

\section{UNE INFRASTRUCTURE D'INTERFACE TERRITORIALE}

Le Réseau national des Maisons des Sciences de l'Homme (RnMSH) et ses 23 maisons sont des instruments de transformation des sciences humaines et sociales basés sur l'interdisciplinarité et le développement de la logique de projets. Le Réseau joue un rôle majeur dans le développement et le déployement de l'interdisciplinarité et dans la structuration de l'ingénierie qui lui est associée. Les MSH sont des lieux de partenariat fort entre les universités, les organismes (en particulier le CNRS) et les collectivités territoriales.

En matière d'infrastructures, deux grandes missions des MSH sont essentielles :

- Relayer pour déployer et ancrer territorialement les dispositifs nationaux que sont notamment les deux TGIR en SHS et le Consortium de Valorisation Thématique (CVT) SHS. Parce que ces dispositifs nationaux sont souvent coordonnés à des dispositifs européens, les MSH doivent ainsi faciliter l'accès des communautés scientifiques aux dynamiques européennes sur les infrastructures de recherche (forum européen ESFRI, et volet «infrastructures » du programme Horizon 2020).

- Accélérer les transformations nécessaires des SHS à la recherche sur projets avec l'approche par Défis Sociétaux. Ainsi, les MSH facilitent l'incubation de recherches à forte innovation et prise de risque, qu'elles soient internes au champ des SHS ou conduites avec d'autres secteurs disciplinaires.

\section{5}

\section{UNE INFRASTRUCTURE TRANSVERSE POUR LES SCIENCES DU PATRIMOINE}

Au cours des années récentes les sciences du patrimoine ont connu un essor considérable en Europe. Le projet d'infrastructure européenne ERIHS répond à la nécessité d'établir une structure robuste pour organiser le domaine de recherche et renforcer la position internationale de l'Europe dans ce champ éminemment interdisciplinaire.

En outre, on attend d'ERIHS une consolidation de l'articulation, déjà bien établie dans ce domaine de recherche, entre les méthodes issues de la physique, de la chimie, de l'anthropologie biologique, de la géologie et des sciences de l'information d'une part, et la méthodologie traditionnelle des disciplines interprétatives (histoire, histoire de l'art, archéologie, etc).

Ce caractère transversal d'E-RIHS est mis en avant en intégrant également l'infrastructure dans le domaine "Sciences analytiques". E-RIHS est une des nouvelles infrastructures de recherche incluses dans la feuille de route européenne ESFRI 2016. 
LISTE DES INFRASTRUCTURES DE RECHERCHE

DANS LE DOMAINE SCIENCES HUMAINES ET SOCIALES

\begin{tabular}{|c|c|c|c|}
\hline CATÉGORIE & NOM & NOM COMPLET & ESFRI \\
\hline TGIR & Huma-Num & Humanités Numériques & DARIAH (2006) \\
\hline TGIR & Progedo & PROduction et GEstion de DOnnées & $\begin{array}{l}\text { ESS }(2006) \\
\text { CESSDA (2006) } \\
\text { SHARE (2006) } \\
\text { GGP (2016) }\end{array}$ \\
\hline IR & OpenEdition ${ }^{1}$ & $\begin{array}{l}\text { Edition électronique ouverte en Sciences } \\
\text { humaines et sociales }\end{array}$ & \\
\hline IR & $\mathrm{RnMSH}$ & Réseau national Maison des Sciences de l'Homme & \\
\hline Projet & $E R I H S-F R^{2}$ & $\begin{array}{l}\text { European Research Infrastructure for Heritage } \\
\text { Science }\end{array}$ & ERIHS (2016) \\
\hline
\end{tabular}

1 Relève également du secteur «Information Scientifique et Technique», dans lequel la fiche de l'infrastructure est présentée.

2 Relève également du secteur «Sciences de la Matière et Ingénierie ». 
Huma-Num est une très grande infrastructure (TGIR) visant à faciliter le tournant numérique de la recherche en sciences humaines et sociales.

Pour remplir cette mission, la TGIR Huma-Num est bâtie sur une organisation originale consistant à mettre en œuvre un dispositif humain (concertation collective) et technologique (services numériques pérennes) à l'échelle nationale et européenne en s'appuyant sur un important réseau de partenaires et d'opérateurs.

La TGIR Huma-Num favorise ainsi, par l'intermédiaire de consortiums regroupant des acteurs des communautés scientifiques, la coordination de la production raisonnée et collective de corpus de sources (recommandations scientifiques, bonnes pratiques technologiques). Elle développe également un dispositif technologique unique permettant le traitement, la conservation, l'accès et l'interopérabilité des données de la recherche. Ce dispositif est composé d'une grille de services dédiés, d'une plateforme d'accès unifié (ISIDORE) et d'une procédure d'archivage à long terme.

La TGIR Huma-Num propose en outre des guides de bonnes pratiques technologiques généralistes à destination des chercheurs. Elle mène régulièrement des actions d'expertise et de formation.

Elle porte la participation de la France dans le projet DARIAH en coordonnant les contributions nationales.

\section{IMPLICATIONS SOCIO-ÉCONOMIQUES}

La TGIR travaille avec le monde de l'industrie de la connaissance (SPARNA, Limonade \& co, ActiveCircle, Mondéca...), du search engine et du big data (Antidot) dans le but d'améliorer l'appropriation, par les communautés SHS (dont les MSH), des enjeux de l'économie de la donnée numérique.

\section{DONNÉES}

Estimation du flux de données : Entre 1 Po et 2 Po

Stockage : Les données sont stockées dans l'infrastructure Huma-Num hébergée au Centre de Calcul de I'IN2P3-CNRS et archivées au CINES.

Accessibilité : Les données sont en accès libre.

Présence dans des réseaux de données : ISIDORE et NAKALA, DARIAH, PARTHENOS, Humanites at Scale, RnMSH et Openedition.

\section{Coût de construction \\ -}

$1,4 \mathrm{M} €$ (hors personnel)

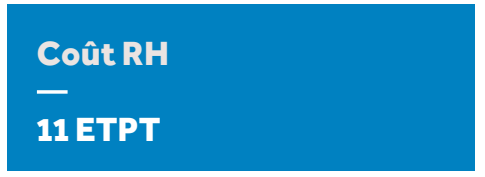

\section{Dimension internationale}

DARIAH-ERIC (Digital Research Infrastructure for the Arts and the Humanities), ESFRI Landmark www.dariah.eu

CLARIN-ERIC (Common Language Ressources and Technology Infrastructure), ESFRI Landmark www.clarin.eu

Coordinateur : DARIAH-ERIC : FR, Humanum, CLARIN ERIC : NL

Pays partenaires : DARIAH-ERIC : AT, DE, BE, CY, HR, DK, FR, GR, IR, IT, LU, MT, NL, SR, SI, CLARIN-ERIC: AT, BG, CZ, DK, Dutch Language Union $\mathrm{EE}, \mathrm{FI}, \mathrm{DE}, \mathrm{GR}, \mathrm{IT}, \mathrm{LT}, \mathrm{NL}, \mathrm{NO}, \mathrm{PL}, \mathrm{PT}, \mathrm{SI}, \mathrm{SE}, \mathrm{UK}$ (la participation de FR est en préparation)

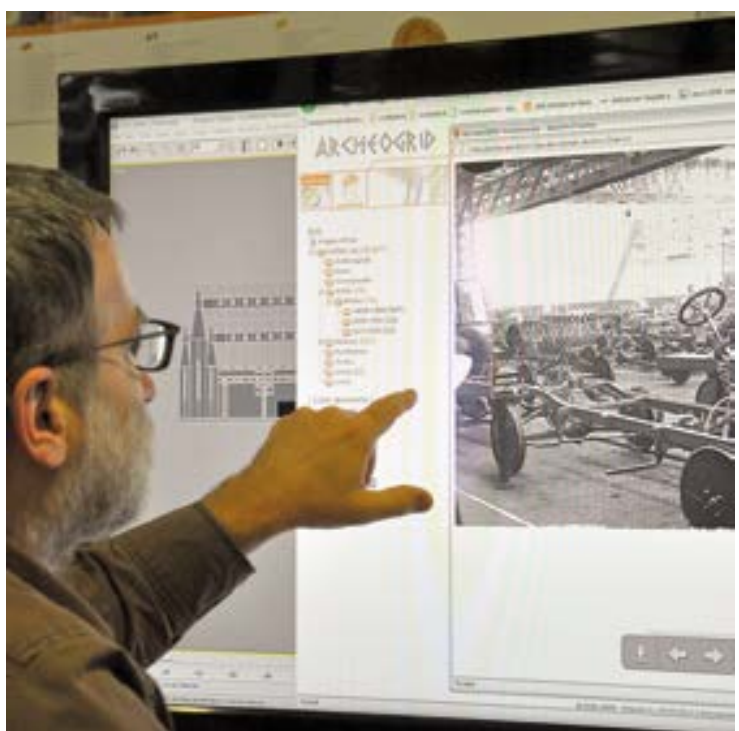

Type de l'infrastructure : TGIR

Statut de l'infrastructure :

Unité mixte de service

Localisation:

Paris

Localisation des autres sites :

Lyon

Responsables de l'infrastructure :

Olivier BAUDE, Stéphane POUYLLAU

Création :

Exploitation:

2013

Tutelles / Partenaires :

CNRS, AMU, Campus Condorcet

\section{Contact en France :}

direction@huma-num.fr

www.huma-num.fr 


\section{PROduction et GEstion de DOnnées}

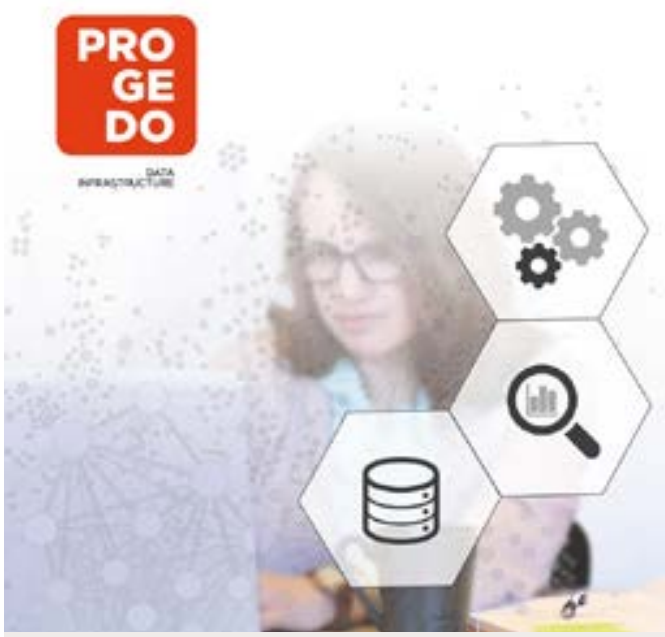

Type de l'infrastructure : TGIR

Statut juridique de l'infrastructure :

Unité mixte de service

\section{Localisation :}

Paris

\section{Localisation des autres sites :}

Caen, Dijon-Besançon, Lille, Lyon, Nantes,

Strasbourg, Toulouse

\section{Responsable de l'infrastructure:}

Pascal BULEON

\begin{tabular}{|c|c|c|}
\hline Création : & Exploitation : & Maintenance: \\
\hline 2014 & 2014 & 2016 \\
\hline
\end{tabular}

Tutelles / Partenaires :

CNRS, EHESS, FNSP, INED, GENES,

Univ. Paris-Dauphine

\section{Contact en France :}

info@progedo.fr

\section{www.progedo.fr}

PROGEDO est l'acteur central des politiques ministérielles s'agissant de la production et de la gestion de données en sciences humaines et sociales (SHS). L'infrastructure a pour mission de développer la culture des données et de hausser le niveau de structuration nationale des communautés de recherche en déployant une stratégie de développement entre les organismes de recherche, les grands établissements et les universités, et de renforcer la position de la France dans l'espace européen de la recherche.

Pour ce faire, PROGEDO organise l'appui à la collecte, à la documentation, à la préservation et à la diffusion d'un vaste ensemble de données nécessaires à la recherche en SHS et participe à la mise en place des dispositifs sécurisés d'accès aux micro-données. PROGEDO soutient également la réalisation de grandes enquêtes internationales et offre un accès à certaines bases de données étrangères en SHS.

Implantée dans les universités sur tout le territoire grâce à ses platesformes universitaires de données, PROGEDO constitue localement un atout de formation et de recherche ainsi qu'une porte d'entrée sur les infrastructures européennes et les dispositifs internationaux.

PROGEDO contribue également, en concertation avec les Alliances ATHENA et AVIESAN, à l'articulation de travaux pluridisciplinaires entre grands domaines conjointement concernés par les sciences humaines et sociales.

\section{IMPLICATIONS SOCIO-ÉCONOMIQUES}

Les données des grandes enquêtes européennes sur les conditions de vie des seniors, le noyau familial, l'éducation, ou encore les valeurs sont exploitables pour la prise de décisions et la définition des politiques publiques.

Stockage : Les données issues des grandes enquêtes européennes (ESS, SHARE, GGP) sont centralisées sur des sites européens uniques. Celles de CESSDA France sont sur les serveurs des unités partenaires du réseau Quetelet.

Accessibilité : Les données européennes ESS, SHARE et GGP sont en accès libre pour la communauté de recherche après enregistrement. Celles des statistiques publiques sont accessibles selon deux niveaux d'accès et de sécurisation. D'autres données d'enquêtes académiques, agrégées et documentées, sont accessibles dans une finalité de recherche auprès de l'INED et du CDSP.

\section{Coût de fonctionnement \\ 4,249 M€ (hors personnel)}

Coût RH

65,23 ETPT

\section{Dimension internationale}

CESSDA AS (Consortium of European Social Science Data Archives), ESFRI Landmark

ESS ERIC (European Social Survey), ESFRI Landmark

SHARE ERIC (Survey of Health, Ageing and Retirement), ESFRI Landmark

Coordinateur : CESSDA AS : NO, ERIC ESS : UK, SHARE ERIC : DE

Site internet : www.cessda.net

www.europeansocialsurvey.org

www.share-project.org 


\section{Réseau national des Maisons des Sciences de l'Homme}

Le Réseau national des Maisons des Sciences de l'Homme organise au plan national les actions portées par les $23 \mathrm{MSH}$. Ceci concerne :

1. la construction de plates-formes de grande ampleur, fonctionnant sur le principe de la complémentarité des ressources, des équipements et des personnels:

2. I'incitation à l'incubation de projets résolument pluridisciplinaires (au sein des SHS ou hors SHS);

3. la mise en œuvre d'opération de valorisation de recherches;

4. I'ancrage des actions européennes et internationales dans les MSH, et des activités portées par les TGIR Huma-Num et PROGEDO.

L'objectif premier en termes d'infrastructure est de mettre en place cinq plates-formes d'ampleur qui regroupent de façon variable les $23 \mathrm{MSH}$ :

1. Plate-forme Spatio (données spatialisées):

2. Plate-forme Scripto (données notées):

3. Plate-forme Visio (corpus audio-visuels)

4. Plate-forme Cogito (plate-forme d'équipement pour les recherches en sciences cognitives et données issues des expérimentations sur ces équipements ou d'autres):

5. Plate-forme Data (corpus de données quantitative).

et d'inciter à l'utilisation d'une 6e plate-forme d'internationalisation

6. plate-forme Fundit.fr (portail internet consolidant les appels d'offres relatifs à la mobilité et au financement de la recherche aux niveaux européens et internationaux dans le domaine des SHS - plate-forme développée par la fondation RFIEA en partenariat avec la FMSH et avec le soutien du CNRS).

\section{IMPLICATIONS SOCIO-ÉCONOMIQUES}

Le dernier appel à projet conjoint du CVT (Consortium de Valorisation Thématique) Athéna et du RnMSH a permis de soutenir des projets de valorisation à forte retombée économique dans le domaine de l'Intelligence sémantique, dans le traitement de documentations numériques complexes ou encore dans le développement de solution d'apprentissage lexical de langues étrangères.

\section{DONNÉES}

Accessibilité : Les données sont des données en accès libre pour les chercheurs (selon des modalités d'enregistrement variables), à l'exclusion de toute finalité commerciale.

Présence dans des réseaux de données : PUD (Plates-Formes Universitaires de Données) au sein des TGIR PROGEDO et Huma-Num, et de DARIAH.

\section{Coût de fonctionnement \\ 4,9 M€}

\section{Coût RH} 403 ETPT

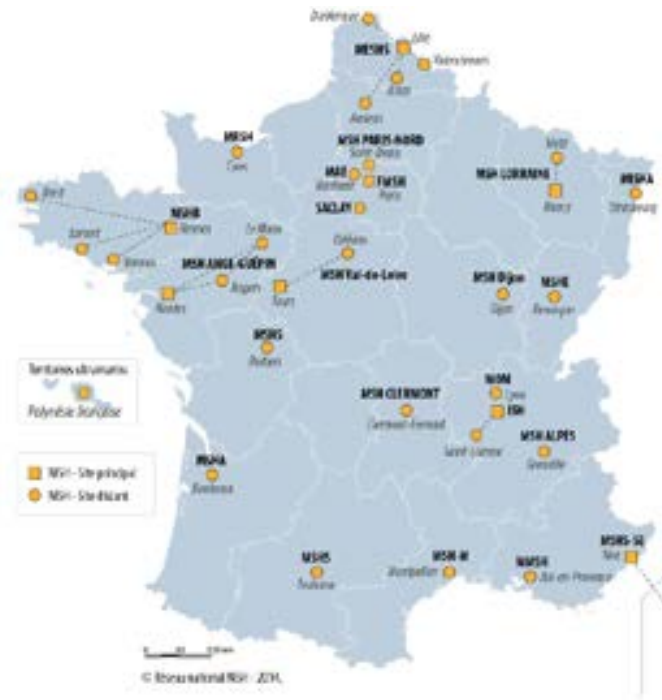

Type de l'infrastructure : IR

Statut de l'infrastructure :

Groupement d'Intérêt Scientifique

Localisation :

Paris

\section{Localisation des autres sites :}

Angers, Aix-en-Provence, Besançon,

Bordeaux, Brest, Caen, Clermont-Ferrand, Corte, Dijon, Grenoble, Le Mans, Lille, Lorient, Lyon, Nancy, Metz, Montpellier, Nanterre, Nantes, Nice, Orleans, Poitiers, Rennes, Saclay, Saint-Denis, Saint-Etienne, Strasbourg Toulouse, Tours, Vannes

\section{Responsable de l'infrastructure: Philippe VENDRIX}

\section{Création: Exploitation: \\ 2006

Tutelles / Partenaires:

CNRS, les universités (ou COMUE), FMSH

\section{Contact en France :}

vendrix@univ-tours.fr

www.msh-reseau.fr 


\section{European Research Infrastructure for Heritage Science}

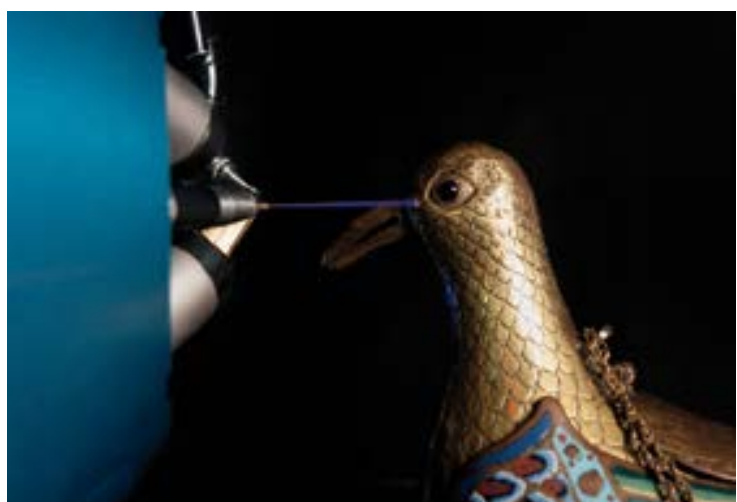

Type de l'infrastructure : Projet

\section{Localisation : \\ Paris}

Localisation des autres sites :

île-de-France

Responsables de l'infrastructure :

Isabelle PALLOT-FROSSARD, Loic BERTRAND

\begin{tabular}{|c|c|}
\hline Création: & Exploitation: \\
\hline prévue & prévue \\
en 2019 & en 2019 \\
\hline
\end{tabular}

\section{Tutelles / Partenaires :}

Les tutelles et partenaires impliqués au titre des fournisseurs d'accès sont : CNRS, FSP, INRIA, MCC, MNHN, UVSQ

\section{Coordinateur de l'infrastructure internationale :}

Etienne SNOECK (CNRS-CEMES)

\section{Contact en France :}

isabelle.pallot-frossard@culture.gouv.fr loic.bertrand@synchrotron-soleil.fr
E-RIHS répond aux problèmes expérimentaux que posent la connaissance et la conservation des matériaux du patrimoine (collections des musées et muséums, ensembles monumentaux, sites archéologiques, fonds d'archives, de bibliothèques, etc.), qui se caractérisent par une hétérogénéité multi-échelle et un vieillissement au temps long (peintures, céramiques et verres, métaux, spécimens paléontologiques, matériaux lithiques, documents graphiques, etc.).

E-RIHS développe un accès transnational à des dispositifs distribués dans nombre de pays d'Europe, regroupés en 4 plateformes :

- FIXLAB : accès à des équipements fixes permettant des analyses synchrotron, par faisceaux d'ions, lasers, etc.;

- MOLAB : accès à des équipements mobiles destinés à étudier les matériaux in situ:

- ARCHLAB : accès aux archives d'institutions en charge de la conservation de biens culturels (données scientifiques, photographies, notes, rapports de restauration, etc.);

- EXPERTLAB : constitution de panels d'experts pour l'initiation de projets intégrés d'étude de biens patrimoniaux.

E-RIHS intervient spécifiquement dans le cadre d'études complexes pour lesquelles le développement d'outils avancés d'analyse et d'imagerie de haute définition est nécessaire et qui ont un impact direct sur la conservation. Le domaine privilégié d'intervention de l'infrastructure est celui de l'étude de larges corpus, collections et ensembles architecturaux.

\section{IMPLICATIONSSOCIO-ÉCONOMIQUES}

Science des matériaux : vieillissement de matériaux.

Méthodologie instrumentale : en imagerie et spectroscopie, méthodes d'analyse non invasives et portables, couplages de méthodes analytiques.

Méthodes de conservation-restauration : procédés respectueux de l'environnement.

Sciences humaines et sociales : traitement et archivage des données numériques, réflexivité, épistémologie, interdisciplinarité.

\section{DONNÉES}

Stockage : Le Centre national de calcul intensif, IDRIS, est I'un des fournisseurs d'accès de l'infrastructure. Les différents volets afférents aux données seront mis en place dans le cadre de ce partenariat.

Accessibilité : Les données sont libres d'accès pour les chercheurs.

Présence dans des réseaux de données : DARIAH-ERIC. INRIA et IDRIS. Réseaux de collaboration : PARTHENOS, Labex PATRIMA.

\section{Dimension internationale}

E-RIHS, ESFRI Roadmap

Coordinateur : En discussion, projet d'infrastructure déposé par l'Italie sur la liste d'infrastructures ESFRI

Pays partenaires : $B E, C Y, C Z, D E, E S, F R, H E, H U, I T, N L, P T, U K$ 
SCIENCES HUMAINES ET SOCIALES 

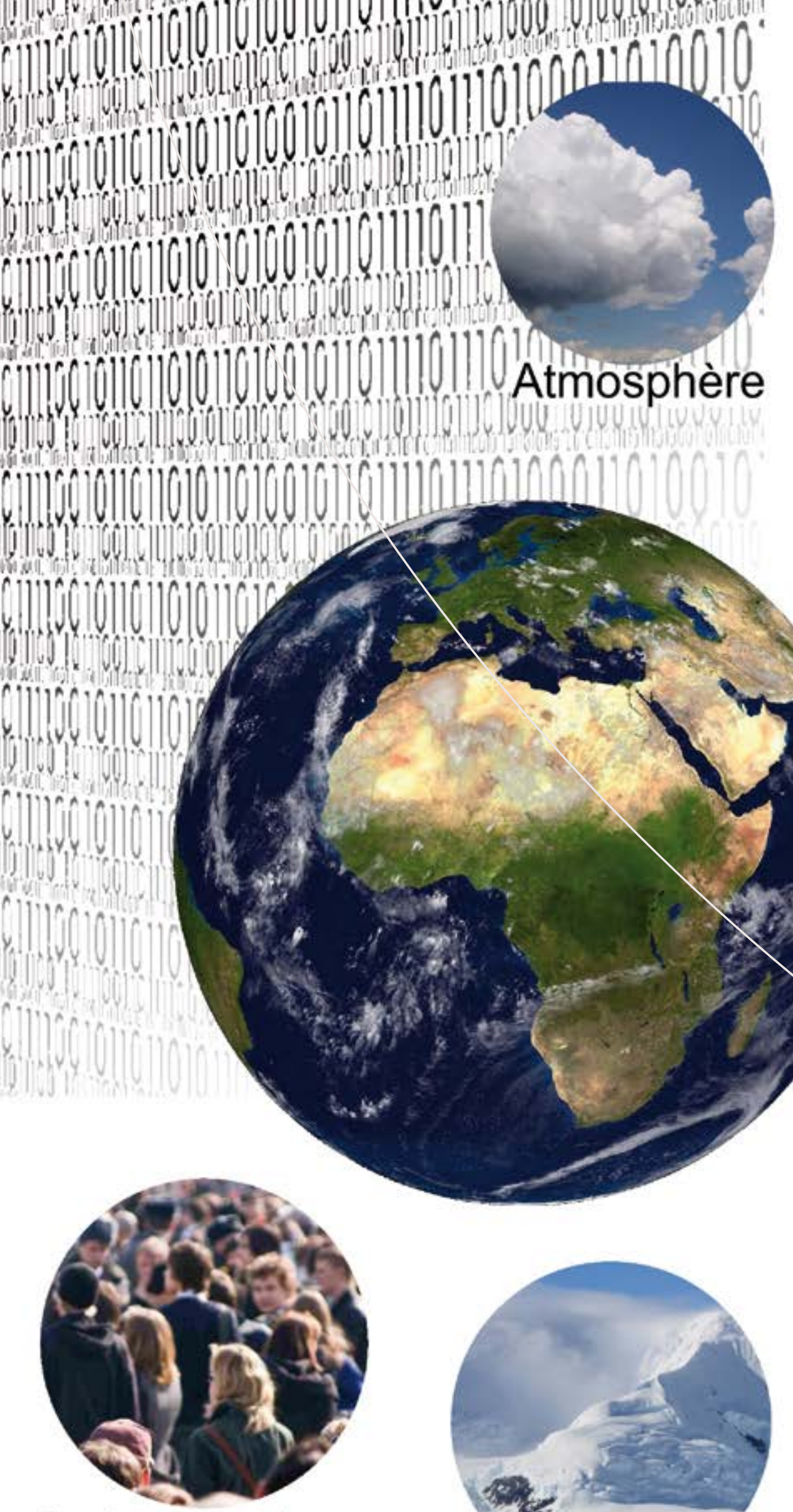

Anthroposphère

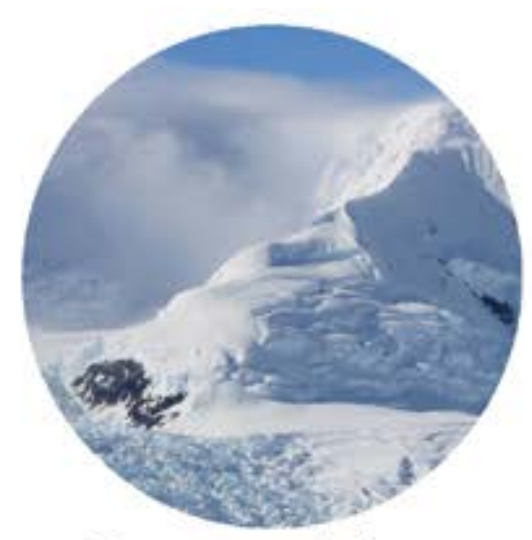

Cryosphère

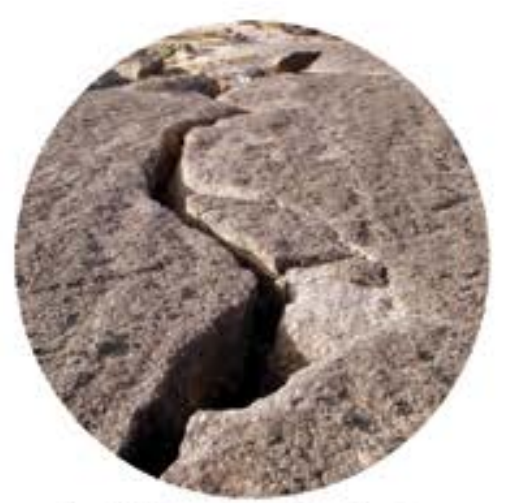

Lithosphère

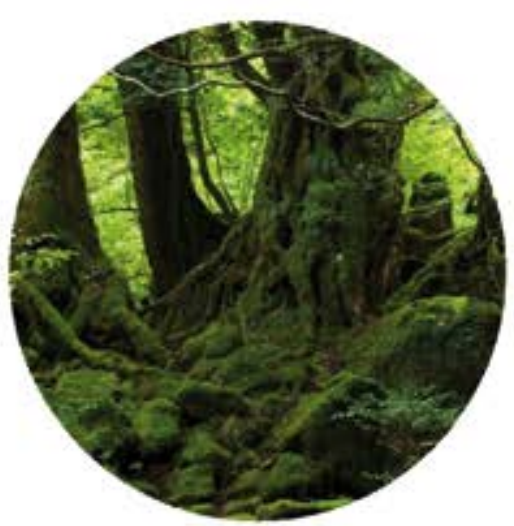

\section{Biosphère}

\section{Hydrosphère}




\section{Sciences du Système Terre et de l'Environnement}

\author{
Les infrastructures de recherche \\ du domaine Système Terre et \\ Environnement investiguent les fronts de \\ connaissance sur les processus à l'œuvre \\ au sein des grands compartiments \\ de la planète, et entre eux.
}

Les données de recherche délivrées par les infrastructures du domaine permettent de :

1. comprendre, modéliser et scénariser l'évolution du climat, de la biodiversité et des ressources;

2. suivre les pollutions et leurs impacts;

3. développer les recherches sur l'adaptation ou la mitigation;

4. aider la prise de décision face au risque et évaluer les effets des politiques publiques. Elles sont orientées vers la production de services et le développement économique dans les nouveaux secteurs offerts par les transitions énergétique et écologique.

Ces infrastructures, souvent distribuées, sont très diverses. Certaines dites "logistiques " permettent l'accès au terrain comme les flottes ou les stations. D'autres sont des réseaux de dispositifs d'observation, d'expérimentation, de collection et d'analyse. D'autres encore sont virtuelles et dédiées à l'accès et la mise à disposition de données scientifiquement validées ainsi que de produits à valeur ajoutée (modèles, cartes, simulations...).

Elles sont pensées à l'échelle européenne (feuille de route ESFRI, cluster ENVRIplus Environmental Research Infrastructures providing shared solutions for science and society) ou internationale. Elles sont bâties à partir de dispositifs labélisés par les établissements de recherche ou par l'Alliance AllEnvi pour les SOERE ${ }^{1}$. Soutenues par le MENESR, les établissements ou le CGI, elles sont multi-tutelles, leur gouvernance intégrant la diversité des établissements partenaires. AllEnvi assure la cohérence de l'ensemble face aux besoins des scientifiques, exprimés lors d'exercices de prospective récurrents.

Le domaine comporte aussi des organisations internationales (OI), porteuses de services aval dans les secteurs de l'environnement et de l'espace, comme l'ESA ou Eumetsat, ou bien des sociétés comme Mercator en lien avec le programme Copernicus. Seul le Centre Européen pour les

1 Systèmes d'Observation et d'Expérimentation de long terme pour la Recherche en Environnement.
Prévisions Météorologiques à Moyen Terme (CEPMMT) figure dans la présente feuille de route dès lors qu'il met à disposition des chercheurs, des données, des outils et de la formation. Au-delà, les sciences de l'environnement sont aussi utilisatrices de grands équipements analytiques (Soleil, ESRF...) et numériques (Genci, Renater...).

\section{INFRASTRUCTURES " LOGISTIQUES "}

Les infrastructures "logistiques" sont incontournables pour l'ensemble de la communauté nationale, et souvent internationale. Elles permettent l'acquisition de données in situ et la collecte d'échantillons. II s'agit des flottes (FOF pour les bateaux, SAFIRE pour les avions), de la contribution au consortium européen des navires foreurs (IODP/ ECORD) ou de stations comme CONCORDIA en Antarctique. On peut également citer, hors de la feuille de route, des dispositifs nationaux comme la flotte "Ballons " opérée par le CNES ou la station arctique du Svalbard co-gérée avec l'Allemagne par I'Institut Polaire Français IPEV.

\section{TERRE INTERNE}

-

RESIF/EPOS est une infrastructure d'observation qui réunit l'ensemble des moyens géophysiques nationaux dédiés au suivi des aléas et des ressources de la Terre interne.Très intégrative, elle représente un modèle de structuration de systèmes d'observation opérés par plusieurs établissements. Elle a pour ambition de s'ouvrir à d'autres types de données (géochimie,...). Le pôle Terre Interne (FORM@TER) du PÔLE DE DONNÉES complète le dispositif et constitue avec une partie de RESIF le miroir de I'ESFRI EPOS.

\section{ATMOSPHÈRE}

Les infrastructures d'observation sur l'atmosphère sont des miroirs d'ESFRI. Elles sont portées par des communautés structurées historiquement autour de l'étude des 
gaz à effet de serre, via ICOS, de la composition de l'atmosphère via IAGOS et plus récemment, de l'étude des aérosols et des gaz réactifs impliqués dans la pollution de l'air via ACTRIS. ACTRIS-FR fédère plusieurs SOERE et dispositifs élémentaires d'organismes de recherche. Le pôle Atmosphère (AERIS) du PÔLE DE DONNÉES rassemble tous les centres et bases de données préexistants des composantes d'ACTRIS-FR et de IAGOS.

\section{OCÉAN ET LITTORAL}

I-LICO, dédiée aux milieux littoral et côtier, regroupe des SOERE et des systèmes d'observation de plusieurs établissements; elle s'est construite dans la logique européenne du projet Jerico. A terme, le domaine océanique sera entièrement couvert par les infrastructures d'observation EMSO, EURO-ARGO et I-LICO avec l'ajout potentiel d'une infrastructure dédiée à la haute mer construite autour du SOERE «Coriolis-temps-différé-Observations-Océaniques». Le pôle OCEAN du PÔLEDEDONNÉES intégrera les données de I-LICO.

\section{ZONE CRITIQUE DES SURFACES CONTINENTALES} ○

OZCAR est une infrastructure d'observation ayant pour objet d'étude la Zone Critique (zone d'interactions entre lithosphère, hydrosphère, cryosphère, biosphère et atmosphère) afin d'appréhender les mécanismes de stockage et de transfert d'énergie et de matière (eau, carbone...) sur différentes échelles de temps et sous différentes contraintes. OZCAR permet une structuration nationale renforcée du domaine puisqu'elle associe plusieurs SOERE et des dispositifs d'observation des organismes de recherche. Les données contribueront au pôle Surfaces Continentales (THEIA) du PÔLE DE DONNÉES. Par ailleurs, OZCAR participe, aux côtés du Réseau des Zones Ateliers ${ }^{2}$ aux discussions préparatoires de I'ESFRI en projet e-LTER (Long Term Ecological Research network).

\section{BIODIVERSITÉ, ÉCOSYSTÈMES} ○

La cartographie des outils dédiés montre un pavage progressif par des infrastructures déjà bien organisées. Le

2 En dehors de la feuille de route, le SOERE Réseau des Zones ateliers (RZA) porte sur les écosystèmes, les pratiques humaines sur ces milieux, mais aussi les fonctionnalités écologiques. paysage s'articule actuellement autour de différents outils d'expérimentation, de collection (archivage d'échantillons), d'analyse mais aussi d'observation.

Deux infrastructures d'expérimentation permettent de conditionner des écosystèmes et de suivre les processus en jeu sous forçage contrôlé : AnaEE-FR (in natura) et ECOTRONS (mésocosmes). Ensemble, elles constituent le miroir français de l'ESFRI AnaEE.

Les infrastructures d'archivage d'échantillons sont: RARe rassemblant les centres de ressources biologiques animales, végétales et microbiennes dédiés à l'agronomie et RECOLNAT regroupant les collections naturalistes. Elles s'inscrivent dans une logique européenne (MIRRI pour les ressources microbiennes et le projet EU-Coll pour les collections). Elles sont porteuses d'enjeux stratégiques s'agissant de l'utilisation des ressources biologiques à des fins de recherche et développement.

ECOSCOPE a pour objet l'accès aux données d'observation, de collection et d'expérimentation, en lien avec le réseau européen EU-BON (Biodiversity Observatory Network) et I'OI GBIF (Global Biodiversity Information Facility). Il permet aussi la mise en réseau des observatoires de recherche de la biodiversité.

Les questions de recherche se situant entre Milieux et Vivant, certaines infrastructures sont partagées avec le domaine Biologie/Santé. EMPHASIS-FR, plateforme de phénotypage des plantes, est le miroir de l'ESFRI du même nom. Elle complète pour les agronomes et les écologues des plateformes "omiques" plutôt portées par Aviesan, comme Metabohub. Deux infrastructures sont d'un grand intérêt pour les sciences de l'environnement : il s'agit d'une part, de IBISBA-FR pour le développement de la biologie de synthèse et d'autre part, de EMBRC-FR (miroir d'ESFRI) pour les ressources biologiques marines.

\section{INFRASTRUCTURES VIRTUELLES EN OBSERVATION DE LA TERRE}

-

CliMERI-FR a pour mission la réalisation de simulations numériques de référence pour le programme mondial de recherches sur le climat (WCRP). Au niveau national, elle vise à coordonner les grands modèles de Météo-France et de I'IPSL ${ }^{3}$, à les évaluer et à produire des projections du climat futur.

Le PÔLE DE DONNÉES est une infrastructure en projet qui ambitionne d'offrir un portail unique vers les quatre pôles AERIS, OCEAN, FORM@TER et THEIA ainsi que de nouveaux services et outils. Il permettra de gérer le cycle complet des données (mesures terrain et satellites) depuis leur production jusqu'à leur mise à disposition et l'alimentation des bases de données nationales, européennes et internationales (Copernicus, GEOSS...). Il est attendu une ouverture vers un pôle de données sur la biodiversité à partir d'ECOSCOPE.

3 Fédération Institut Pierre-Simon Laplace. 


\section{LISTE DES INFRASTRUCTURES DE RECHERCHE 4 DANS LE DOMAINE SCIENCES DU SYSTĖME TERRE ET ENVIRONNEMENT}

\begin{tabular}{|c|c|c|c|}
\hline CATÉGORIE & NOM & NOM COMPLET & ESFRI \\
\hline Ol & CEPMMT & $\begin{array}{l}\text { Centre Européen pour les Prévisions } \\
\text { Météorologiques à Moyen Terme }\end{array}$ & \\
\hline TGIR & Concordia & Base antarctique franco-italienne & \\
\hline TGIR & ECORD/IODP & $\begin{array}{l}\text { Programme international de forage profond } \\
\text { en mer/ } \\
\text { European Consortium for Ocean Drilling Research/ } \\
\text { International Ocean Discovery Program }\end{array}$ & \\
\hline TGIR & EURO-ARGO & $\begin{array}{l}\text { Réseau in-situ global d'observation des océans/ } \\
\text { European contribution to Argo programme }\end{array}$ & $\begin{array}{l}\text { EURO-ARGO } \\
(2006)\end{array}$ \\
\hline TGIR & FOF & Flotte Océanographique Française & \\
\hline TGIR & ICOS & $\begin{array}{l}\text { Système Intégré d'Observation du Carbone/ } \\
\text { Integrated Carbon Observation System }\end{array}$ & $\operatorname{ICOS}(2006)$ \\
\hline IR & ACTRIS - FR & $\begin{array}{l}\text { Aerosol, Cloud and Trace Gases Research } \\
\text { Infrastructure - France }\end{array}$ & ACTRIS (2016) \\
\hline IR & ANAEE - FR & $\begin{array}{l}\text { Analyses et Expérimentations sur les Ecosystèmes } \\
\text { - France }\end{array}$ & ANAEE (2010) \\
\hline IR & ClimERI-FR & $\begin{array}{l}\text { Infrastructure nationale de modélisation } \\
\text { du système climatique de la Terre/ } \\
\text { Earth's Climate system Modelling }\end{array}$ & \\
\hline IR & ECOSCOPE & $\begin{array}{l}\text { Pôle de données d'observation pour la recherche } \\
\text { sur la biodiversité }\end{array}$ & \\
\hline IR & ECOTRONS & Ecotrons & ANAEE (2010) \\
\hline$I R$ & $E M B R C-F R^{1}$ & $\begin{array}{l}\text { Centre National de Ressources Biologiques } \\
\text { Marines }\end{array}$ & EMBRC (2008) \\
\hline IR & EMPHASIS France 2 & $\begin{array}{l}\text { European Multi-environment Plant pHenomics } \\
\text { And Simulation InfraStructure -France }\end{array}$ & EMPHASIS (2016) \\
\hline IR & EMSO - France & $\begin{array}{l}\text { European Multidisciplinary Seafloor } \\
\text { and water column Observatory - France }\end{array}$ & EMSO (2006) \\
\hline IR & IAGOS - France & $\begin{array}{l}\text { Instruments de mesure embarqués sur avions } \\
\text { pour l'observation globale/ } \\
\text { In-service Aircraft for Global Observing System }\end{array}$ & IAGOS (2006) \\
\hline IR & I-LI-CO & Infrastructure LIttorale et COtière & \\
\hline IR & OZCAR & $\begin{array}{l}\text { Observatoire de la Zone Critique, } \\
\text { Applications, Recherche }\end{array}$ & E-LTER (2016) \\
\hline IR & RARE & Ressources Agronomiques pour la Recherche & \\
\hline
\end{tabular}

1 Relève également du secteur «Biologie et Santé », dans lequel la fiche de l'infrastructure est présentée.

2 Relève également du secteur "Biologie et Santé ». 


\begin{tabular}{|c|l|l|l|}
\hline CATÉGORIE & \multicolumn{1}{|c|}{ NOM } & \multicolumn{1}{c|}{ NOM COMPLET } & ESFRI \\
\hline IR & RECOLNAT & Réseau des Collections Naturalistes françaises \\
\hline R & RESIF/EPOS & $\begin{array}{l}\text { Réseau sismologique et géodésique français/ } \\
\text { European Plate Observing System }\end{array}$ & EPOS (2008) \\
\hline IR & SAFIRE & $\begin{array}{l}\text { Service des Avions Français Instrumentés } \\
\text { pour la Recherche en Environnement }\end{array}$ \\
\hline Projet & IBISBA-FR & $\begin{array}{l}\text { Indrial Biotechnology Innovation } \\
\text { and Synthetic Biology Accelerator }\end{array}$ \\
\hline Projet & Pole de données & Pôle de données et services pour le Système Terre \\
\hline
\end{tabular}

3 Relève également du secteur "Biologie et Santé », dans lequel la fiche de l'infrastructure est présentée. 


\section{Centre européen pour les prévisions météorologiques à moyen terme}

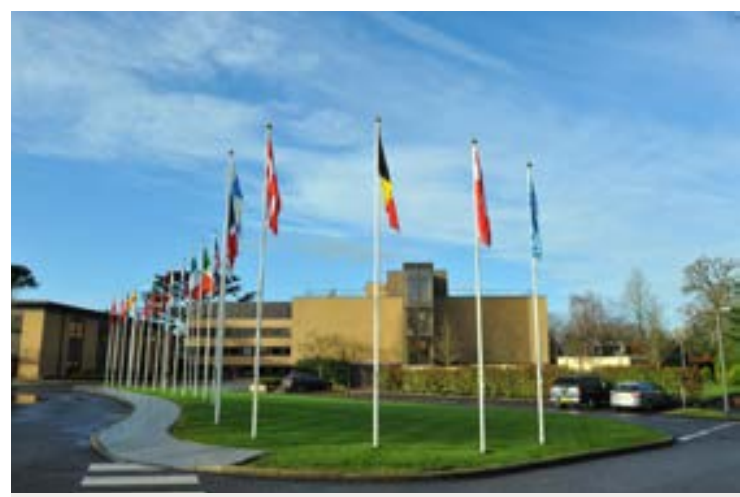

Type de l'infrastructure : OI

Statut de l'infrastructure :

Organisation Internationale

\section{Localisation : \\ Reading, UK}

Représentant en France :

Florence RABIER

Création: Exploitation:

1975

1979

\section{Pays partenaires :}

FR (P), EM, DE, AU, BE, DK, SP, FI, EL, IE, IS, IT, LU, NO, NL, PR, UK, RS, SI, SE, SW, TR. Etats coopérants : MK, BG, HR, EE, HU, IL, LV, LT, MA, $M E, C Z, R O, S K$

\section{Contact en France :}

www.ecmwf.int/en/about/contact-us

\section{www.ecmwf.int}

Le Centre européen pour les prévisions météorologiques à moyen terme (ECMWF) est une organisation intergouvernementale indépendante financée par 34 Etats.

Le CEPMMT est à la fois un institut de recherche et un service opérationnel $24 \mathrm{~h} / 24,7 \mathrm{j} / 7$, qui produit et diffuse à ses Etats membres des prévisions numériques du temps. Ces données sont intégralement mises à la disposition des chercheurs et des services météorologiques nationaux des Etats membres. Le Centre propose également un catalogue de produits de prévision pouvant être achetés par les entreprises du monde entier ou d'autres clients commerciaux. L'installation de supercalcul du CEPMMT (et les archives de données associées) est l'une des plus importantes en son genre en Europe, et les Etats membres peuvent utiliser $25 \%$ de sa capacité à leurs propres fins.

L'organisation a été créée en 1975 et emploie aujourd'hui près de 280 membres du personnel provenant de plus de 30 pays. Le CEPMMT est I'un des six membres des Organisations coordonnées, qui comprennent également l'Organisation du traité de l'Atlantique Nord (OTAN), le Conseil de l'Europe (COE), l'Agence spatiale européenne (ESA), I'Organisation de coopération et de développement économiques (OCDE) et l'Organisation européenne pour l'exploitation de satellites météorologiques (EUMETSAT). Le siège du CEPMMT se trouve à Reading, au Royaume-Uni.

\section{IMPLICATIONSSOCIO-ÉCONOMIQUES}

Météorologie, Services d'Alertes et de Prévisions

\section{DONNÉES}

Présence dans des réseaux de données : EUROSIP : système de prévision saisonnière multimodèle fondé sur les prévisions du CEPMMT, du Met Office, de Météo-France et du NCEP

Archivage de prévisions d'ensemble d'une dizaine de centres mondiaux fournies dans le cadre du projet TIGGE de I'OMM

Hébergement de la plateforme du système d'information EFAS (European Flood Forecasting system), un service Copernicus

\section{Coût de fonctionnement \\ 63,4 M€ \\ dont 8,7 M€ de contribution française}

\section{Coût RH}

3,9 M€ 


\section{Base antarctique franco-italienne}

Concordia est une base de recherche polaire franco-italienne implantée en Antarctique. Seule station européenne au cœur du continent antarctique, elle a pour vocation majeure d'offrir à la communauté scientifique un accès au haut plateau antarctique. La TGIR inclut également l'environnement logistique nécessaire à l'approvisionnement de la station : le navire L'Astrolabe, la base annexe de Cap Prudhomme et les moyens de transport terrestres.

Les spécificités du site lui confèrent un caractère unique

- 3200 m d'altitude

- température moyenne de l'air à $-50^{\circ} \mathrm{C}$.

- positionnement sous la trace des satellites à orbite polaire;

- 3240 mm d'épaisseur de glace;

- positionnement sous le vortex polaire.

Concordia accueille 60 chercheurs et techniciens en été et 14 en hiver. Neuf mois par an, la base est isolée du reste du monde. Les recherches conduites à Concordia viennent renforcer les observations de la planète sur unvaste continent quine compte que 3 stations de recherche intérieures. Elle permet la réalisation de programmes de recherche et d'observation uniques dans de nombreux domaines scientifiques et technologiques (glaciologie, physique et chimie de l'atmosphère, astronomie, géophysique...), dont bon nombre en lien avec les changements climatiques. Elle abrite également des études soutenues par l'Agence Spatiale Européenne (ESA) sur la médecine et le comportement humain en milieu confiné. Au-delà de cette valeur scientifique, Concordia remplit un rôle géopolitique important en confortant la présence française en Antarctique et en en faisant l'un des principaux acteurs de la recherche scientifique sur le 6e continent.

\section{IMPLICATIONSSOCIO-ÉCONOMIQUES}

Certaines activités en lien avec la logistique et la maintenance de la station sont sources de développements technologiques. Par exemple :

- développement du mode de transport par convois terrestres sur glace (IPEV leader mondial),

- développements de systèmes de traitements d'eaux usées (collaboration avec l'ESA),

- développement des énergies renouvelables (énergie solaire).

\section{Coût de construction}

34 M€ dont 17 M€ de

contribution française

Cout de fonctionnement :

10,6 M€ dont $50 \%$ de

contribution française

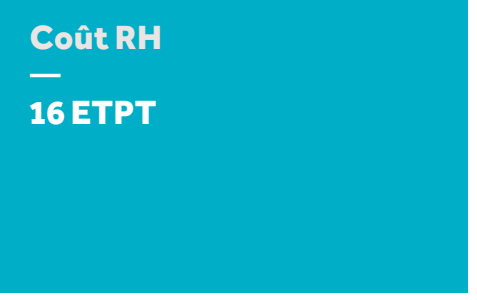

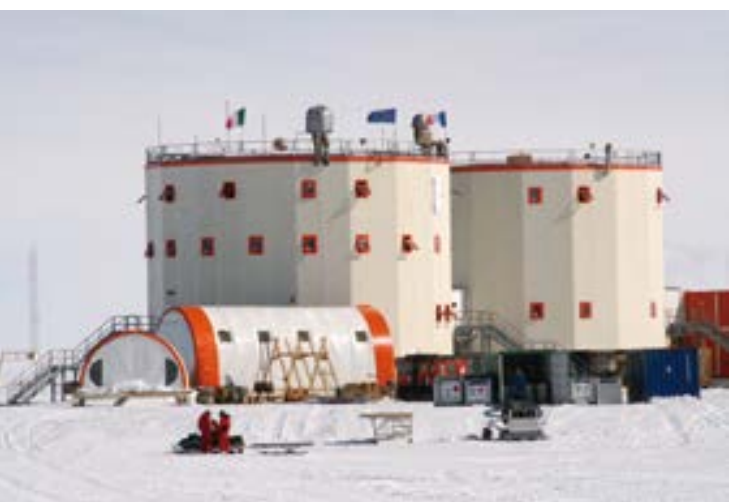

Type de l'infrastructure : TGIR

\section{Localisation:}

"Dome C", Antarctique ( $\left.75^{\circ} 06^{\prime} \mathrm{S}-123^{\circ} 21^{\prime} \mathrm{E}\right)$

sur le plateau continental à $1100 \mathrm{~km}$ de la base

française «Dumont d'Urville »

\section{Responsable de l'infrastructure :}

Yves FRENOT

\section{Création :}

\section{Exploitation :}

1998

2005

\section{Tutelles / Partenaires:}

GIPIPEV (MENESR, MAEDI, CNRS, CEA,

CNES, Ifremer, Météo-France, TAAF, EPF),

Programma Nazionale di Ricerche in Antartide

(PNRA) - Italie ( MIUR, ENEA, CNR)

\section{Contact en France :}

dirpol@ipev.fr

www.institut-polaire.fr/ipev/

infrastructures/les-bases/

concordia/

\section{Dimension internationale}

Pays partenaires : FR, IT

Site internet :

www.enea.it/en/research-development/climate-and-the-environment/ expeditions-in-antarctica-and-research-in-polar-areas 


\section{Programme international de forage profond en mer/ European Consortium for Ocean Drilling Research/ International Ocean Discovery Program}

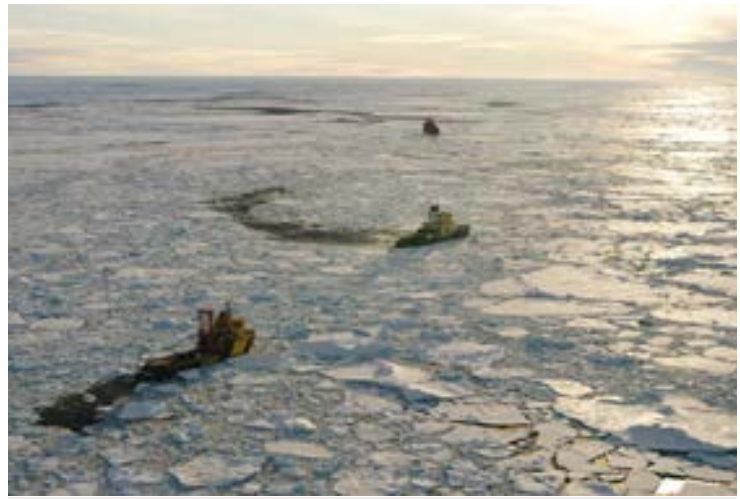

Type de l'infrastructure: TGIR

Localisation de l'infrastructure:

Aix-en-Provence (coordination)

\section{Localisation des autres sites :}

IR distribuée

Responsable de l'infrastructure :

Gilbert CAMOIN

Création: Exploitation:

$2013 \quad 2013$

\section{Tutelles / Partenaires :}

CNRS

\section{Contact en France:}

camoin@cerege.fr

ema@cerege.fr

\section{www.ecord.org}

www.iodp-france.org
ECORD est un consortium européen. II participe au programme IODP (International Ocean Discovery Program; 25 pays) et donne à la communauté française l'accès à trois types de plate-forme de forage dans un cadre international

- les Etats-Unis opèrent un navire de forage conventionnel, le JOIDES Resolution:

- I'opérateur japonais CDEX met en œuvre le Chikyu qui est équipé d'un système de recirculation de boues et qui permet de forer jusqu'à 6-7 km et dans les formations géologiques instables;

- ECORD met en œuvre des plates formes spécifiques «MSP» (Mission Specific Platforms), contractées au coup par coup, pour forer les environnements inaccessibles aux deux autres navires, en particulier les zones englacées et les eaux peu profondes.

Le concept de MSP est maintenant étendu à d'autres moyens de forages (foreuses téléguidées, carottiers à sédiments etc.), plus économiques que des navires de forages conventionnels et mieux adaptés à certains types d'environnements et d'opérations.

Le forage océanique et les techniques associées sont des outils essentiels et indispensables pour comprendre et prédire le fonctionnement du système Terre. Le programme IODP aborde quatre grands thèmes scientifiques:

1. changements climatiques et environnementaux;

2. biosphère : vie en subsurface océanique, biodiversité et forçage environnemental des écosystèmes:

3. processus profonds et impact sur les environnements superficiels;

4. Terre en mouvement: processus et risques à l'échelle humaine.

\section{IMPLICATIONS SOCIO-ÉCONOMIQUES}

Les forages scientifiques fournissent des données essentielles à différents secteurs industriels (industrie pétrolière, biotechnologies, etc.). ECORD et IODP innovent en termes de développement technologique concernant le matériel et les techniques de forages en collaboration avec le secteur industriel (grands entreprises et PME) et dans le cadre de pôles de compétitivité.

\section{DONNÉES}

Estimation du flux de données : Plusieurs To/an

Accessibilité : Toutes les données et les carottes récoltées au cours des campagnes sont stockées dans trois carothèques (College Station, US; Kochi, JP; Brême, EU) et sont en accès ouvert à la communauté scientifique après une période moratoire d'un an.

\section{Coût de fonctionnement \\ - \\ 4,5 M€}

\section{Coût RH}

$-$

\section{ETPT}

\section{Dimension internationale \\ Coordinateur : Gilbert Camoin, FR, pour le consortium ECORD \\ Pays partenaires : IODP = consortium ECORD (DE, AT, BE, CA, DK, ES, FI, FR, IE, IL, IT, NO, NL, PL, PT, UK, SE, CH) + US, BR, CN, KR, IN, AU, NZ \\ Site internet : www.ecord.org, www.iodp.org}




\section{Réseau in-situ global d'observation des océans/ European contribution to Argo programme}

Argo est un réseau international de plus de 3500 flotteurs profilants qui mesurent en temps réel la température et la salinité des océans de la surface à $2000 \mathrm{~m}$ de profondeur. C'est le premier réseau in-situ global d'observation des océans en temps réel, complément indispensable des systèmes satellitaires, pour observer, comprendre et prévoir l'océan et son rôle sur le climat. Les objectifs principaux d'Argo sont de consolider et pérenniser le réseau actuel sur les 10 à 20 prochaines années.

La caractérisation du changement climatique et du rôle fondamental de l'océan ne peut se faire qu'à partir d'observations obtenues dans la durée. Des évolutions seront progressivement apportées : couverture des zones polaires et autres mers, développements technologiques, extension aux plus grandes profondeurs et ajout de capteurs biogéochimiques. Dans ce contexte, l'objectif d'Euro-Argo est d'organiser les contributions de ses membres afin de permettre àl'Europe de

- déployer, maintenir et opérer un réseau de plus de 800 flotteurs soit plus de 250 flotteurs nouveau chaque année:

- fournir un service d'excellence aux communautés recherche (océan, climat) et océanographie opérationnelle (Service Marin de Copernicus); - préparer et contribuer àla nouvelle phase d'Argo avecune extensionaux paramètres biogéochimiques, aux plus grandes profondeurs et auxzones polaires.

\section{IMPLICATIONSSOCIO-ÉCONOMIQUES}

Les impacts socio-économiques concernent une vaste gamme de services océaniques développés via le programme européen Copernicus et son service marin. D'importants partenariats existent avec des PME sur la technologie marine et les capteurs, en particulier avec la PME française NKE qui est le principal producteur industriel de flotteurs Argo en Europe.

\section{DONNÉES}

Estimation du flux de données : Volume $<100 \mathrm{MO} /$ jour

Stockage : Le volume total des données Argo est environ 500 GO.

Accessibilité : via le Centre de données Coriolis, et diffusées en temps réel sur le réseau du Service Mondial de Télécommunication de l'Organisation Mondiale de la Météorologie. Les données sont disponibles sur www.argodatamgt.org/Access-to-data. Environ 1300 utilisateurs distincts accèdent chaque mois aux données.

Présence dans des réseaux de données : Coriolis, Mercator Océan Infrastructures marines européennes (SeaDataNet et EMODnet, Copernicus Marine Service). Programme international Argo (GOOS).

\section{Coût de fonctionnement \\ - \\ 2,2 M€ \\ dont 12 ETPT}

\author{
Dimension internationale \\ EURO-ARGO ERIC, ESFRI Landmark \\ Coordinateur : Yves Le Traon, FR \\ Pays partenaires : DE, UK, IT, NL, GR, FI, NO, PL \\ Site internet : www.argo.ucsd.edu / www.jcommops.org/argo
}

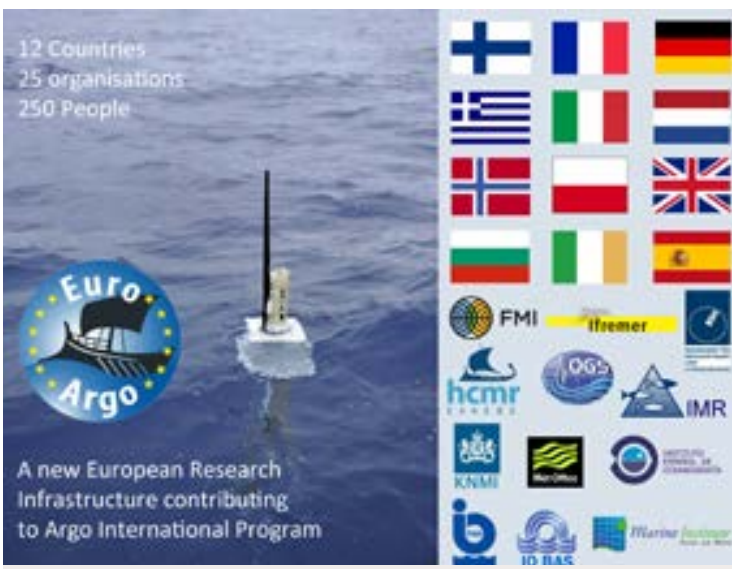

Type de l'infrastructure : TGIR

Localisation:

Brest (coordination)

Localisation des autres sites :

IR distribuée

Responsables de l'infrastructure :

Sylvie POULIQUEN, Pierre-Yves LE TRAON

\begin{tabular}{|r|r|}
\hline Création : & Exploitation : \\
\hline 2001 & 2011 \\
\hline
\end{tabular}

\section{Jouvence :}

Déploiement régulier de nouveaux flotteurs chaque année.

\section{Tutelles / Partenaires :}

Ifremer, CNRS, SHOM, Météo-France, IRD, CNES, IPEV (Consortium Coriolis)

\section{Contact en France :}

sylvie.pouliquen@ifremer.fr

\section{www.euro-argo.eu}




\section{Flotte Océanographique française}

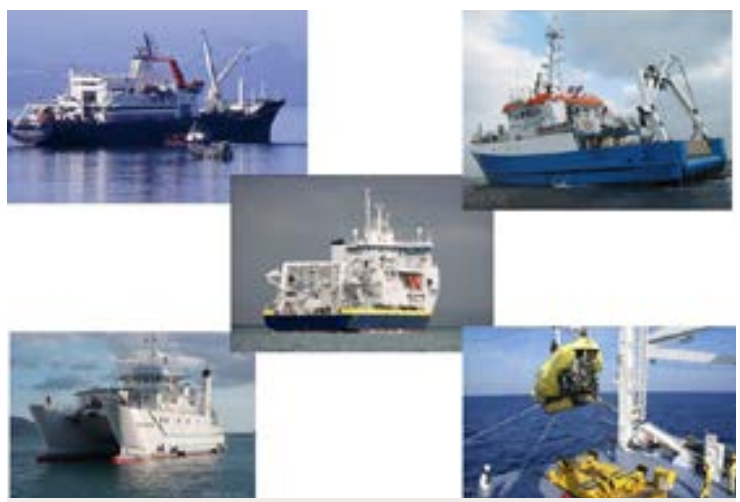

Type de l'infrastructure : TGIR

\section{Statut de l'infrastructure:}

Unité mixte de service

\section{Localisation :}

Toulon, Brest (coordination)

\section{Localisation des autres sites :}

IR distribuée

Responsable de l'infrastructure :

Olivier LEFORT

\begin{tabular}{cc|} 
Création: & Exploitation : \\
\hline 2011 & 1960 \\
(pourl'UMS) & (premiers navires)
\end{tabular}

\section{Jouvence:}

Plan d'évolution de la flotte planifié sur 20 ans

\section{Tutelles / Partenaires :}

CNRS, IFREMER, IRD, IPEV,

Marine Nationale, TAAF

\section{Contact en France :}

olivier.lefort@ifremer.fr

\section{www.flotteoceanographique.fr}

La flotte océanographique française fédère les navires de recherche nationaux qui permettent de mener en milieu marin côtier et hauturier des recherches en géosciences, océanographie physique et biologique, biogéochimie des océans, paléoclimatologie, biodiversité... Elle compte:

- 5 navires hauturiers: Atalante, Pourquoi Pas?. Thalassa, Marion Dufresne, le Suroit);

- 2 navires en outremer: Alis, Antea;

- 5 navires côtiers utilisés en Manche-Atlantique et en Méditerranée Thetys, L'Europe, Thalia, Côtes de la Manche, Haliotis:

- des navires de station répartis sur les façades maritimes métropolitaines, qui réalisent des sorties de 1 à 3 jours;

- des engins sous-marins: Nautile(habité), robot télé-opéré Victor6000, AUV - des instruments scientifiques : sismique, pénétromètre Penfeld, carottier.

Elle participe à la formation par et pour la recherche. Elle contribue à des missions de service public de surveillance et d'expertise : hydrographie, environnement côtier, ressources halieutiques, biodiversité, évaluation de risques naturels (sismique, volcanique, gravitaire, tsunami).

Types d'opérations et d'observations:

- mesures physico chimiques de la colonne d'eau;

- bathymétrie et cartographie, sismique;

- imagerie;

- prélèvements et analyse d'échantillons (eau, faune, flore, carottage sédiments jusqu'à 70 mètres de profondeur, roches et minerais affleurant);

- installation et entretien d'observatoires de fond de mer:

- mesures en route (météorologie, courantométrie, géophysique...).

\section{IMPLICATIONSSOCIO-ÉCONOMIQUES}

Partenariats pour la conception, la construction et l'entretien de navires, d'engins sous-marins, de capteurs et d'instruments scientifiques embarqués.

Partenariats scientifiques dans les domaines des technologies marines et sous-marines (capteurs, robots autonomes, énergies renouvelables), des biotechnologies, des ressources minérales (sulfures polymétalliques) et énergétiques (pétrole notamment).

\section{DONNÉES}

Stockage : Centre de données à Brest (Ifremer) et Pôle de données et de services OCEAN

Accessibilité : Via le site www.flotteoceanographique.fr/Documentation Présence dans des réseaux de données : Portails nationaux : Sismer (Ifremer), Pôle de données et de services OCEAN (cf. IR en projet Pôle de données)

Portails européens : Coriolis (www.coriolis.eu.org), SeaDataNet (www.seadatanet.org), Emodnet (www.emodnet.eu)

\section{Coût de construction \\ 600 M€ (estimation \\ de la valeur à neuf) \\ Coût de fonctionnement : $75 \mathrm{M€}$}

\section{Coût RH}

\section{ETPT}

\section{Dimension internationale \\ "Ocean Facilities Exchange Group », Eurofleets}

Site internet : www.ofeg.org, www.eurofleets.eu 


\section{Système Intégré d'Observation du Carbone/ Integrated Carbon Observation System}

ICOS est une IR distribuée fournissant des mesures harmonisées à l'échelle européenne sur le cycle du carbone, les émissions et les concentrations atmosphériques des gaz à effet de serre (GES). ICOS intègre des réseaux de mesures dans l'atmosphère, au niveau des écosystèmes terrestres et de l'océan.

Chaque réseau est coordonné par son Centre thématique (CT) responsable du traitement des données et du contrôle qualité. Les CT Atmosphere, Ecosystème et Océan sont respectivement coordonnés par la France, I'Italie et la Norvège. Deux autres centres basés en Allemagne fournissent les gaz de référence pour les réseaux et effectuent des analyses d'échantillons d'air prélevés par flacons. Les headquarters sont basés en Finlande et un portail carbone est situé en Suède.

La mission de recherche d'ICOS est de fournir les observations nécessaires pour comprendre les échanges de GES et pour prédire le comportement futur des puits de carbone et les émissions des GES.

Ainsi l'objectif d'ICOS est de mesurer sur le long terme les paramètres essentiels du cycle du carbone et des principaux GES. Le jeu de données cohérent obtenu permet la recherche sur les flux de GES et sur les processus multi-échelles qui les déterminent. ICOS permettra de détecter les changements dans les flux régionaux de GES, de mesurer l'impact des événements climatiques extrêmes et des politiques de réduction des émissions, de réduire les incertitudes au sein des modèles du système Terre et de leurs prédictions.

La participation de la France (ICOS-FR) se fait essentiellement au travers des CT Atmosphère et Ecosystèmes.

\section{DONNÉES}

Accessibilité : Les données ICOS sont publiques, ouvertes à tous les utilisateurs

\section{Coût de construction}

$18 \mathrm{M} €$

Coût de fonctionnement : $7 \mathrm{M€}$

\section{Dimension internationale}

ICOS ERIC, ESFRI Landmark

Coordinateur : Kutsch Werner, FI

Pays partenaires: Membres : DE, BE, FI, FR, IT, NO, NL, SE, Observateur : $\mathrm{CH}$

Site internet : $\underline{w w w . i c o s-r i . e u}$

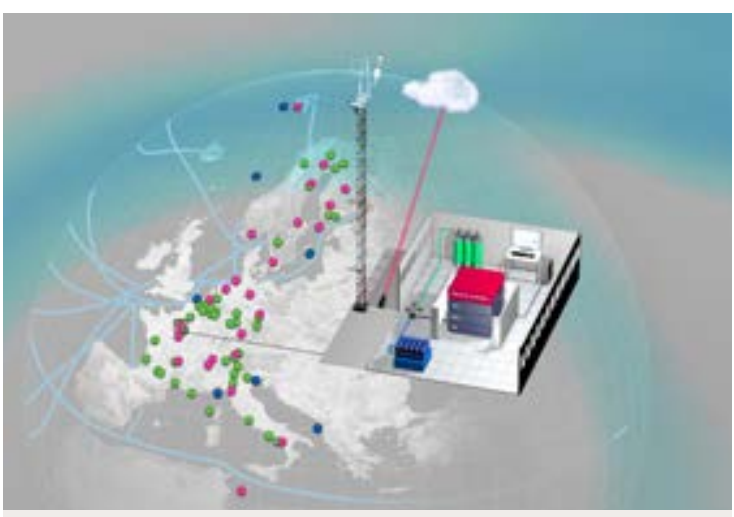

Type de l'infrastructure : TGIR

\section{Localisation:}

Saclay (coordination)

Localisation des autres sites:

IR distribuée

Responsable de l'infrastructure:

Philippe CIAIS

\section{Création :}

\section{Exploitation :}

2013 2016

Upgrade en moyenne tous les 5 à 7 ans

\section{Tutelles / Partenaires :}

CEA, CNRS, INRA, Univ. Versailles St-Quentin, Observateur: Andra, Collaborations: Universités Avignon, Aix-Marseille, Antilles, Bordeaux, Clermont-Ferrand, Guyane, La Réunion, Montpellier, Orléans, Paris-Saclay, Paris-Sud, Paris 6, Reims, Toulouse

\section{Coordinateur de l'infrastructure internationale :}

France, Etienne SNOECK (CNRS-CEMES)

\section{Contact en France :}

philippe.ciais@lsce.ipsl.fr

icos-atc.Isce.ipsl.fr

Icos-eco.fr 


\section{ACTRIS-FR}

\section{Aerosol, Cloud and Trace Gases Research Infrastructure}

\section{- France}

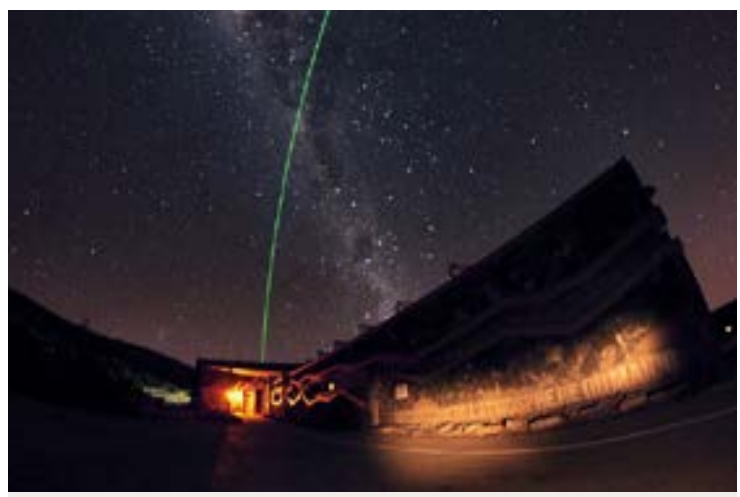

Type de l'infrastructure: IR

\section{Localisation : \\ Paris (coordination) \\ Localisation des autres sites : \\ IR distribuée}

\section{Responsable de l'infrastructure : \\ Jean-François DOUSSIN}

\section{Création :}

\section{Exploitation :}

2016

$$
2018
$$

\section{Tutelles / Partenaires :}

CNRS, ANDRA, CEA, CNES, GIPIPEV, INERIS, Météo France, IRSN, Univ. Grenoble-Alpes, Univ. Toulouse, Univ. Clermont-Ferrand, Institut Pierre-Simon-Laplace, Univ. Pierre et Marie Curie, Univ. Versailles St.-Quentin, Univ. de Lille, Univ. de la Réunion, Univ. Paris-Est Créteil Ecole Nationale Supérieure des Mines de Douai

\section{Contact en France :}

Jean-francois.doussin@cnrs-dir.fr
ACTRIS-FR est la composante française d'ACTRIS, l'initiative Européenne pour l'observation et l'exploration des aérosols, des nuages et des gaz réactifs et de leurs interactions. ACTRIS est une infrastructure de recherche distribuée, en support des recherches sur le climat et la qualité de l'air. Elle permet d'améliorer la compréhension de l'évolution passée, présente et future de la composition atmosphérique.

ACTRIS fournit des informations sur la variabilité 4-D des espèces à temps de vie court avec la précision et la qualité requises par les utilisateurs ainsi que des moyens d'exploration sur les processus atmosphériques.

ACTRIS opère des plateformes centrales (centres de données, centres de calibration) et fournit des services destinés à une large communauté d'utilisateurs travaillant sur les modèles chimie/climat, sur la validation des données satellitaires ou sur l'analyse de la prévision du temps ou de la qualité de l'air. Enfin, ACTRIS offre des modalités d'accès à des plateformes technologiques d'observation et d'exploration au service des communautés scientifiques et du secteur privé favorisant ainsi l'innovation technologique.

\section{IMPLICATIONSSOCIO-ÉCONOMIQUES}

Les technologies et les méthodes pour l'observation de l'atmosphère sont au cœur de l'IR ACTRIS, tant du point de vue de l'équipement que de l'algorithmique. ACTRIS a déjà établi un fort partenariat avec 11 entreprises privées intéressées par l'accès aux plateformes technologiques et de calibration et par la capacité de l'IR à définir des standards amenant à de nouvelles normes pour les instruments.

\section{DONNÉES}

\section{Stockage : 1 To}

Accessibilité : Pôle de données et de services AERIS (cf. IR en projet Pôle de données). Certains services pourront être disponibles uniquement aux membres du consortium

Présence dans des réseaux de données : Au niveau européen : Centre de données ACTRIS

\section{Coût de construction \\ - \\ $10 \mathrm{M€}$ \\ Coût de fonctionnement : 1,88 M€}

\author{
Dimension internationale \\ ACTRIS ESFRI Roadmap \\ Coordinateur: Markku Kulmala, FI \\ Pays membres: CY, CZ, FI, FR, GR, IE, IT, NL, PL, RO, ES, SE; UK ; \\ partenaires : BE, BG, DK, ES, DE, HU, NO, SE \\ Site internet: $\underline{w w w . a c t r i s . e u}$
}




\section{ANAEE-FR}

\section{Analyses et Expérimentations sur les Ecosystèmes}

\section{- France}

AnaEE-France "Analyse et Expérimentation sur les Ecosystèmes - France » est une infrastructure nationale de recherche dédiée à l'expérimentation sur les écosystèmes continentaux terrestres et aquatiques. Coordonnée par le CNRS et I'INRA, elle constitue la contribution française au projet européen AnaEE-Europe inscrit sur la feuille de route ESFRI.

Elle vise à comprendre et prédire la dynamique de la biodiversité et des écosystèmes grâce à une approche intégrée combinant les sciences du vivant et environnementales.

AnaEE-France réunit un ensemble de plateformes expérimentales offrant trois niveaux de contrôle des conditions environnementales : les mésocosmes en lien avec l'IR Ecotrons (2), les milieux semi ouverts permettant le contrôle partiel des flux (4) et des sites d'expérimentation in-natura (20) couvrant une variété d'écosystèmes (lac, forêt, prairie, culture) en France métropolitaine et en zone tropicale. Pour augmenter les capacités d'observation du milieu. AnaEE-France offre des plateformes analytiques et des instruments mobiles pouvant être déployés sur le terrain. Parallèlement aux moyens expérimentaux, AnaEE-France intègre des plateformes de modélisation permettant de mettre en œuvre des modèles existants ou d'en développer de nouveaux. Enfin pour promouvoir l'utilisation des données produites, AnaEE-France propose des systèmes d'information accessibles par internet.

Sur le plan collectif, AnaEE-France porte des actions sur l'harmonisation et la standardisation des méthodes, des mesures et des métadonnées.

\section{IMPLICATIONS SOCIO-ÉCONOMIQUES}

Les services d'AnaEE France établissent en moyenne une douzaine de nouveaux partenariats industriels chaque année. On peut citer par exemple le service PRO, en partenariat avec Veolia Environnement depuis 1998, pour l'évaluation des effets des apports de produits résiduaires organiques sur les agrosystèmes.

\section{DONNÉES}

Estimation du flux de données : plusieurs To/an

Stockage : Stockage des données produites sur un SI commun pour les sites d'expérimentations à long terme et archivage des métadonnées pour les expérimentations à façon menés sur les autres dispositifs

Accessibilité : Ouverture au monde académique après un délai de 1 à 5 ans Présence dans des réseaux de données : portail ECOSCOPE; ICOS

\section{Coût de construction \\ Entre 0,5 et $30 \mathrm{M} €$ par plateforme selon le type \\ Coût de fonctionnement \\ Entre 10 et $100 \mathrm{k} €$ par plateforme}

\author{
Dimension internationale \\ AnaEE ESFRI Roadmap \\ Coordinateur : Abad Chabbi, FR \\ Pays partenaires : FR, UK, NO, DK, TR, IT, CZ, BE, FI, SE, IL, EE, PO
}

Site internet : www.anaee.com

\section{Coût RH \\ Entre 1 et 4 ETPT par plateforme}

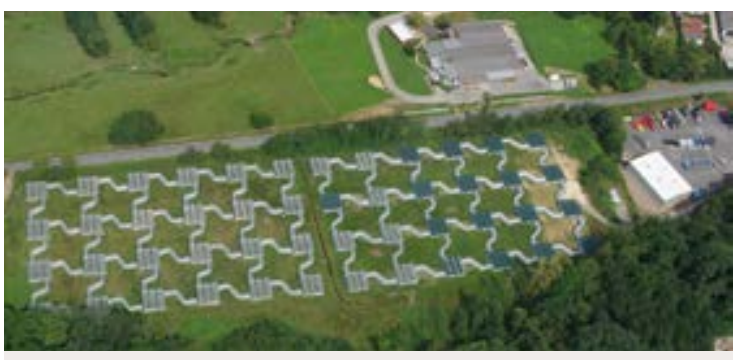

Type de l'infrastructure : IR

\section{Localisation:}

Moulis (coordination)

\section{Localisation des autres sites :}

IR distribuée : plusieurs sites en métropole, 2 sites en Guyane française, 1 site Brésil, 1 site Congo, 1 site Thaïlande

\section{Responsable de l'infrastructure :} Jean CLOBERT

\section{Création: Exploitation: \\ 2012 2012}

\section{Tutelles / Partenaires :}

CNRS, INRA, Univ. Grenoble.

Partenaires associés : ENS, Univ. Bourgogne, Univ. Savoie, Univ. Avignon

\section{Contact en France :}

jean.clobert@ecoex-moulis.cnrs.fr

\section{www.anaee-france.fr}




\section{CLIMERI-FRANCE}

\section{Infrastructure nationale de modélisation du système climatique de la Terre}

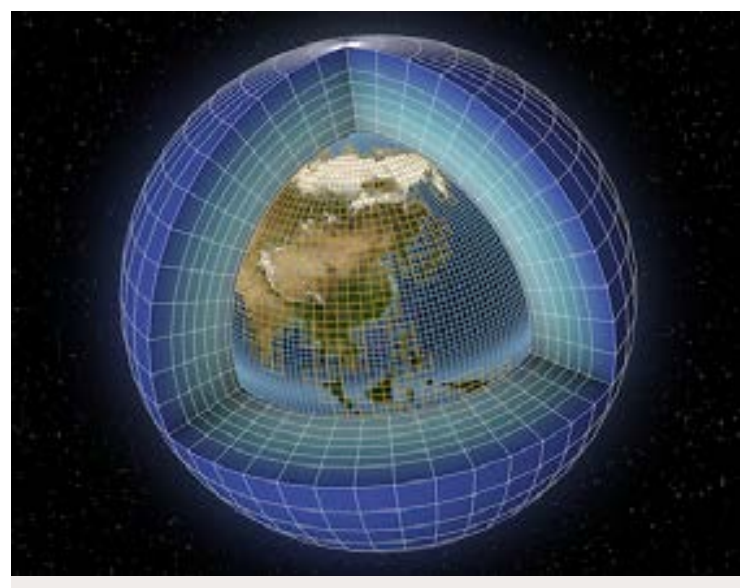

Type de l'infrastructure : IR

\section{Localisation :}

Paris-Saclay

\section{Localisation des autres sites :}

Toulouse

\section{Responsable de l'infrastructure :}

Sylvie JOUSSAUME

\section{Création: Exploitation: \\ 2016

2016

\section{Tutelles / Partenaires :}

CNRS, CEA, Météo-France, UPMC, IRD, Cerfacs, GENCl

\section{Contact en France :}

sylvie.joussaume@lsce.ipsl.fr
CliMERI-France est l'infrastructure nationale de modélisation du climat. Elle a pour mission la réalisation des simulations numériques internationales du Programme mondial de recherches sur le climat et la mise à disposition de leurs résultats pour divers utilisateurs. Ces expériences coordonnées visent à comprendre le fonctionnement du système climatique, à évaluer les capacités des modèles de climat par la définition d'expériences standard, à soutenir des études de mécanismes et de processus et à produire des projections de l'évolution future du climat. Ces expériences contribuent à l'élaboration des rapports du GIEC.

ClimERI-France inclut les ressources humaines assurant la mise au point et la maintenance des codes et des outils nécessaires à leur utilisation, la préparation des versions de référence des codes, à l'exploitation des données produites par les simulations, les ressources de calcul permettant la réalisation des expériences internationales ainsi que les capacités de stockage des données et l'infrastructure logicielle permettant la gestion et le contrôle du flux de données et de métadonnées.

L'infrastructure met à disposition des codes numériques et des outils logiciels. Elle offre un service d'accès aux données et d'analyse des résultats des modèles climatiques globaux, régionaux ainsi qu'aux simulations sur la France, dans le cadre du développement des services climatiques coordonné au niveau national par AllEnvi.

\section{IMPLICATIONSSOCIO-ÉCONOMIQUES}

Les projections climatiques sont une source d'information essentielle pour les politiques d'atténuation et d'adaptation au changement climatique. Elles sont utilisées par les services climatiques (AllEnvi, Copernicus), en particulier exploitées par des $\mathrm{PME}$ pour assister différents secteurs de l'économie (exemples : les projets KIC Climat: OASIS, E3P; projet ANR SECIF).

\section{DONNÉES}

Estimation du flux de données : 2 Po de données partagées à l'échelle internationale. Le nœud IPSL fournit de 10 à 30 To de données/mois. Le prochain exercice prévoit une mise à disposition des données de l'ordre de 40 Po, dont environ $10 \%$ par la France.

Stockage : 14 Po pour l'IPSL, 10 Po pour Météo-France.

Accessibilité : Les données françaises et européennes sont accessibles pour usage recherche et commercial.

Présence dans des réseaux de données : Base de données internationale des résultats de modèles : Earth System Grid Federation (ESGF)

\section{Coût de construction \\ - \\ $8 \mathrm{M€}$ \\ Coût de fonctionnement : 8,7 M€}

\section{Coût RH}

- 53 ETPT

\section{Dimension internationale \\ Dimension européenne en construction Earth's Climate System Modelling - European Research Infrastructure (CliM-ERI) \\ Coordinateur : Sylvie Joussaume, FR \\ Pays partenaires: FR, DE, IT, NL, NO, ES, SE, UK, IE, FI, DK}




\section{Pôle de données d'observation pour la recherche sur la biodiversité}

L'objectif scientifique est de documenter et comprendre l'état et la dynamique de la biodiversité et des services écosystémiques, de construire les scénarios du futur. Pour cela, ECOSCOPE favorise la complémentarité des observations de la biodiversité et la mise en relation de différents dispositifs d'observation et de recherche variant par l'amplitude spatiale et temporelle, les écosystèmes et règnes étudiés, les variables mesurées, le niveau d'organisation ainsi que les sources de données.

Pour répondre à cet enjeu, ECOSCOPE met en place un pôle de données d'observation pour la recherche sur la biodiversité, en concertation avec l'existant et en se basant sur le cycle de vie des données, pour faciliter les liens entre producteurs et utilisateurs de données et leur réutilisation.

Le pôle a pour objectifs

1. de fournir à une large communauté utilisatrice un accès à des métadonnées et jeux de données d'observation pour la recherche sur la biodiversité, des services et des outils de visualisation et d'analyses des données et des produits en promouvant la diffusion des bonnes pratiques;

2. de favoriser les réflexions autour du concept des «Essential Biodiversty Variables» (EBVs) pour saisir les principales dimensions de l'état et de la dynamique de la biodiversité:

3. de contribuer aux initiatives nationales, européennes et internationales sur les données et l'observation et favoriser l'ouverture à d'autres communautés de recherche ainsi qu'aux acteurs qui ont besoin d'expertise scientifique pour l'appui aux décisions publiques.

\section{IMPLICATIONS SOCIO-ÉCONOMIQUES}

Les observatoires impliqués dans ECOSCOPE concernent essentiellement des chercheurs académiques mais aussi des acteurs des sciences participatives encadrées par la recherche et des filières socioprofessionnelles travaillant avec la recherche, notamment sur les ressources génétiques domestiques animales, végétales et forestières. ECOSCOPE travaille aussi avec le Système d'Information sur la Nature et les Paysages (SINP), porté par le ministère en charge de l'Environnement, qui interagit avec les structures régionales de gestion et protection de la biodiversité.

\section{DONNÉES}

Estimation du flux de données : Plusieurs To/an

Stockage : Les données gérées dans le portail ECOSCOPE sont des métadonnées.

Accessibilité : Les métadonnées sont librement accessibles en ligne et les jeux de données correspondants autant que possible.

Présence dans des réseaux de données : ECOSCOPE est partenaire associé du réseau européen EU $B O N$; est labellisé « réseau national d'observation » par la composante «biodiversité (GEO BON) de GEOSS; établit des liens avec les systèmes d'informations nationaux et internationaux comme GeoCatalogue, MetaCat de DataONE, AnaEE-France, des dispositifs du CNRS-INEE grâce à BBEES, le GBIF-France, CRB-Anim, OCEANOMICS.

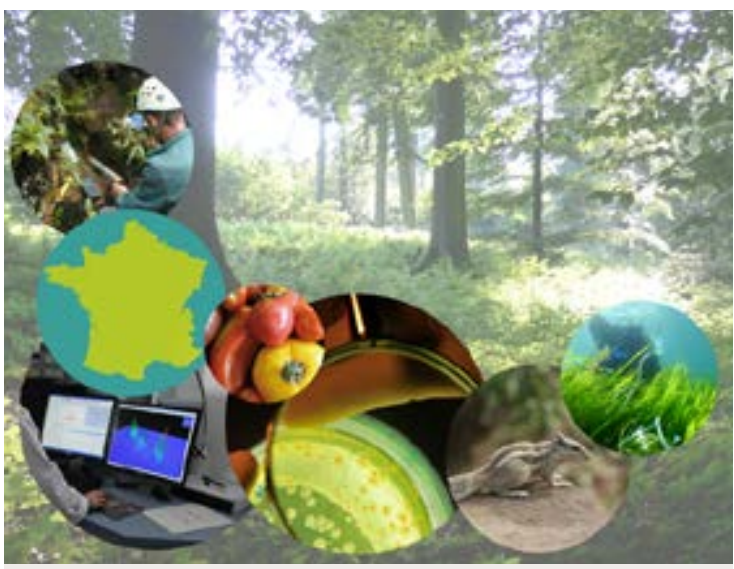

Type de I'infrastructure : IR

Localisation:

Paris (coordination)

Localisation des autres sites :

IR distribuée

Responsable de l'infrastructure : Aurélie DELAVAUD

Création:

Exploitation :

2011

2012

Tutelles / Partenaires:

FRB, BRGM, CIRAD, CNRS, IFREMER, INRA, IRD, IRSTEA, MNHN

\section{Contact en France :}

ecoscope@fondationbiodiversite.fr

\section{www.fondationbiodiversite.fr}

\section{ecoscope-net.fr}




\section{Ecotrons}

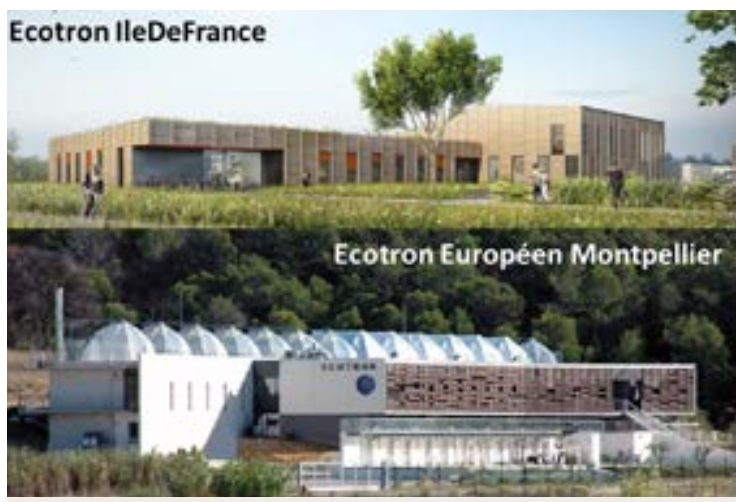

Type de l'infrastructure : IR

\section{Localisation : \\ Montpellier}

Localisation des autres sites :

Île-de-France (coordination)

\section{Responsable de l'infrastructure :}

Jacques ROY

\section{Création: Exploitation:}

2009 2011

\section{Tutelles / Partenaires :}

CNRS, ENS Paris/Conseil régional Île-de-France, Conseil régional Languedoc-Roussillon

\section{Contact en France :}

jacques.roy@cnrs.fr

\section{www.ir-ecotrons.cnrs.fr}

L'expérimentation sur des écosystèmes constitue un enjeu scientifique de premier plan qui demande de maîtriser la dynamique de systèmes complexes et adaptatifs ainsi que le contrôle et la mesure des paramètres physico-chimiques et biologiques associés. L'IR ECOTRONS met à la disposition d'une large communauté scientifique (écologie, agronomie, biologie, géosciences), nationale et internationale, deux Ecotrons avec leurs moyens humains, techniques, financiers, et organisationnels. Cette IR s'ouvre donc à toute la communauté des sciences du vivant et de l'environnement.

Au plan technologique, un Ecotron permet simultanément de conditionner l'environnement d'écosystèmes naturels, simplifiés ou complètement artificiels et de mesurer les états et l'activité des êtres vivants présents dans ces écosystèmes ainsi que le bilan intégré de cette activité, notamment en ce qui concerne les cycles biogéochimiques. Le principe est de confiner des écosystèmes dans des enceintes totalement ou partiellement étanches. Un nombre suffisant d'enceintes indépendantes est nécessaire pour assurer l'étude de plusieurs facteurs en interaction, dans un cadre d'inférence statistique.

Les Ecotrons sont les outils expérimentaux les plus évolués et complexes pour étudier les processus gouvernant la dynamique d'un système écologique complexe et adaptatif, analogue aux systèmes naturels ou complètement artificiels. Ce type d'approche expérimentale est nécessaire afin d'élaborer et de tester des concepts en écologie fonctionnelle et évolutive et d'apporter des éléments de réponse aux problèmes environnementaux actuels.

\section{IMPLICATIONSSOCIO-ÉCONOMIQUES}

Les grands secteurs de l'innovation concernés par l'IR sont les biotechnologies, l'agronomie, l'ingénierie écologique, les capteurs environnementaux et les équipements de conditionnement. L'infrastructure travaille avec les partenaires industriels et est informellement associée aux Pôles de compétitivité sur l'eau et sur le végétal. Un brevet est déposé tous les 1 ou 2 ans et plusieurs prix d'innovation ont été obtenus avec des entreprises françaises.

\section{DONNÉES}

Estimation du flux de données : 200 Mo par semaine

Stockage : 4 To

Accessibilité : en ligne

Présence dans des réseaux de données : Réseau AnaEE-France

\author{
Dimension internationale \\ AnaEE ESFRI Roadmap \\ Coordinateur: Abad Chabbi, FR \\ Pays partenaires : FR, UK, NO, DK, TR, IT, CZ, BE, FI, SE \\ Site internet: $\underline{w w w . a n a e e . c o m}$
}




\section{European Multi-environment Plant pHenomics And Simulation InfraStructure}

Emphasis-France a pour objectif de fournir les infrastructures nécessaires à l'analyse de la variabilité génétique des réponses des plantes aux stress environnementaux associés aux changements climatiques:

1. plateformes de phénotypage pour des milliers de plantes/parcelles, avec une définition temporelle allant de minutes à jours, ainsi que des capteurs de l'environnement ressenti par les plantes:

- plateformes robotisées équipées d'outils d'imagerie en conditions contrôlées, permettant des analyses architecturales et fonctionnelles; champs intensément instrumentés pour l'analyse de peuplement de plantes soumis à des situations artificialisées (enrichissement de l'air en $\mathrm{CO}_{2}$, sécheresse contrôlée) équipés de moyens intensifs d'imagerie du couvert:

- réseaux de champs équipés de capteurs et de moyens mobiles d'imagerie dans des conditions climatiques contrastées;

2. système distribué d'information conçu pour acquérir, organiser et stocker les données générées dans l'ensemble de l'infrastructure et pour échanger les données, en relation avec d'autres infrastructures;

3. outils d'analyse en termes

- d'évaluation haut débit de la qualité des millions de points de données

- d'analyses statistiques de données temporelles associées à des milliers d'individus:

4. outils de modélisation des plantes, capables d'extrapoler les données acquises dans des dizaines de scénarios climatiques européens à partir de paramètres recueillis sur les plateformes de phénotypage.

\section{IMPLICATIONS SOCIO-ÉCONOMIQUES}

Les relations sont fortes avec les semenciers et le développement agricole : mise à disposition de plateformes et de méthodes, information mutuelle stratégique et Industrial advisory board. Les technologies de phénotypage et d'agriculture de précision sont des marchés en émergence. Phenome/ Emphasis a abouti en trois ans à une spin-off, des brevets et la mise sur le marché de produits novateurs.

\section{DONNÉES}

Estimation du flux de données : 100 To/an

Stockage : 2-3 Po en 2020

Accessibilité : Minutes à semaines suivant les jeux de données

Présence dans des réseaux de données : France Grille, EU Grids

\section{Dimension internationale}

EMPHASIS ESFRI Roadmap

Coordinateur : Uli Schurr, DE

Pays partenaires : DE, FR, UK, BE

Site internet : En construction : $\underline{w w w . p l a n t-p h e n o t y p i n g . o r g / F a c t s ~}$

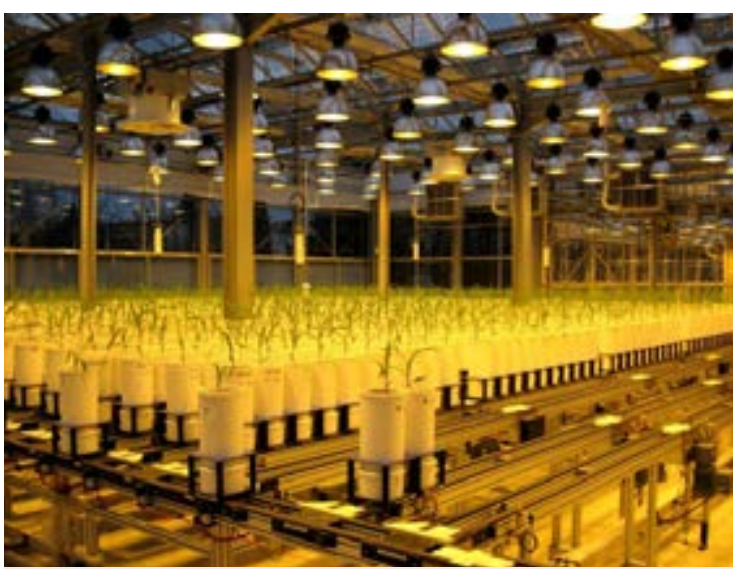

Type de l'infrastructure : IR

\section{Localisation:}

Montpellier (coordination)

Localisation des autres sites :

IR distribuée

\section{Responsable de l'infrastructure :} François TARDIEU

\begin{tabular}{|r|c|}
\hline Création : & Exploitation : \\
\hline 2012 & 2013 \\
\hline
\end{tabular}

\section{Tutelles / Partenaires:}

INRA, Arvalis Institut du Végétal, CEA, CNRS, Terres Inovia

\section{Contact en France :}

francois.tardieu@supagro.inra.fr

www.phenome-fppn.fr 


\section{European Multidisciplinary Seafloor and water column Observatory}

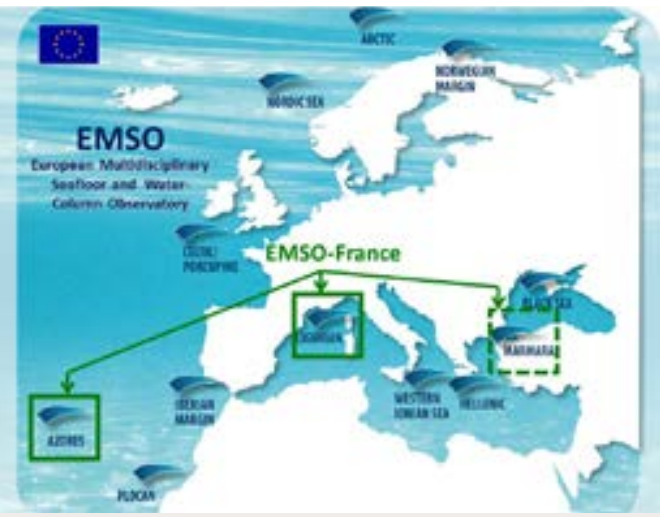

Type de l'infrastructure : IR

\section{Statut de l'infrastructure :}

Unité mixte de service

\section{Localisation :}

Paris, Brest (coordination)

\section{Localisation des autres sites :}

En mer : Mer Ligure, Açores, Mer de Marmara (en préparation)

\section{Responsables de l'infrastructure:}

Mathilde CANNAT, François ROLIN

\section{Création: Exploitation: \\ 1991 Echelonnée entre 1991 et 2017}

\section{Tutelles / Partenaires :}

CNRS, Ifremer

\section{Contact en France :}

cannat@ipgp.fr

jrolin@ifremer.fr

\section{www.emso-fr.org}

EMSO est une infrastructure distribuée qui met en oeuvre des observatoires du fond de mer et de la colonne d'eau. Elle est composée sur chaque site d'équipements de collecte de données d'observation sous-marine (capteurs physiques, chimiques, biologiques, caméras) et de liaisons câblées vers la côte ou mixtes acoustiques/hertziennes via des bouées, permettant la transmission en temps réel ou légèrement différé des données acquises. Une coopération des centres de données de Brest (Ifremer), Brême (Pangea) et Rome (INGV) permet un accès libre aux données par des portails disciplinaires et un accès temps réel.

Le réseau EMSO vise à acquérir des séries temporelles en milieu marin profond, avec pour objectifs scientifiques principaux

1. I'étude des processus sismiques, volcaniques, hydrothermaux et gravitaires, et le cas échéant des risques qui leur sont associés;

2. l'étude des écosystèmes marins profonds dans une optique de recherche fondamentale mais aussi de gestion durable, en particulier vis-à-vis des facteurs anthropogéniques et climatiques:

3. la contribution au suivi des changements globaux par l'acquisition de données eulériennes sur la colonne d'eau:

4. la promotion des développements en matière de technologies marines fonctionnant sous fortes pressions.

Les sites EMSO France sont: Mer de Marmara (en projet), Açores (en opération) et Mer Ligure (en opération et MEUST en 2016). L'IR intègre les fonctions communes du réseau.

\section{IMPLICATIONSSOCIO-ÉCONOMIQUES}

Des opportunités industrielles dans la fourniture d'infrastructure fixe câblée, ou déplaçables, d'instruments connectés, de constitutions de bases de données et de services associés. Le marché de la surveillance avant et pendant la production des champs pétroliers, de l'exploitation minière profonde et des EMR est visé. Promotion des PME via le projet inter-Carnot Captiven et les 2 Pôles de compétitivité Mer.

\section{DONNÉES}

Estimation du flux de données : $<0.5 \mathrm{To} / \mathrm{an}$. Toutefois, le flux augmente, en particulier par l'adjonction de caméras, sismomètres et échosondeurs.

Stockage : SISMER et RESIF

Accessibilité : Via des portails de chaque discipline et via les portails EMSO

Présence dans des réseaux de données : Pôle de données et de services OCEAN (cf. IR en projet Pôle de données)

\section{Coût de construction \\ 0,313 M€ \\ Coût de fonctionnement: 0,276 M€}

\section{Coût RH}

$1,340 \mathrm{M€}$

\section{Dimension internationale \\ ESFRI Landmark \\ Coordinateur : Paolo Favoli, INGV, IT \\ Pays partenaires : Membres : IT, UK, ES, RO, PT, GR, IE. Observ. : DE, NL \\ Site internet : www.emso-eu.org}




\section{Instruments de mesure embarqués sur avions pour l'observation globale/In-service Aircraft for Global Observing System}

IAGOS est une infrastructure de recherche européenne qui a pour but d'établir, d'opérer et d'exploiter un réseau global d'observation à long terme de la composition de l'atmosphère: gaz trace réactifs (ozone, monoxyde de carbone, oxydes d'azote), gaz à effet de serre (vapeur d'eau, dioxyde de carbone, méthane, ozone), aérosols et particules nuageuses (gouttes d'eau et cristaux de glace). A terme, la couverture globale sera assurée par une flotte d'une vingtaine d'avions de ligne Airbus appartenant à des compagnies aériennes internationales et embarquant l'instrumentation IAGOS

IAGOS est fondée sur l'expérience scientifique et technologique acquise au cours du projet de recherche MOZAIC (Measurement of Ozone and Water Vapour on Airbus in-service Aircraft), qui a été financé par l'Europe entre 1993 et 2004 et CARIBIC (Civil Aircraft for the Regular investigation of the Atmosphere Based on an Instrument Container). IAGOS qui a été initiée par le centre de recherches de Jülich (DE), le CNRS, Météo-France et Airbus, produit des données pour la communauté scientifique et les opérateurs publics, avec en particulier la fourniture de données en temps réel pour les prévisions de qualité de l'air et météorologiques. Ces données sont exploitées par les réseaux scientifiques internationaux, les centres internationaux de prévisions météorologiques, les centres de prévision de la qualité de l'air, le Service Atmosphérique du programme Copernicus et plus largement par la sphère de GEOSS (Global Earth Observation System of Systems).

\section{IMPLICATIONSSOCIO-ÉCONOMIQUES}

Les partenaires historiques et fondateurs du programme sont Airbus et Météo-France. En France, la production industrielle de l'équipement (Package 1, mesurant ozone et $\mathrm{CO}$ ), des dispositifs de transmission des données en temps réel (RTTU), et des kits d'intégration sont sous-traitées à des entreprises régionales : LGM à Blagnac, Atmosphère à Toulouse, et Sabena Technics à Bordeaux.

\section{DONNÉES}

Estimation du flux de données : Environ 25 To/an.

Stockage : pris en charge par l'Observatoire Midi-Pyrénées

(Université de Toulouse en ce qui concerne la base de données centrale, les transmissions et le réseau de calcul.

Accessibilité : www.iagos.fr

Présence dans des réseaux de données : WMO (GAW), Copernicus Atmosphere Monitoring Service (CAMS)

\section{Coût de construction \\ 20 M€ au total, dont $28 \%$ pour la France Coût de fonctionnement : 0,185 M€ en 2016}

\section{Dimension internationale \\ IAGOS ESFRI Landmark \\ Coordinateur : Andreas Petzold, DE \\ Pays partenaires : DE, FR, UK \\ Site internet : www.iagos.org}

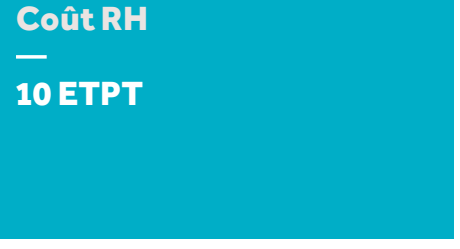

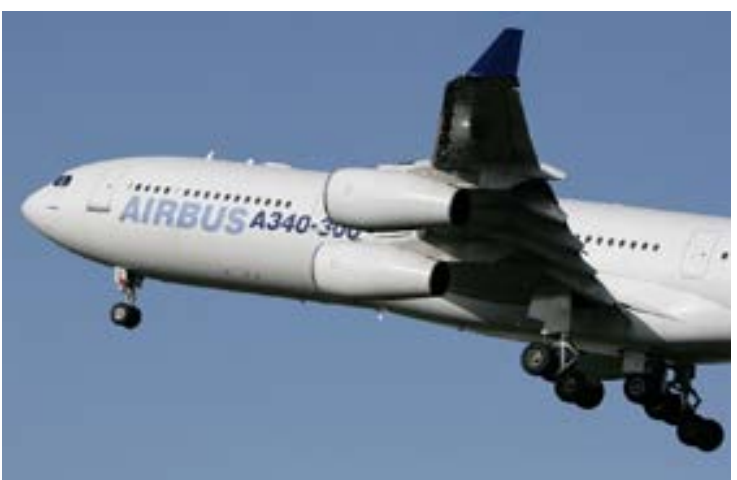

Type de l'infrastructure : IR

Statut de l'infrastructure :

AISBL

\section{Localisation :}

Toulouse (coordination)

Localisation des autres sites :

IR distribuée

Responsable de l'infrastructure: Valérie THOURET

\begin{tabular}{|r|c|}
\hline Création : & Exploitation : \\
\hline 2011 & 2011 \\
\hline
\end{tabular}

\section{Jouvence :}

Finalisation de l'équipement de 20 avions prévue pour 2025

\section{Tutelles / Partenaires :}

CNRS, Météo-France

\section{Contact en France :}

valerie.thouret@aero.obs-mip.fr valerie.thouret@iagos.org

\section{www.iagos.fr}




\section{Infrastructure LIttorale et COtière}

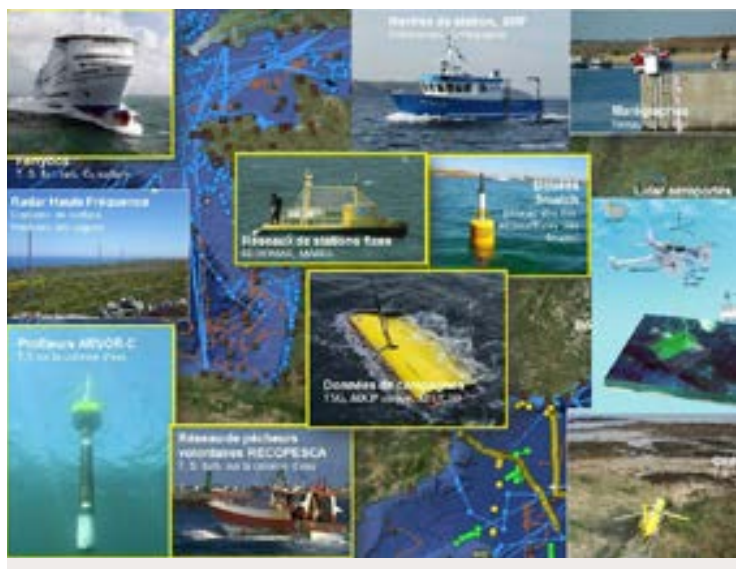

\section{Type de l'infrastructure : IR}

\section{Localisation :}

Brest (coordination)

\section{Localisation des autres sites:}

IR distribuée

\section{Responsable de l'infrastructure :} Jérôme PAILLET

\begin{tabular}{r|c} 
Création : & Exploitation : \\
\hline 2016 & 2016 \\
\hline
\end{tabular}

Tutelles / Partenaires : CNRS, IFREMER, IRD, SHOM/

Réseau des universités marines

\section{Contact en France :}

jerome.paillet@ifremer.fr
L'océan côtier et les zones littorales sontle siège de transferts et d'échanges entre différents milieux ainsi que de transformations, notamment aux travers des interactions minéral-vivant, et d'évolution des écosystèmes littoraux et côtiers. La compréhension des processus physiques, biogéochimiques et sédimentaires associés (nature, échelles de temps, liens entre eux) est fondamentale. L'observation des écosystèmes côtiers et littoraux revêt donc une ambition pluridisciplinaire. I-LICO est un ensemble de dispositifs d'observation permettant de collecter des échantillons et de déployer des capteurs afin de caractériser les évolutions des environnements côtiers et littoraux et d'avoir un suivi étendu des évolutions à long terme. Ce suivi favorisera également l'anticipation et la compréhension de certains processus et permettra de quantifier l'impact d'évènements intermittents et/ou extrêmes.

Cette infrastructure rassemble des dispositifs individuels des organismes de recherche impliqués ainsi que des SOERE (Services d'observation et d'expériementation pour la Recherche en Environnement) labélisés par AllEnvi. Elle a pour missions de:

1. veiller à ce que les observations dans les milieux littoraux et côtiers répondent aux enjeux sociétaux et questions scientifiques associées

2. fédérer et animer le réseau des observatoires des milieux littoraux et côtiers en favorisant l'interdisciplinarité:

3. être garant de l'interopérabilité et de la qualité des observations effectuées par les différents systèmes d'observation.

\section{IMPLICATIONSSOCIO-ÉCONOMIQUES}

Il existe un fort intérêt des collectivités, services décentralisés de l'état et de nombreux partenaires locaux pour de telles observations au niveau régional concernant l'état de santé des écosystèmes et récifs coralliens en Outre-Mer, la qualité des eaux, les risques littoraux, les évolutions climatiques à l'échelle régionale.

\section{DONNÉES}

Estimation du flux de données : Les flux de données seront évalués au cours des premières années.

Stockage : La bancarisation des données et services sera assurée en interface avec le pôle de données et de services OCEAN (cf. IR en projet Pôle de données.

Accessibilité : Les données seront interopérables et en libre accès pour la communauté scientifique au travers des portails des différents SNO et du pôle de données Océan.

Présence dans des réseaux de données : CORIOLIS, SEDOO, Bases de données nationales : CYBER, PELAGOS, BENTHOS, SEADATANET, COPERNICUS, CMEMS, EMODNET

\section{Coût RH}

\section{$90 \mathrm{ETPT}$}




\section{Observatoire de la Zone Critique, Applications, Recherche}

OZCAR est une infrastructure de recherche distribuée mettant en réseau des sites déployés sur le terrain, instrumentant sol, sous-sol, eau et glace pour mesurer en continu, modéliser et gérer les cycles de l'eau, du carbone et des éléments associés. Les grands enjeux scientifiques concernent une meilleure compréhension des stocks et des flux d'énergie et de matière à la surface des continents le long de gradients climatiques, topographiques, géologiques ou d'utilisation des terres.

OZCAR est une IR essentielle pour obtenir une vision élargie et consolidée des changements environnementaux à l'œuvre sur les surfaces continentales. Elle associe les observatoires de recherche élémentaires des organismes concernés et des Systèmes d'Observation et d'Expérimentation pour la Recherche en Environnement (SOERE) labélisés par AllEnvi. Elle offre une gouvernance et une méthodologie partagées au service de l'étude du fonctionnement et de l'évolution de la Zone Critique des surfaces continentales pour la recherche, l'appui aux politiques publiques et le monde économique.

Les services concernent:

- les bassins versants;

- I'hydrométéorologie;

- les aquifères:

- les sols naturels et anthropisés (agrosystèmes, friches industrielles, villes);

- la dynamique littorale

- les zones humides:

- les zones enneigées et englacées.

IMPLICATIONSSOCIO-ÉCONOMIQUES

OZCAR interagit avec les acteurs (services publics, pôles de compétitivité, entreprises...) concernés par la qualité et la gestion de l'eau, le risque hydrologique, la qualité des sols et des services écosystémiques associés, la réhabilitation des milieux, le stockage en sous-sol... Des technologies innovantes seront développées: géophysique non invasive, mesures in-situ haute fréquence, capteurs, transmission, stockage et traitement de l'information...

\section{DONNÉES}

Estimation du flux de données : Plusieurs To/an

Accessibilité : Pôle de données et de services THEIA (cf. IR en projet Pôle de données)

Présence dans des réseaux de données : Projet européen «e-LTER».

\section{Coût de construction}

Environ $10 \mathrm{M€}$

\section{Coût RH 130 ETPT}

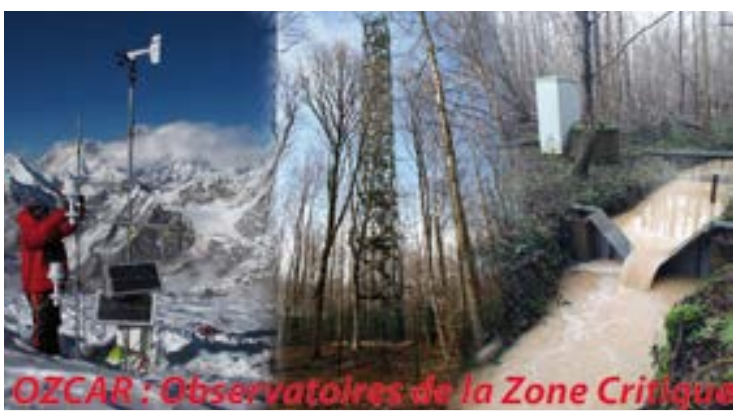

Type de l'infrastructure : IR

\section{Localisation :}

Paris (coordination)

\section{Localisation des autres sites :}

IR distribuée

\section{Responsable de l'infrastructure : Jérôme GAILLARDET}

\section{Création: Exploitation: \\ 2016 2016}

\section{Tutelles / Partenaires :}

CNRS, INRA, IRSTEA, IRD, BRGM, ANDRA IPGP, universités Aix-Marseille, Antilles, Avignon, Brest, Caen-Rouen, Franche-Comté, Grenoble, La Réunion, Orléans, Poitiers, Montpellier, Paris-Est Créteil, Paris-UPMC, Paris-Sud, Nancy, Rennes, Strasbourg, Toulouse

\section{Contact en France :}

gaillardet@ipgp.fr

\author{
Dimension internationale \\ Projet d'infrastructure de recherche ESFRI « eLTER » dans la continuité \\ du projet INFRAIA « eLTER» \\ Coordinateur: I. Kühn, M. Mirtl, DE \\ Pays partenaires : DE, AT, BG, BE, CH, CZ, ES, FI, GR, HU, IL, IT, NL, PT, RO, \\ RS, SE, SK, SI, UK \\ Site internet: $\underline{w w w . I t e r n e t . e d u}$
}




\section{Ressources Agronomiques pour la Recherche}

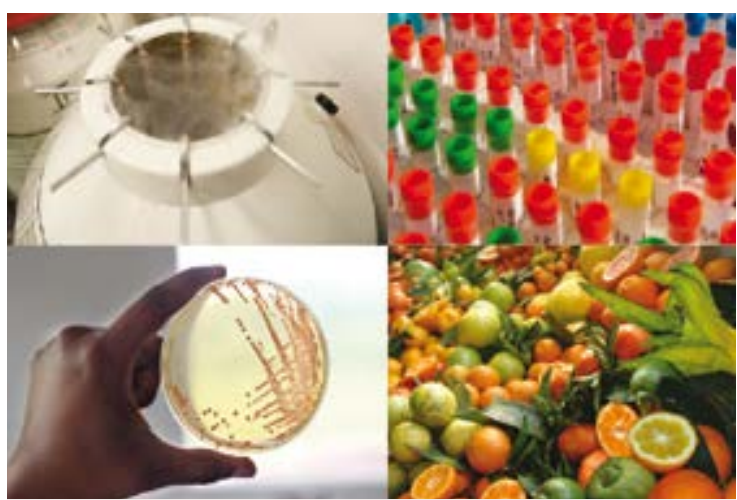

Type de I'infrastructure : IR

\author{
Localisation : \\ Paris (coordination) \\ Localisation des autres sites : \\ IR distribuée
}

Responsable de l'infrastructure:

Michèle TIXIER-BOICHARD

Création: Exploitation:

2015

2016

Tutelles / Partenaires:

INRA, CIRAD, CNRS, IRD

\section{Contact en France :}

contact@rare.eu

\author{
animal : \\ www.crb-anim.fr/Le-CRB-Anim \\ végétal et microbien : \\ www.ibisa.net/
}

RARe a pour objectifs d'améliorer la gestion et visibilité nationale des ressources biologiques hébergées par les Centres de Ressources Biologiques (CRB) qui la constituent et de faciliter leur utilisation par un grand nombre de chercheurs de la communauté agronomique comme d'autres, au niveau national et à terme européen où il n'existe pas, excepté le centre de ressources du WUR (Wageningen, NL), d'organisation identique.

La capacité à maintenir une grande diversité de ressources documentées, à en collecter de nouvelles, à contribuer à leur caractérisation, à les distribuer et à gérer des données associées, place les CRB de RARe au cœur de nombreux programmes de recherche destinés à explorer le vivant et les écosystèmes ainsi qu'à valoriser la biodiversité pour l'agriculture et l'industrie, l'alimentation, l'environnement et la santé.

La valeur ajoutée de RARe consiste à mutualiser les compétences, harmoniser les pratiques, susciter des projets de biologie comparée et proposer un portail d'entrée unique pour faciliter l'accès à des échantillons, en tenant compte du contexte règlementaire qui varie avec la nature biologique des ressources, pour les aspects sanitaires comme pour les aspects juridiques, et avec les politiques partenariales des organismes de recherche.

RARe apportera un soutien organisationnel à ses membres dans l'application du protocole de Nagoya et de la loi française sur la biodiversité. L'infrastructure connexe «CRB animal » du domaine Biologie et Santé, concerne en plus les ressources biologiques hors champ agronomique.

\section{IMPLICATIONSSOCIO-ÉCONOMIQUES}

Tous les secteurs de l'agriculture sont concernés : productions végétales, animales, industries agro-alimentaires, valorisation non alimentaire de la biomasse, biotechnologie des clean tech. Chaque sous-ensemble de CRB constitutif de RARe a déjà un réseau établi de partenaires, issus du secteur agricole mais aussi du secteur du diagnostic et de la pharmacie, du secteur des biotechnologies et du secteur de la cryobiologie. Le partenariat avec la recherche pour le développement est constitutif de certains CRB.

\section{DONNÉES}

Estimation du flux de données : 10 To en 2015.

Stockage : Local, au sein des composantes de RARe.

Accessibilité : L'accès est libre, après acceptation d'une charte des utilisateurs, pour les données passeport de qualité technique, reproductive et sanitaire des échantillons conservés. Il est restreint pour les résultats de caractérisation avancée.

Présence dans des réseaux de données : DDBJ/ENA/GenBank (caractérisation génomique),ressources phytogénétiques : (i) standards internationaux, Multicrop Passport Data, descripteurs ECPGR ou IPGRI, standards taxonomiques (ii) réseau international de la Crop Ontology (iii) projets internationaux (WheatIS, DivSeek, Elixir-Excelerate)

\author{
Dimension internationale \\ MIRRI (Microbial Resource Research Infrastructure)), ESFRI Roadmap \\ Coordinateur : Erko Stackebrandt, DE \\ Pays partenaires : UK, NL, ES, PL, PT, IT, BE, SE, RU, FR \\ Site internet : MIRRI www.mirri.org
}




\section{Réseau des Collections Naturalistes françaises}

Les collections publiques en France conservent plus de 100 millions de spécimens. RECOLNAT propose la valorisation de 350 ans d'herbiers, d'animaux naturalisés et de fossiles. Cette source de données est aujourd'hui revisitée à la faveur du débat sur le changement global. Actuellement, plus de 9 millions d'objets sont en cours de numérisation.

Les collections constituent à la fois un patrimoine scientifique, une archive de la biodiversité et une infrastructure de recherche. L'accès aux spécimens a permis de fonder les concepts des espèces actuelles et fossiles. De même, leur informatisation est essentielle pour la recherche en taxinomie.

Les collections taxinomiques françaises figurent parmi les premières d'Europe, mais beaucoup sont sous-utilisées par les chercheurs, car peu accessibles. Par la mise en place de services communs, RECOLNAT en facilite l'accès et assure de meilleures conditions d'études. L'observation des images à distance participera à une nouvelle façon de travailler par le biais d'un laboratoire virtuel, les chercheurs pourront ensuite venir consulter directement les spécimens qu'ils auront repérés.

RECOLNAT dont les collections informent sur la répartition des espèces dans l'espace et le temps contribuera à l'expertise environnementale, aux questions de santé et de sécurité alimentaire. L'information est accessible à tous et chacun peut participer à son élaboration. Les amateurs constituent des réseaux de compétences et d'expertise. Un site de science participative propose déjà aux citoyens de compléter les étiquettes d'herbier à partir des images.

\section{IMPLICATIONS SOCIO-ÉCONOMIQUES}

Numérisation industrielle par Picturae. Restauration d'herbiers mise au point par GRAHAL.

Agoralogie, CNAM et MNHN : site de science citoyenne Les Herbonautes totalise 1,5 millions de contributions. Grâce au GBIF (Global Biodiversity Information Facility), le site est cloné au Portugal et en Allemagne.

Fournisseurs de données : plus de 70 partenaires, la liste régulièrement actualisée est disponible sur la page recolnat.org/ip/liste.html

\section{DONNÉES}

Stockage : plusieurs To

Accessibilité : Accès libre et gratuit pour des images à l'unité. Sous forme de services facturés en cas de consultations multiples.

Présence dans des réseaux de données : ECOSCOPE , INPN, GBIF

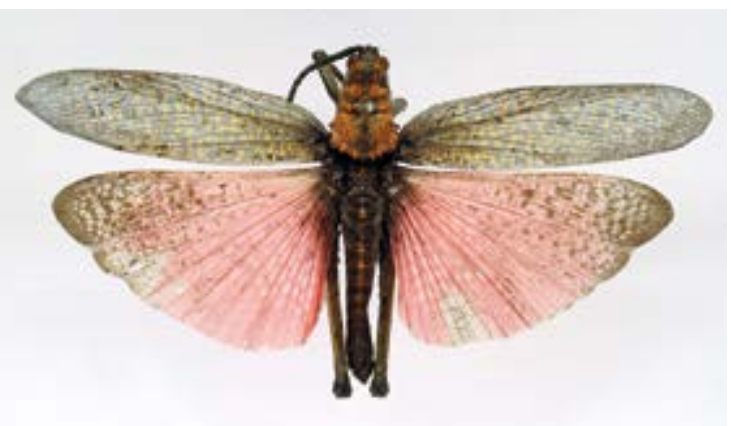

Type de l'infrastructure : IR

\section{Localisation:}

Paris (coordination)

Localisation des autres sites :

IR distribuée

Responsable de l'infrastructure :

Marc PIGNAC

\begin{tabular}{|r|c|}
\hline Création : & Exploitation : \\
\hline 2013 & 2018 \\
\hline
\end{tabular}

\section{Tutelles / Partenaires :}

MNHN, Univ. Montpellier, Univ. Bourgogne, Univ. Clermont-Ferrand, IRD, CNAM, INRA,

Tela Botanica, Agoralogie

\section{Contact en France :}

contact@recolnat.org

\section{recolnat.org}




\section{Réseau sismologique et géodésique français/ European Plate Observing System}

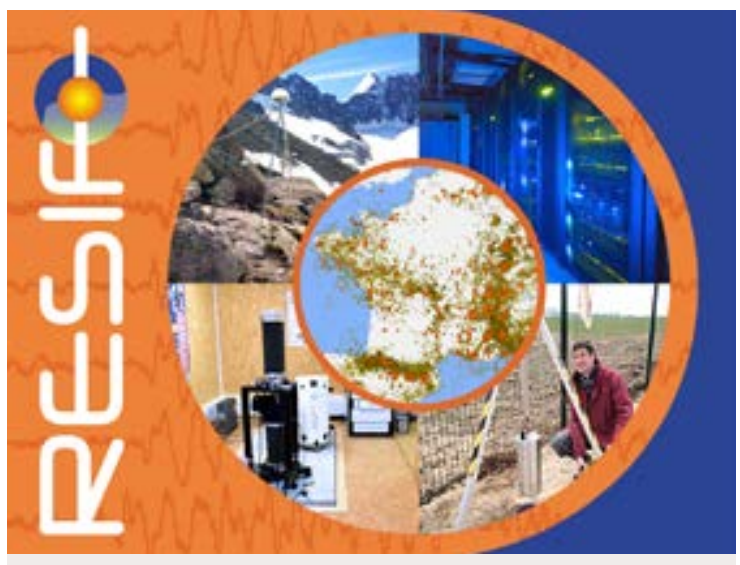

Type de l'infrastructure : IR

\section{Localisation :}

Paris (coordination)

\section{Localisation des autres sites :}

IR distribuée

\section{Responsable de l'infrastructure : \\ Stéphane MAZZOTTI}

\section{Création :}

2011

\section{Tutelles / Partenaires :}

CNRS, BRGM, CEA, CNES, IFREMER, IFSTTAR, IGN, IPGP, IRD, IRSN, OCA, Univ. Clermont Ferrand, Univ. Grenoble, Univ. Montpellier, Univ. Strasbourg, Univ. Nantes, Univ. Nice Sophia Antipolis, Univ. Toulouse

\section{Contact en France : contact@resif.fr}

\section{wWw.resif.fr}

RESIF/EPOS a comme objectif de doter la France d'une instrumentation moderne pour comprendre la dynamique de la Terre. RESIF/EPOS fournira les données permettant d'étudier les séismes en France et la propagation des ondes sismiques dans le sous-sol, avec des instruments (sismomètres, antennes GNSS et gravimètres) permettant de mesurer la déformation de la surface terrestre depuis les mouvements tectoniques lents jusqu'aux secousses sismiques instantanées. L'installation de nombreux instruments couvrant toute la France métropolitaine ouvre une nouvelle fenêtre vers l'intérieur de la terre, afin de mieux comprendre son fonctionnement et sa dynamique, depuis son noyau jusqu'à l'interaction avec ses enveloppes fluides (atmosphère et hydrosphère).

RESIF/EPOS permettra également de mieux comprendre les aléas telluriques en France. Les séismes majeurs sont peu fréquents en France métropolitaine, mais leur impact socio-économique est potentiellement tel que la prévention et la gestion de la vulnérabilité face au risque sismique est devenue une préoccupation centrale. En fédérant les expertises en matière de méthodes innovantes d'imagerie sismique et de géodésie, RESIF/EPOS fournira également des données clefs pour mieux comprendre le sous-sol, afin d'en permettre une gestion optimisée et modérée. Toutes les données provenant des instruments RESIF/EPOS sont distribuées librement et gratuitement.

\section{IMPLICATIONS SOCIO-ÉCONOMIQUES}

RESIF/EPOS fournit la donnée d'entrée pour des produits dérivés, créés par les membres du Consortium RESIF/EPOS. Ces données constituent des éléments clés dans la prise de décisions importantes associés à l'aléa tellurique et à la gestion du sous-sol français. RESIF/EPOS travaille par ailleurs en étroite coopération avec une PME française qui développe un nouveau type de gravimètre portable.

\section{DONNÉES}

Estimation du flux de données : $20 \mathrm{To} / \mathrm{an}$

Accessibilité : Pôle de données et de services Form@Terre (cf. IR en projet Pôle de données) ; Données disponibles sur le site (www.resif.fr). Accès libre et gratuit.

Présence dans des réseaux de données : FDSN, EIDA, BGI, GGP.

\section{Coût de construction \\ $10 \mathrm{M€}$ \\ Coût de fonctionnement : $1,1 \mathrm{M} €$}

\author{
Dimension internationale \\ EPOS, ESFRI Roadmap \\ Coordinateur : Massimo Cocco, IT \\ Pays partenaires : CZ, DK, FR, DE, GR, IS, IE, IT, NL, NO, PL, PT, RO, ES, \\ $\mathrm{SE}, \mathrm{CH}, \mathrm{TR}$, UK \\ Site internet : $\underline{w w w . e p o s-e u . o r g}$
}




\section{Service des Avions Français Instrumentés pour la Recherche en Environnement}

SAFIRE opère 3 avions de recherche (ATR 42, Falcon 20 et Piper Aztec) couvrant des domaines de vols différents pour réaliser des campagnes de mesures scientifiques dans les domaines suivants : physique et chimie de l'atmosphère, surfaces continentales, surfaces océaniques, recherche et technologie dans l'aérospatial. Les objectifs scientifiques, en lien avec les équipes scientifiques extérieures sont de

- acquérir des données à différentes altitudes lors de campagnes coordonnées d'observations pour soutenir les progrès de la compréhension des processus expliquant le fonctionnement de l'environnement;

- conduire les campagnes de calibration/validation des nouveaux instruments embarqués sur satellite et des concepts d'instruments préfigurant de futures missions spatiales.

Les 3 avions possèdent de nombreuses capacités d'emports spécifiques pour de nombreux équipements et instruments. SAFIRE met au service des communautés son savoir-faire pour installer différents équipements et instruments à bord puis préparer et réaliser des vols de mesures, partout dans le monde, suivant chaque besoin.

\section{IMPLICATIONSSOCIO-ÉCONOMIQUES}

La coopération avec des industriels français de l'aéronautique (PME et grands groupes) et l'appui au domaine spatial (observation de la Terre, calibration/validation d'instruments embarqués sur satellite) permet à SAFIRE de renforcer ces secteurs d'activités au niveau mondial. SAFIRE pourra également jouer un rôle dans le développement de pôles régionaux sur des activités comme l'utilisation des drones.

\section{DONNÉES}

Estimation du flux de données : Environ 2 Go de données par an

Stockage : Environ 7 To de données

Accessibilité : Pôle de données et de services AERIS (cf. IR en projet Pôle de données). Accès libre et gratuit pour des images à l'unité. Sous forme de services facturés en cas de consultations multiples.

Présence dans des réseaux de données : Pôle de données et de services AERIS (cf. IR en projet Pôle de données).

\section{Coût de construction \\ $25 \mathrm{M€}$ \\ Coût de fonctionnement : 1,7 M€}

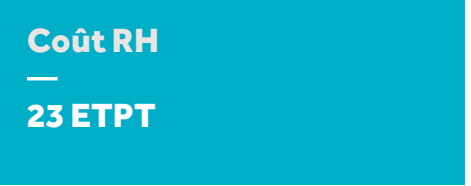

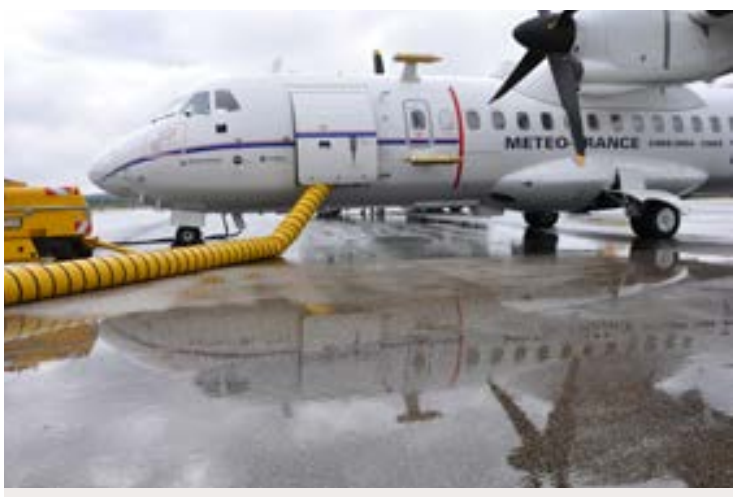

Type de I'infrastructure : IR

Statut de l'infrastructure :

Unité mixte de service

\section{Localisation:}

Cugnaux (coordination)

Responsable de l'infrastructure :

Aurélien BOURDON

\begin{tabular}{|r|c|} 
Création : & Exploitation : \\
\hline 2005 & 2006 \\
\hline
\end{tabular}

\section{Jouvence :}

Achat et équipement d'un nouvel avion dans la période 2018-2020

\section{Tutelles / Partenaires :}

CNES, CNRS, Météo-France

\section{Contact en France :}

desk@safire.fr

www.safire.fr

\section{Dimension internationale}

EUFAR ( European Fleet for Airborne Research)

Coordinateur : Phil Brown, UK, Metoffice

Pays partenaires: DE, CZ, IT, FR, ES, UK, AT, IL, CH, PL, BE

Site internet : www.eufar.net 


\section{Pôle de données et services pour le Système Terre}

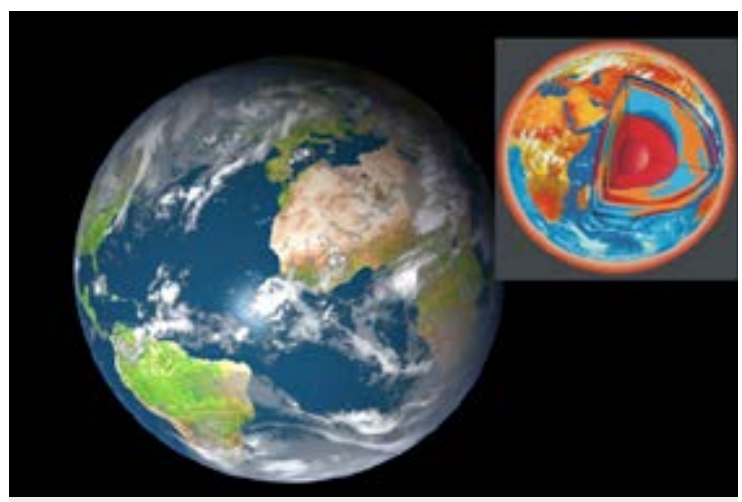

Type de l'infrastructure : Projet

\section{Localisation : \\ Paris (coordination) \\ Localisation des autres sites : \\ IR Virtuelle}

\section{Responsables de l'infrastructure :}

Nicole Papineau (AERIS), Michel Diament (Form@Terre), Nicolas Baghdadi (THEIA), Fabienne Gaillard (OCEAN)

\section{Création : \\ Exploitation : \\ 2016 2017}

\section{Tutelles / Partenaires :}

CNES, CNRS, Ifremer, IRD, IRSTEA,

Météo France, SHOM, BRGM, CEA, CEREMA,

CIRAD, IGN, INERIS, INRA, IPGP, ONERA, OCA,

Observatoire de Paris, Ecole Polytechnique,

Réseau des Universités marines, Univ. Lille 1, Univ. Toulouse, Univ. Pierre et Marie Curie/ Région Nord-Pas-de-Calais

\section{Contact en France :}

nicole.papineau@ipsl.jussieu.fr diament@ipgp.fr

nicolas.baghdadi@teledetection.fr

fabienne.gaillard@ifremer.fr
Pour compléter le dispostif des infrastructures de recherche (IR) d'observation du Système Terre et organiser de manière intégrée l'accès aux données, l'infrastructure de recherche se propose de mettre à disposition les données, les produits et des services relatifs à l'observation de ce système, via les pôles de données et de services existants: AERIS (atmosphère), Form@Terre (Terre Profonde), THEIA (surfaces continentales) et OCEAN (Océans et Littoral). Elle donnera accès aux données produites par les IR d'observation, les infrastructures dites "logistiques" (bâteaux, avions, ballons...) ainsi que les données des satellites.

Destinés en priorité à la communauté scientifique française, ces produits ont aussi une portée internationale (missions satellites, réseaux d'observation globaux, partenariats pour le développement).

Afin de garantir le respect des standards scientifiques et techniques en vigueur, les produits de I'IR sont définis et élaborés sous la responsabilité d'experts. Les séries de données et produits issus des réseaux d'observation, des campagnes et chantiers nationaux, des missions satellites, seront qualifiées, décrites et interopérables. À partir de ces données, I'IR élaborera ses propres produits.

Pour faciliter l'exploitation des informations I'IR mettra à disposition des outils d'exploration, de visualisation, d'extraction et d'analyse ainsi que les moyens de calculs appropriés.

L'IR hébergera des plateformes collaboratives pour le partage d'information et de bonnes pratiques et participera à la formation scientifique et technique des communautés concernées.

\section{IMPLICATIONSSOCIO-ÉCONOMIQUES}

Les données fournies par I'IR aident à la mise en œuvre des politiques publiques car les travaux utilisant ces données ont un impact socio-économique dans les domaines des risques naturels, du changement climatique, des ressources minérales et en eau.

Au côté des centres de données implantés en région se développent des pépinières de PME spécialisées dans le traitement et l'utilisation des données.

\section{DONNÉES}

Estimation du flux de données : Jusqu'à plusieurs Po/an

Stockage : Centres de données de l'IR

Accessibilité : Web, FTP

Présence dans des réseaux de données : RESIF/EPOS, EURO-ARGO, EMSO, ICOS, IAGOS, ACTRIS, EUFAR, EmodNet, Copernicus et plusieurs réseaux mondiaux contributeurs à GEOSS 
SCIENCES DU SYSTÈME TERRE ET DE L'ENVIRONNEMENT 

ÉNERGIE

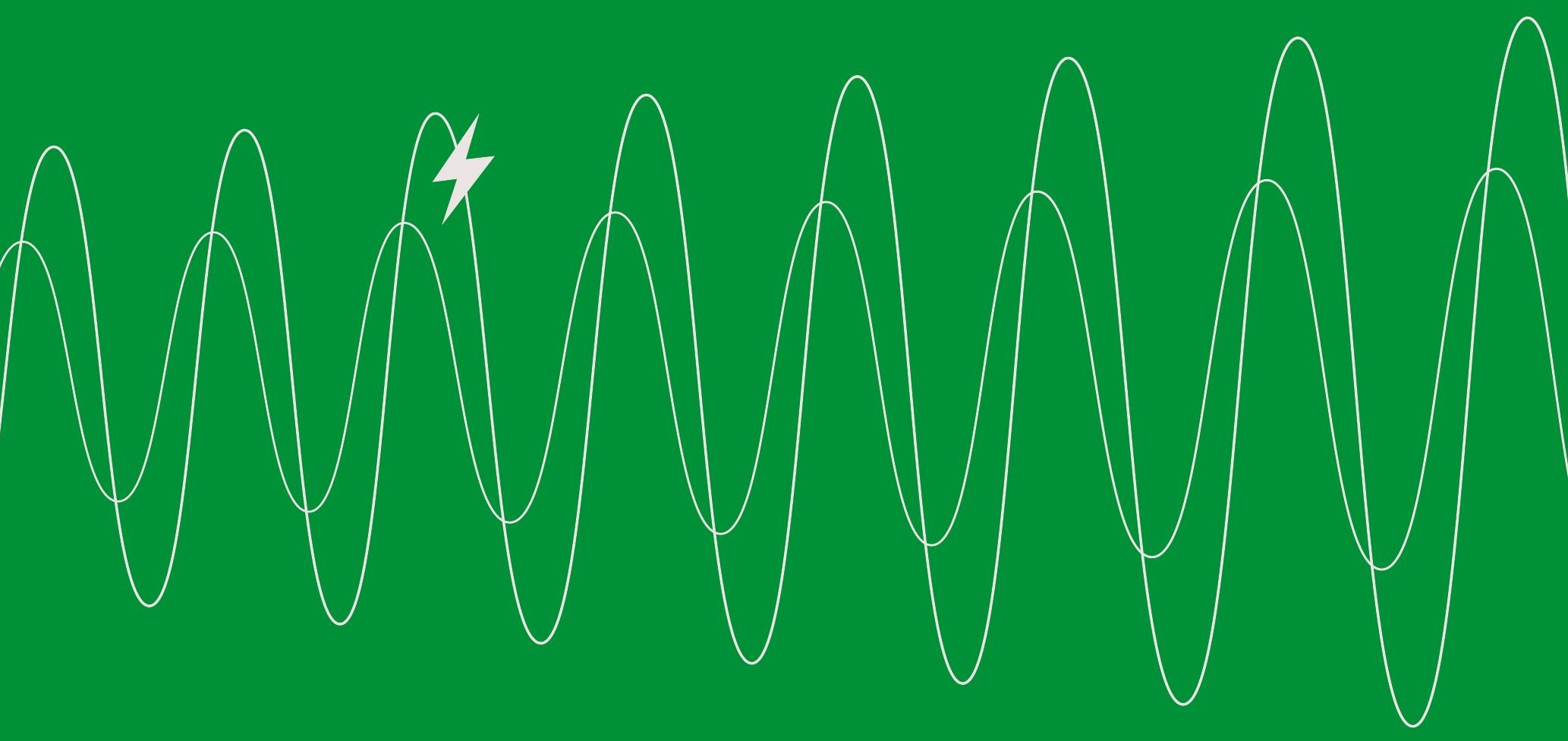




\section{Énergie}

\section{CONTEXTE GLOBAL}

Les perspectives d'un développement durable et économiquement acceptable sont totalement dépendantes d'un approvisionnement en énergie pérenne, sécurisé avec un impact sur l'environnement réduit, tant à l'échelle locale que globale. La problématique énergétique s'inscrit dans un cadre complexe qui doit répondre à 4 défis majeurs : garantir l'accès à l'énergie des populations, éviter la précarité énergétique, s'inscrire dans le contrôle du réchauffement climatique, offrir un mix énergétique durable.

Ainsi, le monde de l'énergie doit satisfaire l'accroissement de la demande(entre 2000 et 2030 , une croissance d'environ $60 \%$ de la demande est attendue) par une diversification large des sources d'énergie, par l'augmentation de la production d'une énergie défossilisée accompagnée de la pénétration des énergies variables, notamment pour contrôler les rejets de gaz à effet de serre. Pour répondre aux besoins de diversification d'approvisionnement et aux grands enjeux climatiques, le xxle siècle sera donc celui de la transition énergétique et la France s'est notamment dotée en 2015 d'une « loi pour la transition énergétique et la croissance verte .

La maîtrise du mix énergétique à l'horizon 2050 est donc un défi majeur d'envergure internationale qui conduit à de très gros besoins en recherche et en équipements spécifiques.

\section{LES GRANDS AXES DE PROGRĖS ET LES GRANDES INFRASTRUCTURES ASSOCIÉES}

Le futur mix énergétique mondial nécessite de déployer le plus rapidement possible des solutions alternatives: la biomasse, la valorisation des déchets, l'énergie solaire photovoltaïque ou thermodynamique, l'énergie éolienne terrestre ou offshore, les énergies marines, notamment hydroliennes, l'hydraulique, la géothermie, mais aussi de développer le nucléaire de nouvelle génération et les techniques de réduction de l'empreinte carbone de la production d'électricité thermique par les techniques de Capture, transport stockage et valorisation du $\mathrm{CO}_{2}$ (CCSV). Un important gisement existe mais quelle qu'en soit la forme, ces solutions requièrent d'importants investissements, d'importants travaux de R\&D avec un fort besoin en infrastructures de recherche dédiées. En collaboration avec l'Alliance ANCRE, un travail d'analyse du gisement français d'infrastructures a été coordonné par la DGRI et a conduit à proposer cinq (5) d'entre elles comme les plus représentatives des besoins à venir. A ce jour, ces infrastructures ont des niveaux de maturité et d'organisations variables qui les conduisent à disposer soit d'un statut d'Infrastructure, soit d'un statut de projet.

Le déploiement à grande échelle des énergies renouvelables variables demande d'avoir recours à de nouvelles installations plus performantes et/ou plus compétitives qui ne sont pas encore disponibles ou seulement au stade de prototypes. Un effort de recherche important, basé sur des moyens expérimentaux de pointe allant jusqu'à l'échelle pilote est donc nécessaire.

Les axes qui sont privilégiés pour les années à venir porteront sur:

- le solaire, avec:

- FR SOLARIS, nœud français de l'infrastructure EU SOLARIS «European SOLAR Research Infrastructure for Concentrating Solar power " - inscrite sur la feuille de route ESFRI depuis 2010, qui s'appuie sur les installations solaires d'Odeillo/Thémis pour le solaire thermodynamique ;

- SOPHIRA qui est introduit sur la feuille de route nationale des infrastructures - Edition 2016, comme " projet ", et qui offrira un potentiel expérimental de premier plan pour le solaire photovoltaïque en s'appuyant sur les acquis du projet européen SOPHIA et sur le potentiel de l'Institut National de I'Energie Solaire (INES);

- les énergies marines, avec:

- THEOREM qui étudie les énergies des mers (courants, marées, houle, énergie thermique) dont le potentiel est très important et encore peu exploité. Cette source d'énergie est, en outre, difficile à domestiquer. A moyen terme, les axes dedéveloppement seront vraisemblablement sur l'énergie hydrolienne et les marées et à plus long terme, sur l'énergie houlomotrice et l'énergie thermique. Aujourd'hui, peu d'équipements sont disponibles pour accompagner ces développements. THEOREM offrira ce potentiel. Il s'appuiera sur les équipements nationaux de l'Ecole Centrale de Nantes et de l'Ifremer. THEOREM apparaîtra en tant que projet sur la feuille de route nationale des infrastructures - Edition 2016. 
L'énergie nucléaire contribuera significativement au mix électrique français dans un avenir court - moyen - long terme, avec les réacteurs de troisième génération (EPR) en construction et ceux de quatrième génération attendus à I'horizon 2040-2050. Des recherches plus amont pour le développement de la fusion nucléaire sont également en cours et la réalisation d'un démonstrateur technologique (DEMO après ITER) demandera encore plusieurs décennies. Offrir les moyens expérimentaux conduisant à améliorer les connaissances dans le domaine de la fusion nucléaire est d'une grande importance. De grandes infrastructures s'inscrivent dans ce besoin et sont fortement engagées dans des consortiums de recherche internationaux. Dans le cadre de cette feuille de route, il s'agit de :

- WEST (Tungsten (W) Environment in Steady-state qui a pour fonction de faire évoluer le tokamak Tore Supra (construit et opéré sous l'égide de l'Association EURATOM-CEA dans les années 80 ) en soutien direct à la construction d'ITER.

Concernant ITER, bien que géré dans des cadres spécifiques, il doit être mentionné pour son importance. En effet, il doit démontrer la maîtrise de l'énergie de fusion par confinement magnétique et permettre le développement à terme d'une nouvelle source énergétique et accompagner l'application industrielle. Dans le cadre d'une coopération internationale, ITER est en cours de construction en France, sur le site de Cadarache et l'Europe contribue à une large part du projet. De plus, il existe un programme de recherche Euratom Fusion dans le cadre du programme Horizon 2020, destiné à coordonner les activités de recherche des états membres, dont un volet concernant les matériaux et DEMO, l'étape qui suivra ITER.

Enfin, la contribution à la production d'électricité via des ressources fossiles accompagnera la aussi la transition énergétique et écologique. Toutefois, le passage par le captage, le transport, le stockage et la valorisation du $\mathrm{CO}_{2}$ sera incontournable. Une infrastructure est dédiée à cette problématique. Il s'agit de :

- ECCSEL (European Carbon dioxyde Capture and Storage Laboratory) qui est un projet inscrit sur la feuille de route ESFRI depuis 2008. C'est une infrastructure distribuée avec le BRGM comme nœud français et pilote. ECSSEL apparaît en tant que projet, sur la présente feuille de route.

\section{LISTE DES INFRASTRUCTURES DE RECHERCHE DANS LE DOMAINE ÉNERGIE}

\begin{tabular}{|c|c|c|c|}
\hline CATÉGORIE & NOM & NOM COMPLET & ESFRI \\
\hline IR & FR-SOLARIS & $\begin{array}{l}\text { Infrastructure de Recherche française sur le solaire } \\
\text { thermique concentré/Solar Thermal Research } \\
\text { Infrastructure for Concentrated Solar Power }\end{array}$ & $\begin{array}{l}\text { EU-SOLARIS } \\
(2010)\end{array}$ \\
\hline IR & WEST & $\begin{array}{l}\text { W(Tungsten) Environment for Steady-state } \\
\text { Tokamaks }\end{array}$ & \\
\hline Projet & ECCSEL-FR & $\begin{array}{l}\text { European Carbon Dioxide Capture and StoragE } \\
\text { Laboratory Infrastructure }\end{array}$ & ECCSEL (2008) \\
\hline Projet & SOPHIRA & $\begin{array}{l}\text { Infrastructure pour la recherche sur le solaire } \\
\text { photovoltaïque/SOlar PHotovoltaic Research } \\
\text { Infrastructure }\end{array}$ & \\
\hline Projet & Theorem & $\begin{array}{l}\text { Réseau de Moyens d'Essais en Hydrodynamique } \\
\text { pour les Énergies Marines Renouvelables }\end{array}$ & \\
\hline
\end{tabular}



Infrastructure for Concentrated Solar Power

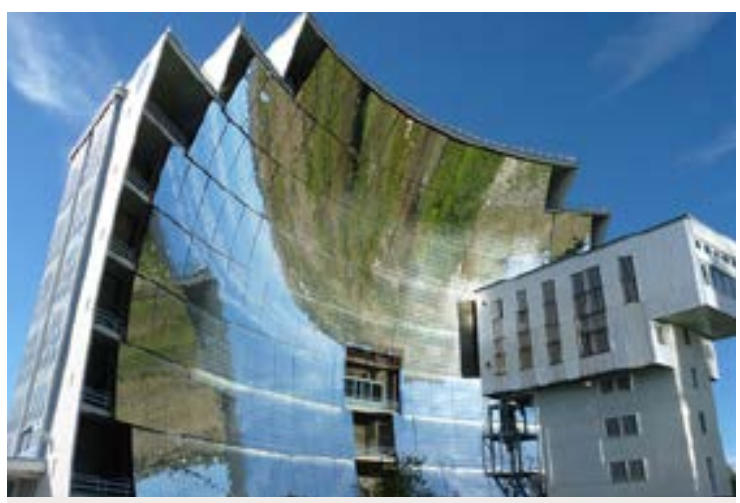

Type de l'infrastructure : IR

\section{Localisation de l'infrastructure :}

Font-Romeu, Targassone

\section{Responsable de l'infrastructure :}

Gilles FLAMANT

\section{Four solaire}

Création: Exploitation: Maintenance:

1959

1972

2015-2016

\section{Thémis}

Création:

Exploitation :

1979

1983

Microcentrale cylindro-parabolique

Création: Exploitation:

2015

2016

Tutelles / Partenaires :

CNRS

\section{Contact en France :}

Gilles.Flamant@promes.cnrs.fr

\section{www.equipex-socrate.fr}

FR Solaris a pour mission:

- de contribuer au développement des connaissances sur les thèmes suivants

- photophysique (ex: conversion photovoltaïque sous concentration);

- photochimie (ex: photocatalyse);

- thermochimie (ex: production de combustibles de synthèse);

- thermique (en particulier transferts radiatifs);

- nanomatériaux (ex: élaboration de nanopoudres);

- métallurgie (traitement de surface, frittage...);

- matériaux céramiques (applications spatiales en particulier);

- d'améliorer les technologies de conversion et stockage de l'énergie solaire thermique;

- de définir des méthodologies et procédures de qualification de composants.

L'infrastructure de recherche FR-SOLARIS est aujourd'hui constituée des

5 grandes familles d'équipements

- 1 four solaire de 1 MW (MWSF) et son environnement;

- 11 fours solaires de moyenne puissance (MSSFs) et leur environnement;

- 1 concentrateur parabolique de $50 \mathrm{~kW}$;

- 1 microcentrale solaire de $150 \mathrm{~kW}$ th et $15 \mathrm{kWel}$;

- 1 concentrateur à tour de 5 MW.

Les services proposés sont la mise à disposition de fours solaires de petite puissance (1 kW) aux équipes issues de la recherche publique, la réalisation de mesures et tests à haute température (jusqu'à $3000^{\circ} \mathrm{C}$ ), la définition et la réalisation de projets collaboratifs, I'assistance à la mise en place d'une instrumentation adaptée aux systèmes à concentration, la qualification de composants solaires, des tests de vieillissement accéléré sous irradiation solaire.

\section{IMPLICATIONSSOCIO-ÉCONOMIQUES}

Partenariats avec l'industrie française : EDF, CNIM, SNECMA, Arkema, Enogia, ADF... et avec l'industrie européenne via des projets FP7 et $\mathrm{H} 2020$.

La région Midi-Pyrénées Languedoc-Roussillon a sélectionné le thème "Solaire concentré, solaire à haut rendement " comme priorité dans le cadre de l'initiative $3 \mathrm{~S}$.

Le Conseil départemental des Pyrénéees Orientales a créé en concertation avec la région la structure de promotion du site de Thémis : Thémis Solaire Innovation (TSI).

\section{Coût de fonctionnement}

$$
0,110 \mathrm{M€}
$$

\section{Coût RH}

\section{ETPT}

\section{Dimension internationale}

EU-Solaris (the European Solar Research Infrastructure for Concentrated Solar Power), ESFRI Roadmap depuis 2010

Coordinateur: ES

Pays partenaires : ES, DE, FR, IT, EL, PT, TR

Site internet : $\underline{\text { www.eusolaris.eu }}$ 


\section{W(Tungsten) Environment for Steady-state Tokamaks}

L'objectif de WEST est la validation du design et de la fabrication du divertor d'ITER. Pour ce faire un grand nombre d'actions est nécessaire, impliquant:

- le design détaillé vérifiant le bien fondé des hypothèses ITER;

- la compréhension et maîtrise de l'interaction plasma-paroi en environnement métallique, de l'érosion/redéposition et de la rétention des combustibles du plasma; comportement des matériaux soumis à ces conditions extrêmes; mesure et interprétation des températures de surface dans des environnements 3D réfléchissants:

- I'industrialisation des composants (qualité, production zéro-défaut, coûts...);

- les modes opératoires de ce divertor dans ITER; méthodologies de mesure et de contrôle en temps réel:

- le comportement des composants dans la durée : altérations, protocoles de réparation/rénovation;

- un investissement partenarial de 24,8 M€ pour modifier la chambre plasma de Tore Supra pour ajout d'un divertor tungstène refroidi, production des plasmas et caractérisation;

- des investissements «à la carte» des partenaires, permettent d'enrichir les mesures physiques, suivant un modèle de fourniture « clé en main », incluant personnel d'exploitation scientifique et propositions expérimentales;

- les plateformes de simulation et de modélisation de I'IRFM pour la préparation, la conduite et l'interprétation des expériences;

- une plateforme d'accueil des collaborateurs et une organisation spécifique de I'IRFM favorisant leur intégration aux équipes.

\section{IMPLICATIONSSOCIO-ÉCONOMIQUES}

WEST fédère les forces scientifiques et techniques du domaine localement et régionalement (académiques, recherche, industries et éducation) du domaine, et leur offre le tremplin unique vers l'utilisation d'ITER.

Les emplois et contrats générés par la fusion sont d'importance capitale pour le développement économique du Val de Durance, et WEST irrigue dans l'ensemble du tissu industriel de la région notamment.

\section{Coût de construction}

$-$

$300 \mathrm{M€}$

Coût de fonctionnement : $15 \mathrm{M€}$

\section{Coût RH}

\section{ETPT}

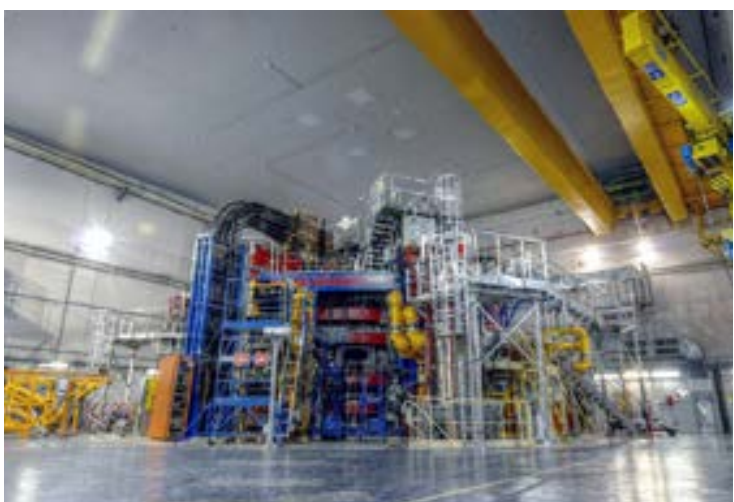

Type de l'infrastructure : IR

\section{Localisation:}

Cadarache

Responsable de l'infrastructure: Alain BÉCOULET

\section{Création: Exploitation: \\ 2013 \\ 2016}

\section{Maintenance :}

Phase $1: 2016-2018$, phase $2: 2019$

\section{Tutelles / Partenaires :}

CEA

\section{Contact en France :}

alain.becoulet@cea.fr

west.cea.fr

\section{Dimension internationale}

WEST est le nœud français dans le réseau européen (H2020/EUROfusion) et mondial (ITER agreement) des infrastructures de fusion.

Coordinateur : FR (CEA)

Pays partenaires: FR, DE, JP, IN, CN, KR, CZ, PL, UE

Site internet : west.cea.fr 


\section{European Carbon Dioxide Capture and StoragE Laboratory Infrastructure}

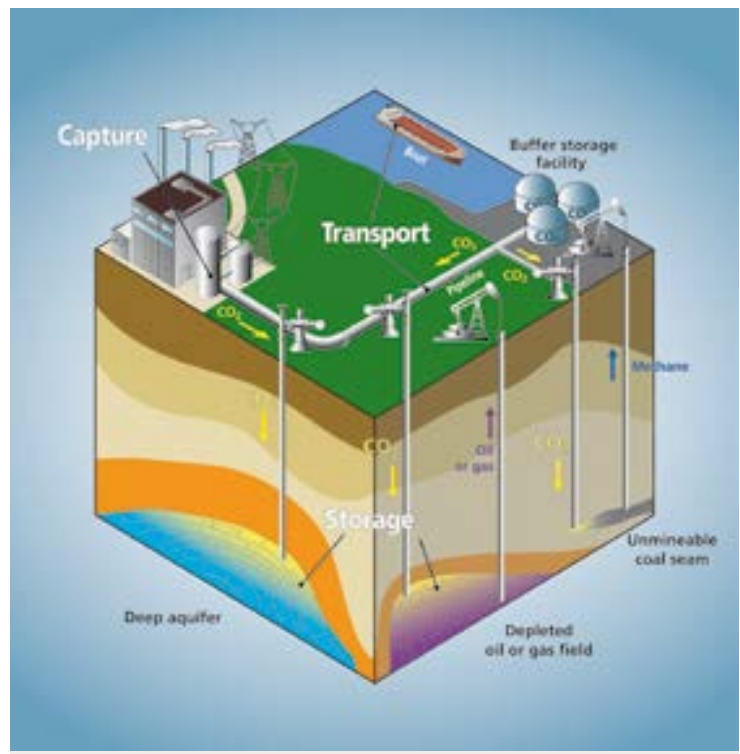

Type de l'infrastructure : Projet

\section{Localisation de l'infrastructure :}

Orléans

\section{Localisation des autres sites:}

Bure, Catenoy, Le Havre, Mont La Ville, Orléans, Rustrel, 1 équipement en projet à Lacq

\section{Responsable de l'infrastructure :} Isabelle CZERNICHOWSKI-LAURIOL

\section{Création: Exploitation: 2008 2016}

Tutelles / Partenaires :

BRGM, Andra, CNRS, EDF, INERIS, TOTAL

\section{Contact en France :}

i.czernichowski@brgm.fr

Pour accélérer le développement de la capture et du stockage du carbone et permettre sa mise en œuvre en Europe et dans le monde, il faut intensifier les efforts de R\&D pour réduire les couts des différents maillons technologiques et s'assurer de leur efficacité et de leur sécurité. L'infrastructure ECCSEL offrira des sites et bancs expérimentaux de pointe pour permettre aux chercheurs et ingénieurs européens de développer et tester de nouveaux outils, procédés et méthodes sur toute la chaîne de valeur : activités de captage, de transport et de stockage géologique de $\mathrm{CO}$, en ouvrant également la voie à des options de valorisation du CO 2. Elle permettra de coordonner et de structurer les efforts européens en matière de mise à disposition et d'investissements en infrastructures, rationalisant ainsi les efforts et les couts et renforçant le positionnement international de la recherche européenne.

ECCSEL-FR rassemble l'ensemble des équipements et infrastructures français en cours de coordination pour assurer la présence française dans l'infrastructure européenne ECCSEL:

- Stockage CO :

- BIOREP - BIO-Réacteur pour Environnements Profonds (BRGM), Orléans;

- LSBB - Laboratoire Souterrain à Bas-Bruit (CNRS), Rustrel:

- LS-Andra-Laboratoire souterrain de Meuse/Haute Marne (Andra), Bure;

- site de Catenoy dans I'Oise (INERIS), Catenoy;

- Transport CO :

- site de Mont La Ville dans l'Oise (INERIS), Mont-la-Ville;

- boucle de transport COOTRANS (TOTAL), Lacq-en projet

- Captage CO:

- pilote captage CO (EDF), Le Havre.

\section{IMPLICATIONSSOCIO-ÉCONOMIQUES}

L'infrastructure ECCSEL pourra nouer des partenariats avec différents acteurs privés et publics de la recherche et de l'innovation.

En France ils sont rassemblés au sein des structures suivantes : Club $\mathrm{CO}$, GIS GEODENERGIES, alliances ANCRE et ALLENVI, pôles de compétitivité AVENIA, TRIMATEC, RISQUES, OPTITEC, AXELERA, CAPENERGIES, TENERRDIS, EA éco-entreprises/GREEN.

\section{Coût de fonctionnement \\ $0,4 \mathrm{M€}$}

\section{Dimension internationale}

ECSSEL AS, ESFRI Roadmap

Coordinateur : NO

Pays partenaires : NO, UK, NL, FR, IT, PL, ES, EL, CH

Site internet : $w w w . e c c s e l . o r g$

\section{Coût RH}

13 ETPT 


\section{Infrastructure pour la recherche sur le solaire photovoltaïque/ SOlar PHotovoltaic Research Infrastructure}

Le solaire photovoltaïque est extrêmement diversifié, tant par les matériaux et process utilisés, que par les systèmes et applications permises. Une coordination des infrastructures aux niveaux national et européen est donc nécessaire pour permettre une meilleure efficacité des budgets nationaux de recherche. SOPHIRA a vocation à devenir le nœud français d'une infrastructure européenne.

Les équipements utilisés ou mis en place pour atteindre ces objectifs seront de plusieurs natures:

1. laboratoires avec salle blanche, équipements process et moyens de caractérisation avancés (TRL 2 à 4)

2. lignes pilotes, pour valider certains procédés et les méthodes de caractérisation en ligne, pour assurer l'extrapolation vers les capacités industrielles;

3. bancs tests et démonstrateurs multiples, avec retour d'expérience via une e-infrastructure, pour permettre le développement des logiciels de supervision, gestion, et maintenance.

Positionnement scientifique

1. domaines couverts par l'infrastructure

- procédés et caractérisation de matériaux, de cellules et modules solaires photovoltaïques:

- modélisation et développement de composants et de systèmes;

- modélisation de l'intégration à grande échelle dans les systèmes énergétiques:

2. missions et services proposés :

- validation de matériaux

- réalisation et validation d'architecture de cellules et de modules:

- développement de nouveaux procédés:

- analyse automatisée, à grande échelle, des performances des modules et des systèmes.

\section{IMPLICATIONSSOCIO-ÉCONOMIQUES}

Cette infrastructure comprendra des installations existantes et des extensions permettant des innovations sur toute la chaine de la valeur :

1. à l'amont, les fabricants de matériaux, d'équipements de production, et de cellules et modules

2. vers l'aval, tous les partenaires liés à l'intégration du solaire dans les réseaux électriques, les bâtiments et le transport, et à la fourniture des services.

\section{Coût de construction}

$10 \mathrm{M€}$

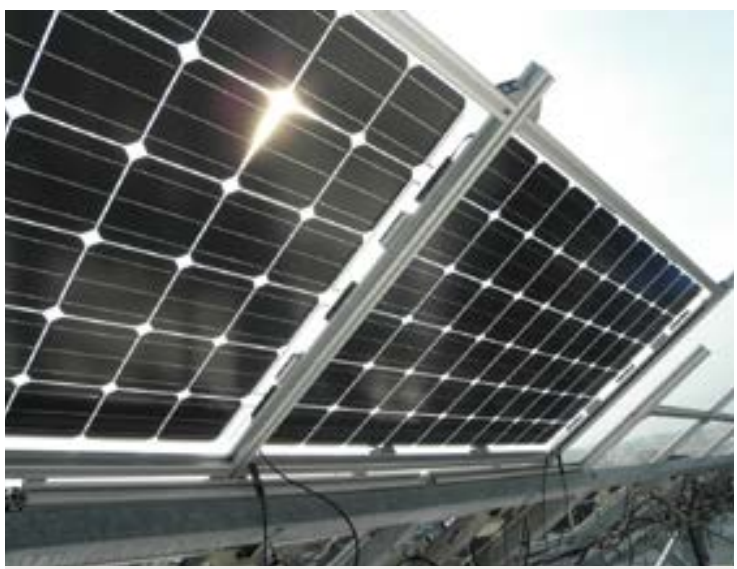

Type de l'infrastructure : Projet

\section{Localisation :}

Le Bourget-du-Lac

Responsable de l'infrastructure :

Philippe MALBRANCHE

Tutelles / Partenaires :

CEA

\section{Contact en France :}

philippe.malbranche@cea.fr

\section{www.sophia-ri.eu}




\section{Réseau de Moyens d'Essais en Hydrodynamique pour les Énergies Marines Renouvelables}

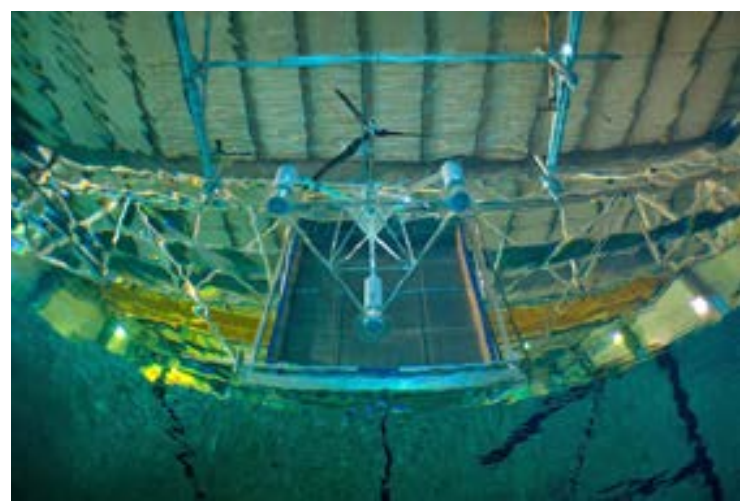

Type de l'infrastructure : Projet

Localisation de l'infrastructure:

Nantes, Brest

Localisation des autres sites :

Boulogne-sur-Mer, Le Croisic

Responsables de l'infrastructure:

Pierre FERRAND, Jean-Marc DANIEL

Création: Exploitation: Maintenance:

2015

2016

2016-2020

\section{Tutelles / Partenaires :}

École Centrale de Nantes, IFREMER

\section{Contact en France :}

Pierre.ferrant@ec-nantes.fr

Jean.Marc.Daniel@ifremer.fr

\section{wwz.ifremer.fr/rd \\ technologiques/Moyens/ \\ Moyens- $d$-essais/Bassin- $d$ - essais-du-centre-de-Bretagne}

wwz.ifremer.fr/institut_carnot

Iheea.ec-nantes.fr/doku.php/expe

www.semrev.fr
L'infrastructure est bâtie sur la mise en réseau des moyens d'essais en hydrodynamique de

- l'École Centrale de Nantes:

- Bassin de traction : Installé en 1977 et agrandi en 2000, il est le second bassin de ce type en France par sa taille;

- Bassin de Génie Océanique : Mis en service en 2000 et sans équivalent en France pour les essais sur houle, par la taille du bassin et les performances du générateur de houle;

- Bassin de Houle en eau peu profonde : Installé en 1982, reconditionné en 2014 par adjonction d'un faux fond pour les essais en profondeur limitée et mise en place d'un générateur de courant;

- SEMREV - Site d'essais à la mer (Le Croisic) : Seul site d'essais à la mer multi-technologies EMR opérationnel en France, développé depuis 2007, raccordé au réseau et instrumenté. Base à terre et équipe dédiée au Croisic (44):

- et de l'Ifremer

- Bassin de génie océanique (Brest) : Construit dans les années 70 et équipé d'un générateur de vagues. Essais en hydrodynamique et tests de matériel avant déploiement en mer. Unique en Europe par sa grande profondeur (10 m/20 m) et par un remplissage en eau de mer;

- Bassin à houle et courant (Boulogne-sur-Mer) : En 1990, I'Ifremer s'est doté d'une veine de circulation, installation unique en France et en Europe, dédiée à l'étude du comportement d'engins sous-marins. Equipée en 2010 d'un générateur de vagues pour la prise en compte des interactions houle-courant.

\section{IMPLICATIONSSOCIO-ÉCONOMIQUES}

L'émergence d'une filière industrielle EMR (sociétés d'ingénierie, de services, construction de machines, opérations en mer, maintenance) représente un secteur de développement prometteur au niveau régional mais aussi national et européen. L'infrastructure contribue au développement de cette filière au travers de nombreux projets de recherche partenariale avec les industriels du secteur.

\section{DONNÉES}

Présence dans des réseaux de données : Eurocean Marine Research Infrastructures Database (rid.eurocean.org)

\section{Coût de construction \\ - \\ $62 M €$ \\ Coût de fonctionnement : $1,2 \mathrm{M€}$}

\section{Dimension internationale}

En cours de développement, nœud français de l'IR proposée pour l'ESFRI Marinerg-i

Coordinateur: UCC-MaREI, IE

Pays partenaires : IE, PT, ES, UK, FR 
ÉNERGIE 

BIOLOGIE ET SANTÉ

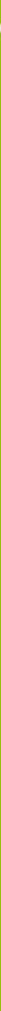




\section{Biologie et Santé}

Le domaine des sciences du vivant a connu des évolutions majeures et très rapides au cours des deux dernières décennies. Le changement d'échelle induit par l'essor des technologies, en particulier le séquençage génomique qui a ouvert l'ère de la biologie à haut débit ou l'imagerie haute résolution, a révolutionné l'approche de l'exploration du vivant.

Les technologies nécessaires, associées à des équipements coûteux, gérés par un personnel hautement qualifié, ont nécessité la mise en place d'infrastructures de recherche collectives et ouvertes à l'ensemble des communautés scientifiques.

Les infrastructures de recherche en sciences du vivant sont entrées pour la première fois dans la feuille de route nationale en 2008 , consacrant le succès d'une longue politique de structuration dans les années 90 . En 2010 et 2011, des appels d'offre (infrastructures nationales et démonstrateurs pré- industriels) ont été lancés dans le cadre du programme Biologie et Santé du Programme Investissement d'Avenir (PIA), pour développer les infrastructures technologiques afin de maintenir une recherche d'excellence dans le domaine des sciences du vivant. Les infrastructures de recherche présentées ici ont toutes été identifiées dans ce cadre. Certaines de ces infrastructures nationales sont les composantes françaises d'infrastructures européennes ESFRI, et participent ainsi à notre visibilité scientifique internationale et à la construction de l'espace européen de la recherche.

Certaines concernent des domaines d'intérêt conjoints avec AllEnvi, comme le Réseau de Centres de Ressources Biologiques pour les animaux (CRB Anim) qui fait partie de l'Infrastructure RARe (Ressource Agronomiques pour la Recherche), présentée dans la fiche environnement. Certaines des infrastructures listées sous le chapeau Sciences de la Matière et Ingéniérie (sources de rayonnement synchrotron, réseau RMN à très haut champs...) sont utilisées par les biologistes.

Les infrastructures de recherche en Biologie et Santé portent certaines spécificités comme celles d'être souvent distribuées sur plusieurs sites géographiques (mais avec une procédure d'accès centralisée) et d'être de taille modeste par rapport à la plupart des TGIR mono-sites de cette feuille de route. Elles n'en constituent pas moins des ensembles pouvant dépasser parfois 100 ETPT. Elles sont fortement soutenues par les établissements porteurs et partenaires. Leur pérennisation reste un défi qu'il faut relever au bénéfice des communautés scientifiques qui peuvent ainsi tirer parti de services compétitifs de haute technologie.

La France est membre de deux organisations internationales dans le domaine de la biologie:

I'EMBL qui, avec six sites de recherche, est l'un des grands centres d'excellence de la recherche fondamentale en biologie dans le monde.

L'EMBC qui fournit un cadre pour la coopération européenne dans le domaine de la biologie moléculaire et soutient des actions de formation, d'enseignement et de recherche et propose des bourses d'études.

Au niveau national, les infrastructures du périmètre «Biologie et Santé " sont organisées ici en 4 ensembles thématiques :

I. les IIR d'exploration fonctionnelle regroupent les technologies de pointe en matière d'étude des différentes composantes du vivant. L'un des enjeux de ces infrastructures est, dans une démarche de biologie des systèmes, de décrypter, à l'échelle d'une cellule, d'un tissu, d'un organe, ou d'un organisme vivant, le fonctionnement dynamique et coordonné d'un ensemble d'acteurs moléculaires. D'une part, l'intégration des différentes technologies expérimentales et computationnelles rend possibles des approches synergiques multi-résolutives dans l'espace et dans le temps. D'autre part, l'analyse multi-échelle de données hétérogènes, structurales mais aussi fonctionnelles, a pour but d'intégrer les aspects atomiques et cellulaires, voire tissulaires.

II. les IR d'organismes modèles et ressources proposent les modèles et échantillons biologiques nécessaires à l'étude du vivant. Les méthodes d'analyse de plus en plus résolutives et les nouvelles technologies d'ingénierie du génome (technologies CRISPR en particulier) nécessitent des approches d'analyse standardisées des phénotypes des organismes cibles et de disposer de ressources biologiques annotées dont la qualité et la tracabilité sont garanties. Certaines des infrastructures ont pour mission de fournir aux laboratoires les modèles nécessaires. D'autres permettent la mise à disposition de la communauté de cellules souches pluripotentes de qualité clinique et des collections de ressources biologiques humaines et microbiennes. 
La caractérisation de plus en plus fine de ressources biologiques permet désormais de proposer des approches inversées pour construire selon des procédés qualifiés, des entités ayant des activités biologiques recherchées, plasmides, enzymes, etc. Cette biotechnologie industrielle et/ou de synthèse qui est complémentaire de la biologie des systèmes, est un moteur important d'innovation dans la bio-économie, tant pour la santé et la nutrition que pour la chimie de synthèse.

III. les IR en investigation pré-clinique et clinique concernent la recherche transférable ou transférée à l'Homme.

Elles regroupent les structures qui permettent d'accélérer l'application des innovations médicales jusqu'aux personnes les plus susceptibles d'en bénéficier et les recherches effectuées sur un organisme modèle pré-clinique ou chez l'homme, volontaire sain ou porteur d'une maladie ou d'un pathogène. Sont associées à ces recherches toutes les infrastructures permettant d'accéder à l'élaboration de nouvelles approches thérapeutiques dans des conditions utilisables chez l'homme (GMP) ou garantissant sa sécurité (risque biologique).
IV. les IR en bioinformatique, les cohortes et les bases de données.

Les données produites en biologie et en médecine sont en augmentation exponentielle. L'Institut Français de Bioinformatique porte l'ambition de coordonner leur exploitation au plus près des laboratoires. Constances, une vaste cohorte en population générale, contribue à l'alimentation des données en santé pour la réalisation de projets dans des domaines variés (vieillissement et maladies chroniques, déterminants sociaux et professionnels de santé). Elle fournit aux agences et autorités de santé des données pour la surveillance des indicateurs de santé et la distribution des principaux déterminants de santé en population générale.

Le paysage des infrastructures en Biologie et en Santé étant très évolutif, I'amélioration des infrastructures existantes et le déploiement rapide des nouvelles technologies sont de première priorité. Il existe clairement des infrastructures émergentes dans plusieurs domaines technologiques (criblage et chimiothèques, neurosciences cognitives, cohortes, bases de données pour les recherches en santé...), qui ont vocation à être inscrits dans une prochaine révision de la feuille de route. Une partie a d'ailleurs reçu un soutien du PIA dans les programmes Cohortes ou Equipex et la création d'une infrastructure des données de santé découle de la loi de modernisation de notre système de santé. 


\section{LISTE DES INFRASTRUCTURES DE RECHERCHE DANS LE DOMAINE BIOLOGIE ET SANTÉ}

\begin{tabular}{|c|c|c|c|}
\hline CATÉGORIE & NOM & NOM COMPLET & ESFRI \\
\hline \multicolumn{4}{|c|}{ ORGANISATIONSINTERNATIONALES } \\
\hline OI & EMBL & $\begin{array}{l}\text { Laboratoire Européen de Biologie Moléculaire/ } \\
\text { European Molecular Biology Laboratory }\end{array}$ & \\
\hline OI & EMBC & $\begin{array}{l}\text { Conférence Européenne de Biologie Moléculaire/ } \\
\text { European Molecular Biology Conference }\end{array}$ & \\
\hline \multicolumn{4}{|c|}{ EXPLORATION FONCTIONNELLE } \\
\hline $\mathbb{R}$ & FBI & France-Biolmaging & EUBIO (2008) \\
\hline IR & FLI & France Life Imaging & EUBIO (2008) \\
\hline IR & France Génomique & $\begin{array}{l}\text { Infrastructure nationale de génomique } \\
\text { et bioinformatique associée }\end{array}$ & \\
\hline IR & FRISBI & $\begin{array}{l}\text { Infrastructure Française pour la Biologie } \\
\text { Structurale Integrée/French Infrastructure } \\
\text { for Integrated Structural Biology }\end{array}$ & INSTRUCT (2006) \\
\hline $\mathbb{I R}$ & MétaboHub & $\begin{array}{l}\text { Infrastructure française distribuée pour } \\
\text { la métabolomique dédiée à l'innovation, } \\
\text { à la formation et au transfert de technologie }\end{array}$ & \\
\hline IR & PROFI & $\begin{array}{l}\text { Infrastructure Française de Protéomique/ } \\
\text { Proteomics French Infrastructure }\end{array}$ & \\
\hline \multicolumn{4}{|c|}{ ORGANISMES MODËLES ETRESSOURCES } \\
\hline IR & BIOBANQUES & Infrastructure nationale BIOBANQUES & $\begin{array}{l}\text { BBMRI (2006) + } \\
\text { MIRRI }(2010)\end{array}$ \\
\hline IR & $\begin{array}{l}\text { CELPHEDIA/ } \\
\text { PHENOMIN }\end{array}$ & $\begin{array}{l}\text { Infrastructure Nationale en phénogénomique } \\
\text { de la souris }\end{array}$ & $\begin{array}{l}\text { INFRAFONTIER } \\
(2006)\end{array}$ \\
\hline IR & CELPHEDIA/TEFOR & $\begin{array}{l}\text { Trangénèse pour les Etudes Fonctionnelles } \\
\text { chez les ORganismes modèles }\end{array}$ & \\
\hline IR & CRB Anim & $\begin{array}{l}\text { Centres de Ressources Biologiques pour les } \\
\text { animaux domestiques }\end{array}$ & \\
\hline IR & EMBRC-France ${ }^{1}$ & $\begin{array}{l}\text { Centre National de Ressources Biologiques } \\
\text { Marines }\end{array}$ & EMBRC (2008) \\
\hline IR & EMPHASIS France ${ }^{2}$ & $\begin{array}{l}\text { European Multi-environment Plant pHenomics } \\
\text { And Simulation InfraStructure }\end{array}$ & \\
\hline IR & INGESTEM & $\begin{array}{l}\text { Infrastructure Nationale d'ingénierie des cellules } \\
\text { souches et des tissus }\end{array}$ & \\
\hline Projet & IBISBA-FR & $\begin{array}{l}\text { Industrial Biotechnology Innovation and Synthetic } \\
\text { Biology Accelerator }\end{array}$ & \\
\hline
\end{tabular}

1 Relève également du secteur « Sciences du système Terre et Environnement ».

2 Relève également du secteur "Sciences du système Terre et Environnement » dans lequel la fiche de l'infrastructure est présentée.

3 Relève également du secteur «Sciences du système Terre et Environnement ». 


\begin{tabular}{|c|c|c|c|}
\hline CATÉECORIE & \multicolumn{2}{|r|}{ NOM COMPLET } & ESFRI \\
\hline \multicolumn{4}{|c|}{ DU PRÉ-CLINIQUE AU CLINIQUE } \\
\hline IR & E-CellFrance & $\begin{array}{l}\text { Plateforme nationale pour la médecine } \\
\text { régénératrice basée sur les cellules souches } \\
\text { mesenchymateuses adultes }\end{array}$ & \\
\hline IR & F-CRIN & $\begin{array}{l}\text { Plateforme Nationale d'Infrastructures de } \\
\text { recherche Clinique/French Clinical Research } \\
\text { Infrastructure Network }\end{array}$ & ECRIN (2006) \\
\hline IR & Fr-Hadron & France HADRON & \\
\hline IR & HIDDEN & $\begin{array}{l}\text { Infrastructure de recherche dédiée aux maladies } \\
\text { hautement infectieuses/Highly Infectious } \\
\text { Diseases Dedicated Infrastructure ExtensioN }\end{array}$ & ERHINA (2008) \\
\hline $\mathbb{I R}$ & IDMIT & $\begin{array}{l}\text { Infrastructure nationale pour la modélisation des } \\
\text { maladies infectieuses humaines et les thérapies } \\
\text { innovantes }\end{array}$ & \\
\hline IR & NEURATRIS & $\begin{array}{l}\text { Infrastructure de Recherche Translationnelle } \\
\text { pour les Biothérapies en Neurosciences }\end{array}$ & EATRIS (2006) \\
\hline IR & PGT & $\begin{array}{l}\text { Consortium Préindustriel des vecteurs } \\
\text { de Thérapie Génique }\end{array}$ & \\
\hline
\end{tabular}

BIOINFORMATIQUE, COHORTES, BASES DE DONNÉES

\begin{tabular}{l|l|l} 
IR & CONSTANCES & $\begin{array}{l}\text { Cohorte des consultants des Centres d'examens } \\
\text { de santé }\end{array}$ \\
\hline IR & IFB & Institut Français de Bioinformatique
\end{tabular}




\section{Laboratoire Européen de Biologie Moléculaire/ European Molecular Biology Laboratory}

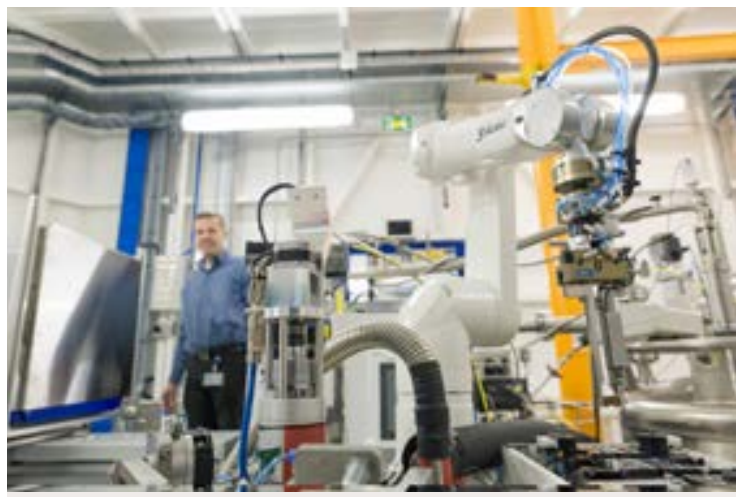

Type de l'infrastructure : OI

\section{Statut de l'infrastructure :}

Organisation Internationale

\section{Localisation :}

Heidelberg, DE

\section{Localisation des autres sites :}

Hambourg (DE), Grenoble (FR),

Monterotondo (IT), Hinxton (UK),

Barcelone (ES)

\section{Représentants en France :}

Jean-Michel HEARD, Elena HOFFERT

\section{Création :}

\section{4}

\section{Tutelles / Partenaires :}

22 états européens membres : AT, $C H, D E, D K$, FR, IL, IT, NL, SE, UK, FI, EL, NO, ES, BE, PT, IE, IS, HR, LU, CZ, MT

2 états non européens associés : AR et AU

\section{Contact en France :}

jean-michel.heard@recherche.gouv.fr elena.hoffert@recherche.gouv.fr
Avec ses 6 sites de recherche (Heidelberg, Hambourg, Grenoble, Monterotondo, Hinxton, Barcelone), I'EMBL est l'un des grands centres d'excellence de la recherche fondamentale en biologie dans le monde. Chacun des centres a un domaine de recherche spécifique : biologie et imagerie cellulaires, biologie structurale, développement de modèles murins, bioinformatique et biologie des systèmes.

Les grandes priorités stratégiques sont élaborées par un conseil scientifique et avalisées par l'assemblée générale. La priorité stratégique de I'EMBL pour la période 2017-2021 est la biologie digitale. Un Directeur Général assure la gestion scientifique de l'EMBL avec les coordinateurs de programme et les directeurs d'antennes.

Une centaine de jeunes scientifiques originaires des états membres, sélectionnés sur leur production scientifique antérieure, sont chef de groupe. EMBL leur offre des technologies de pointe, un environnement international très stimulant, et des possibilités de développer des collaborations scientifiques et de s'insérer dans des réseaux internationaux. Après avoir établi leur réputation, les chefs de groupe quittent l'EMBL après 4-5 ans et développent une activité de recherche indépendante.

L'EMBL développe un programme de doctorat en sciences de la vie (250 étudiants issus de plus de 40 pays) et un programme d'accueil de post-doctorants. L'EMBL organise de nombreux cours et conférences très réputés (EMBL courses and conferences, EMBL-EBI trainings).

\section{IMPLICATIONSSOCIO-ÉCONOMIQUES}

Valorisation et dissémination du savoir auprès de l'industrie font partie des préoccupations de l'EMBL. EMBLEM (EMBL Enterprise Management Technology Transfer), avec un statut de $\mathrm{GmbH}$, est le partenaire exclusif de l'EMBL pour le transfert technologique. Elle gère un portefeuille de plus de 250 brevets et copyrights (innovations technologiques, développements de programmes, bases de données).

\section{www.embl.fr}




\section{Conférence Européenne de Biologie Moléculaire/ European Molecular Biology Conference}

La Conférence Européenne de Biologie Moléculaire (EMBC) est une organisation inter-gouvernementale comprenant 27 pays membres (en pratique, la presque totalité des pays européens et quelques pays voisins).

L'EMBC fournit, à travers son Programme Général, un cadre pour la coopération européenne dans le domaine de la biologie moléculaire et des domaines de recherche voisins.

Ce Programme se décline principalement à travers la formation, l'enseignement et la recherche, par le biais de l'attribution de bourses d'études et la mise en place des programmes de cours, d'ateliers et de réunions de travail.

L'exécution du programme général de l'EMBC est confiée à l'EMBO (European Molecular Biology Organization). Les activités conjointes des deux organisations sont sous-tendues par un engagement commun pour une recherche de qualité au niveau européen, construite autour du développement de la coopération au sein de la communauté scientifique.

Les éléments qui composent le programme général de l'EMBC sont:

- le programme de bourses EMBO;

- les cours de l'EMBO et un programme d'ateliers:

- le programme Jeunes Investigateurs de l'EMBO;

- le programme de politique scientifique de l'EMBO

D'autres activités comprennent:

- les Symposia EMBO | EMBL;

- un programme de développement de carrière:

- les conférences EMBO

- un programme EMBO de promotion des femmes dans la science; - un programme de bourses de développement stratégique (SDIG);

- les publications scientifiques EMBO

\section{IMPLICATIONSSOCIO-ÉCONOMIQUES}

L'EMBO est une organisation à but non lucratif mais, par le biais des différentes formes de formation qu'elle dispense, elle contribue pleinement au développement d'une économie basée sur la connaissance en Europe et dans le reste du monde.

\section{Coût de fonctionnement}

$2015: 19 \mathrm{M} €$

dont 2,9M€ de contribution

française

\section{Dimension internationale}

Coordinateur : Gerrit van Meer, NL

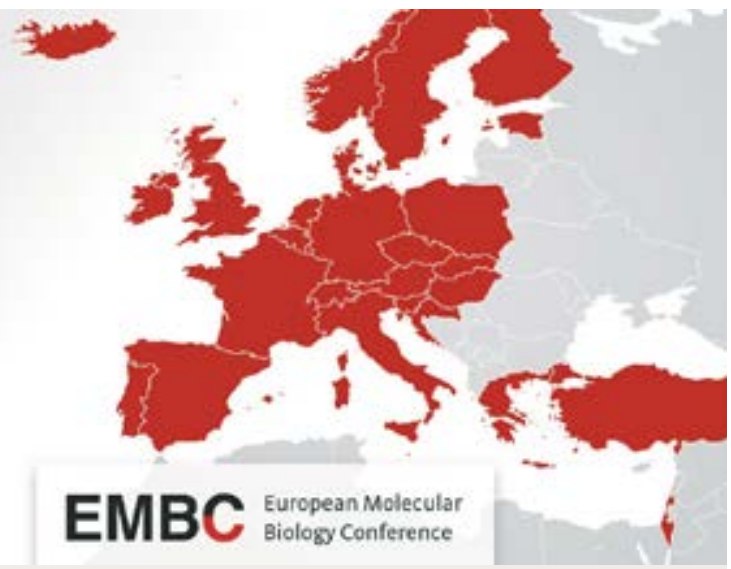

Type de l'infrastructure : OI

Statut de l'infrastructure :

Organisation Internationale

\section{Localisation :}

Heidelberg, DE

\section{Représentants en France :}

Jean-Michel HEARD, Elena HOFFERT

\section{Création :}

1969

\section{Tutelles / Partenaires :}

27 états membres: AT, BE, HR, CZ,DK, EE, FI, FR, DE, EL, HU, IS, IE, IL, IT, LU, NL, NO, PL, PT, SK, SI, ES, SE, CH, TR, UK

\section{Contact en France :}

jean-michel.heard@recherche.gouv.fr elena.hoffert@recherche.gouv.fr

\section{embc.embo.org}




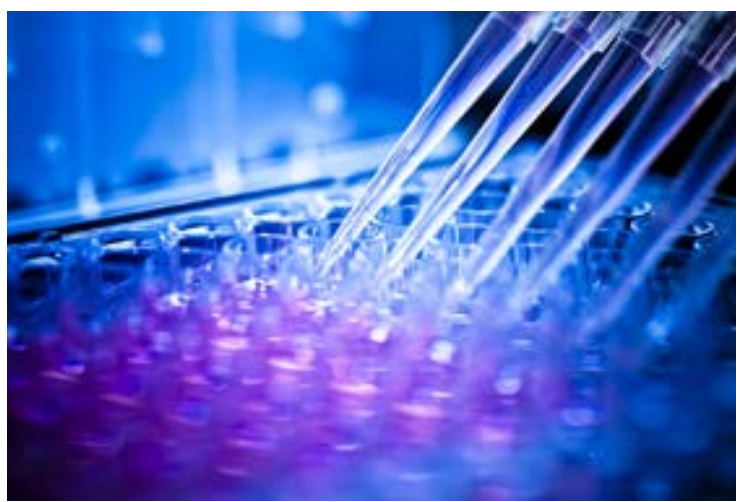

Type de l'infrastructure : IR

\section{Localisation de l'infrastructure: \\ Paris}

Localisation des autres sites :

94 centres de ressources biologiques

\section{Responsable de I'infrastructure : Georges DAGHER}

Création: Exploitation:

2011 2014

\section{Tutelles / Partenaires :}

Inserm, INRA, Institut Pasteur, CEA, CNRS, CNCR

\section{Contact en France:}

biobanques@inserm.fr

\section{www.biobanques.eu}

Infrastructure répartie sur l'ensemble du territoire, BIOBANQUES regroupe 94 centres de ressources biologiques et couvre tous les champs de la recherche biomédicale avec plus de 700 projets de recherche utilisant les collections biologiques. En interface avec les infrastructures européennes BBMRI-ERIC, MIRRI et les projets Investissement d'avenir, elle participe à la cohésion de la recherche en biologie au niveau national et international.

BIOBANQUES traduit les priorités stratégiques des grands acteurs en sciences de la vie et de la santé en France définies par les Alliances AVIESAN et ARIIS, ainsi que la stratégie nationale des infrastructures de recherche:

- elle favorise la recherche translationnelle, le développement des biomarqueurs et la médecine personnalisée;

- elle participe au renforcement des plateformes pluridisciplinaires d'excellence, qui permettent de mutualiser les moyens et les compétences, d'accroitre les investissements dans les secteurs prioritaires pour la santé publique et pour le développement économique;

- elle favorise les partenariats public-privé pour renforcer les développements technologiques innovants et la recherche translationnelle.

A cet effet, l'infrastructure favorise:

- le développement de consortia nationaux et internationaux;

- l'échange de ressources biologiques et de données associées;

- I'accès aux ressources via une procédure unique;

- le contrôle qualité des échantillons;

- l'interopérabilité des bases de données cliniques et biologiques.

\section{IMPLICATIONSSOCIO-ÉCONOMIQUES}

Biobanques a contribué au développement de plateformes de technologies innovantes:

- une plateforme de conservation de I'ADN à l'ambiante sous gaz neutre; - la traçabilité de l'échantillon par RFID.

Elle envisage pour 2016-2017 le développement de la conservation de I'ARN sous gaz neutre, la robotisation du parcours de l'échantillon, le développement d'outils informatiques pour la médecine personnalisée.

\author{
Dimension internationale \\ Biobanking and Biomolecular research Infrastructure (BBMRI-ERIC), \\ ESFRI Landmark \\ Microbial Resources Research Infrastructure (MIRRI), ESFRI ESFRI Roadmap \\ Coordinateurs: \\ BBMRI-ERIC : Jan-Eric Litton \\ MIRRI : Erko Stackebrandt \\ Pays partenaires : \\ BBMRI-ERIC : 19 pays de I'UE \\ MIRRI : 19 pays de I'UE \\ Site internet : \\ www.bbmri-eric.eu \\ www.mirri.org
}




\title{
CE LP

\section{Infrastructure Nationale en phénogénomique de la souris}

La complexité des relations entre génome et environnement avec pour conséquence l'adaptation du phénotype d'un individu dans son milieu a conduit à développer PHENOMIN, une infrastructure dédiée à la communauté scientifique afin:

1. d'augmenter la connaissance du génome des mammifères en étudiant la fonction des gènes dans des processus intégrés (développement, physiologie, comportement, vieillissement, réponse inflammatoire...) ainsi que la fonction de gènes non annotés ou de régions non codantes (96\% du génome);

2. d'identifier et comprendre les variations du génome à l'origine de maladies, les mutations pathogènes à l'origine de maladies mono et multi géniques et dues aussi bien à des mutations ponctuelles qu'à des allèles rares ou du nombre de copies d'un gène (trisomie, syndromes de micro délétion, CNVs);

3. découvrir de nouvelles opportunités pour l'innovation et le développement de thérapies ou de molécules pour répondre à la demande de la société (immunothérapies anti-tumorales...).

Ces objectifs majeurs sont poursuivis avec la mise en place d'un haut niveau de mutualisation et d'intégration au travers d'un portail d'accès unique; des actions de formation et de communication conjointes pour renforcer la visibilité nationale et internationale; et une offre de services à haute valeur ajoutée pour la création, l'élevage, la préservation, l'analyse fonctionnelle multivariée et le test de nouvelles thérapies, ouverteàl'ensemble de la communauté académique et privée; le tout dans le respect des règles d'éthique et du bien-être animal.

\section{IMPLICATIONSSOCIO-ÉCONOMIQUES}

Environ $10 \%$ de l'activité de PHENOMIN est réalisée pour des partenaires privés. De plus, PHENOMIN travaille avec une dizaine de partenaires industriels en recherche et développement dans les domaines de nouvelles thérapies contre des maladies uro-génitales, tumorales et rares, d'outils innovants pour l'hébergement, de prototypes de microscopie ou d'appareils et de stratégies d'imagerie in vivo.

\section{DONNÉES}

Présence dans des réseaux de données : INFRAFRONTIER

\section{Coût de construction}

$65 \mathrm{M} €$

Coût de fonctionnement : $14 \mathrm{M} €$

\section{Coût RH \\ 109,6 ETPT}

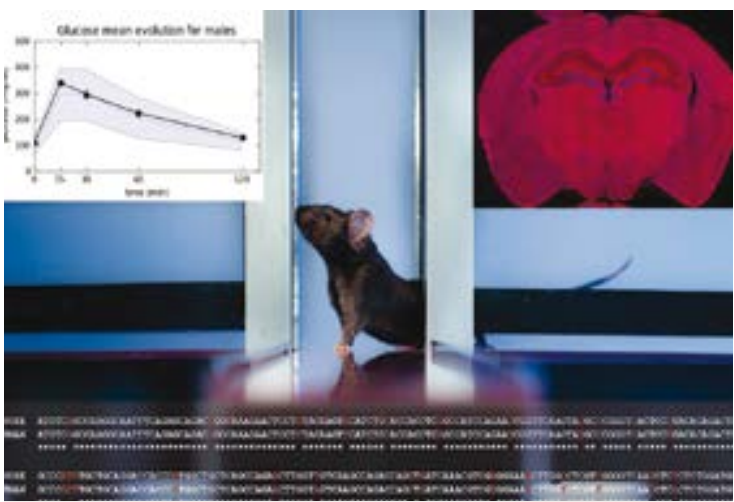

Type de I'infrastructure : IR

\section{Localisation :}

Illkirch

Localisation des autres sites :

Marseille, Luminy, Orléans, Villejuif

Responsable de l'infrastructure :

Yann HERAULT

\section{Création :}

Exploitation: Maintenance :

2011

2011

2018

\section{Tutelles / Partenaires:}

CNRS, INSERM, Univ. Strasbourg, AMU, CERBM-GIE

\section{Contact en France:}

herault@igbmc.fr

contact@phenomin.fr

\section{www.phenomin.fr}

\author{
Dimension internationale \\ Membre fondateur d'INFRAFRONTIER, ESFRI Landmark \\ Membre fondateur de l'International Mouse Phenotyping consortium (IMPC) \\ Coordinateur: \\ INFRAFRONTIER : Martin Hrabé de Angeli \\ IMPC : Mark Moore \\ Pays partenaires: \\ INFRAFRONTIER : DE, FR, CZ, FI, EL \\ IMPC : UK, FR, DE, IT, ES, CZ, JP, KR, CN, AU, USA, CA, EMBL \\ Site internet : \\ www.infrafrontier.eu/www.mousephenotype.org
}




\section{CELPHEDIA/TEFOR \\ Transgénèse pour les Etudes Fonctionnelles chez les ORganismes modèles}

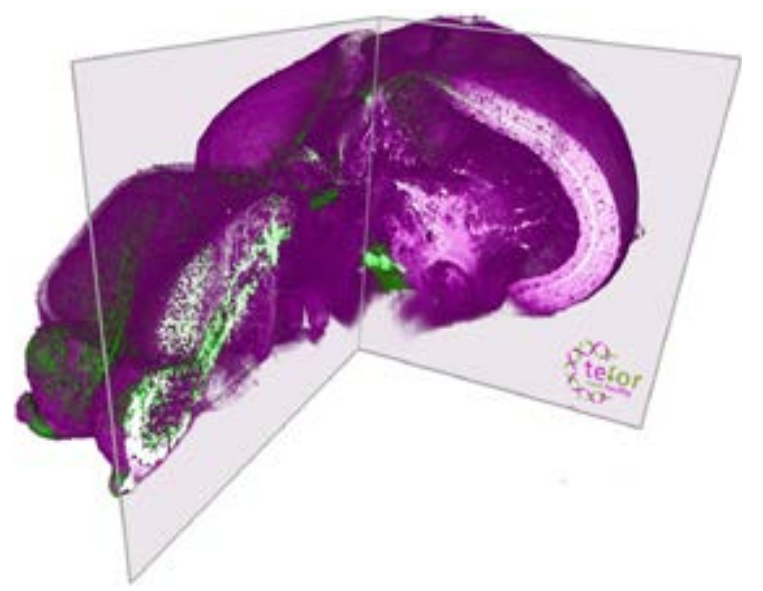

Type de l'infrastructure : IR

Statut de l'infrastructure :

Unité mixte de recherche

Localisation de l'infrastructure:

Gif-sur-Yvette

Localisation des autres sites:

Paris, Jouy-en-Josas, Versailles, Versailles,

Rennes, Nantes, Clermont-Ferrand

\section{Responsable de l'infrastructure :}

Jean-Stéphane JOLY

\section{Création: Exploitation: \\ 2011 2013}

\section{Maintenance :}

Chaque année

\section{Tutelles / Partenaires :}

CNRS, INRA, INSERM, MNHN, Univ. Auvergne, UPB, Univ. Nantes

\section{Contact en France :}

djian@tefor.net

\section{www.tefor.net}

TEFOR est une infrastructure distribuée : 11 plateformes et laboratoires experts se sont associés pour fournir des services innovants en édition du génome, transgénèse, mutagénèse, et phénotypage par imagerie. Bien que TEFOR soit à ce jour centrée sur les modèles poisson-zèbre et drosophile, elle reste ouverte aux autres organismes modèles, en particulier via son réseau d'animation scientifique EFOR (www.efor.fr), mais aussi par ses nombreuses collaborations

Pour assurer la qualité continue de ses services, TEFOR s'est engagée à mener une recherche technologique de pointe, notamment pour améliorer I'utilisation du système CRISPR/Cas9 pour l'édition du génome. Elle conduit également des programmes de phénotypage systématique à grande échelle sur des lignées fluorescentes. Ces programmes sont accompagnés du développement de collections d'images 3D et d'outils de navigation optimaux dans des bases de données.

Pour servir la communauté française, européenne et internationale, TEFOR développe un point d'accès unique pour un ensemble de services intégrés: - un service distribué pour la création de lignées modifiées via le système CRISP-R/Cas9;

- des services de transgénèse innovants;

- des services de phénotypage par imagerie, à basse ou haute résolution, 2D/3D/4D (3D+T);

- I'archivage des images dans des bases de données dédiées, avec une navigation aisée via une interface web conviviale.

\section{IMPLICATIONSSOCIO-ÉCONOMIQUES}

TEFOR a collaboré avec la société Leica-Microsystems@ $\odot$ pour le développement d'un nouvel outil appelé "VibMic » (microscope confocal couplé à un vibratome). D'importants efforts ont été menés pour l'optimisation des méthodologies de transparisation de tissus. Ces innovations permettent une imagerie profonde à haute résolution. TEFOR compte parmi ses utilisateurs plusieurs laboratoires privés.

\section{DONNÉES}

Stockage : Stockage des données d'imagerie sur des serveurs spécifiques de TEFOR

Accessibilité : Certaines données peuvent être en libre accès (ressources gratuites) et d'autres restreintes (pour certains usagers dans le cadre de services). 


\section{Cohorte des consultants des Centres d'examens de santé}

Constances est une cohorte épidémiologique prospective de 200000 sujets représentative de la population âgée de 18 à 69 ans. L'inclusion a lieu dans des Centre d'examens de santé dans 20 départements métropolitains, comprenant un examen de santé, la constitution d'une biobanque et des questionnaires (santé, mode de vie, facteurs socio-professionnels). Le suivi est actif (autoquestionnaire annuel, examen tous les 5 ans), et passif par appariement annuel avec les bases de données de la Cnav, du SNIIRAM et des causes de décès. Les principales données recueillies concernent la santé, le recours aux soins, des paramètres biologiques et physiologiques, une biobanque, les caractéristiques sociodémographiques, les facteurs professionnels. La constitution de la cohorte complète est prévue sur 6 ans; en décembre 2015, 87000 participants sont inclus. Une cohorte témoin de 400000 personnes est suivie dans les bases de données nationales pour permettre le redressement des estimations.

C'est une infrastructure «immatérielle» dont la mission consiste essentiellement à recueillir de façon prospective des données individuelles des participants à de multiples sources. Par l'intermédiaire d'appels à projets, la base de données de la cohorte est ouverte à la communauté de recherche française et internationale; plus de 50 projets ont été validés par le Conseil scientifique international de Constances.

Constances est la plus importante cohorte française, et participe à plusieurs consortiums européens.

\section{IMPLICATIONSSOCIO-ÉCONOMIQUES}

La construction de la cohorte fait l'objet d'un partenariat avec la CNAMTS et la Cnav qui fournissent des données. Constances est utilisée par divers organismes de santé publique (agences sanitaires, ministère, mutuelles, assurance maladie). Des partenariats ont été établis avec plusieurs entreprises industrielles du domaine du médicament et des biotechnologies.

\section{DONNÉES}

Estimation du flux de données : 40000 nouveaux participants inclus chaque année.

Stockage : 15 téraoctets au total pour la cohorte à 5 ans de suivi

Accessibilité : Transmission de fichiers cryptés par internet (autorisation CNIL indispensable)

Présence dans des réseaux de données : Infrastructures françaises : BIOBANQUES E $M E T A B O H U B$

Participation à des consortiums européens de cohortes en population avec partage de données : BBMRI-LPC, IDEAR, LIFEPATH.

\section{Coût de construction}

24 Me

Coût de fonctionnement : $6 \mathrm{M€}$

\section{Coût RH}

-

\section{$1,8 \mathrm{ME}$}

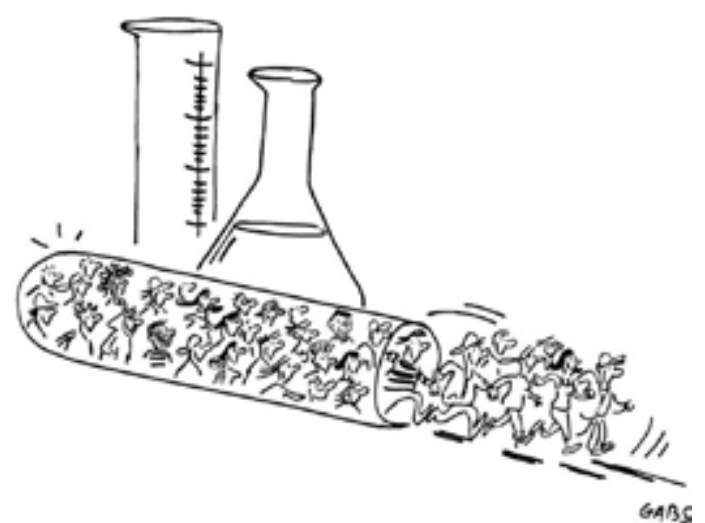

Type de l'infrastructure : IR

\section{Responsable de l'infrastructure:}

Marie ZINS

Création :

\section{Exploitation :}

2012 2014

\section{Maintenance :}

Permanent

\section{Tutelles / Partenaires :}

CNAMTS, CNAV, INSERM, UVSQ

Contact en France:

marie.zins@inserm.fr

www.constances.fr 


\section{CRB-ANIM}

\section{Centres de Ressources Biologiques pour les animaux domestiques}

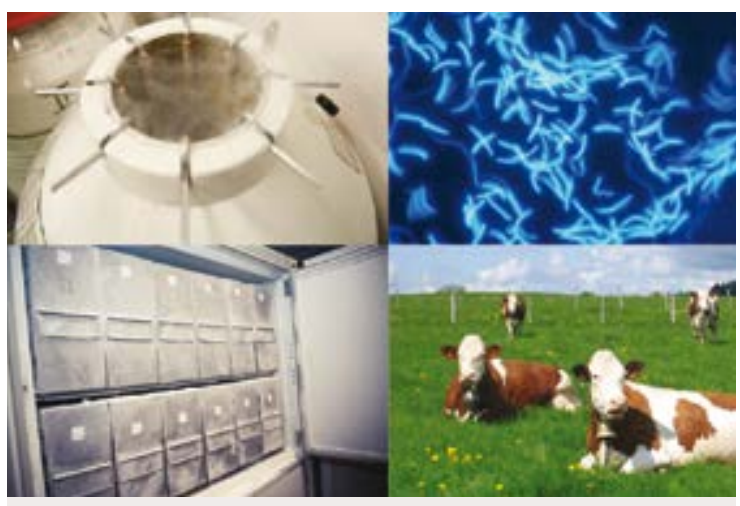

Type de l'infrastructure : IR

\section{Localisation de l'infrastructure:}

Jouy-en-Josas

Localisation des autres sites :

Maisons-Alfort, Rennes, Lyon

Responsable de l'infrastructure/

Représentant en France :

Michèle TIXIER-BOICHARD

Création :

Exploitation :

2012

2017

\section{Tutelles / Partenaires :}

INRA, CNRS, FRB, VetAgro Sup.

\section{Contact en France :}

contact@crb-anim.fr

\section{www.crb-anim.fr}

Les missions de CRB-Anim sont de collecter, caractériser, conserver et distribuer des ressources biologiques non infectieuses, pour 22 espèces animales domestiques et les espèces apparentées sauvages. Les espèces animales domestiques sont des espèces dont l'évolution est fortement influencée par l'action de l'homme : elles font l'objet de programmes de sélection et/ou de gestion de leur variabilité génétique. L'infrastructure soutient les recherches concernant l'évaluation de la diversité génétique et de la réponse à la sélection, ainsi que l'identification de gènes contrôlant des phénotypes d'intérêt ou des anomalies génétiques (modèles biomédicaux). Elle permet aussi la reconstitution de populations.

Des développements technologiques sont réalisés pour faciliter la collecte de matériel biologique, améliorer la qualité reproductive du matériel collecté et sa facilité d'utilisation. CRB-Anim propose des protocoles de prélèvement et de conservation des échantillons et offre un service d'extraction d'ADN de haute qualité pour une gamme d'espèces et de tissus, fluides et excrétat (sang, peau, muscle, nageoire, fèces, sperme). II apportera un soutien organisationnel à ses membres notamment dans l'application du protocole de Nagoya.

Le portail web de l'infrastructure, encore en développement, offrira un service de demande d'entrée ou de sortie d'échantillons avec des descripteurs minimaux et des métadonnées pour faciliter les requêtes.

\section{IMPLICATIONS SOCIO-ÉCONOMIQUES}

\section{Partenariat avec deux entreprises de biotechnologies LABOGENA et ANTAGENE.}

Développement de méthodes de congélation de semence ou d'embryons transférables à des sociétés de biotechnologies de la reproduction.

\section{DONNÉES}

Estimation du flux de données : Volume $<1$ To.

Stockage : Les données sont stockées par chaque membre de CRB Anim. Accessibilité : Accès libre aux données.

Présence dans des réseaux de données : L'identification des jeux de données est prévue dans le cadre de GBIF. 


\section{Plateforme nationale pour la médecine régénératrice basée sur les cellules souches mésenchymateuses adultes}

ECELLFRANCE a pour mission le développement en France des thérapies cellulaires basées sur l'utilisation des cellules souches mésenchymateuses (CSM) adultes. Ces thérapies visent à régénérer les tissus endommagés dans de nombreuses pathologies liées à l'âge et de maladies chroniques inflammatoires actuellement sans traitement curatif.

L'infrastructure est structurée autour des acteurs clefs de la médecine régénérative et a adopté un positionnement fort sur la recherche translationnelle vers la clinique. ECELLFRANCE est notamment équipée de centres de production de CSM et de plateformes de suivi clinique d'immunomonitoring de patients traités par CSM. L'organisation regroupe ainsi des équipes de recherche spécialisées dans la thérapie cellulaire, des centres hospitaliers pour la recherche clinique et translationnelle, et des centres de production de médicaments de thérapie innovante (MTI).

Plus spécifiquement, ECELLFRANCE a pour mission d'optimiser et d'harmoniser les différentes étapes nécessaires au développement des «cellules souches médicament » et de la médecine régénératrice en France, et de proposer aux équipes académiques ou industrielles d'accélérer leur programme de R\&D en les accompagnant dans les différentes phases de leur projet de thérapie cellulaire : validation du projet, réalisation d'études précliniques, montage de dossiers réglementaires, production de CSM à usage clinique, mise en place d'essais cliniques de phase l et II avec suivi des patients.

\section{IMPLICATIONSSOCIO-ÉCONOMIQUES}

20 programmes d'essais cliniques nationaux et européens en thérapie cellulaire, pour moitié porteurs externes académiques et industriels. En dehors de l'enjeu de santé publique et des impacts cliniques et sociétaux associées à son activité, 4 partenariats industriels avec des applications diverses (cosmétique, logiciel d'analyse de données, test d'analyse biologique). Création d'une CMO (T3P) prévue début 2016.

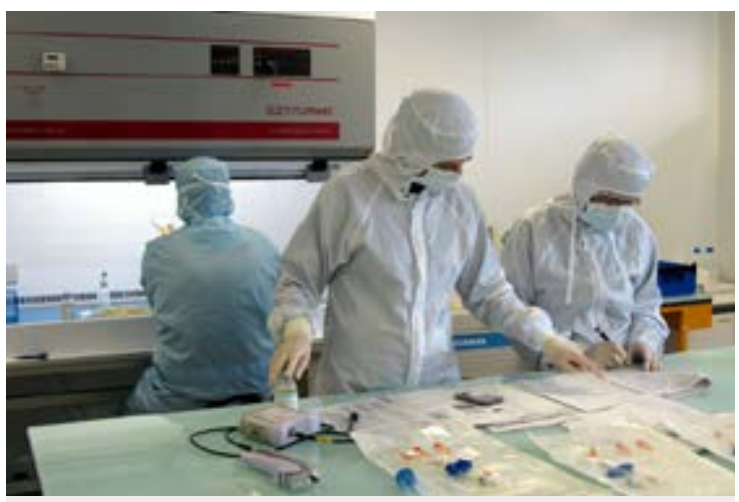

Type de l'infrastructure : IR

\section{Localisation :}

Montpellier

\section{Localisation des autres sites :}

Toulouse, Clamart, Grenoble, Besançon, Créteil, Rennes

Responsable de l'infrastructure :

Christian JORGENSEN

Création:

Exploitation :

2012 2012

\section{Tutelles / Partenaires :}

Univ. Montpellier, CTSA, CNRS, EFS, INSERM, Univ. Toulouse, GIN Grenoble, CHU de Grenoble

\section{Contact en France :}

contact@ecellfrance.com

www.ecellfrance.com 


\section{EMBRC-FRANCE}

\section{Centre National de ressources biologiques marines}

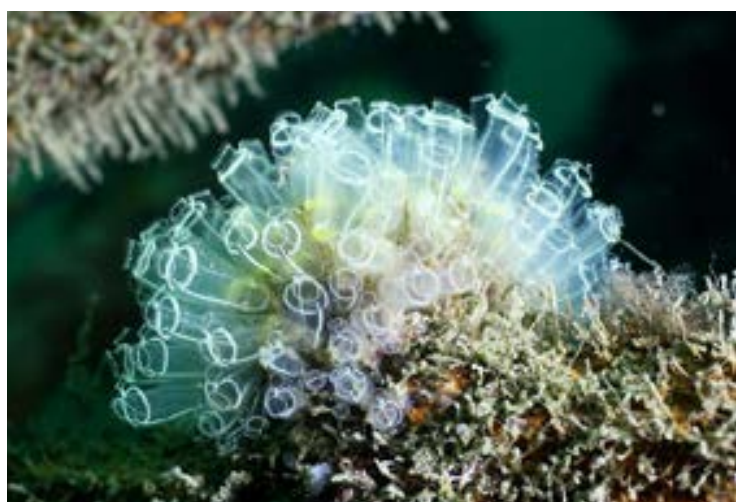

Type de l'infrastructure : IR

\section{Localisation de l'infrastructure :}

Roscoff

Localisation des autres sites :

Banyuls, Villefranche-sur-Mer

\section{Responsable de l'infrastructure :} Bernard KLOAREG

Création: Exploitation:

2011 2015

Tutelles / Partenaires : UPMC, CNRS

\section{Contact en France :}

kloareg@sb-roscoff.fr

www.embrc-france.fr
Les Stations marines de Roscoff, Banyuls et Villefranche-sur-Mer fournissent toutes les trois, mais avec diverses spécificités, des moyens à la mer (bateaux, marins, plongeurs...), des aquariums et laboratoires alimentés en eau de mer courante, des plates-formes analytiques et des structures d'hébergement. Leur principale caractéristique est de pouvoir fournir à la communauté scientifique des modèles microbiens, animaux ou végétaux qui représentent des lignées évolutives majeures et qui ne sont pas présentes dans les écosystèmes terrestres

Les recherches sur la biologie des organismes marins vivent une véritable révolution, conceptuelle et technologique. Les approches de génomique sensu lato sont en effet désormais disponibles sur de nombreux organismes ainsi que sur les écosystèmes marins. Pour plusieurs lignées au moins, les approches d'analyse fonctionnelle sont désormais au niveau de celles pratiquées sur les modèles biologiques terrestres.

EMBRC-France est construit autour des services suivants:

- accès aux modèles biologiques marins, couvrant toutes les lignées de l'arbre du vivant;

- accès à des dispositifs d'expérimentation ex situ, pour l'élevage ou la culture;

- accès aux moyens logistiques pour le génotypage et le phénotypage de ces modèles (-omiques, imagerie, performances en culture...);

- accès à des ressources génétiques de certains organismes modèles procaryotes ou eucaryotes;

- accès à des ressources numériques sur les organismes et les écosystèmes marins.

\section{IMPLICATIONSSOCIO-ÉCONOMIQUES}

L'infrastructure arrive en appui de l'innovation sur les bio-ressources marines elles-mêmes (microbiennes, végétales ou animales), les produits bio-sourcés (biomolécules, bio-raffineries), et les systèmes de production (des usines cellulaires à l'aquaculture). Les secteurs d'application sont très vastes, du développement de composés bio-actifs jusqu'à l'amélioration des espèces aquacoles.

\section{DONNÉES}

Présence dans des réseaux de données : IFB, France Génomique, European Marine Biological Infrastructure Cluster (EMBRIC).

\section{Dimension internationale \\ "European Marine Biological Resources Center » (EMBRC), ESFRI Roadmap Coordinateur : Ilaria Nardello, FR \\ Pays partenaires : BE, EL, IL, IT, PT, NO, UK \\ Site internet : www.embrc.eu}




\section{France-Biolmaging}

La mission principale du FBI est de permettre l'accès aux dernières innovations en Imagerie des sciences du vivant. Cette infrastructure multidisciplinaire:

- explore de nouvelles voies en bio-imagerie et encourage leurs applications en sciences biologiques. Elle est un outil pour la recherche dans de nombreux domaines, de la recherche sur la biologie des plantes ou les cellules souches aux études précliniques et translationnelles sur le cancer ou les maladies neurodégénératives;

- vise à favoriser le transfert technologique des innovations réalisées par ses équipes expertes de $R \& D$, vers ses Plateformes. Elle a pour vocation d'en accélérer l'accès, tout en assurant le contrôle de la qualité et la pérennité, des systèmes d'acquisition et des données produites:

- est un portail d'entrée pour des projets privés/publics dans le domaine de la microscopie avancée, en particulier par l'utilisation des installations d'imagerie pour le criblage et le développement de médicaments et de principes actifs en cosmétique

est un centre de ressource pour la formation et l'éducation. FBI soutient les activités de dissémination en imagerie biologique, organise et participe à des programmes nationaux et internationaux de formation (FBI-AT; EMBO cours et ateliers, réunions Elmi, MiFoBio scolaires CNRS...); est avec ses réseaux et structures frères, impliqué dans des programmes H2O2O (InfraDEVSUP II Euro-Biolmaging, RIA Global Biolmaging, COST projects...) (formation, gestion des données «images »...).

\section{IMPLICATIONS SOCIO-ÉCONOMIQUES}

Plus de 30 partenariats (Imagine Optics, PHASICS, Strand Avadis...). Utilisation des plateformes d'imagerie pour le criblage et le développement en pharmacologie et en cosmétique (L'Oréal, Clarins, Sanofi, Servier, Roche...); 18 licences et brevets en 2014-2015. Mentorat et soutien des jeunes entreprises (BioAxial, CryoCapcell, QuantaCell...).

\section{DONNÉES}

Estimation du flux de données : Quelques Po/an.

Stockage : 50-200 To/nœud de l'infrastructure. FBI développe des Centres de Données, des Bases de Données Images communes sécurisées (CiD-iManage), liées à des plateformes logicielles.

Accessibilité : Via des bases de données d'images en local. Une norme des infrastructures européennes est en projet.

Présence dans des réseaux de données : Via EuBiolmaging et Elixir, $\mathrm{FB}$ est engagé dans la gestion des données « image ». EuBI soutient le consortium " BioMedBridges " qui tend à consolider la gestion et l'interopérabilité des ressources électroniques en sciences biomédicales.

\section{Coût de construction \\ $46 \mathrm{M} €$ \\ Coût de fonctionnement : $4 \mathrm{M€}$}

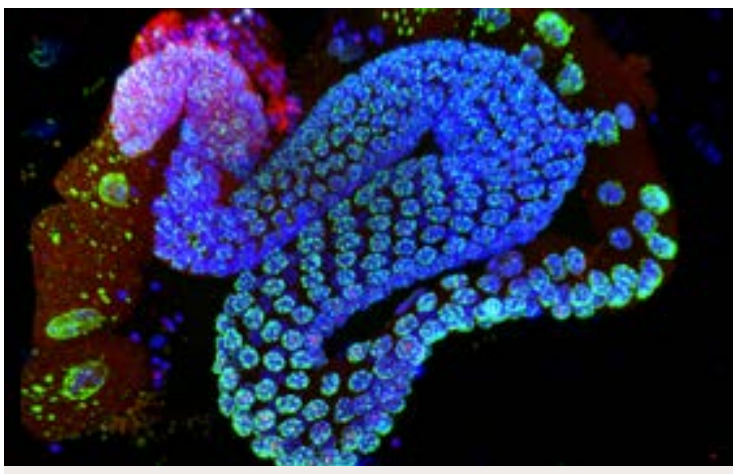

Type de l'infrastructure : IR

Statut de l'infrastructure :

Unité mixte de service

\section{Localisation :}

Paris

\section{Localisation des autres sites :}

Marseille, Montpellier, Bordeaux, Gif sur Yvette, Rennes

\section{Responsable de l'infrastructure : Jean SALAMERO}

\section{Création: Exploitation: \\ 2011 2013}

\section{Maintenance:}

Phase d'investissement : 2012-2017

\section{Tutelles / Partenaires :}

CNRS, ENS, Ecole Polytechnique, Inria, Inserm, Institut Curie, Institut Pasteur, AMU, Univ. Bordeaux, Univ. Montpellier, Univ. Paris VII, Univ. Paris Descartes, UPMC

\section{Contact en France:}

contact@france-bioimaging.org

\section{france-bioimaging.org}

\section{Dimension internationale \\ Euro-Biolmaging, ESFRI Roadmap. \\ Pays partenaires : BEBG, CZ,FI, FR, IT,IL, NL, NO, PO, PT,SK, SE,UK, EMBL \\ Site internet: $w$ ww.eurobioimaging.eu}




\section{Plateforme Nationale d'Infrastructures de recherche clinique/ French Clinical Research Infrastructure Network}

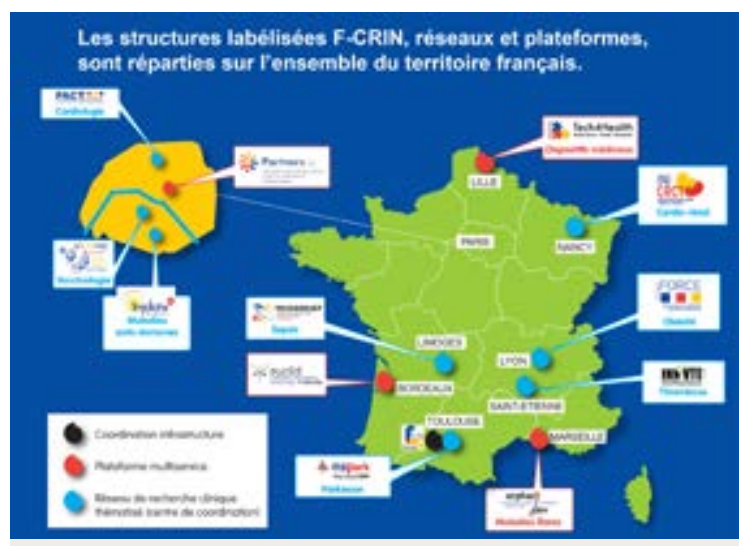

Type de l'infrastructure : IR

\section{Statut de l'infrastructure :}

Unité mixte de service

Localisation de l'infrastructure:

Toulouse

Localisation des autres sites :

54 centres d'investigation clinique nationaux $(\mathrm{CIC})$

Responsable de l'infrastructure/

Représentant en France :

Olivier RASCOL, Vincent DIEBOLT

\section{Création: Exploitation :}

2012

$$
2012
$$

Tutelles / Partenaires :

Inserm, CHU de Toulouse, Univ. Toulouse

\section{Contact en France :}

contact@fcrin.org

\section{www.fcrin.org}

Mise en place en 2012, «F-CRIN » est lauréate de l'édition 2010 de l'appel à projets : «Infrastructures Nationales en biologie et en santé » lancé dans le cadre des «Investissements d'avenir ».

Composante française de I'ERIC «ECRIN/European clinical research infrastructure network », réseau européen de recherche clinique, elle en constitue à ce titre le point d'entrée national.

La raison d'être de F-CRIN est de renforcer les performances de la recherche clinique française et donc son attractivité à l'international, son implication dans les appels à projets européens et les essais translationnels et cliniques multinationaux.

F-CRIN est une «infrastructure support» en appui des investigateurs et des promoteurs académiques, hospitaliers et industriels.

Infrastructure distribuée, F-CRIN associe 13 composantes:

- 4 Plateformes nationales ( 2 généralistes; 2 spécialisées: dispositifs médicaux et maladies rares) ayant la masse critique pour offrir un menu complet de prestations, de l'aide à la conception et au montage d'essais cliniques jusqu'à l'analyse des données recueillies;

- 8 réseaux d'excellence en recherche clinique affichant un programme scientifique original et attractif au plan international dans des thématiques à fort potentiel de développement, et disposant d'un savoir-faire collectif avec une forte capacité d'investigation;

- une coordination localisée à Toulouse qui assure la représentation nationale de I'Infrastructure «F-CRIN» et assure des services d'intérêt commun.

\section{IMPLICATIONSSOCIO-ÉCONOMIQUES}

L'Infrastructure nationale en recherche clinique « F-CRIN » a trois domaines d'actions ciblés :

- les études cliniques multicentriques complexes;

la recherche clinique translationnelle et la preuve de concept;

les projets de Recherche Clinique Européens.

Elle a également une activité de formation en recherche clinique.

\section{Dimension internationale}

«ECRIN/European Clinical Research Infrastructure Network» ERIC, ESFRI Landmark

Coordinateur : Jacques Demotes

Pays partenaires : 23 pays dont 5 membres fondateurs (FR, DE, ES, PT, IT)

Site internet : $\underline{w w w . e c r i n . o r g}$ 


\section{France Life Imaging}

FLI fournit des services aux acteurs du domaine de l'imagerie du vivant (académiques, cliniciens et industriels) et coordonne la recherche dans ce domaine (agents d'imagerie, instrumentation, traitement des données et imagerie interventionnelle). Dotée d'équipements très innovants comme le premier système de Résonance Paramagnétique Electronique humain, le premier système associant tomographie par émission de positons (TEP) et imagerie par ultra-sons (US) ou le premier scanner spectral en France, FLI s'attache à en évaluer l'apport pour la recherche et le soin.

Ces équipements complètent une gamme de 160 systèmes couvrant les modalités d'imagerie (IRM, TEP, US, optique, intravitale, TEP-IRM) dédiés aux études précliniques (57\%) et à la recherche biomédicale et clinique (43\%). Les expertises associées incluent les domaines des neurosciences, du cancer, du cardiométabolisme et de l'infectieux.

Le réseau propose des scenarios de gestion et d'analyse des données adaptés aux besoins de la recherche. II s'attache à promouvoir des solutions pour inter-opérer des bases de données déjà constituées en France.

L'offre de services pour la recherche préclinique et clinique est consolidée par une démarche qualité commune des opérateurs des plateformes et la mise en place de comptes d'exploitation. Enfin, l'expertise des opérateurs est maintenue voire renforcée grâce à la mise en place de nouvelles formations (imagerie moléculaire, US, programmation du logiciel 'paravision®', IRM fonctionnelle (IRMf) et magnétoencéphalographie (MEG) et le soutien à la formation des doctorants et des jeunes chercheurs.

\section{IMPLICATIONS SOCIO-ÉCONOMIQUES}

FLI s'ancre dans l'écosystème national avec:

- la signature d'un accord de coopération en 2015 avec SANOFI;

le rapprochement avec les pôles de compétitivité en santé via MEDICEN pour les manifestations;

- la participation à la création d'un réseau de recherche hospitalière utilisant l'imagerie, FORCEImaging.

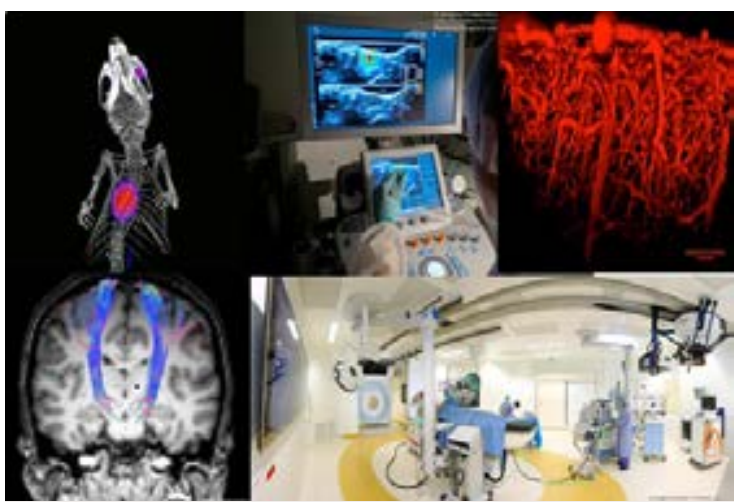

Type de I'infrastructure : IR

\section{Localisation : \\ Orsay}

Localisation des autres sites :

Paris, Bordeaux, Grenoble, Lyon, Marseille

\section{Responsable de l'infrastructure :}

Franck LETHIMONNIER

\section{Création :}

2012

\section{Tutelles / Partenaires :}

CEA, CNRS, INSERM, INRIA, USPC, AMU,

Univ. Bordeaux, UdL, UGA

\section{Contact en France :}

regine.trebossen@cea.fr

\section{www.francelifeimaging.fr}




\section{FRANCE GENOMIQUE}

\section{Infrastructure nationale de génomique et bioinformatique associée}

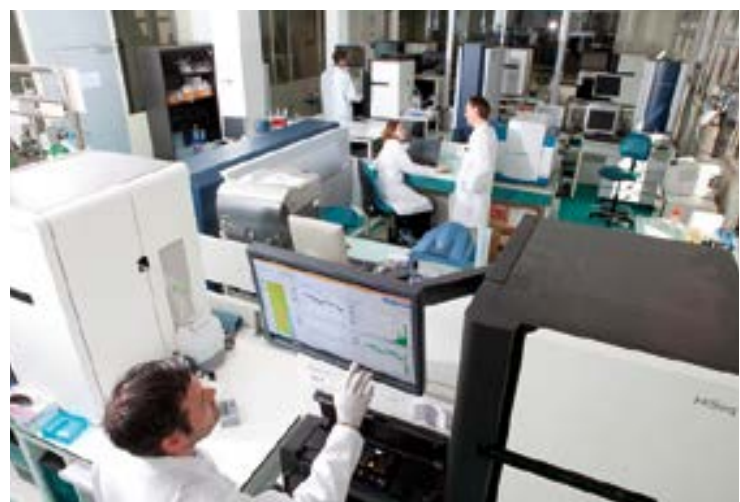

Type de l'infrastructure : IR

\section{Localisation de l'infrastructure :}

Evry

\section{Localisation des autres sites:}

22 plateformes de séquençage, génotypage et/ou bioinformatique

\section{Responsable de l'infrastructure :}

Pierre LE BER

\section{Exploitation :}

2012

\section{Tutelles / Partenaires :}

CEA, CNRS, INRA, Inserm, INRIA, Institut Pasteur, Institut Curie, ENS, Univ. Strasbourg, UdL, Univ. Lille, AMU

\section{Contact en France :}

contact@france-genomique.org

www.france-genomique.org
France Génomique propose à la communauté scientifique publique et privée l'accès aux meilleures plateformes françaises, un accompagnement des projets ainsi que la possibilité de participer à des projets au niveau international.

L'établissement d'une gouvernance et d'un point d'entrée unique pour la gestion des grands et moyens projets ainsi que la mutualisation des équipements et des compétences permet à l'ensemble des scientifiques du domaine de se structurer afin d'atteindre la masse critique nécessaire pour répondre aux besoins croissants de séquençage, de stockage et de traitement des données ainsi que le développement d'outils innovants.

L'infrastructure ainsi créée fournit à la communauté scientifique française publique ou privée:

- une expertise de pointe en génomique et en bio-informatique associée;

- des services compétitifs en génomique et et en bio-informatique en coordination avec IFB.

France Génomique vise ainsi à garantir à la France son indépendance dans des domaines plus que jamais stratégiques pour la recherche en sciences du vivant.

\section{IMPLICATIONSSOCIO-ÉCONOMIQUES}

France Génomique est impliquée dans de grands projets de génomique d'intérêt socio-économique (médecine personnalisée, agronomie...) et dans le développement des technologies de pointe, dans le cadre de programmes de bêta-test (séquençage par nanopores, entre autres)

Forte implication enfin dans le développement de l'utilisation du " big data » pour la génomique, au travers du partenariat avec le centre HPC du CEA/ Teratec (TGCC)

\section{Coût de fonctionnement $11 \mathrm{M} €$}

Coût RH

100 ETPT 


\section{France HADRON}

L'infrastructure nationale, France HADRON (FrHA), coordonne l'ensemble des domaines de la recherche appliquée à l'hadronthérapie proton ou ions légers en France. II s'agit d'une technique avancée de traitement des cancers radiorésistants par radiothérapie. FrHA, organisé en cinq nœuds géographiques, se développe en collaboration étroite avec les centres et réseaux similaires en Europe (ENLIGHT) et dans le Monde (NIRS, NAPTA). Outre l'animation scientifique, elle cofinance l'accès aux faisceaux d'ions des équipes de recherche engagées dans ce domaine. FrHA investit aux cotés des centres cliniques français pour la création de lignes de recherche contribuant au développement des capacités d'accueil scientifique de ces centres.

La recherche en hadronthérapie étant par nature pluridisciplinaire, elle touche aussi bien les champs de la médecine, de la biologie et de la physique des particules. Les travaux de recherche s'articulent stratégiquement autour de quatre programmes de travail (WP) :

- WP1 : Ciblage et évaluation de l'intérêt médical de l'hadronthérapie (Recherche clinique);

- WP2 : Amélioration des plans de traitement (Simulation et modélisation);

- WP3: Amélioration de la compréhension de l'effet des traitements sur les cellules (Radiobiologie):

- WP4 : Amélioration de la qualité des traitements (Instrumentation).

Cette infrastructure positionne la France parmi les pays leader en matière d'hadronthérapie clinique.

\section{IMPLICATIONSSOCIO-ÉCONOMIQUES}

L'hadronthérapie étant une technologie médicale innovante, elle est destinée à avoir un impact sanitaire en termes de guérison de cancers. Dès maintenant, ses programmes de $R \& D$ en instrumentation visent des applications à plus court terme connectées à plusieurs acteurs industriels du domaine, tels que Normandie Hadronthérapie (Caen), Ion Beam Application (Louvain-la-Neuve, Belgique), AIMA (Nice).

\section{DONNÉES}

Estimation du flux de données : environ 0,25 To/an

Stockage : Hébergeurs accrédités en données médicales

Accessibilité : Restreinte à la recherche

Présence dans des réseaux de données : Réseaux nationaux de données brutes et échanges de résultats compilés internationaux

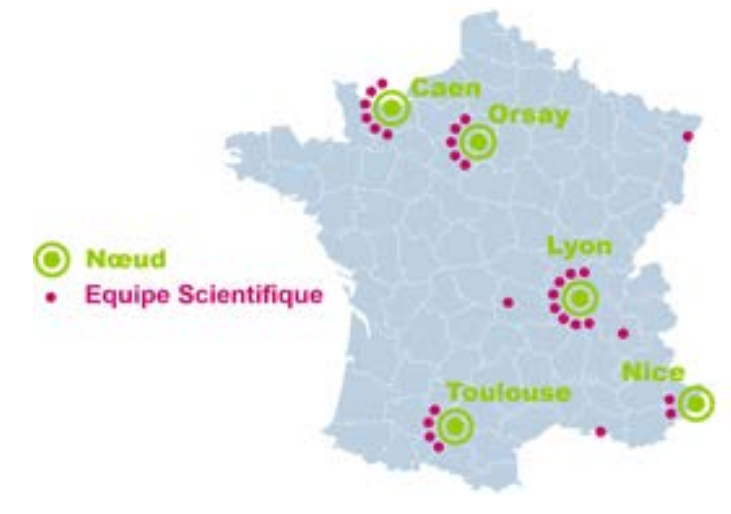

Type de l'infrastructure : IR

\section{Localisation:}

Lyon

Localisation des autres sites:

Caen, Orsay, Toulouse, Nice

Responsable de l'infrastructure :

Jacques BALOSSO

\begin{tabular}{|r|c} 
Création : & Exploitation : \\
\hline 2013 & 2013 \\
\hline
\end{tabular}

\section{Tutelles / Partenaires:}

CNRS, AMU, Association ARCHADE, CAL, CEA, CLB, ECL, Institut Claudius Regaud, Institut Curie, INSERM, INSA, IRSN, UBP, Univ. CAEN, UdL, UNS, Paris VII, Univ. Paris Sud, Univ. Toulouse, Univ. Strasbourg

\section{Contact en France :}

j-c.blouzard@ipnl.in2p3.fr

www.france-hadron.fr 


\section{Infrastructure Française pour la biologie structurale intégrée/ French Infrastructure for Integrated Structural Biology}

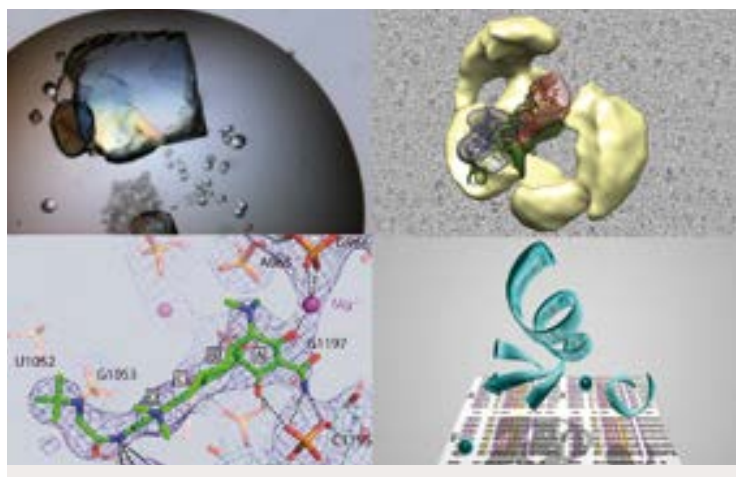

Type de l'infrastructure : IR

Localisation de l'infrastructure:

Illkirch

Localisation des autres sites :

Grenoble, Montpellier, Marseille, Paris

Responsable de l'infrastructure :

Bruno KLAHOLZ

Création: Exploitation:

2011

2012

\section{Tutelles / Partenaires :}

CNRS, CEA, EMBL, Inserm, UGA,

AMU, Univ. Montpellier, Univ. Paris Sud,

Univ. de Strasbourg

\section{Contact en France :}

contact@frisbi.eu

\section{frisbi.eu}

La biologie structurale intégrée permet par la combinaison de différentes approches d'accéder à des informations structurales et dynamiques au niveau de diverses échelles de taille et de temps et ainsi d'améliorer la compréhension du mode d'interaction dynamique des protéines et de leurs complexes fonctionnels, des pathogènes avec leur environnement et ainsi de comprendre les mécanismes qui régissent le fonctionnement des cellules saines et documenter le lien entre dérégulation moléculaire et pathologie.

L'infrastructure Française de Biologie Structurale Intégrée, FRISBI, distribuée sur 5 centres (Strasbourg, Grenoble, Montpellier, Marseille et Paris) offre à la communauté scientifique académique et industrielle, nationale et européenne un accès à un large panel de technologies et de savoir-faire de pointe adaptés à des projets de biologie structurale intégrée (frisbi.eu) dans les domaines de la:

- production d'échantillon en systèmes in vitro, procaryotes et eucaryotes;

- caractérisation biophysique;

- cristallisation:

- cristallographie incluant des liens avec synchrotrons ESRF et SOLEIL:

- microscopie électronique:

- microscopie super-résolutive à fluorescence;

- RMN;

- spectroscopies.

FRISBI a une mission de formation en Biologie Structurale Intégrée qui se réalise à travers l'initiative ReNaFoBis (Réseau National de Formation en Biologie Structurale Intégrative) www.renafobis.fr pour la formation des jeunes chercheurs post master.

\section{IMPLICATIONSSOCIO-ÉCONOMIQUES}

Renforcement du transfert technologique entre la recherche académique et l'industrie pour le développement technologique et pour la biotechnologie. Sept partenariats industriels sont en place au sein de FRISBI dont : automatisation de collecte d'image sur microscopes électroniques, criblage automatique de ligands par cristallographie aux rayons- $\mathrm{X}$, collaboration avec l'industrie en biotechnologie.

\section{Dimension internationale}

Les centres de Strasbourg et Grenoble sont aussi des centres d'Instruct Ltd., ESFRI Landmark

Coordinateur : Dave Stuart,UK

Pays partenaires : BE, CZ, DK, FR, DE, IL, IT,NL, PT, SE, SE, UK

Site internet : www.structuralbiology.eu 


\section{Infrastructure de recherche dédiée aux maladies hautement infectieuses/Highly Infectious Diseases Dedicated Infrastructure ExtensioN}

En raison de leur dangerosité, ces agents pathogènes du groupe de risque 4 doivent être manipulés en laboratoire de niveau de biosécurité 4 (P4), qui ont comme missions la mise en place d'activités de recherche, de diagnostic et de gestion des souches.

Parmi les sept laboratoires NSB4 Européens opérationnels, le laboratoire P4 Inserm Jean Mérieux de Lyon est le seul laboratoire de ce niveau de confinement civil en France. II propose les capacités NSB4 dédiées à la santé humaine les plus importantes, en particulier en ce qui concerne l'expérimentation animale. Il est en effet le seul en Europe à disposer d'une zone d'expérimentation A4 permettant la mise en place de protocoles sur les modèles animaux allant du modèle rongeur jusqu'au primate non humain. Le laboratoire P4 Jean Mérieux, qui est sous l'autorité de I'Inserm depuis 2004, est une grande infrastructure de recherche, ouverte à toute la communauté scientifique nationale et internationale. Cette organisation est unique dans le monde pour ce type d'infrastructure.

Face à l'augmentation du nombre de programmes soumis et l'évolution des normes et réglementations, le projet HIDDEN propose une extension du laboratoire P4 Jean Mérieux existant, qui apportera une amélioration nécessaire des capacités d'expérimentation animale, de diagnostic et de gestion des souches, de la préparation à l'expérimentation sur des bactéries et permettra d'assurer la disponibilité permanente des capacités de niveau de biosécurité 4 .

\section{IMPLICATIONSSOCIO-ÉCONOMIQUES}

L'extension de l'Infrastructure existante permettra d'accroitre l'impact des activités menées dans les secteurs stratégiques visant au développement d'outils thérapeutiques, prophylactiques, de diagnostic, et de sécurité biologique, contribuant ainsi à l'accroissement de la compétitivité française et européenne dans le domaine des maladies infectieuses hautement pathogènes.

\section{Dimension internationale}

Infrastructure pan européenne ERINHA, ESFRI ROadmap : démarrage en 2016.

Coordinateur : Hervé RAOUL

Pays partenaires : FR, DE, PT,UK, SE, EL, BE, RO, IT, SE, HU, AT

Site internet : $\underline{w w w . e r i n h a . e u}$

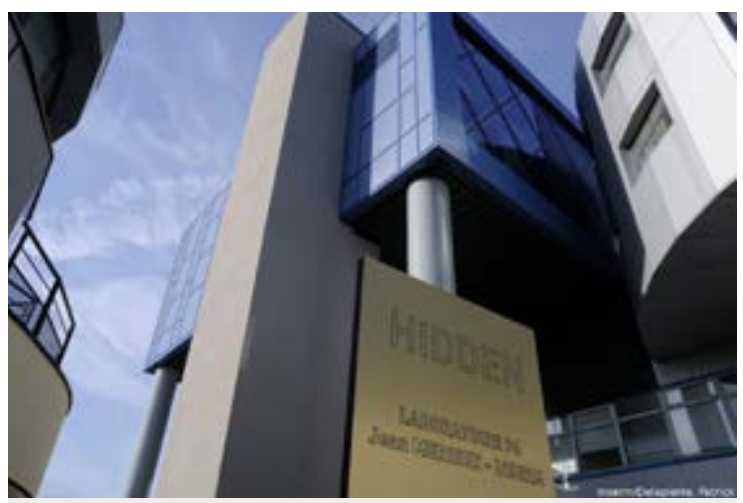

Type de l'infrastructure : IR

\section{Localisation:}

Lyon

Responsable de l'infrastructure: Hervé RAOUL

\section{Création: Exploitation: \\ 2011 2016}

\section{Maintenance :}

Maintenance globale tous les 18 mois

Tutelles / Partenaires :

INSERM, Fondation Mérieux

\section{Contact en France:}

herve.raoul@inserm.fr 


\section{Infrastructure nationale pour la modélisation} des maladies infectieuses humaines et les thérapies innovantes

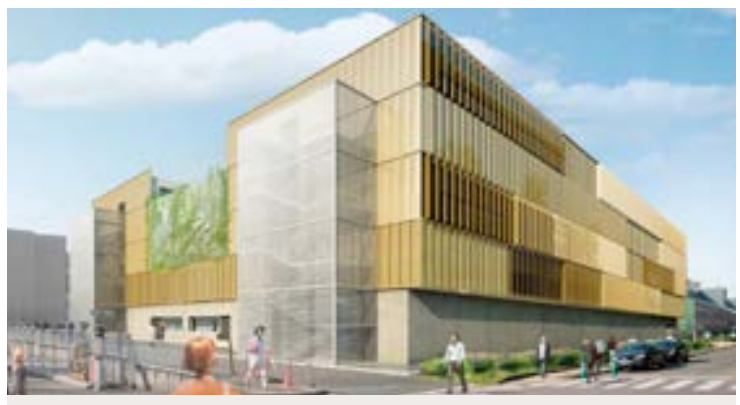

Type de l'infrastructure : IR

Localisation de l'infrastructure:

Fontenay-aux-Roses

Responsable de l'infrastructure/

Représentant en France :

Roger LE GRAND

\section{Création :}

Exploitation :

2015

2015

\section{Exploitation à $100 \%$ de la capacité : 2017}

Jouvence : Equipement TEP-TDM en confinement de type 2 et 3 pour le risque biologique : 2017

Tutelles / Partenaires:

CEA, ANRS, Bertin Pharma, Inserm, Institut Pasteur, Univ. Paris Sud

\section{Contact en France :}

infoidmit@cea.fr

\section{www.idmitcenter.fr}

IDMIT dispose d'une expertise et de compétences reconnues dans le domaine de la physiopathologie, le traitement et la prévention des maladies infectieuses humaines ainsi que les vaccins. IDMIT développe des programmes de recherche sur des pathologies comme la grippe, le Chikungunya, la dengue, la fièvre jaune, les hépatites virales, Ebola, le paludisme, la coqueluche, les chlamydias et la tuberculose.

IDMIT regroupe des plates-formes complémentaires et une expertise dédiées auxétudes pré-cliniques de stratégies préventives et thérapeutiques.

IDMIT dispose de capacités importantes en laboratoires de type $L 2$ et $L 3$ ainsi que des animaleries pouvant héberger des primates non humains $(\mathrm{PNH})$. Ces laboratoires sont équipés pour la chirurgie, l'endoscopie, l'échographie, la radiographie et l'exposition à des pathogènes aériens. Les plates-formes sont spécialisées en biologie moléculaire et cellulaire, immunologie, cytométrie et en imagerie in vivo utilisant les $\mathrm{PNH}$.

En 2017, un nouveau bâtiment donnera accès à de nouveaux laboratoires de niveau 3 et complétera les équipements d'imagerie in vivo par l'implantation d'un Tomographe par Emission de Positons-TomoDensitoMètre (TEP-TDM). Cet ensemble d'équipements en confinement pour le risque biologique est sans équivalent en Europe. Actuellement, IDMIT développe déjà des programmes visant à caractériser de façon dynamique la distribution des médicaments, les réponses immunitaires induites par les vaccins ou à suivre la réplication et la dissémination des pathogènes.

\section{IMPLICATIONSSOCIO-ÉCONOMIQUES}

IDMIT, par son expertise et ses plates-formes technologiques, attire les grandes entreprises pharmaceutiques, les PME nationales et internationales ainsi que des partenaires académiques internationaux, notamment européens. Bertin Pharma, cofondateur et opérateur industriel, concourt à la valorisation des technologies d'IDMIT, par les modèles développés et la mise en place de la norme ISO9001.

\section{Coût de construction \\ $37 \mathrm{M} €$ \\ Coût de fonctionnement : $4 \mathrm{M€}$}




\section{Institut Français de Bioinformatique}

L'IFB est une infrastructure nationale de service en bioinformatique. Sa mission principale est de fournir des ressources de base dans ce domaine à la communauté des sciences du vivant. Les différents services offerts par les plates-formes de I'IFB sont décrits sur le site web de l'infrastructure mais peuvent être regroupés en 5 grandes catégories:

- les données : mise à disposition de collections de données avec une grande valeur ajoutée issue de l'expertise des laboratoires;

- les outils : diffusion d'outils innovants pour analyser les données biologiques produites en laboratoire:

- l'infrastructure : fournir une infrastructure informatique dédiée à la gestion et analyse des données produites par les sciences du vivant. Cela implique non seulement le matériel (CPU, stockage, mémoire, réseau), mais aussi l'accès aux collections de données publiques et aux très nombreux outils utilisés en biologie. Cette infrastructure sera mise à disposition prochainement sous la forme d'un Cloud académique;

- la formation des utilisateurs aux techniques et concepts de la bioinformatique;

- I'appui aux projets de recherche des biologistes en matière d'analyses bioinformatiques.

L'IFB est constituée de 34 plateformes regroupées en 6 pôles régionaux et un nœud national, I'IFB-core, qui est une Unité Mixte de Service dépendante du CNRS, CEA, INRA, INRIA, INSERM.

\section{IMPLICATIONSSOCIO-ÉCONOMIQUES}

ATGC est partenaire de l'entreprise SkulTech.

La plateforme de bioinformatique de l'institut Curie est partie prenante dans l'institut Carnot Curie-Cancer.

ISFinder : Licence d'utilisation de la base de données cédée à DNAStar (USA). URGI : Pôle de compétitivité Céréales Vallées Clermont-Ferrand

\section{DONNÉES}

Estimation du flux de données : Plusieurs Po/an.

Stockage : 2 Po de stockage sur disque et 2 Po de stockage sur bande, celles des PF régionales fournissent 5 Po de stockage.

Accessibilité : IFB gère les données dans les meilleures conditions de sécurité et d'accès.

Présence dans des réseaux de données : L'IFB fait partie d'ELIXIR qui est une infrastructure européenne de bioinformatique distribuée.

\section{Dimension internationale}

L'IFB est le nœud français d'ELIXIR, ESFRI Landmark

Coordinateur : Niklas Blomberg, UK

Pays partenaires : BE, DK, EMBL-EBI, EE, ES, FI, FR, IL, NO, NL, PT, CZ, UK, SE, CH

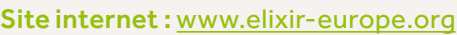

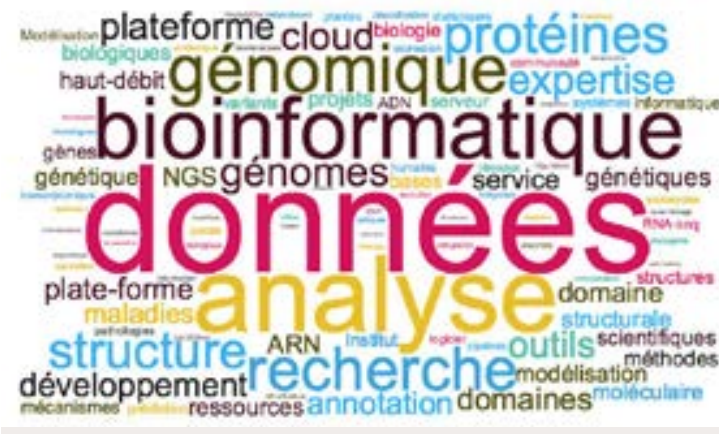

Type de l'infrastructure : IR

Localisation:

Gif-sur-Yvette

Localisation des autres sites:

Distribuée : 34 sites regroupés en 6 pôles régionaux et un nœud national

Responsable de l'infrastructure: Jean-François GIBRAT

\begin{tabular}{|r|c|} 
Création : & Exploitation : \\
\hline 2013 & 2014 \\
\hline
\end{tabular}

Tutelles / Partenaires:

CNRS, CEA, INRA, INRIA, INSERM

Contact en France:

contact@france-bioinformatique.fr

www.france-bioinformatique.fr 


\section{Infrastructure Nationale d'ingénierie des cellules souches et des tissus}

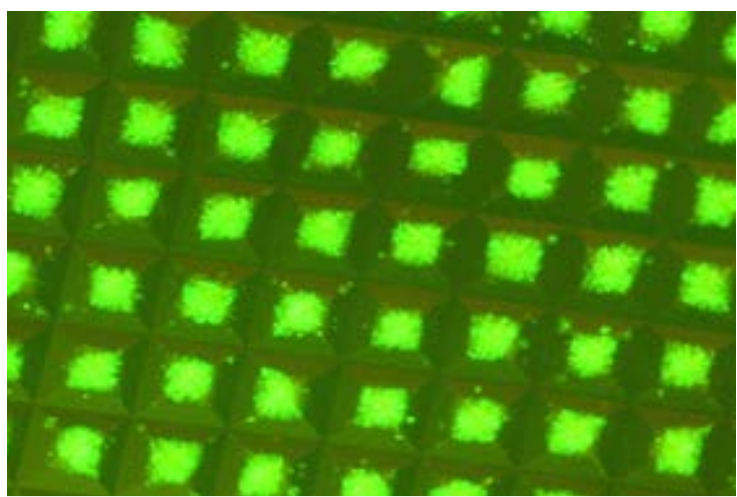

Type de l'infrastructure : IR

Localisation de l'infrastructure :

Villejuif

Localisation des autres sites :

Évry, Strasbourg, Lyon, Montpellier

Responsable de l'infrastructure :

Annelise BENNACEUR GRISCELLI

Création: Exploitation:

2012

2012

\section{Tutelles / Partenaires :}

INSERM, Univ. Paris Sud, Univ. Évry Val d'Essonne, CNRS, Univ. Strasbourg, CHU Montpellier

\section{Contact en France :}

contact@ingestem.fr

\section{www.ingestem.fr}

INGESTEM valide les applications médicales des cellules souches pluripotentes humaines embryonnaires (ESC) et pluripotentes induites (iPSC) avec le support technologique et l'expertise de cinq centres pionniers dans la dérivation des premières cellules ESC humaines en France et leaders dans la reprogrammation cellulaire.

INGESTEM accélère le développement des technologies de pointe et la recherche translationnelle vers les nouvelles thérapies en modélisant les maladies humaines, et la médecine régénératrice à partir des cellules pluripotentes en favorisant les collaborations européennes et internationales. Ouvert aux académiques et industriels, INGESTEM développe les innovations et ressources technologiques dans les stratégies de reprogrammation cellulaire, les protocoles de différenciation et la stratégie de criblages moléculaires, de culture à grande échelle de produits cellulaires de grade clinique.

Les iPSC/ESC sont utilisées pour l'ingénierie du génome et la génération d'organoïdes pour des thérapies innovantes et thérapies cellulaires. Les cellules iPSC/ESC dérivées de modèles animaux sont disponibles pour évaluer l'innocuité et l'efficacité thérapeutique in vivo des greffons cellulaires dérivés des ESC/iPSC.

INGESTEM rassemble des plateformes de culture de cellules souches, de reprogrammation et d'ingénierie cellulaire, de génomique fonctionnelle et d'édition du génome, de transgénèse dans les modèles primates, de criblage moléculaire et d'usines cellulaires automatisées.

\section{IMPLICATIONSSOCIO-ÉCONOMIQUES}

Modèles pathologiques d'IPS/ES en lien avec les hôpitaux universitaires pour la recherche et programmes européens.

Haplobanque iPS grade clinique en lien avec GAIT (réseau international). Brevets en thérapie cellulaire.

Découverte de nouveaux médicaments par criblage de modèles cellulaires d'IPS de maladies génétiques.

Création de trois start-ups et d'une PME.

Partenariats avec 2 entreprises pharmaceutiques.

\section{DONNÉES}

Présence dans des réseaux de données : Registre Européen des cellules souches pluripotentes

\section{Dimension internationale \\ Consortium International : GAIT « Global Alliance iPS Therapies » \\ Coordinateur : Marc Turner, UK \\ Pays partenaires: USA, UK, CA, FR, KR, JP, AU, AR}




\section{Infrastructure française distribuée pour la métabolomique dédiée à l'innovation, à la formation et au transfert de technologie}

MetaboHUB est une fédération de 4 plateformes labellisées IBiSA, certifiées ISO 9001 sous la tutelle administrative de I'INRA et du CEA :

1. Bordeaux (INRA, CNRS et Univ. Bordeaux);

2. Clermont-Ferrand (INRA, CNRS, Univ. Blaise Pascal)

3. Toulouse, (Univ. Paul Sabatier, INSA, INRA, CNRS, INSERM);

4. Paris-Saclay, (CEA, CNRS, Univ. Paris XI).

Les plateformes de Bordeaux, Toulouse et Clermont sont stratégiques pour I'INRA.

L'objectif de MetaboHUB est de construire une infrastructure nationale ouverte et d'intégrer le réseau des grandes infrastructures européennes.

De 2013 à 2016, MetaboHUB développe des outils, des services web et des bases de données nécessaires à la métabolomique et à la fluxomique. A partir de 2017, I'infrastructure fournira aux partenaires académiques et privés les méthodes d'analyse standardisées (site web), une base de données spectrales (PeakForest) intégrée à un écosystème d'outils pour l'exploration des données (W4M) et les moyens informatiques pour reconstruire les réseaux métaboliques (MetExplore).

Les technologies développées (RMN, LC-MS, GC-MS, statistique, bioinformatique) permettent le phénotypage biochimique à haut débit de grands ensembles d'échantillons et l'analyse du métabolome (liquides biologiques de cohortes humaines, extraits de plantes, d'animaux, de micro-organismes, etc.) et les mesures de flux métaboliques à haut débit chez les cellules procaryotes et eucaryotes.

\section{IMPLICATIONS SOCIO-ÉCONOMIQUES}

MetaboHUB est au service des laboratoires publics et privés dans les domaines de la santé humaine, la nutrition, la production alimentaire, la toxicologie, l'agriculture, la chimie verte, l'environnement et les biotechnologies. MetaboHUB partage tous ses savoir-faire et ses outils avec les différentes communautés scientifiques françaises.

\section{DONNÉES}

Estimation du flux de données : A court terme : 8.2 To, à moyen terme : 18 To

Stockage : Les données brutes (spectres de masse et RMN) sont stockées au niveau de chaque plateforme (volume allant de 10 To à 40 To par PF). Lorsque ces données ne sont plus confidentielles, elles sont déposées dans l'entrepôt européen MetaboLights.

Accessibilité : données accessibles dans le cadre de l'accord de consortium.

MetaboHUB va fournir un cadre commun d'outils bio-informatiques pour la gestion et l'analyse des données de métabolomique et fluxomique : "Workflow4Metabolomics.org", interface avec " MetExplore » et mise en place des bases de données spectrales "PeakForest " ou des bases de données de ce type. Un service pour accéder aux données et/ou les exploiter est prévu. Une négociation est en cours pour accéder aux serveurs de l'Institut Français de Bioinformatique (IFB) pour pouvoir stocker les données.

Présence dans des réseaux de données : La ressource de calcul Workflow4Metabolomics est développée conjointement par MetaboHUB et I'IFB et hébergée par ce dernier (plateforme ABiMS, Roscoff).

Ouverture des "data center » INRA de Toulouse pour la centralisation, le stockage et la sauvegarde des données et l'hébergement de ressources de calculs (serveurs applicatifs, serveurs web et serveurs de bases de données).

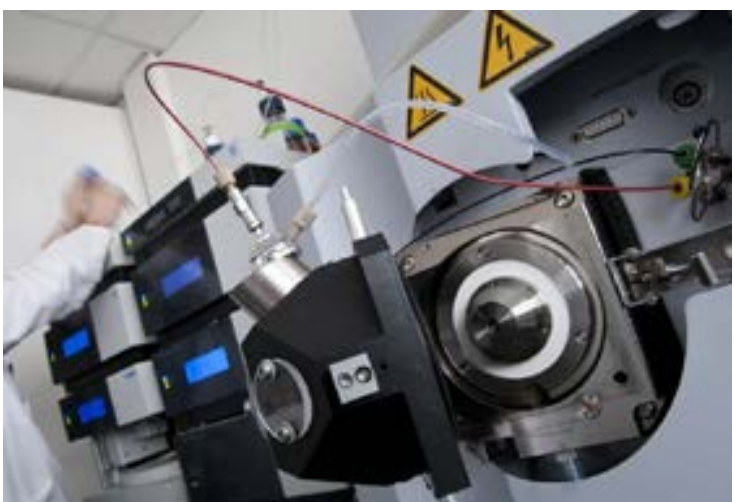

Type de I'infrastructure : IR

\section{Localisation :}

Bordeaux

\section{Localisation des autres sites :}

Clermont-Ferrand, Toulouse, Saclay

Responsable de l'infrastructure :

Dominique ROLIN

\begin{tabular}{|r|c|} 
Création : & Exploitation : \\
\hline 2013 & 2017 \\
\hline
\end{tabular}

\section{Tutelles / Partenaires :}

INRA, CEA, CNRS, INSERM, INSA Toulouse, UPB, Univ. Bordeaux, UPS, UPMC

\section{Contact en France:}

contact@metabohub.fr

\section{www.metabohub.fr}




\section{Infrastructure de Recherche translationnelle pour les biothérapies en neurosciences}

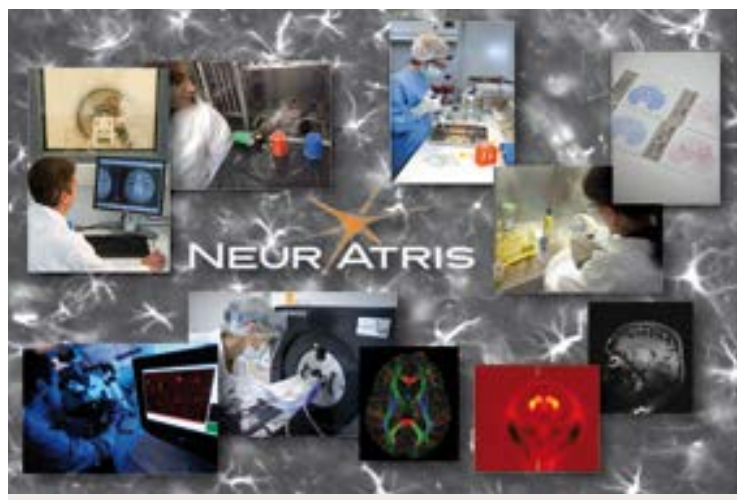

Type de l'infrastructure : IR

\section{Localisation de l'infrastructure :}

Fontenay-aux-Roses

\section{Localisation des autres sites :}

Paris, Gif-sur-Yvette, Orsay, Evry, Créteil

Responsable de l'infrastructure :

Philippe HANTRAYE

Création: Exploitation:

2012

2012

\section{Tutelles / Partenaires :}

CEA, IHU-A-ICM, Inserm, Inra, UPEC

\section{Contact en France :}

Philippe.hantraye@cea.fr

\section{www.neuratris.com}

Se fondant sur cinq centres de recherche principaux, tous situés dans la région parisienne, NeurATRIS représente l'une des plus grande concentration de chercheurs en neurologie en Europe. Regroupant dans un institut sans murs, I'Institut d'imagerie biomédicale du CEA, I'IHU de Neurosciences Translationnelles de Paris, le consortium de BIRD, les hôpitaux Henri Mondor et Bicêtre, avec des compétences et une expertise dans les biothérapies et les troubles du développement neurologique, NeurATRIS se positionne comme la plus grande infrastructure européenne qui conduit des projets de R \& D et qui fournit des services aux universitaires, aux cliniciens et aux industriels en neurosciences.

Partenariats public/privé solides et durables le long de trois axes:

1. développer des lignes spécifiques de recherche translationnelle et des savoir-faire en neurosciences visant à découvrir et à qualifier au niveau préclinique et clinique de nouvelles solutions thérapeutiques pour les maladies neurodégénératives;

2. surmonter la fragmentation en réunissant différents spécialistes de la cellule, du gène et des technologies basées sur les médicaments permettant la fusion trans-fonctionnelle des compétences et de l'expertise dans une structure unique;

3. faciliter l'accès à des plates-formes de haut niveau (avec accès unique et personnel hautement qualifié). Les projets externes transitent dans les différents centres - en fonction de leur stade de développement et de l'expertise requise - sous la supervision d'un gestionnaire de projet en recherche translationnelle.

\section{IMPLICATIONSSOCIO-ÉCONOMIQUES}

Deux approches complémentaires : 1) la recherche pour l'innovation à travers des thérapies cellulaires, génétiques ou médicamenteuses et l'évaluation fonctionnelle in vivo. 2 ) une gamme de services de recherche avec des plates-formes de haute technologie en préclinique et en clinique, et une expertise couvrant tout le continuum translationnel en neurosciences et neurologie. Principaux partenaires industriels (2014) : Oxford Biomedica Ltd, BrainGene, Novartis et Roche

\section{Dimension internationale \\ EATRIS ERIC, ESFRI Landmark \\ Coordinateur : Anton Ussi, NL \\ Pays partenaires : NL, IT, ES, FI, CZ, FR, EE \\ Site internet : $\underline{w w w . e a t r i s . e u}$}




\section{Consortium Préindustriel des vecteurs de Thérapie Génique}

Le consortium PGT a pour ambition de jouer un rôle central au plan international pour la production des vecteurs de thérapie génique indispensables à la réalisation des essais cliniques, de plus en plus nombreux dans le domaine des biothérapies. Le rôle du démonstrateur est de valider des procédés de bioproduction à une échelle préindustrielle, prérequis à l'industrialisation complète des procédés de fabrication des produits de thérapie génique et à l'émergence d'une véritable filière industrielle des médicaments de thérapie génique.

Son objectif est double : accélérer le développement des procédés de production de vecteurs aux normes pharmaceutiques et répondre à la demande croissante de vecteurs thérapeutiques indispensables aux essais cliniques pour les maladies rares, qui se multiplient dans le monde.

Dans ce cadre, l'action du Démonstrateur PGT est basée sur des applications de preuve de concept : études réglementaires précliniques et essais cliniques de phases I/II.

Le consortium se donne pour mission de délivrer des produits de thérapie génique pour des essais de phase I/II à des investigateurs académiques ou industriels.

Le consortium réunit quatre acteurs de premier plan dans le domaine de la thérapie génique et de la bioproduction en France: Généthon et GenoSafe localisés à Evry et d'autre part Atlantic BIO GMP (ABG-EFS) et le Centre Hospitalo-Universitaire (CHU), tous deux localisés à Nantes.

\section{IMPLICATIONSSOCIO-ÉCONOMIQUES}

Depuis sa création, PGT a produit pour 11 utilisateurs externes (académiques, Biotechs ou société Pharmaceutique), localisés en France ou à l'international, qui développent des médicaments de thérapie innovante. Cela contribue à la création d'emplois et au maintien de la position de leader de la France dans le domaine de la bioproduction de vecteurs de thérapie génique.

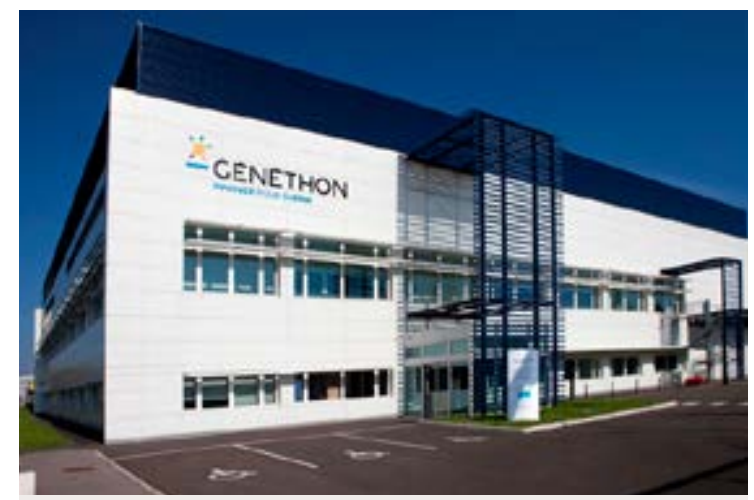

Type de l'infrastructure : IR

\section{Localisation:}

Evry

\section{Localisation des autres sites :}

Saint-Herblain

\section{Responsable de l'infrastructure :}

Frédéric REVAH

\section{Création :}

2011

\section{Tutelles / Partenaires :}

Généthon, GenoSafe, Atlantic BIO GMP

(ABG-EFS), CHU Nantes

\section{Contact en France :}

frevah@genethon.fr

\section{www.pgt-consortium.fr}




\section{Infrastructure Française de protéomique/ Proteomics French Infrastructure}

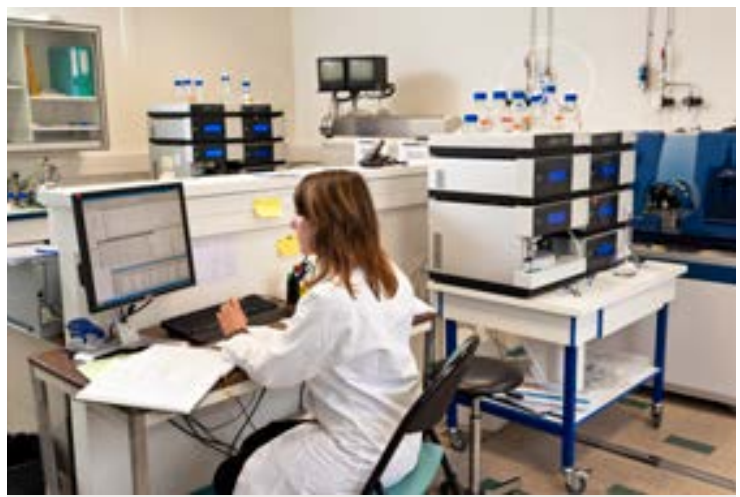

Type de l'infrastructure : IR

\section{Localisation de l'infrastructure:}

Grenoble

\section{Localisation des autres sites :}

Toulouse, Strasbourg

\section{Responsable de l'infrastructure :} Jérôme GARIN

\section{Création :}

2012

\section{Tutelles / Partenaires :}

CNRS, CEA, INSERM, UGA, UPS,

Univ. Strasbourg

\section{Contact en France :}

jerome.garin@cea.fr

\section{www.profiproteomics.fr}

ProFl est l'infrastructure nationale qui réunit les trois laboratoires français leaders dans le domaine de la Protéomique. Les développements technologiques et méthodologiques réalisés dans le cadre de cette infrastructure ont pour objectif de permettre la détection et la quantification de protéines présentes au sein d'échantillons biologiques complexes, l'étude de leur dynamique ainsi que de leurs modifications post-traductionnelles. Les applications se trouvent à la fois dans le domaine de l'étude fine des mécanismes moléculaires mis en jeu par les grandes fonctions cellulaires, et dans le domaine de la découverte et de l'évaluation de nouveaux biomarqueurs de pathologies. La maitrise des approches les plus pointues permet à ProFI de répondre efficacement aux nombreuses demandes issues de collaborateurs académiques et industriels. ProFI mène également une action de formation destinée à irriguer l'ensemble de la communauté protéomique française; par exemple pour permettre aux opérateurs des plateformes protéomiques de prendre en main l'environnement logiciel qui a été développé parl'infrastructure.

\section{IMPLICATIONSSOCIO-ÉCONOMIQUES}

Des partenariats ont été établis entre l'infrastructure ProFl et les sociétés Transgene, Pierre Fabre, Sanofi Pasteur, Servier, L'Oréal, Genticell, Agronutrition, Virbac et Novalix.

Les principaux domaines traités sont le contrôle qualité d'échantillons protéiques ou cellulaires et la caractérisation fine de protéines, en particulier d'anticorps monoclonaux. 


\section{Industrial Biotechnology Innovation and Synthetic Biology Accelerator}

IBISBA-FR conduit des projets de R\&D finalisés (TRL3-5) et soutient ainsi l'émergence de la biotechnologie industrielle pour la valorisation du carbone renouvelable et donc la bioéconomie.

IBISBA-FR, implanté en lle de France et à Toulouse, comporte plusieurs plateformes technologiques dédiées aux travaux bio-informatiques, à la construction à haut débit de souches, aux méthodes analytiques, aux opérations unitaires et aux bioconversions/fermentations, ainsi que deux autres plateformes dédiées à l'évaluation d'impacts environnementaux et à l'éthique pratique. La mission d'IBISBA-FR est de traduire des résultats de recherche en outils et procédés pour l'industrie. Pour mener à bien cette mission, IBISBA-FR

1. assure la promotion de la biotechnologie industrielle pour l'utilisation du carbone renouvelable;

2. catalyse l'innovation scientifique en biotechnologie industrielle;

3. crée un continuum entre la recherche et l'implémentation des biotechs en milieu industriel.

Une caractéristique d'IBIBSA-FR est le partenariat public-privé de TWB composé de 23 entreprises.

IBISBA-FR conduit plusieurs types de projets R\&D

- précompétitifs, autofinancés par IBISBA-FR (génère de la Plauxchercheurs publics, bases pour la création de start-ups);

compétitifs taillés aux besoins d'entreprises souhaitant accélérer le développement de leurs projets en biotechnologie:

- intermédiaires cofinancés par des agences publiques (ex: ANR et H2020) et le secteur privé.

\section{IMPLICATIONSSOCIO-ÉCONOMIQUES}

A travers TWB, IBISBA-FR a déjà enregistré une activité conséquente : 55 projets lancés fin 2015, $18 \mathrm{M} €$ de contrats, 11 brevets déposés. Création d'une start-up, EnobraQ, résultat d'un projet précompétitif (Carboyeast) et créée en partie grâce à un financement obtenu du fond d'investissement Sofinnova.

\section{Dimension internationale \\ EU-IBISBA projet en construction \\ Coordinateur : Michael O'Donohue}

Pays partenaires : FI, IT, ES, EL, SE, UK, NL, PO, BE, DE

Site internet : $w w w . i b i s b a . c o m$

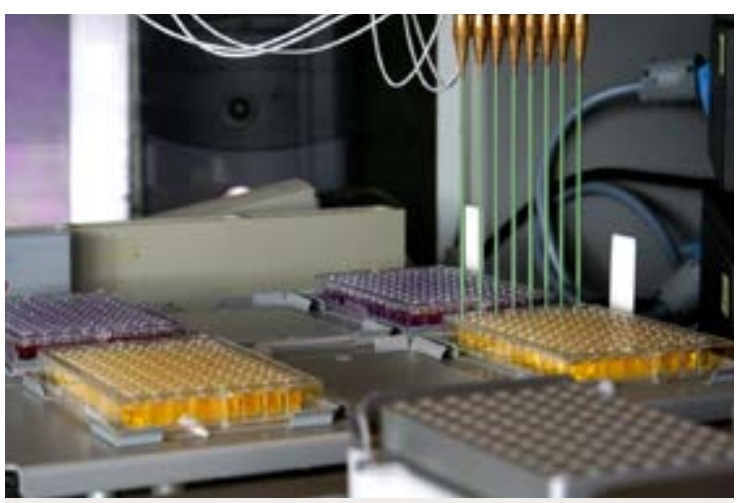

Type de l'infrastructure : Projet

\section{Statut de l'infrastructure :}

Unité mixte de service

\section{Localisation :}

Toulouse

Localisation des autres sites :

Région lle-de-France

Responsable de l'infrastructure: Michael O'DONOHUE

\section{Création :}

Exploitation :

2010

Tutelles / Partenaires :

CNRS, CEA, INRA, INSA

\section{Contact en France :}

michael.odonohue@insa-toulouse.fr 


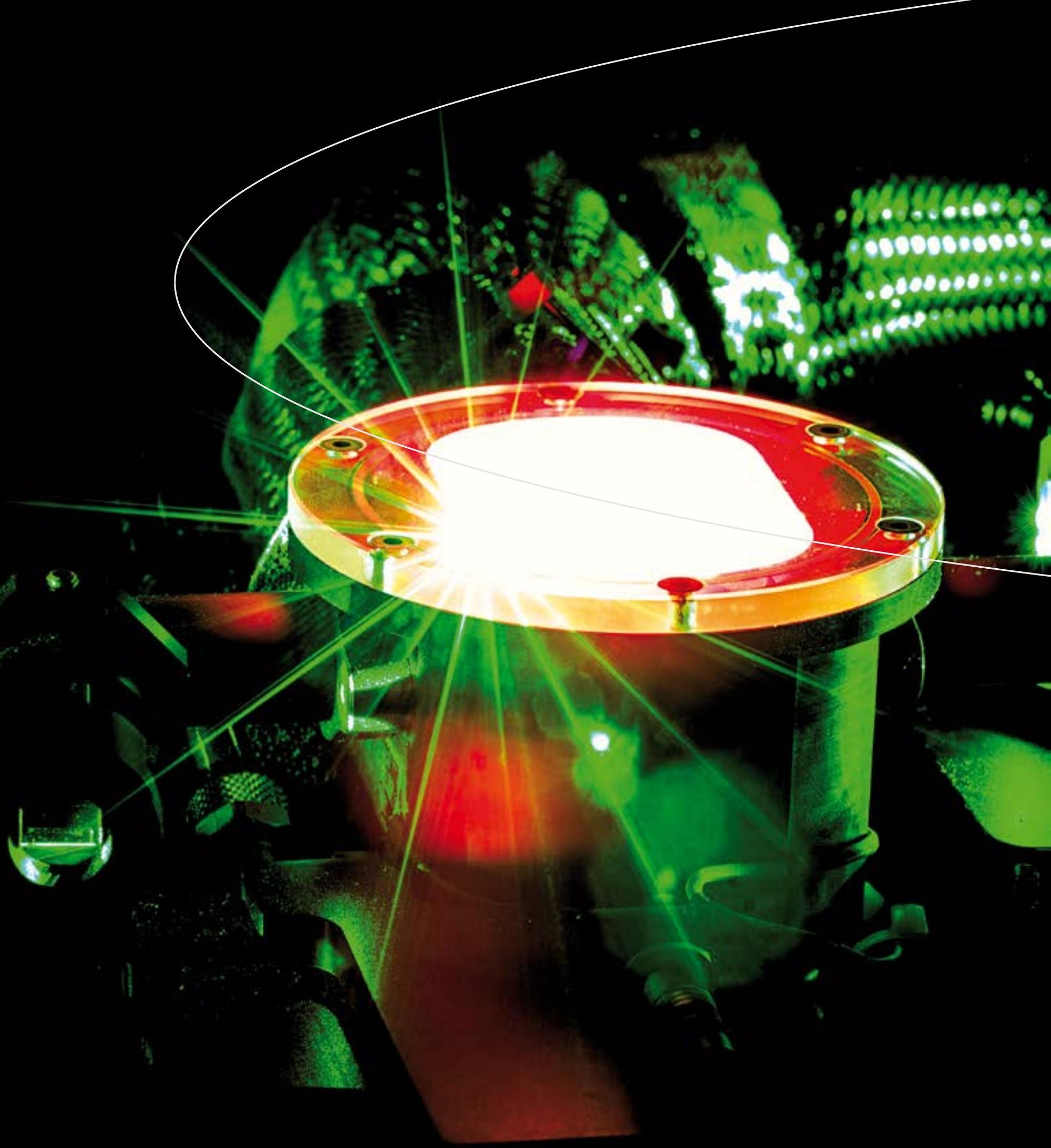




\section{SCIENCES DE LA MATIÈRE ET INGÉNIERIE}

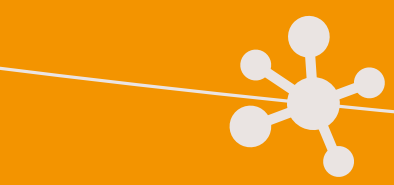




\section{Sciences de la matière}

\section{et ingénierie}

Les infrastructures de recherche consacrées aux sciences de la matière et à l'ingénierie, centres de rayonnement synchrotron (ESRF, SOLEIL), centres de diffusion neutronique (ILL, LLB-ORPHEE), centres de lasers intenses (LULI-APOLLON et PETAL) en France ainsi que les projets européens, de source intense pulsée (XFEL à Hambourg), de source neutronique à spallation (ESS à Lund), le centre de champs magnétiques intenses (LNCMI/EMFL) mais aussi les réseaux de plateformes (EMIR, RENARD, RENATECH, METSA, RMN-THC, FT-ICR) se caractérisent par I'utilisation de sondes de différentes natures : des rayonnements couvrant le plus large spectre électromagnétique, des ondes millimétriques au rayons $X$ très durs, voire aux rayons gamma; les photons délivrés par des lasers de Ultra Haute Intensité (UHI) ou de Haute Densité d'Energie (HDE); les neutrons, avec une forte capacité de pénétration dans la matière; les champs magnétiques intenses.

Ces infrastructures sont des lieux où se créent des synergies fortes entre ingénieurs, techniciens, physiciens " machine » d'une part et scientifiques utilisateurs de différentes disciplines. Elles permettent d'offrir aujourd'hui des outils de recherche sans cesse optimisés et modernisés, qui répondent aux attentes des communautés scientifiques. Le Comité de coordination thématique sur les très grandes infrastructures de recherche (TGIR) constitué du CEA et du CNRS veille à maintenir la diversité et la pluridisciplinarité de ces infrastructures en accord avec la Stratégie nationale de recherche et d'innovation.
L'accès combiné, souvent de façon complémentaire, aux diverses infrastructures, permet aux scientifiques d'obtenir des avancées uniques dans le développement de la connaissance de la matière sous toutes ses formes (gaz, liquide, solide, plasma) et en reproduisant en laboratoire toutes les conditions extrêmes (très hautes pressions, hautes et très basses températures, hauts champs magnétiques). Tous les domaines scientifiques sont concernés : physique, chimie, astrophysique, géologie, biologie, archéologie, paléontologie et patrimoine culturel. Ainsi, ces infrastructures contribuent à répondre aux questions scientifiques les plus fondamentales tout en participant aux avancées dans des domaines plus appliqués tels que l'énergie ou la santé. En permettant de caractériser les matériaux, elles contribuent, en particulier, au développement de la nanoélectronique et des nanosciences.

Le développement des technologies à des échelles nanométriques a conduit les ingénieurs dans plusieurs secteurs clés de l'économie à façonner la matière et à l'observer en utilisant des outils de synthèse et d'observation jusqu'alors presqu'exclusivement utilisés par les chercheurs. Cette tendance augmente sensiblement aujourd'hui et devrait s'accentuer dans les années à venir conférant aux sites équipés de grandes infrastructures un pouvoir d'attractivité supplémentaire pour les développements industriels. 
Q. LISTE DES INFRASTRUCTURES DE RECHERCHE

o DANS LE DOMAINE SCIENCES DE LA MATIÈRE ET INGÉNIERIE

\begin{tabular}{|c|c|c|c|}
\hline CATÉCORIE & NOM & NOM COMPLET & ESFRI \\
\hline TGIR & ESRF & $\begin{array}{l}\text { Source Européenne de Rayonnement } \\
\text { Synchrotron/ } \\
\text { European Synchrotron Radiation Facility }\end{array}$ & $\begin{array}{l}\text { ESRF Upgrade } \\
\text { Ph } 1 \text { (2006) } \\
\text { ESRF Upgrade } \\
\text { Ph } 2 \text { (2016) }\end{array}$ \\
\hline TGIR & ESS & European Spallation Source & ESS (2006) \\
\hline TGIR & ILL & Institut Max von Laue - Paul Langevin & $\begin{array}{l}\text { ILL Upgrade Ph } 1 \\
\text { (2006) }\end{array}$ \\
\hline TGIR & Orphée/LLB & ORPHEE/Laboratoire Léon Brillouin & \\
\hline TGIR & Soleil & $\begin{array}{l}\text { Source Optimisée de Lumière d'Energie } \\
\text { Intermédiaire du LURE }\end{array}$ & \\
\hline TGIR & XFEL & European X-ray Free Electron Laser & XFEL (2006) \\
\hline IR & EMIR & $\begin{array}{l}\text { Fédération des Accélérateurs pour les Études } \\
\text { des Matériaux sous Irradiation }\end{array}$ & \\
\hline IR & FT-ICR & $\begin{array}{l}\text { Réseau national de spectrométrie de masse FT-ICR } \\
\text { à très haut champ }\end{array}$ & \\
\hline IR & LNCMI & $\begin{array}{l}\text { Laboratoire National des Champs Magnétiques } \\
\text { Intenses }\end{array}$ & EMFL (2008) \\
\hline IR & LULI-APOLLON & Laboratoire d'Utilisation des Lasers Intenses & \\
\hline IR & METSA & $\begin{array}{l}\text { Microscopie Electronique en Transmission } \\
\text { et Sonde Atomique }\end{array}$ & \\
\hline IR & PETAL & PETAwatt Aquitaine Laser & \\
\hline IR & RMN & $\begin{array}{l}\text { Résonance Magnétique Nucléaire } \\
\text { à Très Hauts Champs }\end{array}$ & \\
\hline IR & Renard & REseau NAtional de Rpe interDisciplinaire & \\
\hline IR & RENATECH & $\begin{array}{l}\text { Réseau NAtional des grandes centrales } \\
\text { de TECHnologies }\end{array}$ & \\
\hline Projet & $E R / H S-F R^{1}$ & $\begin{array}{l}\text { European Research Infrastructure } \\
\text { for Heritage Science }\end{array}$ & ERIHS (2016) \\
\hline
\end{tabular}

1 Relève également du secteur «Sciences Humaines et Sociales», dans lequel la fiche de l'infrastructure est présentée. 


\section{Source Européenne de Rayonnement Synchrotron/ European Synchrotron Radiation Facility}

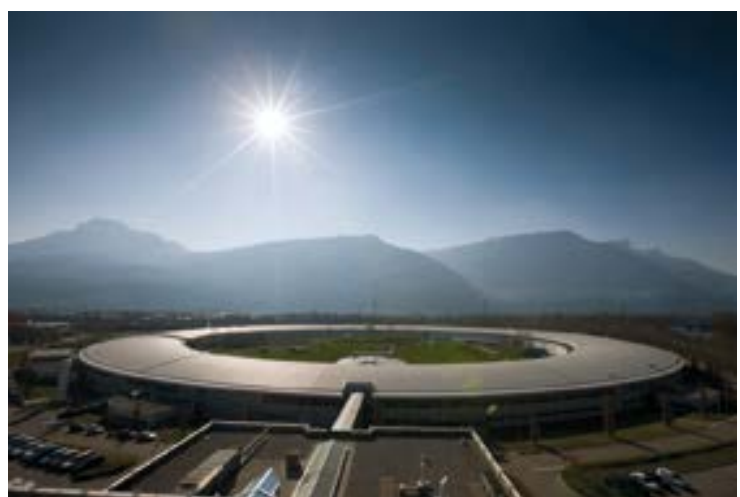

Type de l'infrastructure : TGIR

Statut juridique de l'infrastructure:

Société civile de droit français

Localisation de l'infrastructure:

Grenoble

Représentantes de la France :

Amina TALEB-IBRAHIMI, Maria FAURY

\section{Création :}

Exploitation :

1988

1994

Jouvence : 2009-2015 Upgrade Phase I 2015-2022 ESRF-Extremely Brilliant Source, ESRF-EBS

\section{Tutelles / Partenaires : \\ CNRS, CEA}

\section{Contact en France :}

amina.taleb@cnrs-dir.fr

maria.faury@cea.fr

\section{www.esrf.eu}

L'ESRF est la source européenne de rayonnement synchrotron produisant des rayons $X$ à haute énergie (très durs de 10 à $300 \mathrm{keV}$ ) qui sont distribués sur 43 stations d'expériences (lignes de lumière) mises à la disposition de la communauté scientifique et qui bénéficient d'une instrumentation de pointe. L'ESRF a aussi pour mission d'utiliser son expertise scientifique afin de développer de nouvelles méthodes et technologies. Les domaines d'application de ces expériences, sont extrêmement vastes : les sciences du vivant, la biologie et les applications médicales, les sciences de la matière molle, la chimie et la physique des matériaux, les sciences de l'environnement et le patrimoine culturel. L'ESRF est le leader incontesté des centres de rayonnement synchrotron au niveau européen et même mondial. Le projet d'extension le «ESRF Upgrade Programme » voit sa première phase (2009-2015) se terminer avec l'amélioration des performances de l'accélérateur et du faisceau d'électrons ainsi que la création de 8 lignes de lumière de nouvelle génération soit 19 stations expérimentales. La phase 2, ESRF-EBS (2015-2022), consiste à modifier intégralement l'anneau de stockage permettant une augmentation de la brillance d'un facteur 100 Des programmes similaires au projet ESRF-EBS sont aussi dans un état d'étude avancé aux États-Unis, au Japon et en Chine.

\section{IMPLICATIONS SOCIO-ÉCONOMIQUES}

Les partenariats avec l'industrie interviennent à différents niveaux : mesures : contrats avec les industriels (2.2M€ de revenus en 2014); en développement et conception conjointe de dispositifs innovants (30 licences de transfert de technologies);

en retombées économiques : depuis 1988, plus de 2 Md€ reversés vers le secteur commercial et industriel.

\section{DONNÉES}

Estimation du flux de données : 2 Po/an

Stockage : Stockage à l'ESRF

Présence dans des réseaux de données : projets FP7 et $\mathrm{H} 2020$ pour améliorer les aspects liés à la propriété, le stockage, l'archivage, le traitement et l'accès.

\section{Coût de construction \\ - \\ 2598 MF (1987) + ESRF II \\ UP Phase $1=168 \mathrm{M} €$ \\ + ESRF-EBS =149,1 M€ \\ Coût de fonctionnement : \\ $100 \mathrm{M€}$ dont $27,5 \mathrm{M€}$ \\ de contribution française}

Coût RH

597,5 ETPT

\section{Dimension internationale \\ ESRF-Extremely Brilliant Source, ESFRI Landmark \\ Coordinateur : Francesco Sette, Directeur Général \\ Pays partenaires : FR (27,5\%), DE (24\%), IT (13,2\%), UK (10,5\%), SE (4\%), $\mathrm{CH}(4 \%), \mathrm{RU}(6 \%)$, Benesync BE, NL (5,8\%), Nordsync DK, FI, NO, SE (5\%) 8 Associés : IL (1,5\%), PL (1\%), PT (1\%), AT (1,3\%), CentralSync HU, CZ, SK $(1,05 \%)$, ZA $(0,3 \%)$}




\section{European Spallation Source}

La source européenne de spallation ESS sera une infrastructure de recherche pluridisciplinaire utilisant la source de neutrons la plus puissante au monde pour explorer la matière dans de nombreux domaines, des sciences de la vie à l'ingénierie des matériaux, de la conservation du patrimoine au magnétisme. ESS sera environ 30 fois plus performante que les installations existantes et ouvrira aux scientifiques de nouvelles possibilités de recherche dans les domaines du magnétisme, de la spectroscopie à ultra-haute résolution $\left(10^{-8} \mathrm{eV}\right)$, de la physique des particules grâce à I'utilisation de neutrons ultra-froids, mais également dans les domaines plus appliqués de la santé, de l'environnement, de l'énergie, du climat, des transports, de la pharmacie.

ESS se compose d'un accélérateur linéaire de 600 m de long qui confère une énergie de $2,5 \mathrm{GeV}$ à des protons impactant une cible de tungstène avec une puissance faisceau de $5 \mathrm{MW}$. Cette source produira des pulses longs de neutrons $(2.86 \mathrm{~ms}$ à une fréquence de $14 \mathrm{~Hz}$ ) correspondant à un pic de flux de neutrons 30 fois supérieur à celui de la source à spallation américaine SNS (impulsions courtes).

ESS devrait produire ses premiers neutrons fin 2019, pour une exploitation à pleine puissance en 2023-2025 avec 16 spectromètres.

\section{IMPLICATIONSSOCIO-ÉCONOMIQUES}

Création autour de l'ESS et du synchrotron MaxIV, d'un quartier regroupant des installations de recherche, universitaires, ou des entreprises en lien avec l'innovation et la recherche.

\section{DONNÉES}

Estimation du flux de données : 2 Po/an

Stockage : DMSC : Data Management and Software Center situé à Copenhague

\section{Coût de construction}

1843 M€ dont 147,5 M€

de contribution française

Coût de fonctionnement :

120 M€ dont 10 à $12 \mathrm{M€}$

de contribution française

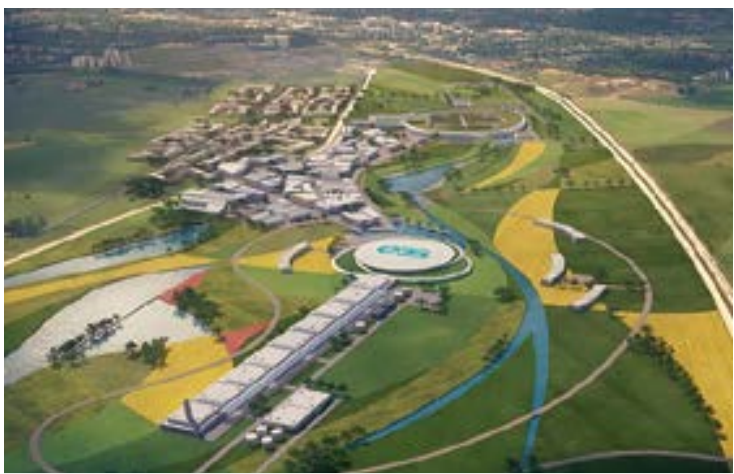

Type de l'infrastructure: TGIR

\section{Statut de l'infrastructure :}

ERIC

\section{Localisation:}

Lund, SE

\section{Représentantes de la France :}

Patricia ROUSSEL CHOMAZ,

Amina TALEB-IBRAHIMI

\section{Création: Exploitation: \\ 2014 \\ $2023-2025$}

Tutelles / Partenaires :

CNRS, CEA

\section{Contact en France :}

patricia.roussel-chomaz@cea.fr

amina.taleb@cnrs-dir.fr

\section{europeanspallationsource.se}

\section{Dimension internationale \\ ESS ERIC, ESFRI Landmark \\ Coordinateur : Jim Yek, Directeur Général \\ Pays partenaires : SE (35\%) , DK (12,5\%), DE (10\%),UK (9,8\%), FR ( $8 \%)$, IT (5,66\%), ES (4,73\%), CH (3,5\%), NO (2,5\%),PL (2\%), HU (0,95\%), \\ CZ $(2 \%)$, EE $(0,25 \%)$, LT $(0,45 \%)$}


Institut Max von Laue - Paul Langevin

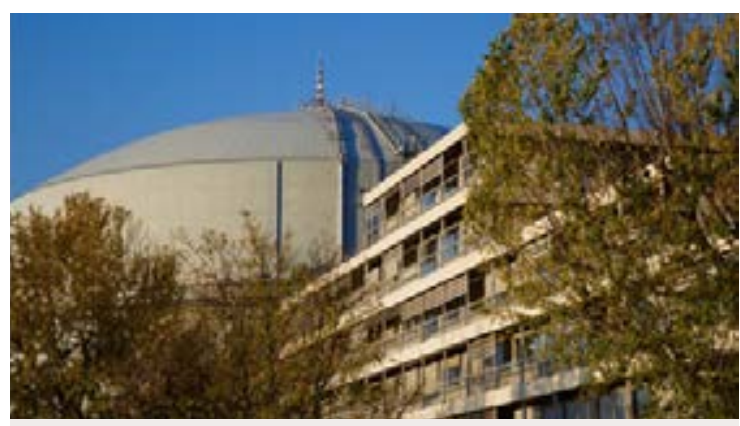

Type de l'infrastructure: TGIR

\section{Statut de l'infrastructure :}

Société civile de droit français

\section{Localisation de l'infrastructure:}

Grenoble

Représentantes de la France :

Amina TALEB-IBRAHIMI,

Patricia ROUSSEL-CHOMAZ

\section{Création: Exploitation:}

1969

1971

\section{Jouvence:}

1979-1987 : Second souffle (renouvellement infrastructures et instrumentation) 1991-1995 : Reconstruction complète à l'identique du cœur du réacteur 2001-2016 : Millennium (plan de renouvellement instrumental)

2002-2007 : Refit (mise en conformité de certaines infrastructures requise par l'ASN) 2012-2016 : Evaluations Complémentaires de Sûreté et renouvellement des éléments clés du réacteur

2015 : Endurance (plan de renouvellement instrumental)

Tutelles / Partenaires :

CNRS, CEA

\section{Contact en France :}

amina.taleb@cnrs-dir.fr

patricia.roussel-chomaz@cea.fr
L'ILL est une infrastructure européenne, avec 3 pays associés et 11 membres scientifiques, exploitant une source de neutrons à haut flux (58 MW). Ce réacteur alimente 38 instruments parmi les plus performants au monde dédiés à l'étude de la structure et de la dynamique de la matière. L'ILL met ses installations et son personnel à la disposition des scientifiques du monde entier (en moyenne, 1500 utilisateurs sont accueillis pour effectuer 800 expériences par an). Les recherches couvrent un large domaine scientifique : biologie, chimie, matière molle, physique fondamentale et nucléaire, science des matériaux. L'ILL reste aujourd'hui la source neutronique de référence au niveau mondial. Pour conserver cette place, I'ILL a démarré en 2001 un programme de modernisation, «Millennium», dont les 2 premières phases ont engendré un gain d'un facteur 24 sur le taux de détection. Une 3e phase de modernisation («Endurance» 2016-2026) débute, prévoyant 12 projets instrumentaux, la rénovation de 3 guides de neutron et l'amélioration du traitement des données et de l'environnement échantillon. La phase 1 (2016-2018) a été approuvée par le Comité de Direction en décembre 2015. L'ILL reste inégalé devant le plus récent réacteur européen de recherche (FRM-II en Allemagne), les nouvelles sources à spallation américaine et japonaise (SNS et J-PARC) et la source britannique ISIS

\section{IMPLICATIONS SOCIO-ÉCONOMIQUES}

Les installations sont utilisées par plus de 50 compagnies françaises et européennes pour des travaux de $R \& D$ et $30 \%$ des projets réalisés présentent un intérêt industriel [transports spatial, énergie, construction/ génie civil, médecine (production de radio-isotopes) et pharmaceutique].

Les retombées économiques pour la France et la région Rhône Alpes sont très importantes en termes d'emploi, de service et d'achats (par ex. $30 \mathrm{M€/an}$ de dépenses auprès des entreprises régionales). Le retour sur investissement est estimé à un facteur 3.5 .

\section{DONNÉES}

Estimation du flux de données : 100 à 200 To/an.

Stockage : Le stockage est assuré par l'ILL.

Accessibilité : L'ILL a une politique de l'accès aux données qui est la pionnière dans le domaine (programmes européens PaNdata).

\section{Coût de construction \\ $248 \mathrm{MF}(1971)$ \\ Refit $=29,7$ M€ (2007), Eléments \\ clés du réacteur $=27 \mathrm{M} €(2016)$, \\ Millennium (phases 1+2) = $77 \mathrm{M€}$ \\ Coût de fonctionnement : $94 \mathrm{M€}$ dont 24, 21 M€ de contribution \\ française. Plus pour la France \\ $8 \mathrm{M} €$ de TVA et 2,3 M€ de taxe \\ nucléaire.}

\section{Dimension internationale}

ILL, ESFRI Landmark

Coordinateur: William George Stirling

Pays partenaires : $F R, D E, U K$ à parts égales 


\section{ORPHEE/LLB}

\section{ORPHEE/Laboratoire Léon Brillouin}

Le réacteur Orphée est la source nationale de neutrons dédiée à la caractérisation de la matière. Avec une puissance de $14 \mathrm{MW}$, il est au 3e rang en Europe.

Le Laboratoire Léon Brillouin assure l'exploitation des faisceaux de neutrons sur des lignes d'expériences, principalement destinées à l'étude de la matière condensée, mises à la disposition de la communauté scientifique et industrielle.

Le LLB est à la fois un laboratoire de service, et un laboratoire de recherche en développant une activité scientifique propre et reconnue dans les domaines de la biologie/physicochimie, du magnétisme et la supraconductivité, des matériaux au sens large.

Ses missions sont d'une part la conception, la fabrication et le fonctionnement des 24 spectromètres performants installés à Orphée mais également d'assurer la coordination de la contribution française technique et scientifique à l'ESS (European Spallation Source). D'autre part, le LLB assure la formation (cours, TP, FAN...) de nouveaux utilisateurs de la diffusion neutronique et propose son expertise pour optimiser les expériences et l'exploitation des résultats.

L'utilisation de l'infrastructure Orphée/LLB représente $60 \%$ du temps total utilisé par la communauté française en diffusion de neutrons et génère un très fort taux de publications (3e rang mondial derrière ILL et ISIS).

\section{IMPLICATIONSSOCIO-ÉCONOMIQUES}

$15 \%$ du temps de faisceau est utilisé à des fins industrielles via des collaborations académiques ou contractuelles.

1 à 2 brevets/an sont déposés par les membres de l'infrastructure.

\section{DONNÉES}

Estimation du flux de données : $50 \mathrm{Go}$ et prochainement $100 \mathrm{Go} / \mathrm{an}$.

Stockage : Le stockage est assuré par un serveur de disque RAID de 25 To.

Accessibilité : Les données sont accessibles sur des serveurs internes.

\section{Coût de fonctionnement}

니 $B: 3,4 \mathrm{M} €$

Orphée : $8,5 \mathrm{M} €$

\section{Coût RH \\ Orphée : 60 ETPT LLB : 71 ETPT}

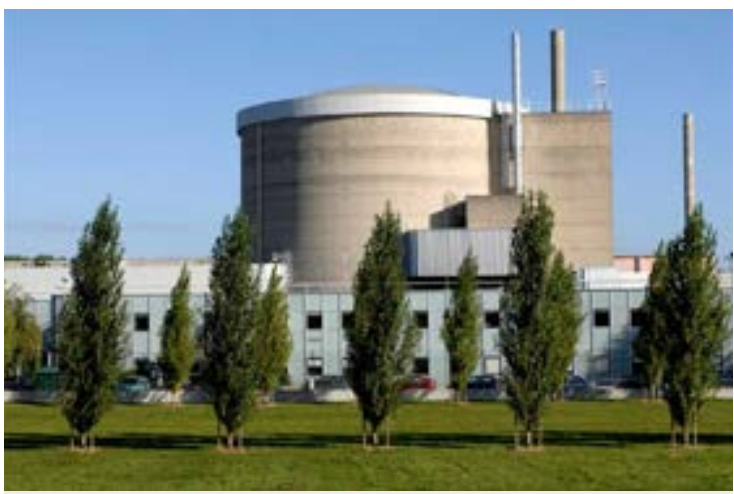

Type de l'infrastructure : TGIR

Statut de l'infrastructure:

Unité mixte de recherche

\section{Localisation :}

Gif-sur-Yvette

\section{Responsable de l'infrastructure :}

Christiane ALBA-SIMIONESCO

\begin{tabular}{|r|c|} 
Création : & Exploitation : \\
\hline 1974 & 1980 \\
\hline
\end{tabular}

\section{Tutelles / Partenaires :}

CEA, CNRS

\section{Contact en France :}

llb-sec@cea.fr

experience-llb@cea.fr

www-Ilb.cea.fr/index.php 


\section{Source Optimisée de Lumière d'Energie Intermédiaire du LURE}

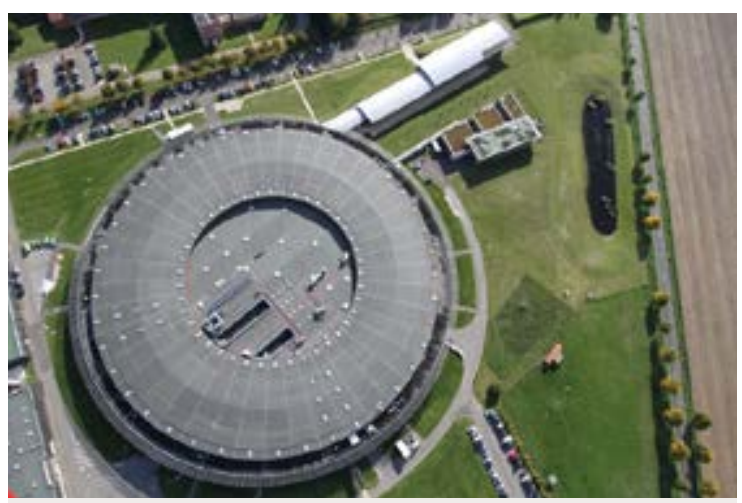

Type de l'infrastructure : TGIR

\section{Statut de l'infrastructure:}

Société Civile française

Localisation de l'infrastructure:

Gif-sur-Yvette

\section{Responsable de l'infrastructure :}

Jean DAILLANT

Création: Exploitation:

2002 2008

\section{Jouvence :}

2017 : 29 lignes

2020: Upgrade machine

\section{Tutelles / Partenaires :}

CNRS, CEA, Régions île-de-France et Centre,

Département de l' Essonne

\section{Contact en France :}

jean.daillan@synchrotron-soleil.fr

\section{www.synchrotron-soleil.fr}

SOLEIL est à la fois une source de lumière extrêmement brillante, une plateforme de service ouverte à toutes les communautés scientifiques et industrielles, et un laboratoire de recherche à la pointe des techniques expérimentales. Source nationale de rayonnement synchrotron, SOLEIL a pour but d'explorer la matière à différentes échelles.

Le rayonnement est produit par des électrons de très haute énergie $(2,75 \mathrm{GeV})$ circulant à une vitesse proche de celle de la lumière dans un anneau de $354 \mathrm{~m}$ de périmètre. Ce rayonnement s'étend de l'infrarouge aux rayons $X$, SOLEIL étant optimisé dans la gamme des rayons $X$ d'énergie intermédiaire. Le rayonnement produit est guidé vers les 29 lignes de lumière de SOLEIL, laboratoires instrumentés pour préparer et analyser les échantillons à étudier et traiter les informations recueillies.

Le spectre des méthodes d'analyse disponibles à SOLEIL couvre les spectroscopies (très haute résolution spectrale, résolues dans le temps), la diffraction et la diffusion (cinétique, cohérence), l'imagerie tridimensionnelle (très haute résolution spatiale, par contraste de phase, multi-échelle et multimodale).

SOLEIL, qui représente $10 \%$ des lignes européennes, est reconnu pour la qualité des faisceaux délivrés comme l'une des meilleures sources synchrotron au monde.

SOLEIL permet de mener des recherches fondamentales ou appliquées en physique, chimie, biologie, patrimoine, environnement ou sciences de l'univers.

\section{IMPLICATIONSSOCIO-ÉCONOMIQUES}

L'utilisation industrielle des lignes de SOLEIL (70 prestations en 2015 soit $230 \mathrm{k} €$, partenariats de R\&D et projets sélectionnés par les comités) représente plus de $10 \%$ du nombre de projets externes.

Depuis 2003, 26 brevets (11 inventions) déposés et 6 transferts de connaissances réalisés.

La construction et l'exploitation des équipements représente une forte retombée économique (en 2015, 3500 commandes pour $15 \mathrm{M€}$ ).

\section{DONNÉES}

Estimation du flux de données : plusieurs dizaines de To/jour de fonctionnement.

Stockage : Gestion autour du noyau TANGO. Plusieurs niveaux de stockage (local, primaire, secondaire ou long terme).

Accessibilité : Aux utilisateurs pendant 100 jours après la fin du projet.

Présence dans des réseaux de données : Collaboration informelle PaNDaaS entre installations neutrons et synchrotrons.

\section{Coût de construction \\ - \\ $473 \mathrm{M} €$ \\ Coût de fonctionnement : $63 \mathrm{M€}$}




\section{European X-ray Free Electron Laser}

XFEL est une source de Rayonnement-X très cohérent et sous forme d'impulsions de très courtes durées, en construction et dont l'exploitation est prévue en 2017. Sa brillance sera un milliard de fois plus élevée que celle des meilleures sources classiques de rayonnement $X$ et de très petites longueurs d'onde (0.05 nm) seront accessibles.

Le principe de fonctionnement de XFEL est basé sur un accélérateur linéaire supraconducteur d'une longueur de $2 \mathrm{~km}$ (dans un tunnel de $3,4 \mathrm{~km}$ ) qui confère une énergie de $17,5 \mathrm{GeV}$ à des paquets d'électrons (technologie développée à DESY en Allemagne). Ces électrons généreront, en traversant des onduleurs, des bouffées de rayons $X$ cohérents grâce au phénomène d'émission spontanée auto-amplifié (SASE). Ces flashs, de durée inférieure à 100 ms permettront des expériences inédites : la visualisation directe des mouvements atomiques et les analyses temporelles, l'imagerie de particules individuelles, la détermination de la structure de macromolécules... 6 stations expérimentales sont prévues dans un premier temps ouvrant de nouvelles perspectives dans de nombreux domaines.

Cette installation sera complémentaire de I'ESRF et de Soleil, qui sont basées en France. Au niveau européen SwissFEL en Suisse, infrastructure en construction à Villigen, aura des caractéristiques similaires et les deux installations dans le domaine spectrale XUV actuellement en opération : FERMI en Italie et FLASH en Allemagne, présentent une brillance extrêmement inférieure à celle de XFEL.

\section{DONNÉES}

Estimation du flux de données : Plusieurs Gigaflops sont attendus.

Stockage : La capacité de stockage sera de 10 Po, puis évoluera vers 100 Po.

Accessibilité : L'accès sera restreint aux utilisateurs internes et aux collaborateurs extérieurs proches.

\section{Coût de construction}

$1226 \mathrm{M} €(€ 2005)$

dont 38,5 M€

contribution française

Coût de fonctionnement : 2,4 M€ (FR)

\section{Dimension internationale \\ XFEL, ESFRI Landmark \\ Coordinateur : Massimo Altarelli, Directeur Général \\ Pays partenaires : FR (3,14\%),DE (57,8\%), RU (26,69\%), DK (0,96\%), ES $(0,96 \%)$, HU (0,96\%), IT (2,87\%), PL (2,16\%), SK (1,11\%), SE (1,52\%), $\mathrm{CH}(1,48 \%)$}

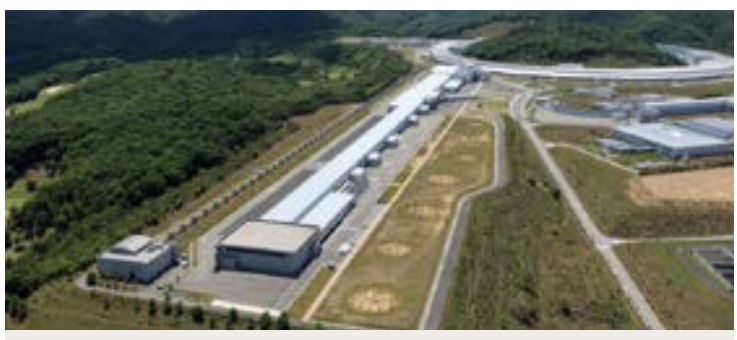

Type de l'infrastructure: TGIR

\section{Statut juridique de l'infrastructure :}

$\mathrm{GmbH}$

\section{Localisation :}

Hambourg, DE

\section{Représentantes de la France:}

Maria FAURY, Amina TALEB-IBRAHIMI

\section{Création :}

\section{Exploitation :}

2009

2017

\section{Tutelles / Partenaires :} CEA, CNRS

\section{Contact en France:}

maria.faury@cea.fr

amina.taleb@cnrs-dir.fr

\section{www.xfel.eu}




\section{Fédération des Accélérateurs pour les Études des Matériaux sous Irradiation}

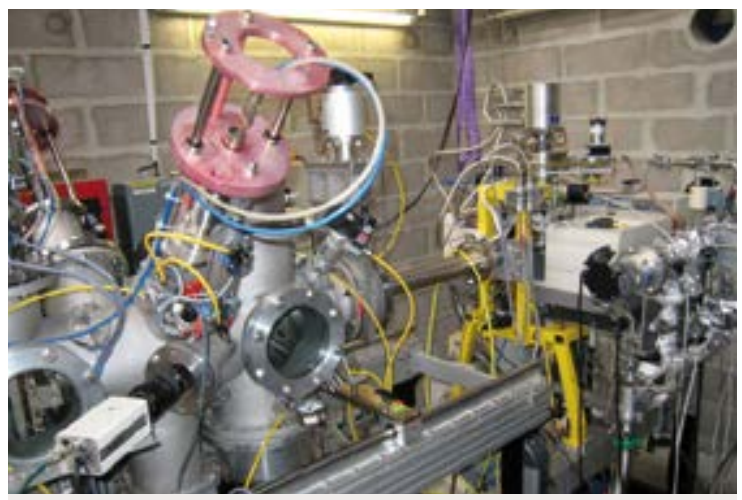

Type de l'infrastructure : IR

\section{Statut de l'infrastructure :}

Fédération de Recherche

Localisation de l'infrastructure:

Caen

\section{Localisation des autres sites :}

Orléans, Orsay, Palaiseau, Saclay

Responsable de l'infrastructure : Serge BOUFFARD

\section{Création :}

\section{Exploitation :}

2014

Tutelles / Partenaires:

CNRS, CEA, Ecole Polytechnique, Univ Orléans, Univ Caen-Normandie, Ensicaen

\section{Contact en France :}

bouffard@ganil.fr

\section{emir.in2p3.fr}

Le réseau national d'accélérateurs pour les études des matériaux sous irradiations (EMIR) offre aux chercheurs académiques et industriels de la communauté nationale et internationale l'accès à des moyens performants d'irradiation et de caractérisation. Environ 10 accélérateurs présentant une variété de particules (ions, électrons et neutrons) et d'énergie très étendue et répartis sur 5 sites (Caen/CIMAP, Orléans/CEMHTI, Orsay/CSNSM, Palaiseau/LSI, Saclay/SRMA, Saclay/SRMP) sont accessibles par appel à proposition.

Ces installations proposent différents types de caractérisation en ligne (Raman, RBS, DRX, Spectroscopie IR, MET...). EMIR assure la mise en réseau des installations, le suivi de leurs évolutions afin de mieux répondre aux besoins des expérimentateurs et des programmes de recherche, I'organisation des appels à proposition et enfin l'animation scientifique incluant la formation sur les effets des irradiations dans les matériaux.

Les domaines scientifiques les plus représentés concernent en premier chef la sécurité des installations nucléaires avec le vieillissement des matériaux de structure y compris le combustible et la gestion des déchets, mais également la microélectronique, les sciences de la terre, ainsi que la maitrise des défauts dans les études de physique des solides.

EMIR est la seule infrastructure au niveau national ou international à offrir ce panel d'irradiations.

\section{IMPLICATIONSSOCIO-ÉCONOMIQUES}

Une majorité des expériences sont en relation avec des préoccupations industrielles notamment celles de la Direction de l'Énergie Nucléaire du CEA, d'AREVA, d'EDF et des recherches sur les matériaux des futurs réacteurs de fusion.

\section{Coût de fonctionnement $0,83 \mathrm{M} €$}

Coût RH

8 ETPT 


\section{Réseau national de spectrométrie de masse FT-ICR à très haut champ}

Le réseau national de spectrométrie de masse par résonance cyclotronique des ions, à transformée de Fourier (FT-ICR) à très haut champ est une fédération qui regroupe les six laboratoires français spécialistes.

Elle a pour vocation de former une structure d'accueil délocalisée offrant à la communauté scientifique des équipements (30\% du temps disponible) de très haute résolution et précision ainsi que des compétences dans ce domaine.

Ses instruments (dotés de champ magnétique entre 7 et 9,4 T) sont maintenus au meilleur niveau et combinent des savoir-faire techniques et méthodologiques permettant de tirer le meilleur parti d'installations uniques en leur genre. Ses équipes de recherche couvrent les domaines de la santé et de la biologie, des matériaux du patrimoine, de la chimie analytique et de synthèse, de l'environnement.

La spectrométrie de masse FT-ICR est une technique permettant des performances ultra-élevées sur la mesure de masse et la capacité à discerner des ions de masses très proches. Elle apporte donc des informations essentielles pour l'identification d'espèces moléculaires par leurs formules chimiques exactes, y compris à des masses élevées et en mélange complexe. Couplée avec des sources d'ions variées et avec des techniques séparatives, elle permet d'analyser presque tout type d'échantillon, liquide, solide ou gazeux.

Certains sites sont communs avec d'autres infrastructures (IBiSa, MetaboHUB) et une synergie entre les IR RMN-THC, RENARD (RPE) et le réseau FT-ICR permet de couvrir un ensemble de besoins analytiques.

\section{IMPLICATIONSSOCIO-ÉCONOMIQUES}

Certains sites sont particulièrement impliqués dans des projets régionaux d'importance comme Metz dans le projet Verbilor (Valorisation énergétique en réacteur haute température de la biomasse en Lorraine) ou Lille dans I'Institut de transition énergétique (ITE) IFMAS (Institut français des matériaux agrosourcés).

\section{DONNÉES}

Estimation du flux de données : Max 1 To/jour

Stockage : Chaque site gère le stockage des données

$$
\begin{aligned}
& \text { Coût de construction } \\
& \text { 5,8 M€ } \\
& \text { Coût de fonctionnement: 0,349 M€ }
\end{aligned}
$$

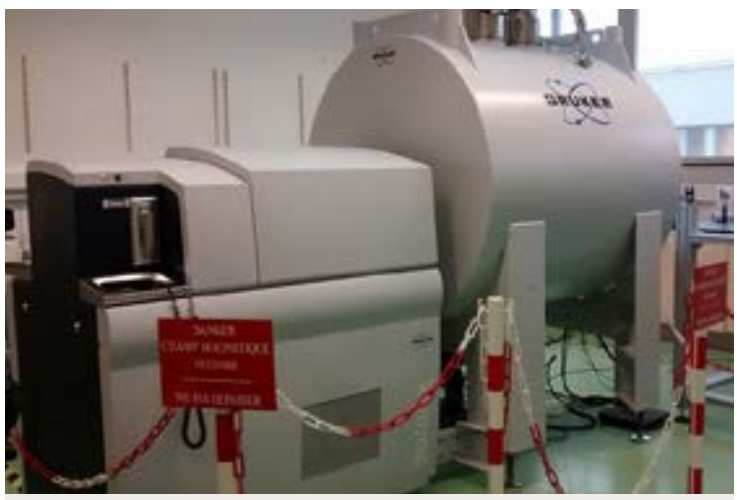

Type de l'infrastructure : IR

Statut de l'infrastructure :

Fédération de Recherche CNRS

\section{Localisation :}

Palaiseau

Localisation des autres sites :

Paris, Lille, Metz, Orsay

Responsable de l'infrastructure: Guillaume VAN DER REST

\begin{tabular}{|r|c|}
\hline Création : & Exploitation : \\
\hline 2010 & 2010 \\
\hline
\end{tabular}

\section{Tutelles / Partenaires :}

CNRS, École polytechnique, ESPCI ParisTech, Univ. Lille 1, Univ. Lorraine, Univ. Pierre et Marie Curie, Univ. Paris Sud

\section{Contact en France :}

guillaume.van-der-rest@u-psud.fr 


\section{des Champs Magnétiques Intenses}

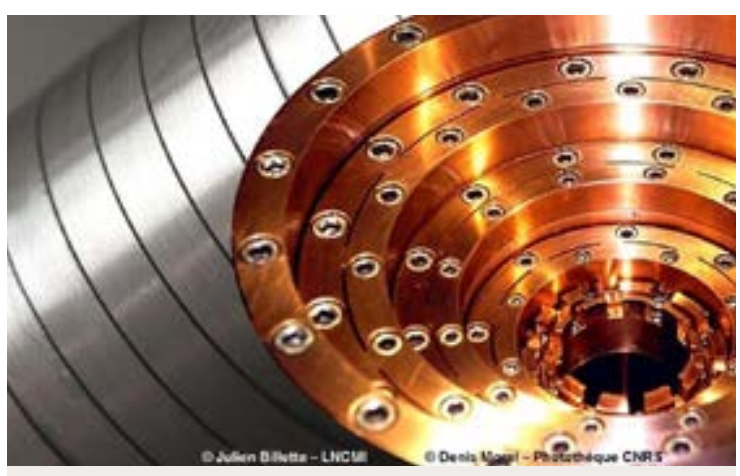

Type de I'infrastructure : IR

Statut de l'infrastructure :

Unité propre de recherche

\section{Localisation de l'infrastructure:}

Toulouse

\section{Localisation des autres sites :}

Grenoble

Responsable de l'infrastructure :

Geert RIKKEN

\section{Exploitation :}

2009

Tutelles / Partenaires :

CNRS, INSA Toulouse, Univ. Toulouse, UGA

\section{Contact en France:}

Incmi.direction@Incmi.cnrs.fr

\section{Incmi.cnrs.fr}

Le Laboratoire National des Champs Magnétiques Intenses (LNCMI) est une infrastructure de recherche qui accueille des scientifiques du monde entier pour des expériences en champ intense.

Le LNCMI est la plus grande infrastructure européenne de recherche sous champs intenses et deuxième au niveau mondial, derrière le NHMFL (USA). Sur le site de Grenoble, le LNCMI offre des champs magnétiques statiques allant jusqu'à 36T, sur le site de Toulouse, des champs magnétiques pulsés allant jusqu'à 91 T de manière non-destructive et 180 T de manière semi-destructive.

Le LNCMI assure le développement des installations électrotechnique et hydraulique, des aimants ainsi que l'instrumentation scientifique pour les mesures physiques sous champs magnétiques intenses. II donne l'accès aux installations des champs intenses à des utilisateurs via des appels à projet, et soutient l'implémentation, l'interprétation et la valorisation des expériences.

De nombreuses expériences de mesures physiques sous champs intenses sont disponibles (spectroscopie UV-VIS-NIR-THz, RMN, RPE, aimantation, transport...).

Les communautés utilisatrices principales sont celles des supraconducteurs, de la physique des semi-conducteurs et des nanostructures, et du magnétisme.

\section{IMPLICATIONS SOCIO-ÉCONOMIQUES}

En moyenne, 2 contrats de recherche industriels sont en cours pour développer des sources de champs magnétiques ou pour tester des matériaux ou dispositifs sous champs intenses ou sous lévitation magnétique.

\section{DONNÉES}

Estimation du flux de données : $1 \mathrm{Go} / a n$

$$
\begin{aligned}
& \text { Coût de fonctionnement } \\
& \text { 3,5 M€ }
\end{aligned}
$$

\section{Coût RH}

\section{Dimension internationale \\ AISBL 'European Magnetic Field Laboratory' (EMFL) \\ Coordinateur : Martin van Breukelen (NL) \\ Pays partenaires : FR, DE, NL, UK \\ Site internet : www.emfl.eu/home.html}




\section{Laboratoire d'Utilisation des Lasers Intenses}

L'infrastructure de recherche LULI est le pôle civil français des lasers de puissance de forte énergie.

II met à disposition du plus grand nombre d'utilisateurs, nationaux et internationaux, des chaînes laser compétitives, au plus haut niveau international, et des espaces expérimentaux dotés d'une instrumentation de pointe, adaptés aux recherches sur la physique des plasmas créés par laser. II exploite I'installation LULI2000, sur le site de l'Ecole Polytechnique, construit et exploitera l'installation APOLLON, sur le site de l'Orme des Merisiers, et conduit des développements spécifiques dans le but d'améliorer continûment leurs performances et de répondre à la demande des utilisateurs.

LULI2000, grâce au couplage sur cible d'impulsions de forte énergie et de forte puissance, permet de reproduire en laboratoire et d'étudier des plasmas lointains (intérieurs planétaires, étoiles...), possiblement magnétisés, et de comprendre la physique de certains concepts de production d'énergie par fusion.

APOLLON, qui devrait à terme atteindre une puissance de $10 \mathrm{PW}$, produira, grâce à son intensité lumineuse extrême, des faisceaux de particules et des rayonnements aux paramètres inégalés, permettant de repousser les limites de la recherche fondamentale.

Ouvert à la communauté scientifique nationale et internationale en 2018, il permettra d'explorer des sujets novateurs (physique en champ fort, accélération de particules au-delà du GeV) et des applications pluridisciplinaires innovantes.

\section{IMPLICATIONSSOCIO-ÉCONOMIQUES}

L'infrastructure est un acteur important dans les activités de R\&D du Plateau de Saclay. Douze brevets ont été déposés depuis 2004 (notamment dans le domaine des lasers solides pompés par diode) et 2 start-ups ont été cofondées. Des activités de transfert technologique, notamment vers Thales, ont de plus démarré dans le cadre du projet APOLLON.

\section{Coût de construction}

8 M€LULI2000 + 27 M€ APOLLON (hors personnel)

Coût de fonctionnement

de 3,7 M€ (2015) à 4,9 M€ (2020)

(hors personnel)

\section{Dimension internationale \\ LASERLAB-EUROPE}

Coordinateur : LLC (SE)

Pays partenaires : 30 laboratoires sur 16 pays; 20 infrastructures

Site internet : www.laserlab-europe.net

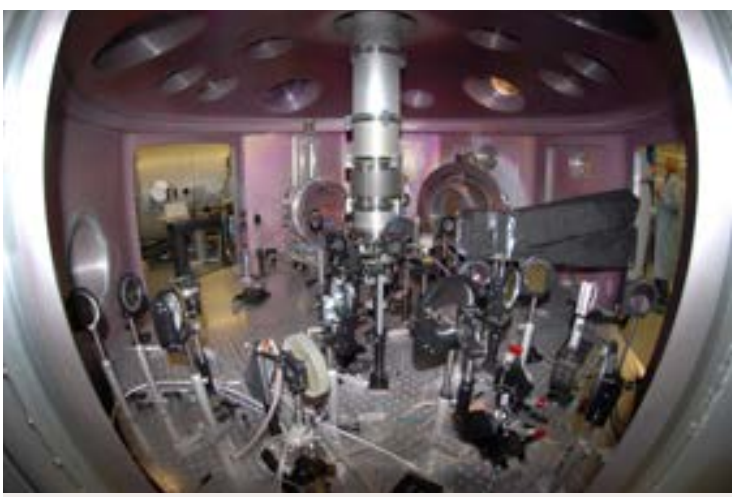

Type de l'infrastructure : IR

Statut de l'infrastructure :

Unité mixte de recherche

\section{Localisation :}

Palaiseau

Localisation des autres sites : Gif-sur-Yvette

\section{Responsable de l'infrastructure:} Patrick AUDEBERT

\section{Création: Exploitation: \\ 1994 \\ 2003 (LULI) et 2018 (Apollon)}

\section{Tutelles / Partenaires :}

CNRS, Ecole Polytechnique, CEA, UPMC

\section{Contact en France:}

patrick.audebert@polytechnique.edu luli@luli.polytechnique.fr 


\section{Microscopie Electronique en Transmission et Sonde Atomique}

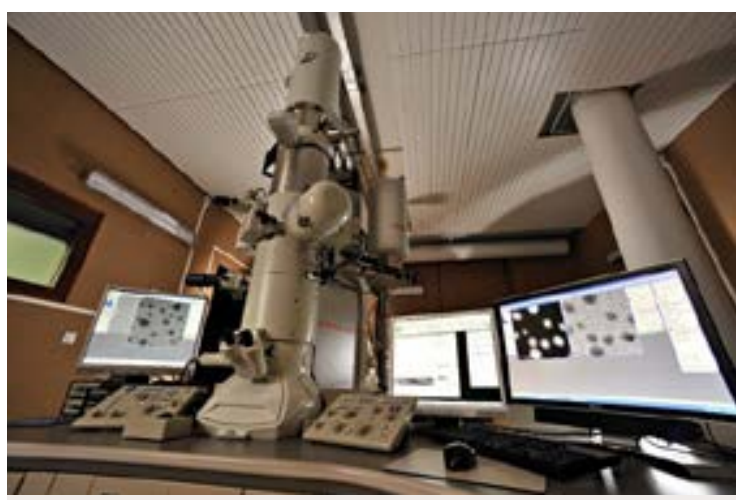

Type de l'infrastructure : IR

Statut de l'infrastructure :

Fédération de Recherche CNRS

Localisation de l'infrastructure :

Orsay

Localisation des autres sites :

Strasbourg, Toulouse, Lyon, Rouen, Caen,

Paris, Marseille, Grenoble

Responsable de l'infrastructure :

Mathieu KOCIAK

Création: Exploitation:

2009

2012

Tutelles / Partenaires :

CNRS, CEA, Universités

\section{Contact en France :}

mathieu.kociak@u-psud.fr

\section{www.metsa.fr}

METSA est un réseau national de 8 plates-formes régionales qui met à la disposition de la communauté scientifique des instruments uniques en France dans le domaine de la Microscopie Electronique en Transmission et de la Sonde Atomique. Les 8 plates-formes sont: IRMA : GPM, Rouen et CRISMAT, Caen; CEMES, Toulouse; IM2NP et CINAM, Marseille; CLYM, Lyon; PFNC-Minatec-CEA, Grenoble; IPCMS, Strasbourg; MPQ, Paris; LPS, Orsay. Ce réseau comprend en 201613 MET (à émission de champ, corrigés, analytiques/spectroscopiques, in situ, holographiques, 3D), 6 FIB/SEM associés et 3 Sondes Atomiques (tomographiques et analytiques).

Les missions de la fédération sont l'accueil des scientifiques au travers de 2 appels à projets annuels, la recherche en développements instrumentaux, méthodologiques et en modélisation afin de garantir un parc expérimental de haut niveau, la formation (par sa participation à des stages ou workshops), et le conseil pour l'implantation de nouveaux équipements MET et SA en vue de favoriser tout effort de mutualisation, de développement et de complémentarité à partir de l'environnement existant.

Les thèmes scientifiques les plus représentés sont associés aux compétences des plateformes et concernent la Physique des Matériaux au sens large : nanotechnologies/nanosciences, chimie des matériaux, ingénierie des matériaux et matériaux pour la santé.

\section{IMPLICATIONSSOCIO-ÉCONOMIQUES}

La majeure partie des expériences accueillies par les plateformes de l'infrastructure repose sur des projets de recherche et d'innovation (thèses, ANR, R\&D industrielle et relations avec industriels, pôles de compétitivité).

\section{DONNÉES}

Estimation du flux de données : qq dizaines de To/an par an réparties sur les 8 plateformes.

Stockage : Au sein des plateformes

Accessibilité : Accessibles aux porteurs du projet

\section{Coût de fonctionnement - \\ $0,25 \mathrm{M€}$}

\section{Dimension internationale}

2 plateformes de l'IR sont associées au réseau européen ESTEEM2

Coordinateur : Etienne SNOECK (FR, Toulouse)

Pays partenaires : $\mathrm{FR}, \mathrm{SE}, \mathrm{UK}, \mathrm{BE}, \mathrm{NL}, \mathrm{PL}, \mathrm{SI}, \mathrm{DE}, \mathrm{ES}, \mathrm{AT}$

Site internet : esteem2.eu

\section{Coût RH}

4 ETPT 


\section{PETAwatt Aquitaine Laser}

PETAL est un laser de haute énergie et de haute puissance, au sein de l'installation Laser Mégajoule (LMJ). PETAL génère une impulsion de l'ordre du kJ et d'une durée de 0,5 à 10 picosecondes couplée avec les faisceaux de haute énergie du LMJ.

Les objectifs de PETAL sont:

1. L'obtention en laboratoire d'états de la matière en conditions extrêmes, représentatifs des cœurs des planètes ou des étoiles. Ces conditions extrêmes sont obtenues par compression en utilisant les faisceaux laser du LMJ, et par chauffage isochore (dépôt rapide d'énergie) à l'aide de PETAL.

2. L'étude de la fusion par confinement inertiel (FCI), en particulier les phénomènes physiques clefs du schéma d'«allumage rapide», et l'étude du schéma d' "allumage par choc », en créant des rayonnements brefs pour radiographier les cibles comprimées par le LMJ.

3. L'étude de phénomènes astrophysiques simulés en laboratoire, telles les instabilités hydrodynamiques lors d'explosions de supernovæ, la génération de champs magnétiques intenses et de jets astrophysiques, l'« opacité » de la matière. Afin d'accroître les connaissances sur l'Univers.

4. La physique des particules et la physique nucléaire, avec la génération de faisceaux de protons énergétiques, pour sonder les plasmas, ou pour l'étude de la protonthérapie (traitement des tumeurs cancéreuses), les réactions nucléaires dans les plasmas, la nucléosynthèse, et l'activation des noyaux.

Les objectifs sont détaillés dans «LMJ-PETAL Scientific Case» (www-Imj. cea.fr/en/ForUsers.htm).

\section{IMPLICATIONSSOCIO-ÉCONOMIQUES}

Le développement des technologies nécessaires a donné lieu à des collaborations scientifiques du CEA avec des laboratoires nationaux et internationaux. Les principales innovations ont porté sur les composants optiques et leur traitement, le pilote laser et le compresseur. Le CEA s'est appuyé sur de nombreux industriels, qui sont pour la plupart membres du Pôle de Compétitivité Route des Lasers.

\section{DONNÉES}

Estimation du flux de données : $10 \mathrm{Go} / a n$ et $50 \mathrm{Go} / a n$ à moyen terme.

Stockage : Sur moyens informatiques sécurisés du CEA et de l'Université de Bordeaux

\section{Coût de construction \\ 54,3 M€ (2007), 15,6 M€ \\ (intégration dans le LMJ) \\ PIA Equipex «PETAL+» pour la réalisation des 1 ers diagnostics, pour un montant de $8 \mathrm{M€}$ Coût de fonctionnement : 1,3 M€}

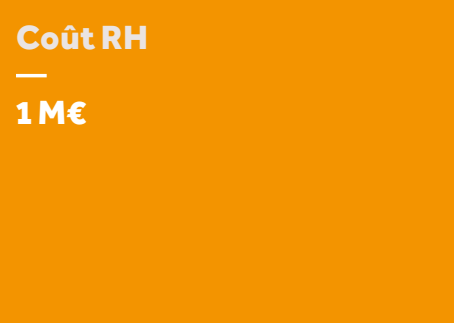

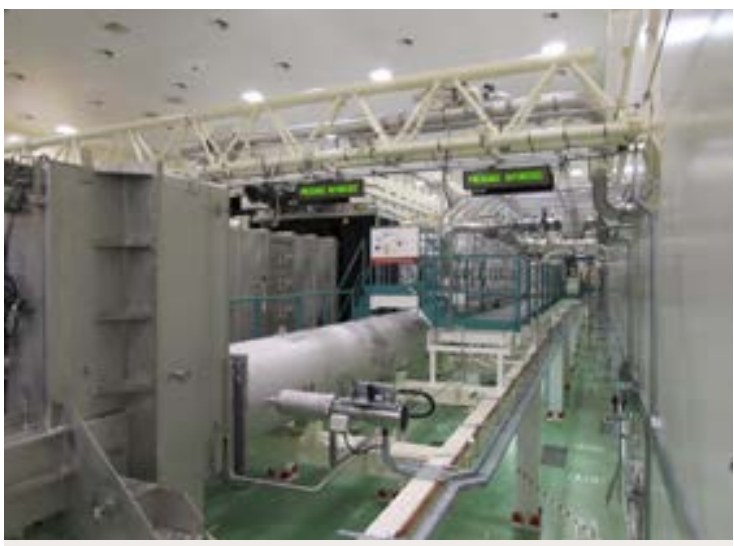

Type de l'infrastructure : IR

\section{Localisation :}

Le Barp

Responsable de l'infrastructure: Jean-Pierre GIANNINI

\section{Création :}

Exploitation:

2008

\section{Tutelles / Partenaires : \\ CEA, Région Aquitaine}

\section{Contact en France :} userLMJ@cea.fr

\section{petal.aquitaine.fr}




\section{Résonance Magnétique Nucléaire à Très Hauts Champs}

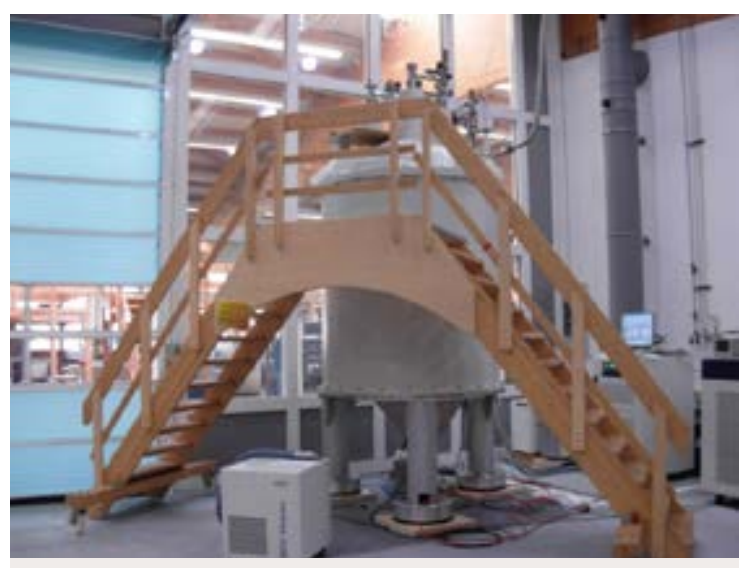

Type de l'infrastructure : IR

\section{Statut de l'infrastructure :}

Fédération de Recherche

Localisation de l'infrastructure :

Grenoble

\section{Localisation des autres sites :}

Paris, Bordeaux, Orléans, Lille, Lyon,

Gif-sur-Yvette

Responsable de l'infrastructure: Jean-Pierre SIMORRE

\section{Création :}

\section{Exploitation :}

2007 2008

\section{Jouvence :}

2016 Nouveau spectromètre $1,2 \mathrm{GHz}$

\section{Tutelles / Partenaires :}

CNRS, CEA, UGA, Univ. Lille, UCBL, ENSL, UBx1, IPB, UBxS, INSERM, UPMC, ENS Paris

\section{Contact en France :}

jean-pierre.simorre@ibs.fr

\section{www.ir-rmn.fr}

L'infrastructure RMN-THC est constituée d'équipes de recherche en RMN qui opèrent et encadrent l'utilisation des spectromètres RMN à très hauts champs français.

Ce réseau est une structure d'accueil ouverte à la communauté scientifique nationale et internationale. Il est décentralisé sur 7 laboratoires de recherche et compte 11 spectromètres de RMN à THC de $750 \mathrm{Mhz}$ à $1 \mathrm{GHz}$. Les possibilités d'accès à des équipements de pointe sont accompagnées d'une expertise et d'un support scientifique reconnu au niveau international.

Pluridisciplinaire, le réseau couvre un large domaine d'applications en biologie, chimie, physique, sciences de l'Univers, médecine... $30 \%$ du temps disponible sur ses spectromètres RMN est distribué par l'intermédiaire d'appels à projets. L'infrastructure permet ainsi de fédérer, de dynamiser et de valoriser la recherche ainsi que de coordonner et d'optimiser les investissements nationaux dans le domaine de la RMN à très hauts champs.

Au niveau international, la France est extrêmement bien placée en détenant deux spectromètres $950 \mathrm{MHz}$ sur les cinq existants, un spectromètre $1 \mathrm{GHz}$, le plus puissant au monde, ainsi que des équipements uniques (cryosondes, DNP et sondes de RMN solide). La position de leadership de la France sera renforcée par l'acquisition d'un spectromètre $1,2 \mathrm{GHz}$ qui sera installé à Lille en fin de décennie.

\section{IMPLICATIONSSOCIO-ÉCONOMIQUES}

L'utilisation de l'IR RMN-HTC contribue aux avancées scientifiques dans les axes de recherche sociétaux (santé, environnement, information, nanotechnologies...) en partenariat avec des pôles de compétitivité, (Elastopôle, Cosmetic Valley, MAUD, NSL, BIOASTER...), des instituts Carnot et des industriels (Cephalon, Alcan, Michelin, Messier-Bugatti...).

Plusieurs brevets sont déposés par an et par site.

\section{Coût de construction \\ Projet 1,2GHz: $15 \mathrm{M€}$ \\ Coût de fonctionnement : 1,2 M€}

\section{Coût RH}




\section{REseau NAtional de Résonance paramagnétique électronique interDisciplinaire}

La fédération Renard est une infrastructure décentralisée regroupant 27 spectromètres RPE (dont 3 RPE onde continue équipés en ENDOR, 6 RPE impulsionnels et 4 RPE équipés en imagerie, dont 1 à gradients pulsés) à la pointe de la technologie moderne, répartis sur 10 laboratoires et 5 villes.

Outre la mise à disposition à la communauté scientifique de ces instruments ainsi que d'une expertise reconnue en RPE, par le biais d'appels à projet, Renard a pour mission de soutenir les plates-formes existantes ou à venir en termes de fonctionnement et de personnel et d'optimiser l'accessibilité à une communauté scientifique élargie. Le rôle de la fédération est également d'organiser et de programmer le développement de ces plates-formes en assurant l'implantation en France des appareillages les plus avancés là où sont les compétences scientifiques.

Ces plateformes constituent des lieux d'échanges interdisciplinaires en chimie, physique, biologie, sciences de la Terre au plus haut niveau scientifique, où sont mutualisées des techniques complémentaires et les compétences apportées par des thématiques scientifiques différentes.

\section{IMPLICATIONSSOCIO-ÉCONOMIQUES}

Les industriels utilisateurs actuels de la Fédération sont les sociétés TOTAL, HUTCHINSON, SANOFI, I'OREAL.

De très fortes implications sont aussi à remarquer avec le CNES pour la spatialisation de la RPE et l'IRSN pour la dosimétrie prédictive.

\section{DONNÉES}

Estimation du flux de données : 1 To pour l'ensemble de la fédération. Stockage : organisé au niveau de chaque site.

Accessibilité : Données accessibles.

$$
\begin{aligned}
& \text { Coût de fonctionnement } \\
& \text { 0,3 M€ }
\end{aligned}
$$

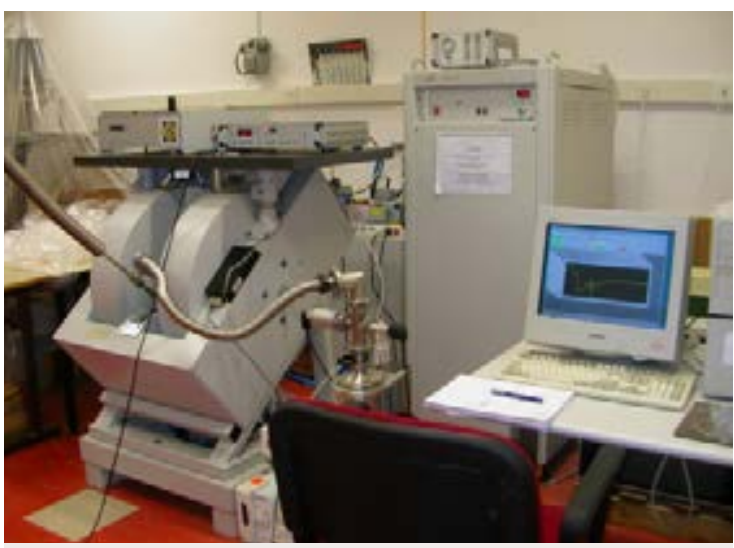

Type de l'infrastructure : IR

Statut juridique de l'infrastructure:

Fédération de Recherche

\section{Localisation :}

Lille

\section{Localisation des autres sites :}

Strasbourg, Grenoble, Marseille, Paris

\section{Responsable de l'infrastructure :}

Hervé VEZIN

\begin{tabular}{|r|c|} 
Création : & Exploitation : \\
\hline 2012 & 2012 \\
\hline
\end{tabular}

\section{Jouvence :}

2015-2017 ( 2 nouveaux spectromètres)

\section{Tutelles / Partenaires :}

CNRS, AMU, UTLN, UMPC, Chimie ParisTech, Univ. Paris Descartes Paris V, Univ. Lille, Unistra, UGA, CEA

\section{Contact en France :}

herve.vezin@univ-lille1.fr 


\section{Réseau NAtional des grandes centrales de TECHnologies}

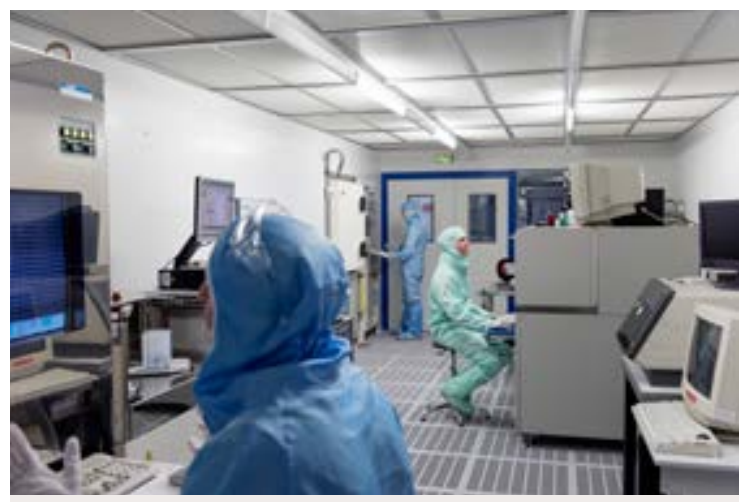

Type de l'infrastructure : IR

Statut de l'infrastructure :

Groupement d'Intérêt Scientifique

Localisation de l'infrastructure:

Orsay

Localisation des autres sites :

Lille, Besançon, Marcoussis, Grenoble,

Toulouse

Responsable de l'infrastructure:

Michel DE LABACHELERIE

Création: Exploitation: Jouvence:

2003

2003

2016-2020

Tutelles / Partenaires :

CNRS, Universités

\section{Contact en France :}

michel.labachelerie@cnrs-dir.fr

\section{www.renatech.org}

Le réseau Renatech regroupe des infrastructures et des moyens lourds en micro et nanotechnologie répartis sur le territoire national au sein de 6 centrales fortement impliquées dans le développement technologique, rassemblant des compétences scientifiques allant du matériau au système. Ces laboratoires représentent $7000 \mathrm{~m}^{2}$ de salles blanches dotées d'équipements de pointe ouverts à la communauté scientifique. Les domaines scientifiques adressés sont la microélectronique, la photonique, les micro-systèmes électromécaniques (MEMS), les micro- et nanotechnologies pour la biologie et la caractérisation et l'instrumentation.

Les grandes centrales technologiques sont:

- I'Institut d'Electronique, de Microélectronique et de Nanotechnologie (IEMN) à Lille;

- I'Institut d'Electronique Fondamentale (IEF) à Orsay;

- le Laboratoire de Photonique et de Nanostructures (LPN) à Marcoussis:

- I'InstitutFEMTO-ST (Franche-Comté, Electronique Mécanique Thermique et Optique-Sciences et Technologies) à Besançon;

- le laboratoire des technologies de la microélectronique (LTM) à Grenoble et le laboratoire d'Analyse et d'Architecture des Systèmes (LAAS) à Toulouse.

Ce réseau permet à l'ensemble des laboratoires de la communauté nationale de bénéficier d'un accès à ces moyens qui permettent de concevoir, de fabriquer des micro-nanoobjets, des micro-nanosystèmes et de les intégrer. L'apport de l'IR s'étend du fondamental aux filières technologiques.

\section{IMPLICATIONSSOCIO-ÉCONOMIQUES}

Renatech est un outil technologique sur lequel s'appuie une large communauté académique et industrielle. Chaque année, un service de micro- et nanofabrication est fourni à une centaine d'entreprises, dont la moitié sont des PME. Les activités de Renatech ont donné lieu à 29 brevets prioritaires en 2013. Le réseau possède un portefeuille de 147 brevets. Par ailleurs, 10 startups ont été créées depuis 2009.

\section{Coût de construction \\ $200 \mathrm{M} €$ \\ Coût de fonctionnement : $8 \mathrm{M€}$}

\section{Coût RH \\ 140 ETPT}


SCIENCES DE LA MATIÈRE ET INGÉNIERIE 

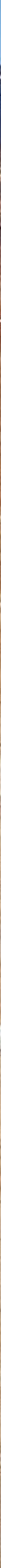


\section{Astronomie et Astrophysique}

L'astrophysique s'intéresse à l'histoire de l'univers et de ses constituants, depuis ses débuts dominés par des phénomènes physiques encore mal appréhendés à sa complexification croissante, avec la formation et l'évolution des galaxies, des étoiles et des systèmes planétaires, jusqu'à l'apparition de la vie. Elle repose sur des observations toujours plus détaillées, s'appuie sur des travaux théoriques et des modélisations numériques et s'ouvre aux expériences de laboratoire. Le niveau de précision nécessaire pour contraindre les modèles nécessite des observatoires au sol et dans l'espace toujours plus performants qui rentrent désormais, en raison de leur complexité, de leur coût et de leur internationalisation, dans la classe des TGIR. Au-delà de l'observation classique dans le domaine optique, l'observation s'est élargie à tout le spectre électromagnétique et s'ouvre désormais à de nouveaux messagers comme les ondes gravitationnelles ou les neutrinos. Cette analyse multispectrale et multi-messagers permet de couvrir les différents processus physiques impliqués, de parcourir l'histoire de l'univers et de ses constituants. La mise en œuvre de ces moyens d'observations, les moyens d'analyse et de stockage des données, ainsi que les simulations numériques, se font pour leur grande majorité dans un cadre international, bilatéral pour les infrastructures de taille moyenne, dans un cadre européen pour la plupart, et à l'échelle mondiale dans quelques cas.

La stratégie de la discipline en matière d'infrastructures de recherche s'appuie sur la réflexion menée lors des exercices de prospective scientifique organisés tous les cinq ans par le CNRS-INSU, qui élaborent les priorités d'évolution des moyens avec l'ensemble des acteurs institutionnels et la communauté scientifique; elle prend en compte les stratégies européennes et internationales au sol et dans l'espace, les interfaces avec d'autres disciplines et les sorties des séminaires de prospective scientifique organisés régulièrement par le CNES pour le volet spatial. Les conclusions des dernières prospectives menées par I'INSU et le CNES en 2014 servent naturellement d'entrée pour la feuille de route nationale. Depuis l'exercice de 2009, la prospective nationale s'articule avec les stratégies européennes définies par l'ERA-NET ASTRONET, qui regroupe les agences de financement de l'astronomie et couvre tous les aspects de la discipline, de l'étude du Soleil et du système solaire à celle des confins de l'univers, et par l'ERA-NET ASPERA, dédié aux astroparticules, et son successeur le Consortium APPEC. La feuille de route européenne ESFRI et le programme Cosmic Vision de
I'ESA jouent également un rôle structurant. Les questions scientifiques clés identifiées dans la «Vision scientifique pour l'astronomie européenne n produite par ASTRONET en 2007 et mise à jour en 2013, endossées par la prospective nationale, sont les suivantes: Comprenons-nous le fonctionnement de l'univers dans les conditions extrêmes que l'on y rencontre? Comment les galaxies, les étoiles, les planètes se forment-elles et évoluent-elles? Comment notre système solaire et nous-mêmes trouvons-nous notre place?

\section{L'Organisation Internationale ESO (European Southern} Observatory) opère sur ses sites du Chili de très grands télescopes au sol. Elle gère aujourd'hui les observatoires optiques de La Silla et du Paranal (LSP, qui inclut le VLT/ VLT-I), ainsi que la participation européenne à l'observatoire radio millimétrique et submillimétrique ALMA. Aces télescopes s'ajoutera l'observatoire optique E-ELT (European Extremely Large Telescope), labellisé «landmark » dans le paysage ESFRI, dont la construction a démarré. Au-delà de ces trois composantes internationales, l'ESO se décline aussi à travers une IR nationale INSTRUM-ESO (Instrumentation pour les grands télescopes de l'ESO). Ces développements instrumentaux coordonnés au plan national sont indispensables pour assurer la place de la France dans le projet en termes de retour industriel national. Par ailleurs, ils positionnent la France au meilleur niveau scientifique dans le cadre européen grâce à l'expertise sur les instruments et leurs données.

Plus spécialisées et plus accessibles, deux TGIR en partenariat international, le CFHT à Hawaï dans le domaine de I'optique (CA, FR, US) et I'IRAM près de Grenoble dans le domaine milli- et submillimétrique (DE, $F R, E S)$, offrent des performances uniques en imagerie grand champ, spectropolarimétrie et sondage de l'univers froid et complètent les télescopes de l'ESO et les missions spatiales.

Deux IR multilatérales, le radiotélescope en réseau ILT (International Lofar Telescope) et le télescope Cherenkov HESS (High Energy Spectroscopic System) sont les précurseurs de deux grands projets internationaux inscrits sur la feuille de route ESFRI : SKA et CTA. CTA est aujourd'hui sur la feuille de route nationale, en projet de TGIR. Il est à noter que les infrastructures HESS aujourd'hui et CTA dans le futur, essentiellement dédiées à l'astrophysique des hautes énergies, se situent à l'interface de l'astrophysique et de la physique des particules. 
Cet ensemble observationnel est complété d'une IR virtuelle, le Centre de données astronomiques de Strasbourg (CDS) pour la diffusion des données astronomiques et de produits à valeur ajoutée.

En complément, la mise en réseau des plateformes instrumentales pour les grands projets "sol " et "espace " permet d'optimiser leur rôle dans le développement de ces projets et dans la mise en œuvre de moyens de haute technologie, en liaison forte avec le monde industriel et le tissu local.

II faut également citer trois moyens internationaux plus ciblés : JIV-ERIC pour le VLBI et les systèmes de référence spatio-temporels (physique fondamentale), les radars ionosphériques (EISCAT/EISCAT3D), et le télescope solaire THEMIS associé au projet du futur EST (European Solar Telescope) et trois moyens nationaux : le Télescope du Pic du Midi (spectropolarimétrie stellaire), le Télescope de l'Observatoire de Haute Provence (recherche d'exoplanètes) et le Radio Télescope de Nançay (pulsars).

La communauté est aussi utilisatrice d'autres infrastructures de recherche, notamment les moyens de calcul et de stockage nationaux et européens pour les développements théoriques, les simulations numériques et le traitement massif de données et les lignes de lumière pour l'astrophysique de laboratoire; on doit aussi citer l'expérience VIRGO (ondes gravitationnelles et lois physiques fondamentales) et le LSST (ouverture du domaine de l'univers temporellement dynamique, matière et énergie noires), portés par la physique des particules. Astrophysique et physique des particules tendent à se rapprocher autour des questions sur l'unification des interactions fondamentales et le rôle particulier de la gravitation, les astroparticules et les très hautes énergies. Le domaine des neutrinos d'ultra haute énergie (KM3NeT, Ice-Cube) est un autre exemple de ce champ interdisciplinaire.

\section{LISTE DES INFRASTRUCTURES DE RECHERCHE} DANS LE DOMAINE ASTRONOMIE ET ASTROPHYSIQUE

\begin{tabular}{|c|c|c|c|}
\hline CATÉGORIE & NOM & NOM COMPLET & ESFRI \\
\hline \multirow[t]{4}{*}{ OI } & ESO & European Southern Observatory & \\
\hline & ESO ALMA & Atacama Large Millimeter/Submillimiter Array & \\
\hline & ESO EELT & European Extremely Large Telescope & E-ELT (2006) \\
\hline & ESO LSP & La Silla \& Paranal Observatory & \\
\hline TGIR & CFHT & Canada-France-Hawaii Telescope & \\
\hline TGIR & IRAM & Institut de RadioAstronomie Millimétrique & \\
\hline IR & CDS & Centre de Données astronomiques de Strasbourg & \\
\hline IR & ESO-INSTRUM & $\begin{array}{l}\text { Instrumentation pour les grands télescopes } \\
\text { de l'ESO }\end{array}$ & \\
\hline IR & HESS $^{1}$ & High Energy Stereoscopic System & \\
\hline IR & ILT-LOFAR FR & International Low Frequency Radio Array Telescope & \\
\hline Projet & $C T A^{2}$ & Cherenkov Telescope Array & CTA (2008) \\
\hline
\end{tabular}

1 Relève également du secteur «Physique nucléaire et des hautes énergies ".

2 Relève également du secteur «Physique nucléaire et des hautes énergies ». 


\section{European Southern Observatory}

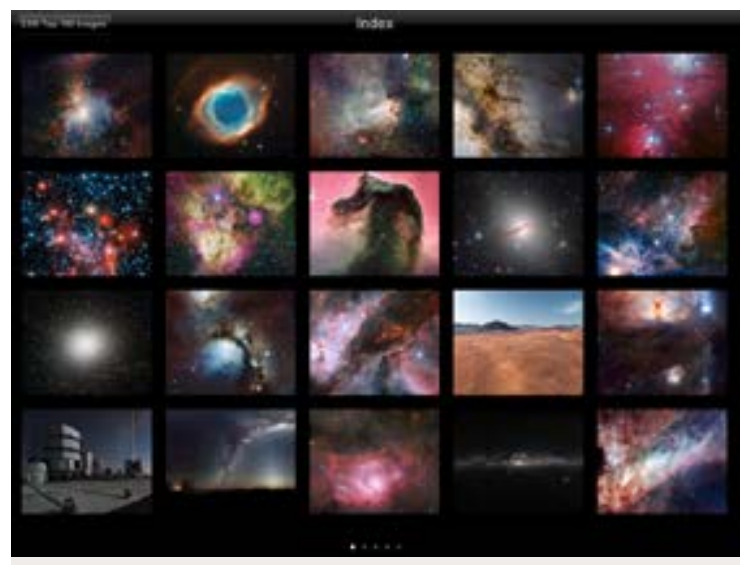

Type de l'infrastructure: $\mathrm{OI}$

\section{Statut de l'infrastructure :}

Organisation Internationale

Localisation de l'infrastructure:

Santiago (CL), La Silla (CL),

le Cerro Paranal (CL), le Cerro Armazones (CL),

plateau de Chajnantor (CL)

\section{Localisation des autres sites :}

Garching (DE)

\section{Représentants de la France :}

Laurent VIGROUX, Denis MOURARD

Tutelles / Partenaires :

CNRS, CEA, ONERA, Observatoire de Paris

\section{Contact en France :}

eson-france@eso.org
L'ESO est la principale organisation intergouvernementale européenne dans le domaine de l'astrophysique au sol; quinze pays européens en sont membres et contribuent en proportion de leur PIB . Courant 2015, le Brésil a ratifié l'accord d'accession au niveau parlementaire et est en attente de la signature présidentielle. Le Chili, pays hôte, n'est pas membre de l'ESO mais bénéficie de $10 \%$ du temps d'observation.

Les programmes scientifiques sont très variés, et vont de la planétologie à la cosmologie. A part la physique solaire et l'exploration directe des corps du système solaire, l'ensemble des grandes questions de l'astronomie sont abordées. Les observatoires dépendant de l'ESO sont les suivants:

- observatoire de La Silla (télescope de 3,60 m et NTT):

- observatoire de Paranal (4 télescopes de 8,20 m du VLT/VLTI, 4 téléscopes de 1,80 m et 2 télescopes grand champ):

- observatoire millimétrique et sub millimétrique ALMA à hauteur de 37,5\%, Amérique du Nord (Etats-Unis et Canada) à hauteur de 37,5\%), Asie (Japon et Taiwan, à hauteur de $25 \%$ ), Chili (pays hôte, pas de contribution directe), comprenant 66 antennes (50 antennes de $12 \mathrm{~m}$ de diamètre pour l'interférométrie, 4 antennes de $12 \mathrm{~m}$ pour des observations en puissance totale, et un réseau compact de 12 antennes de $7 \mathrm{~m}$ de diamètre) :

- E-ELT : projet de télescope dans le proche infrarouge de $39 \mathrm{~m}$ de diamètre, inscrit sur la feuille de route ESFRI.

\section{IMPLICATIONSSOCIO-ÉCONOMIQUES}

La France bénéficie, comme les autres pays membres de l'ESO, d'une partie des contrats liés à la construction et aux opérations des observatoires. La partie la plus importante des retours industriels au niveau local et national se situe lors des phases de développement des télescopes et des instruments avec une particularité forte dans le domaine de l'optique.

\section{DONNÉES}

Estimation du flux de données : Quelques centaines de Go/nuit.

Stockage : Les données acquises par les télescopes sont, après un premier prétraitement automatique sur site, transmises par réseau à Santiago (CL), puis à Garching (DE) où elles sont archivées.

Accessibilité : Les données archivées (aux standards de l'astronomie) sont mises à disposition de l'Investigateur Principal de la proposition et, à l'issue de la période propriétaire d'une année, de toute personne qui en fait la demande. Les métadonnées sont également publiques.

Les métadonnées et les données auxiliaires font partie intégrante de l'archive, accessibles via le répertoire de l'International Virtual Observatory (IVOA).

Présence dans des réseaux de données : International Virtual Observatory (IVOA).

\section{Coût de fonctionnement \\ 151,5 M€/an dont \\ 24,14 M€ pour la France}

\section{Coût RH \\ 730 ETPT (pas de personnel statutaire français)}

\section{Dimension internationale}

Coordinateur : Directeur général de l'ESO : Tim de Zeeuw

Pays partenaires : DE, AT, BE, DK, ES, FI, FR, IT, NL, PL, PT, CZ, UK, SE, CH

Site internet : www.eso.org 


\section{Atacama Large Millimeter/ Submillimiter Array}

Les objectifs principaux d'ALMA sont l'étude du gaz moléculaire et de la poussière dans l'univers. Les principaux thèmes scientifiques que l'on peut mettre en avant sont la formation et l'évolution des galaxies, depuis l'univers lointain à haut décalage spectral jusqu'à l'univers local, la physique et la chimie du milieu interstellaire et la formation des étoiles et des systèmes planétaires, l'étude des comètes et des atmosphères planétaires, ainsi que des petits corps du système solaire.

ALMA est un interféromètre radio comprenant 66 antennes (50 antennes de $12 \mathrm{~m}$ de diamètre pour l'interférométrie, 4 antennes de $12 \mathrm{~m}$ pour des observations en puissance totale, et un réseau compact de 12 antennes de $7 \mathrm{~m}$ de diamètre). La plus grande ligne de base disponible sera de $14 \mathrm{~km}$, permettant d'atteindre une résolution de 0,007 seconde d'arc à la plus haute fréquence observable.

Le temps d'observation est attribué sur appel d'offre ouvert à l'ensemble de la communauté internationale. Les propositions sont évaluées selon leur mérite scientifique par un comité unique d'allocation du temps de télescope; le temps d'observation est ensuite attribué de façon à ce que chaque partenaire ait un retour proportionnel à son investissement, le Chili en recevant pour sa part $10 \%$.

\section{IMPLICATIONSSOCIO-ÉCONOMIQUES}

La France est très impliquée dans la réalisation d'ALMA (antennes : Thales Alenia Space; détecteurs, back end et corrélateur : IRAM) et dans l'analyse des données. La réalisation des 25 antennes de 12 m était un défi technologique en raison des spécifications de masse, de précision (pointage à une fraction de seconde d'arc, surface précise à $25 \mu \mathrm{m}$, stabilité en température, au vent, etc.).

\section{DONNÉES}

Estimation du flux de données : Le flux de données est estimé à 200 To/an en régime de croisière, mais pourrait atteindre 500 To/an.

Stockage : Les données sont archivées et rendues accessibles dans un système compatible OV (Observatoire Virtuel). Les données sont transférées depuis le site d'observation jusqu'à Santiago, puis vers chacun des 3 ALMA Regional Centers.

Accessibilité : Les données sont stockées dans les standards de l'astronomie avec une période propriétaire d'un an. Les métadonnées et les données auxiliaires font partie intégrante de l'archive. L'ensemble des données sera bientôt accessible sur le répertoire de l'International Virtual Observatory Alliance-IVOA.

Présence dans des réseaux de données : International Virtual Observatory

\section{Coût de construction}

\section{-}

\section{1,3 Md€}

Coût de fonctionnement:21 M€/an

\section{Dimension internationale}

ALMA est un instrument de l'Organisation Internationale ESO

Coordinateur : Joint ALMA Observatory (JAO)

Pays partenaires : Consortium ESO, US, JA

Site internet : www.almaobservatory.org

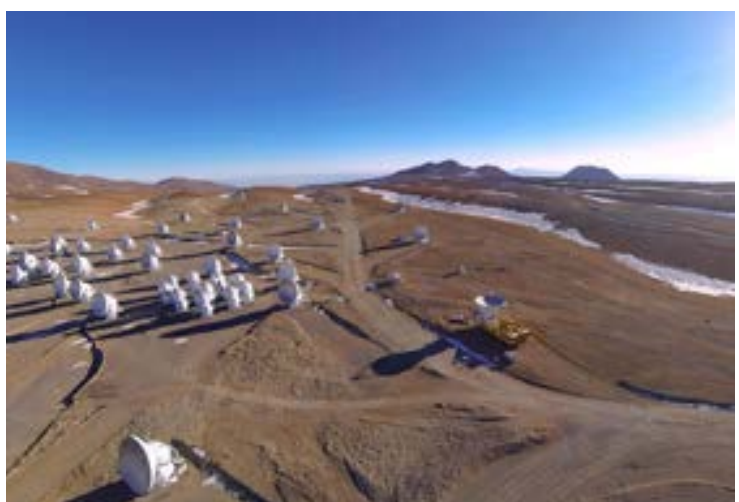

Type de l'infrastructure : Instrument de l'ESO

\section{Localisation:}

plateau de Chajnantor (CL)

\section{Représentant de la France :}

Denis MOURARD

\section{Exploitation:}

2011 (réseau partiel), 2013 (réseau complet)

\section{Contact en France :}

denis.mourard@cnrs-dir.fr

\section{www.eso.org/public/france/} teles-instr/alma 


\section{European Extremely Large Telescope}

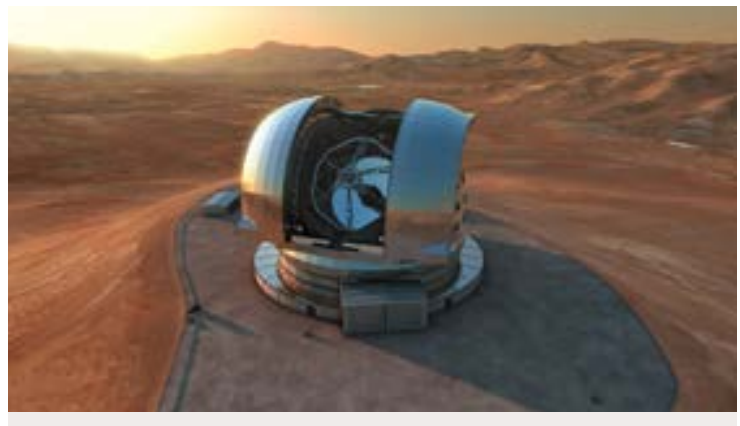

Type de l'infrastructure : Instrument de l'ESO

Localisation de l'infrastructure:

Cerro Armazones(CL)

Représentant de la France :

Denis MOURARD

Création :

Exploitation :

2014

2024

\section{Contact en France :}

denis.mourard@cnrs-dir.fr

\section{www.eso.org/public/teles-instr/ e-elt}

L'E-ELT sera le plus grand télescope au monde (près de 40 mètres de diamètre) dans le domaine de l'observation en lumière visible et infrarouge proche, plus de cent fois plus sensible que les plus grands télescopes optiques actuels. II sera capable de corriger des distorsions atmosphériques et fournira des images 15 fois plus précises que celle du Hubble Space Telescope (HST).

Objectifs scientifiques

- imagerie directe de planètes hors du système solaire et recherche de l'origine de la vie. Cet objectif est central et détermine le diamètre du télescope.

- étude des constituants de l'univers à toutes les échelles : étoiles, galaxies, amas de galaxies.

- étude des premiers objets de l'univers : galaxies, trous noirs, et première percée dans l'âge sombre de l'univers.

L'E-ELT sera complémentaire des très grands observatoires au sol et spatiaux fonctionnant à d'autres longueurs d'onde en 2020 ou au-delà : ALMA depuis 2012 dans le domaine radio millimétrique et sub-millimétrique, et LOFAR (2008) puis SKA (2020+) à plus grande longueur d'onde, le James Webb Space Telescope (NASA/ESA, 2018), télescope infrarouge spatial de 6,5 m de diamètre, et Athena (ESA, 2028), télescope X spatial.

Une coordination a été mise en place par le CNRS/INSU afin de gérer la participation française aux développements des instruments focaux.

L'E-ELT a été affiché comme une priorité lors des deux derniers colloques de prospective en astronomie en France (2009 et 2014). C'est aussi une priorité de la communauté astronomique européenne (première priorité ASTRONET).

\section{IMPLICATIONSSOCIO-ÉCONOMIQUES}

Pour les contrats de phase $B$ de l'E-ELT, le retour industriel français a été de $21 \%$ (12 M€ sur $57 \mathrm{M€}$ au total). Le retour industriel attendu pour la construction de l'E-ELT est de 150 à 200 M€ : segments du miroir M1, polissage du miroir M2, pétales du miroir M4 (SAGEM). A cela s'ajoute la contribution à l'optique adaptative pour les laboratoires, et in fine l'industrie, soit $15 \mathrm{M} €$ supplémentaires.

\section{DONNÉES}

Estimation du flux de données : Quelques centaines de Go par nuit.

Stockage : Les données seront stockées dans les standards de l'astronomie. Les métadonnées et les données auxiliaires feront partie intégrante de l'archive. Accessibilité : Voir la fiche générale de l'ESO.

Présence dans des réseaux de données : International Virtual Observatory.

\section{Coût de construction}

\section{-}

1,104 Md€. La phase 1 est estimée à 997,5 M€

et la phase 2 à 106,5 M€.

Coût de fonctionnement : $45 \mathrm{M€/an,} \mathrm{personnel} \mathrm{ESO} \mathrm{inclus}$

\section{Dimension internationale}

E-ELT est un instrument de I'Organisation Internationale ESO 


\section{La Silla \& Paranal Observatory}

Les programmes scientifiques couverts par le VLT/VLT-I sont très variés, de la planétologie à la cosmologie. Le télescope de 3,60 m est principalement dédié aux exoplanètes.

Le Very Large Telescope (VLT) est un réseau de 4 télescopes de $8 \mathrm{~m}$ de diamètre qui peuvent être opérés soit individuellement,soit simultanément en mode interférométrique (VLTI), ou encore être combinés à 4 télescopes auxiliaires de $1,80 \mathrm{~m}$ de diamètre.

Chaque télescope de $8 \mathrm{~m}$ dispose de 3 instruments focaux pour une utilisation individuelle. La lumière reçue par les télescopes de 8 m et les télescopes auxiliaires peut être recombinée de façon cohérente en mode interférométrique et analysée par l'un des 3 instruments disponibles aujourd'hui, ou en mode incohérent lorsque l'instrument ESPRESSO sera disponible (Pour les instruments focaux du VLT/VLT-I, voir la fiche INSTRUM-ESO).

En mode interférométrique, la résolution spatiale est celle d'un télescope de $200 \mathrm{~m}$ de diamètre environ, correspondant à la plus grande séparation entre les télescopes. Le VLT est unique, non par la taille des télescopes, car il en existe plus d'une dizaine au monde de la classe 8-10 m, mais par la capacité de coupler en mode interférométrique 4 télescopes de la classe $8 \mathrm{~m}$, ce qui donne accès à des possibilités d'imagerie inenvisageables avec seulement 2 télescopes (cas du Keck à Hawaï). Par ailleurs, la qualité du site, la fiabilité des opérations, et un ensemble très complet d'instruments focaux font du VLT le meilleur observatoire mondial pour l'astronomie dans le visible et l'infrarouge proche.

\section{IMPLICATIONS SOCIO-ÉCONOMIQUES}

La France bénéficie, comme les autres pays membres de l'ESO, d'une partie des contrats liés à la maintenance du VLT, mais cette fraction est limitée, compte tenu de l'implantation du VLT. Cela concerne surtout l'optique (notamment l'optique adaptative) et l'optomécanique. Pour l'instrumentation voir la fiche INSTRUM-ESO.

\section{DONNÉES}

Estimation du flux de données : Quelques centaines de Go/nuit

Stockage : Voir la fiche générale de l'ESO.

Accessibilité : Voir la fiche générale de l'ESO.

Présence dans des réseaux de données : International Virtual Observatory.

\section{Coût de fonctionnement \\ 40,4 M€/an}

\section{Dimension internationale}

L'observatoire La Silla-Paranal est l'un des instruments gérés par I'Organisation Internationale ESO.

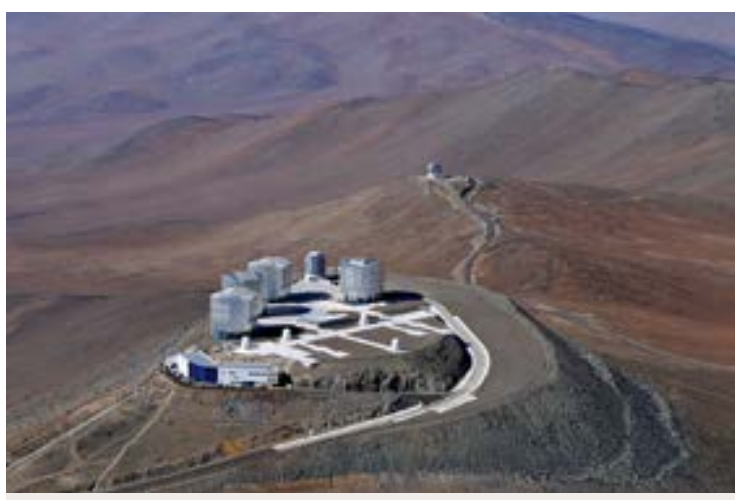

Type de l'infrastructure : Instrument de l'ESO

\section{Localisation:}

Cerro Paranal (CL), La Silla (CL)

Représentant de la France :

Denis MOURARD

\section{Exploitation:}

1998

\section{Contact en France :}

denis.mourard@cnrs-dir.fr

\section{www.eso.org/sci/facilities/}

\section{Ipo.html}




\section{Canada-France-Hawaii Telescope}

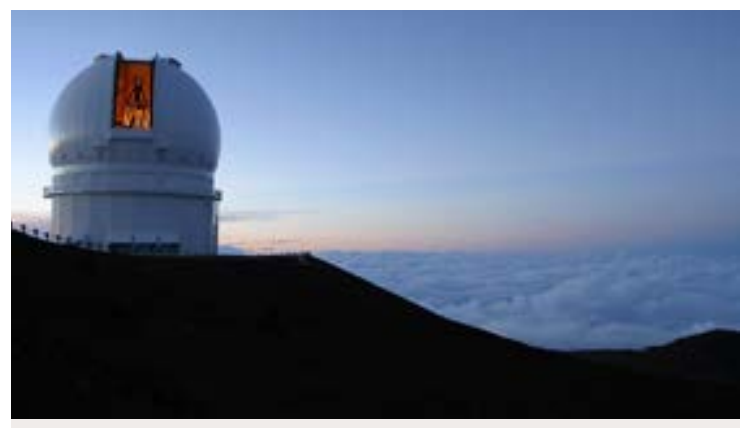

Type de l'infrastructure: TGIR

\section{Statut de l'infrastructure :}

Société à but non lucratif de droit américain

\section{Localisation de l'infrastructure :}

Hawaii

\section{Représentant de la France : \\ Pierre-Olivier LAGAGE \\ Création: Exploitation: \\ 1974

\section{Upgrade :}

L'Instrument SPIRou prévu en 2017

\section{Tutelles / Partenaires :}

CNRS, Paris DIM-ACAV, Idex Toulouse, Univ. Joseph Fourier, Aix Marseille Université

\section{Contact en France :}

pierre-olivier.lagage@cea.fr

\section{www.cfht.hawaii.edu/fr/public}

Le CFHT est situé dans I'un des meilleurs sites de l'hémisphère nord, qui permet des observations de grande qualité, notamment en termes de qualité d'image. Tous les domaines de l'astronomie, y compris la planétologie et la cosmologie, sont concernés.

L'infrastructure est composée d'un télescope de 3,6 m et d'une suite d'instruments très performants associés: MEGACAM (imagerie à grand champ dans le domaine visible), WIRCAM (imagerie dans le domaine infrarouge), ESPaDOnS (spectropolarimètre pour l'étude de la vie magnétique des étoiles), Sitelle (un spectromètre à transformée de Fourier dans le domaine visible) et bientôt (en 2017) SPIRou (un spectro-polarimètre infrarouge ultra stable).

Le temps d'observation est attribué à partir d'appels à propositions évaluées et classées par un comité scientifique. Une très large fraction du temps d'observation (60\%) est consacrée à des "larges programmes», avec des configurations instrumentales stables, des observations en mode service et tout récemment l'introduction d'une estimation en temps réel du rapport signal à bruit afin d'optimiser la durée des poses. Une part importante du temps de télescope devrait être consacrée dans les prochaines années à deux programmes majeurs

- la cartographie d'une grande partie du ciel boréal avec la caméra MEGACAM pour étudier les galaxies et apporter un complément indispensable aux observations de la mission spatiale Euclid d'étude de l'énergie noire; - l'étude d'exoplanètes à partir d'observations spectroscopiques dans I'infrarouge avec l'instrument SPIRou.

\section{IMPLICATIONSSOCIO-ÉCONOMIQUES}

Une instrumentation innovante à la pointe de la technologie est nécessaire et demande souvent des études amont de R\&D. On peut citer notamment les détecteurs Hawaii 4RG pour l'instrument SPIRou ou encore les grands réseaux pour les spectrographes à échelle montés sur ESPaDOnS et SPIRou.

\section{DONNÉES}

Estimation du flux de données : 30 To/an

Stockage : Centre canadien de données astronomiques (CADC). Les données du programme CFHTLS ont été analysées au centre TERAPIX de I'IAP (http://terapix.iap.fr).

Accessibilité : Données accessibles au format international FITS (répertoire IVOA au CADC) à la fin de la période propriétaire.

Présence dans des réseaux de données : IVOA

\section{Coût de construction \\ - \\ 4,1 M€pour l'instrument SPIRou dont 1,8 M€ de contribution française Coût de fonctionnement : 7,2 M\$, dont 3,2 M\$ pour la France}

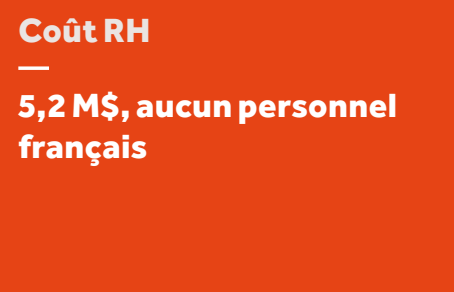

Dimension internationale

Coordinateur : Douglas Welch

Pays partenaires : FR, CA, US 


\section{Institut de RadioAstronomie Millimétrique}

L'IRAM est un institut international (France-Allemagne-Espagne) chargé de développer, faire fonctionner, et mettre à disposition de la communauté scientifique deux observatoires dans le domaine des longueurs d'onde millimétriques et submillimétriques (de 0,8 à 3,4 mm, soit 70 à $350 \mathrm{GHz}$ ).

Les instruments de I'IRAM offrent des possibilités d'observations extrêmement vastes dans le domaine (sub)millimétrique, un des domaines clés de l'astrophysique moderne. Les thématiques scientifiques couvrent notamment l'étude du système solaire (atmosphères planétaires, comètes), la formation des étoiles et des disques protoplanétaires, les phases évoluées des étoiles, les propriétés physico-chimique du milieu interstellaire, celles des galaxies proches, mais aussi la formation des galaxies dans l'univers à grand redshift et les études cosmologiques associées.

Les deux observatoires de I'IRAM sont:

- une antenne de 30 m de diamètre située sur le Pico Veleta, dans le massif de la Sierra Nevada, près de Grenade (Andalousie):

- un interféromètre composé de 7 antennes de 15 m de diamètre (en 2015), situé sur le Plateau de Bure, dans le massif du Devoluy (Hautes-Alpes), qui passera progressivement à 12 antennes en 2019 (projet NOEMA).

L'IRAM dispose d'équipes et de laboratoires couvrant un très large ensemble de besoins techniques et scientifiques : récepteurs, fabrication d'éléments supra-conducteurs, électronique, mécanique de haute précision, logiciels de commande, logiciels de réduction de données, etc.

\section{IMPLICATIONSSOCIO-ÉCONOMIQUES}

Une large partie des dépenses pour NOEMA sont des achats de composants de haute technologie, dont le développement nécessite des efforts importants de R\&D. Des projets de développements spécifiques sont en cours avec plusieurs entreprises dans le domaine de l'électronique rapide $(\mathrm{THz})$, de la cryogénie, de la mécanique de haute précision, ou de la réalisation de systèmes de contrôle intégrés.

\section{DONNÉES}

Estimation du flux de données : Flux de données de NOEMA : $400 \mathrm{Go}$ à 3 To/jour en fonction du mode d'observation.

Stockage : Archivage des données à l'IRAM.

Accessibilité : Données accessibles immédiatement aux équipes ayant proposé les observations; données libres après période propriétaire de 18 mois à 3 ans.

Présence dans des réseaux de données : CDS et IVOA

\section{Coût de fonctionnement \\ $11 \mathrm{M} € /$ an frais \\ de personnel compris}

\section{Dimension internationale \\ Coordinateur : Karl Schuster \\ Pays partenaires : $F R, D E, E S$}

Site internet : www.iram-institute.org

\section{ETPT dont 90}

en France

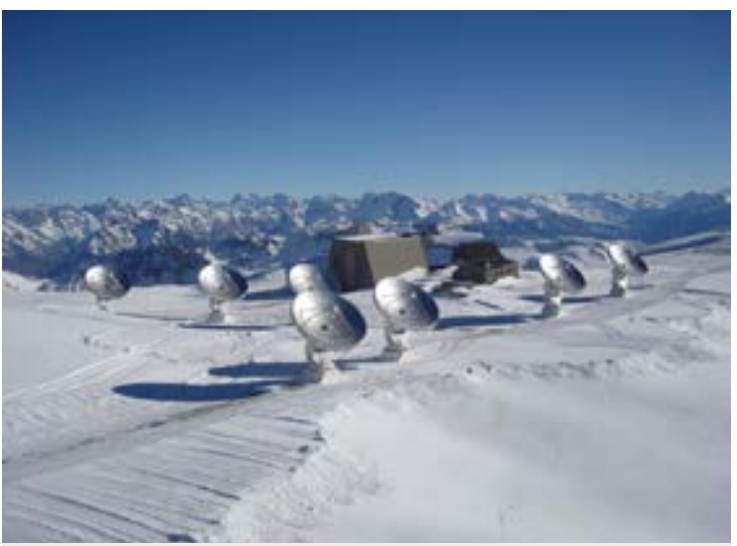

Type de l'infrastructure: TGIR

\section{Statut de l'infrastructure :}

Société civile de droit français

\section{Localisation :}

Siège à Grenoble

\section{Localisation des autres sites :}

Plateau de Bure, Grenade (ES), Pico Veleta (ES)

\section{Responsable de l'infrastructure :}

Karl-Friedrich SCHUSTER

\section{Création :}

1979

\section{Exploitation :}

1986 (Pico Veleta), 1990 (Plateau de Bure)

\section{Upgrade :}

Projet NOEMA : passage de 6 à 12 antennes de 2014 à 2019

\section{Tutelles / Partenaires :}

CNRS (FR, $47 \%$ ), Max-Planck-Gesellschaft

(DE, $47 \%$ ), Instituto Geográfico Nacional

(ES, 6\%)

\section{Contact en France :}

schuster@iram.fr

\section{www.iram-institute.org}




\section{Centre de Données astronomiques de Strasbourg}

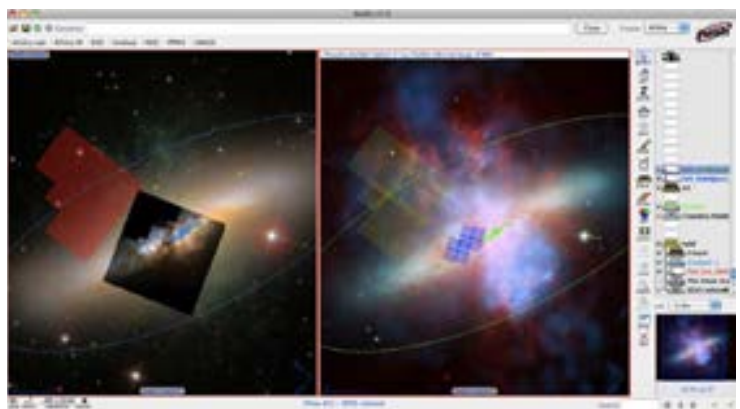

Type de l'infrastructure : IR

Statut de l'infrastructure :

Unité mixte de recherche

Localisation de l'infrastructure:

Strasbourg

Localisation des autres sites :

Infrastructure virtuelle

Responsable de l'infrastructure :

Mark ALLEN

\section{Exploitation :}

1972

Tutelles / Partenaires :

CNRS, Univ. Strasbourg

\section{Contact en France :}

mark.allen@astro.unistra.fr

\section{cds.unistra.fr}

La mission du CDS est de collecter, homogénéiser, distribuer l'information astronomique, pour le bénéfice de l'ensemble de la communauté internationale. L'objectif est de faciliter le travail des astronomes en regroupant de l'information dans ses services, avec des liens avec les archives d'observatoires, les journaux académiques et les autres bases de données, en particulier ADS et NED. Le CDS est l'un des acteurs majeurs du développement de l'Observatoire Virtuel (OV) astronomique, qui vise à donner un accès transparent à l'ensemble des ressources en ligne de l'astronomie.

Le CDS développe des services largement utilisés par la communauté : SIM$B A D$, la base de données de référence pour les identifications et la bibliographie des objets hors système solaire; VizieR, la base de données de référence pour les grands relevés du ciel, les catalogues et les tables publiées dans les journaux académiques, et de plus en plus pour d'autres types de données attachées aux publications; l'atlas interactif du ciel Aladin, portail qui permet d'accéder à la collection d'images de référence du CDS et aux images disponibles dans les archives des observatoires sol et spatiaux: s'y ajoute depuis 2011 le meilleur service d'identification croisée de très grands catalogues.

En 2014, les services ont généré plus de 800000 requêtes par jour, 679 articles dans des revues à lecteurs ont cité le mot SIMBAD, 306 VizieR et 58 Aladin (source ADS).

\section{IMPLICATIONS SOCIO-ÉCONOMIQUES}

Collaboration avec les éditeurs scientifiques et les publishers des journaux académiques de la discipline pour enrichir les publications de liens vers les données. Dès 1993, le CDS a mis en ligne sur internet les tables publiées dans le journal Astronomy \& Astrophysics.

\section{DONNÉES}

Estimation du flux de données : Le CDS fournit des services permettant d'accéder à des données et de les exploiter. Ces données incluent celles qu'il met lui-même à disposition, mais aussi celles des archives des observatoires via des liens et l'interopérabilité de l'Observatoire Virtuel.

Stockage : Environ 1 Po

Accessibilité : Les données et les services sont en libre accès.

Présence dans des réseaux de données : Les services sont disponibles via l'Observatoire Virtuel. Le CDS est membre du World Data System (www.icsu-wds.org) de l'ICSU et a acquis la certification Data Seal of Approval (www.datasealofapproval.org/en).

\section{Coût de fonctionnement}

$0,7 \mathrm{M} €$

\section{Coût RH}

\section{Dimension internationale}

Le CDS porte depuis 2006 la coordination de l'Observatoire Virtuel européen Euro-VO. Celui-ci est membre de IVOA.

Coordinateur : IVOA (International Virtual Observatory Alliance)

Pays partenaires : Euro-VO : FR, DE, ES, IT, UK

IVOA : ZA, DE, AR, AM, AU, BR, CA, CL, CN, ES, FR, HU, IN, IT, JP, UK, RU, UA, US

Site internet : www.ivoa.net 


\section{INSTRUM-ESO}

\section{Instrumentation pour les grands télescopes de l'ESO}

Les deux instruments VLT de $2^{e}$ génération que la France a portés comme PI, MUSE (spectrographe intégral de champ visible dédié à l'étude de la formation et de l'évolution des galaxies) et SPHERE (détection et caractérisation d'exoplanètes géantes par imagerie à haut contraste dans le visible et I'IR proche) ont été intégrés avec succès à Paranal en 2014 et 2015

La France est aujourd'hui impliquée dans les deux instruments de $2^{e}$ génération du VLT-I comme responsable de l'un et co-responsable de l'autre. II s'agit respectivement de MATISSE qui, en recombinant les 4 télescopes dans I'IR moyen (3-10 $\mu \mathrm{m}$ ), permettra l'obtention d'images avec une résolution de 10 à 20 milliseconde d'arc (principalement pour l'étude de la formation stellaire), et de GRAVITY qui aura une précision astrométrique de 10 microsecondes d'arc sur des objets faibles (tests de relativité générale et étude des exoplanètes). GRAVITY sera installé à Paranal à partir de l'automne 2015 et MATISSE à partir de mi-2016. La France contribue également à la réalisation de l'instrument MOONS, spectrographe à grand champ multi objets destiné aux études galactiques, extragalactiques et cosmologiques.

Les travaux préparatoires pour le futur E-ELT portent sur l'optique adaptative et les instruments focaux. La France prendra une participation importante aux instruments de première lumière HARMONI, MICADO et METIS ainsi qu'à l'optique adaptative (MAORY); elle ambitionne par ailleurs d'être porteur de l'instrument MOSAIC pour lequel un complément de phase A est prévu en 2016/2017

\section{IMPLICATIONSSOCIO-ÉCONOMIQUES}

Les secteurs principalement concernés sont ceux de l'optique de pointe (composants innovants, traitements de surface particuliers, concepts et composants de systèmes d'optique active et adaptative), des détecteurs visibles et infrarouges (grande sensibilité, lecture rapide, grande surface de détection), des calculateurs en temps réel, etc.

\section{DONNÉES}

Présence dans des réseaux de données : Les données astrophysiques produites par les instruments sont gérées par l'ESO (voir fiche générale ESO).

Mots clé : Astronomie, astrophysique, observatoire, ESO, instruments focaux, optique

\section{Coût de fonctionnement \\ environ $20 \mathrm{M€/an}$}

\section{Coût RH \\ $-$ 35 ETPT}

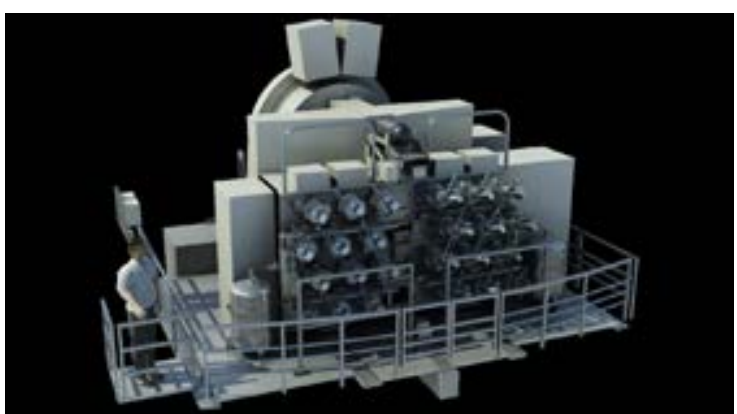

Type de l'infrastructure : IR

\section{Localisation :}

Paris

\section{Localisation des autres sites :}

IR distribuée

Responsable de l'infrastructure: Denis MOURARD

\begin{tabular}{|r|c|}
\hline Création : & Exploitation : \\
\hline 2015 & 2015 \\
\hline
\end{tabular}

\section{Tutelles / Partenaires :}

CNRS, CEA, ONERA

\section{Contact en France :}

denis.mourard@cnrs-dir.fr

\section{www.eso.org/public/france/}

about-eso

\section{Dimension internationale \\ L'infrastructure contribue directement à la réalisation des moyens instrumentaux de l'ESO. La coordination nationale interagit très directement avec l'ESO ainsi qu'avec le réseau de plateformes d'essai et de développement en cours de mise en place.}

Coordinateur : ESO (Organisation Internationale)

Site internet: www.eso.org 


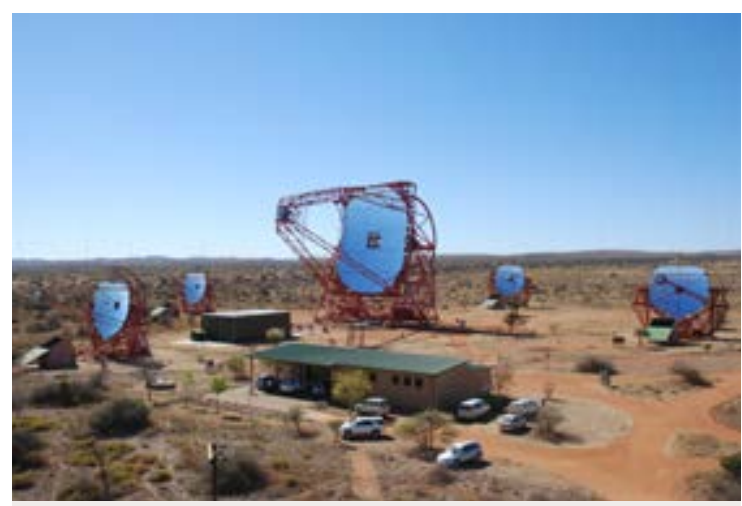

Type de l'infrastructure : IR

\section{Localisation de l'infrastructure :}

Khomas Highlands, Namibie

Responsable de l'infrastructure :

Mathieu DE NAUROIS

\section{Exploitation :}

2003

\section{Maintenance :}

Les caméras des quatre premiers télescopes font actuellement l'objet d'une remise à niveau complète. Le projet CTA est un successeur de HESS.

Tutelles / Partenaires :

CNRS, CEA

\section{Contact en France :}

denauroi@in2p3.fr

\section{polywww.in2p3.fr/HESS}

Lors de leur entrée dans l'atmosphère terrestre, les rayons cosmiques produisent une gerbe de particules qui, se déplaçant à une vitesse supérieure à celle de la lumière dans l'air, émettent un rayonnement Cherenkov. Celui-ci est détecté par un réseau de télescopes au sol de grande surface collectrice, disposés à l'intérieur d'un carré de 120 m de côté. Ce dispositif permet d'identifier l'origine et l'énergie de chaque rayon gamma. Le réseau est composé de 4 télescopes d'un diamètre de $12 \mathrm{~m}$ et d'un cinquième télescope de $28 \mathrm{~m}$ de diamètre, inauguré en septembre 2012. Ce dernier permet de doubler la sensibilité du réseau et d'abaisser le seuil en énergie (jusqu'à $50 \mathrm{GeV}$ voire $20 \mathrm{GeV}$ ) pour étendre le champ d'étude des sources de rayonnement et améliorer le recouvrement avec le satellite Fermi sources galactiques (pulsars, centre de la galaxie, restes de supernova), objets extragalactiques (noyaux actifs de galaxie, sursauts gammas) ou sources exotiques, telles que le halos galactique, les amas globulaires ou les galaxies naines, qui pourraient révéler en gamma l'annihilation de particules de matière noire ( WIMPS »).

HESS est utilisé pour la recherche en astrophysique des hautes énergies, notamment les mécanismes d'émissions des sources galactiques et extragalactiques, et la cartographie à haute résolution spatiale du ciel austral.

\section{IMPLICATIONSSOCIO-ÉCONOMIQUES}

La construction a fait appel à de nombreuses entreprises spécialisées dans les domaines du génie civil, de la mécanique (structures métalliques), de la microélectronique (ASICs), de l'automatisme et de la photo-détection.

\section{DONNÉES}

Estimation du flux de données : 1 Po, en croissance de 150 à 200 To/an.

Stockage : Les données sont stockées sur site, puis envoyées (en deux copies distinctes) en France (au centre de calcul CNRS de Lyon) et en Allemagne (Heidelberg) sous forme de bandes magnétiques.

Accessibilité : Les données appartiennent à la collaboration. II n'existe pas à ce jour d'outil « public » permettant d'accéder à ces données et de les interpréter. L'archive utilise le format ROOT (root.cern.ch) développé au CERN et largement utilisé dans la communauté de physique des hautes énergies. L'accès aux données se fait à travers des protocoles standardisés développés pour cette même communauté (xrootd, hpss, LFC...).

Présence dans des réseaux de données : Centre de calcul CNRS de Lyon, Grille de calculEGI

\section{Coût de construction \\ 20,5 M€ dont 6,5 M€ \\ pour la France \\ Coût de fonctionnement : \\ $2 \mathrm{M} € /$ an dont 0,5 M€ \\ pour la France}

\section{Dimension internationale \\ Coordinateur: Mathieu de Naurois, FR \\ Pays partenaires : FR, DE, NA, ZA, UK, IE, AT, PL, CZ, SE, AM, AU, JP \\ Site internet : www.mpi-hd.mpg.de/hfm/HESS}




\section{ILT-LOFAR FR}

\section{International Low Frequency Radio Array Telescope - LOFAR FR}

LOFAR permet d'étendre les observations radio aux plus basses fréquences et à la plus haute résolution angulaire accessibles depuis le sol. C'est le premier grand radiotélescope "numérique», dont les opérations et les performances reposent sur le transport et le traitement à très haut débit du signal de milliers d'antennes. Ses champs d'application scientifique principaux sont la cosmologie, les amas de galaxies, les champs magnétiques cosmiques, le rayonnement cosmique, le Soleil, les planètes, et l'univers variables (pulsars, trous noirs et sources à haute énergie, planètes et exoplanètes). Chaque station internationale comprend 96 antennes (de 2 dipôles pour donner les 4 paramètres de polarisation) basse-fréquence et 96 tuiles d'antennes haute-fréquence, reliées à très haut débit $(3 \mathrm{~Gb} / \mathrm{s})$ au corrélateur central à Gröningen (NL).

NenuFAR en étendra fortement la sensibilité et d'autres caractéristiques comme un étalonnage amélioré; les antennes et l'électronique de NenuFAR sont particulièrement optimisées pour l'ensemble de la bande 10-80 $\mathrm{MHz}$. Grâce à un dédoublement du signal des antennes avant l'entrée dans les chaînes de traitement, NenuFAR opèrera à la fois et indépendamment comme une "superstation» de LOFAR (alternative aux antennes basse-fréquence existantes) et comme un instrument autonome avec une programmation scientifique spécifique.

\section{DONNÉES}

Estimation du flux de données : 2 To/jour (100 fois plus pour des données non-compressées).

Stockage : LOFAR Long Term Archive (LTA): équipé d'une interface unique et uniforme permettant d'accéder à des données acquises par les différents équipements de l'ILT, et stockées dans différents endroits (Amsterdam, Groningen, Jülich et un nouveau centre en Pologne).

Accessibilité : Tous les produits de données calibrés résultant des observations du projet ILT ou les données produites par la pipeline ILT restent la propriété de l'ILT. L'ILT accorde au groupe de travail (Proposant et PI) un temps d'accès exclusif aux données d'une durée d'un an, puis les données sont rendues publiques.

Présence dans des réseaux de données : LOFAR participe, en tant que "pathfinder » de SKA, au Work Package Data Access, Discovery and Interoperability du Cluster européen ASTERICS, avec pour objectif d'optimiser l'accès aux données dans l'Observatoire Virtuel.

\section{Coût de fonctionnement $0,112 \mathrm{M} €(F R)$}

\section{Coût RH \\ - \\ LOFAR : 0,6 ETPT; \\ NenuFAR 4 ETPT pendant la phase de construction}

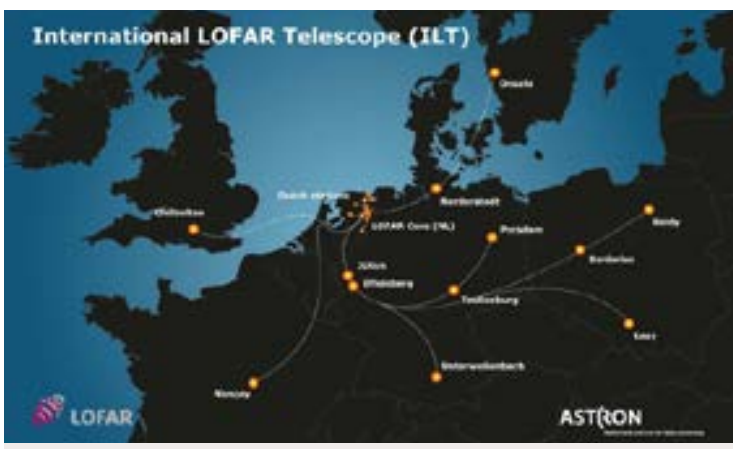

Type de I'infrastructure : IR

Statut de l'infrastructure :

Fondation de droit néerlandais

\section{Localisation :}

Nançay

\section{Localisation des autres sites :}

Autres stations : NL (37), DE (6), UK (1), SE (1),

PL (3), IE (1)

Responsables de l'infrastructure: Michel TAGGER, Philippe ZARKA

\begin{tabular}{|r|c|}
\hline Création : & Exploitation : \\
\hline 2010 & 2011 \\
\hline
\end{tabular}

\section{Maintenance:}

2016 : extension NenuFAR

\section{Tutelles / Partenaires :}

CNRS, Observatoire de Paris, Univ. Orléans

\section{Contact en France :}

michel.tagger@cnrs-orleans.fr

\author{
Dimension internationale \\ Participation française à une infrastructure de recherche internationale ILT \\ reconnue comme " pathfinder » du futur projet SKA \\ Coordinateur : ASTRON (NL) \\ Pays partenaires : NL, DE, UK, FR, SE, PL, IE \\ Site internet : $\underline{w w w . l o f a r} .0 r g$
}




\section{CTA}

\section{Cherenkov Telescope Array}

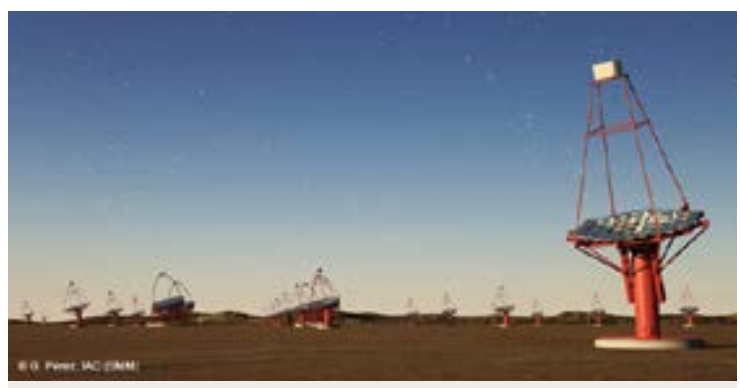

Type de l'infrastructure : PROJET

\section{Statut de l'infrastructure :}

$\mathrm{GmbH}$ (statut provisoire) basée à Heidelberg (DE)

\section{Localisation de l'infrastructure :}

Paranal (CL), La Palma (ES)

\section{Responsable de l'infrastructure :} Jürgen KNODLSEDER

\section{Création :}

Création de la GmbHen 2014, début de construction prévu en 2016-2017

\section{Exploitation :}

2018-2019

\section{Maintenance :}

Mise à niveau tous les 10 ans

\section{Tutelles / Partenaires :}

CEA, CNRS

\section{Contact en France :}

jurgen.knodlseder@irap.omp.eu tabegg@lsw.uni-heidelberg.de

\section{Site web en construction}

\section{www.facebook.com/CTA.France}

Les télescopes Cherenkov observent les photons gamma de haute énergie de manière indirecte en détectant les éclairs ténus de lumière Cherenkov qui sont émis par les gerbes de particules créées lors de l'interaction d'un photon gamma cosmique avec l'atmosphère terrestre. Après le succès des télescopes Cherenkov de seconde génération pour l'astronomie gamma à très haute énergie (et très notamment HESS, collaboration principalement franco-allemande), CTA, avec une centaine de télescopes Cherenkov au sol distribués en deux réseaux, constitue une extension naturelle des télescopes existants

CTA permettra d'accroître la sensibilité des observatoires actuels de plus d'un ordre de grandeur tout en assurant une meilleure résolution angulaire. Les réseaux de télescopes de différentes tailles permettront également d'étendre le domaine d'énergie et le recouvrement à basse énergie avec le satellite Fermi de la Nasa lancé en 2008 et opérationnel pendant 5-10 ans.

\section{IMPLICATIONSSOCIO-ÉCONOMIQUES}

CTA crée des opportunités pour le transfert technologique. Par exemple, le développement des miroirs pour les télescopes de taille moyenne par le CEA/IRFU a donné lieu à un transfert technologique vers la PME Kerdry dans le contexte d'un partenariat avec la région Bretagne. La réalisation des arches des télescopes de grande taille se fait en partenariat avec la PME LORIMA, en région Bretagne.

\section{DONNÉES}

Estimation du flux de données : $6 \mathrm{Go} / \mathrm{s}$ pour environ 1300 heures d'observation annuelles, et environ $3 \mathrm{Po} /$ an après réduction, donc 90 Po pour un temps d'exploitation de 30 ans. Après reconstruction des événements et réjection du bruit de fond, le volume de données annuel est réduit à <1 To.

Stockage : Les données CTA seront archivées de manière parallèle dans plusieurs centres de données. L'accès aux archives se fera à la fois par une interface web et par les registres de l'Observatoire Virtuel (IVOA).

Accessibilité : Données libres d'accès sous format international FITS après un an d'embargo environ. Mise à disposition de logiciels d'exploitation " open source ".

Présence dans des réseaux de données : Il est envisagé de faire appel à des centres de données existants pour le traitement et le stockage des données CTA. La participation du Centre de Calcul CEA-CNRS de Lyon est à l'étude.

\section{Coût de construction \\ Environ 300 M€ dont 50 M€ pour la France (non encore décidée) Coût de fonctionnement:20M€}

\section{Dimension internationale \\ Participation française à une infrastructure internationale de la feuille de route ESFRI (gGmbH à Heidelberg, DE). \\ Coordinateur : Président : G. Vettolani, IT \\ Pays partenaires : DE, ES, IR, CZ, UK, CH, Membre associés : NL, SA, SE, FR (en projet) \\ Site internet : www.cta-observatory.org}


ASTRONOMIE ET ASTROPHYSIQUE 


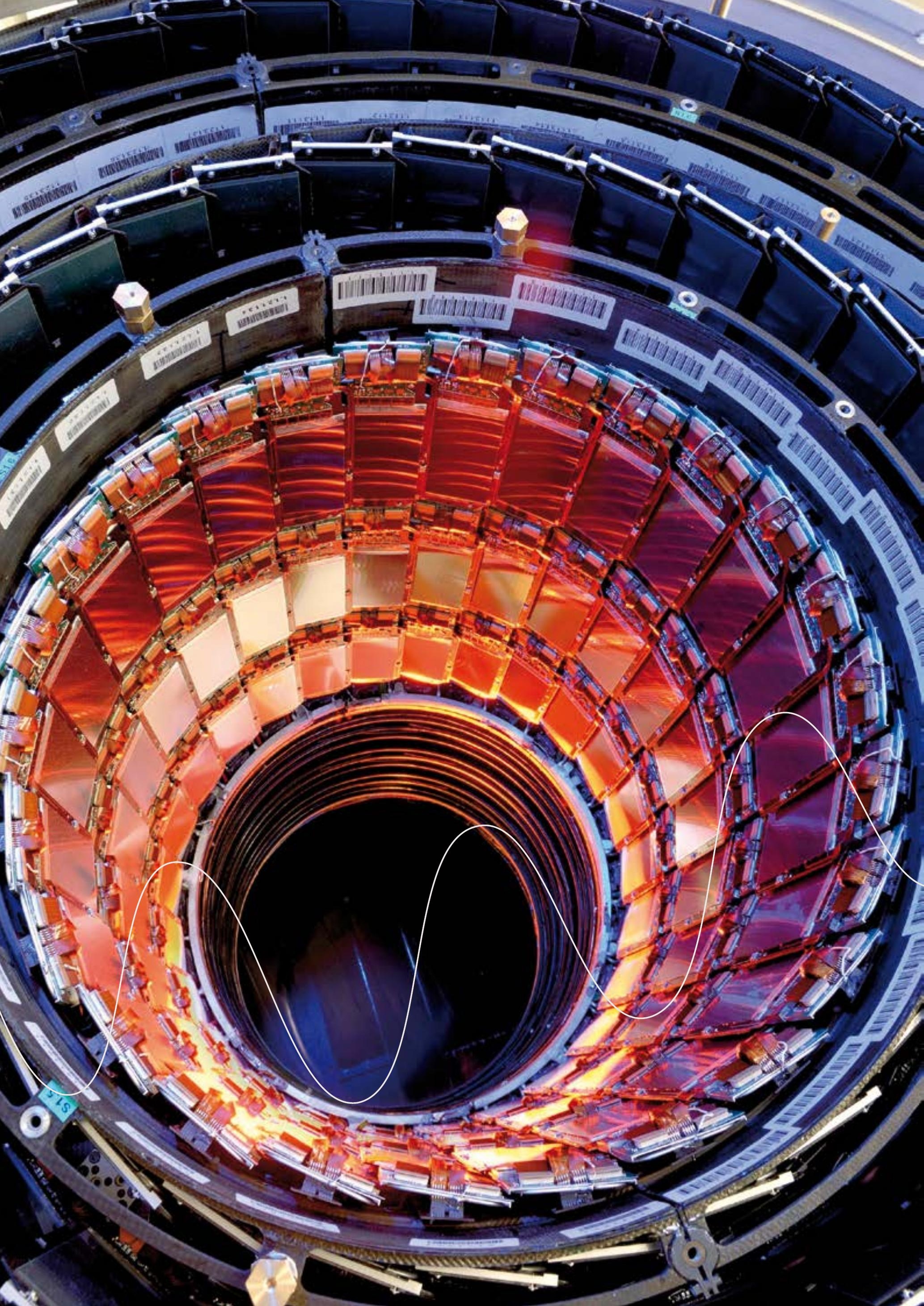


PHYSIQUE NUCLÉAIRE ET DES HAUTES ÉNERGIES

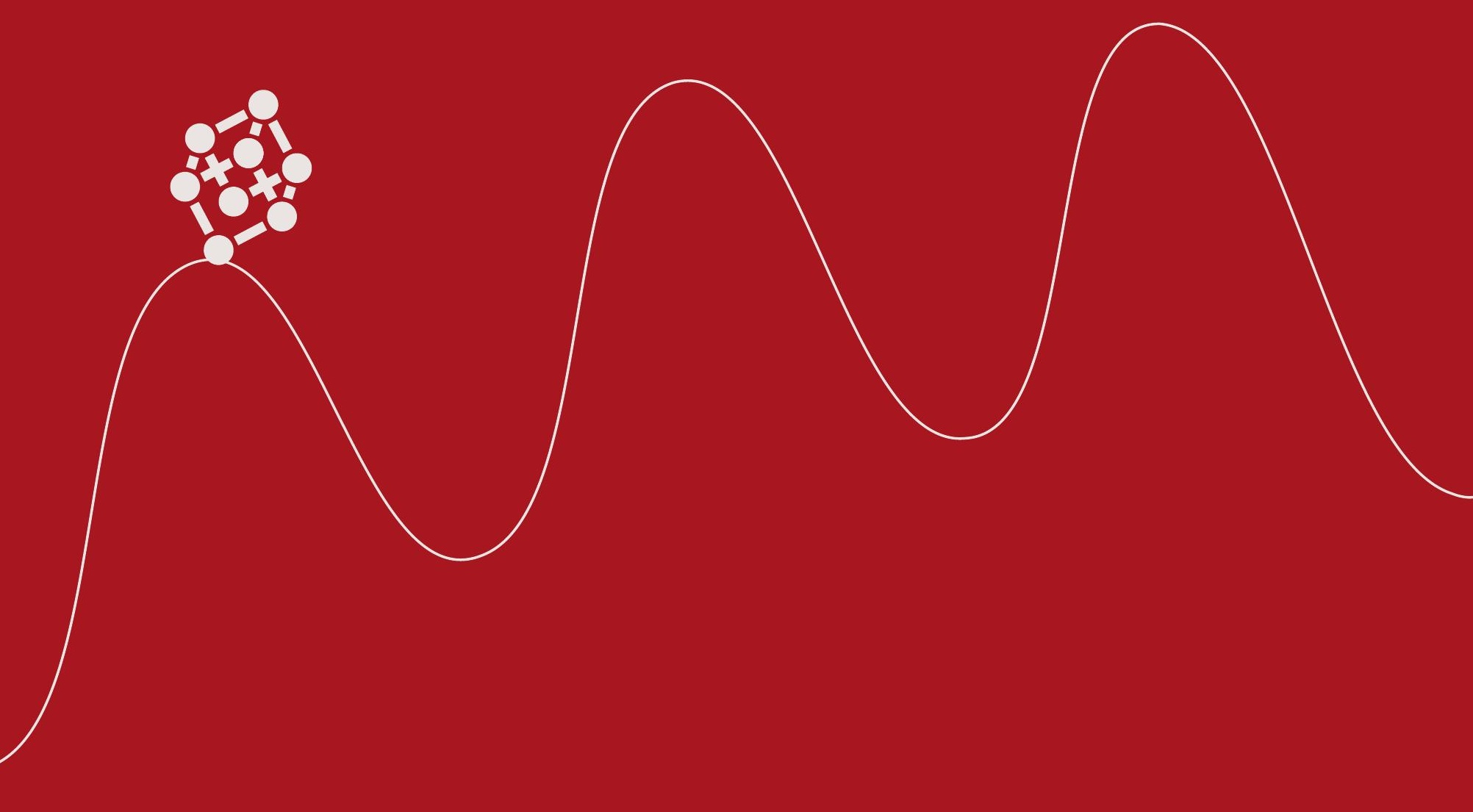




\section{Physique nucléaire et des hautes énergies}

Les grandes infrastructures de recherche de ces domaines scientifiques répondent aux besoins de la communauté des physiciens qui ont pour ambition d'étudier les constituants ultimes de la matière. Ces infrastructures relèvent du Comité de Coordination Thématique TGIR créé par le CEA et le CNRS. En physique nucléaire les scientifiques français sont majoritairement engagés, en France et en Europe, dans les complexes d'accélérateurs d'ions lourds localisés sur les sites du GANIL, à Caen, et de GSI, à Darmstadt en Allemagne délivrant des faisceaux stables et radioactifs. Ces sites ont ainsi vocation à abriter, à l'orée 2017-2025, les fers de lance de la recherche en physique nucléaire européenne, SPIRAL2 et FAIR.

Les objectifs de SPIRAL2 ont été réorientés pour exploiter le plus rapidement possible les Equipex S3 et DESIR, dans un contexte budgétaire particulièrement tendu. SPIRAL2 sera aussi une source de neutrons rapides (14 MeV) parmi les plus performantes au monde pour les dix prochaines années. Quant à FAIR, malgré le retard pris par le projet, il sera unique par l'importance des domaines scientifiques couverts, par la variété des faisceaux accélérés (ions lourds, antiprotons), sa complémentarité avec SPIRAL2 pour les faisceaux radioactifs, et avec des expériences du LHC au CERN et de RHIC et JLAB aux Etats Unis en physique hadronique. SPIRAL2 et FAIR sortiront en 2016 de la feuille de route ESFRI, acquérant un statut de "Landmark " ESFRI. Aujourd'hui, le financement de la phase 2 de SPIRAL2 n'est pas acquis. II passera très probablement par une ouverture du GANIL à d'autres pays.

Après la découverte du boson de Higgs (prix Nobel 2013), l'étude fine de ses propriétés ainsi que les tests de cohérence du modèle standard et la recherche de signaux de physique au-delà du modèle standard sont les toutes premières priorités de la discipline. La stratégie européenne de la physique des particules, approuvée en 2013 par le conseil du CERN, énonce en conséquence comme toute première priorité l'exploitation maximale du LHC avec une première phase d'amélioration des détecteurs qui devront être remis en service vers 2018-2019, et une deuxième phase à haute luminosité qui débutera vers 2026. La prise de données au LHC devrait se poursuivre jusqu'en 2035-2038.
Au vu du temps nécessaire pour concevoir de nouvelles infrastructures en physique des particules, développer les technologies nécessaires et réaliser leur construction, le CERN a lancé dès à présent une étude pour le design d'un futur complexe d'accélérateurs, le «Future Circular Collider » (FCC) qui pourrait héberger des collisionneurs d'une circonférence de $100 \mathrm{~km}$ et la construction d'un collisionneur linéaire est en discussion au Japon.

La découverte des oscillations des neutrinos (prix Nobel 2015) et plus récemment la mesure du dernier angle de mélange 13 , met en lumière les questions fondamentales concernant la hiérarchie des masses des neutrinos et la violation de symétrie $C P$ dans le secteur des neutrinos. En France, le projet ORCA, basé sur la mesure des neutrinos atmosphériques fait suite à l'expérience Antares en Méditerranée, et se déploie dans le cadre de la collaboration internationale $\mathrm{KM} 3 \mathrm{NeT}$ qui comprend aussi un deuxième site près de Catane en Italie dédié à l'astronomie neutrino. A noter que des équipes françaises sont impliquées dans des projets d'étude des neutrinos utilisant d'autres infrastructures en Chine et aux Etats-Unis.

La cosmologie observationnelle, à travers en particulier l'étude du rayonnement fossile, de la matière noire et de l'énergie noire, a obtenu des résultats spectaculaires durant cette dernière quinzaine d'années (prix Nobel 2011, mission Planck). Les projets phares de mesure de la matière noire et de l'énergie noire actuellement en phase de construction sont LSST au sol, et Euclid (mission ESA) dans l'espace. HESS et à terme le projet CTA permettront des observations indirectes de la matière noire.

La découverte des ondes gravitationnelles, annoncée en février 2016 par les collaborations LIGO (USA) et VIRGO à partir de données recueillies par LIGO, va avoir un impact considérable et ouvre une nouvelle fenêtre sur l'Univers. La remise en service de l'antenne VIRGO près de Pise (Advanced Virgo), après un important programme d'amélioration, devrait permettre d'autres détections directes dans les toutes prochaines années. 


\section{LISTE DES INFRASTRUCTURES DE RECHERCHE}

DANS LE DOMAINE PHYSIQUE NUCLÉAIRE ET HAUTES ÉNERGIES

\begin{tabular}{|c|c|c|c|}
\hline CATÉCORIE & NOM & NOM COMPLET & ESFRI \\
\hline OI & CERN-LHC & $\begin{array}{l}\text { Organisation Européenne pour la Recherche } \\
\text { Nucléaire - Grand Collisionneur Hadronique/ } \\
\text { European Organization for Nuclear Research - } \\
\text { Large Hadron Collider }\end{array}$ & \\
\hline TGIR & EGO-VIRGO & $\begin{array}{l}\text { Observatoire Européen Gravitationnel -VIRGO/ } \\
\text { European Gravitational Observatory - VIRGO }\end{array}$ & \\
\hline TGIR & FAIR & Facility for Antiproton and Ion Research & FAIR (2006) \\
\hline TGIR & GANIL-Spiral2 & $\begin{array}{l}\text { Grand Accélérateur National d'lons Lourds - } \\
\text { Système de production d'Ions Radioactifs en Ligne } \\
\text { de } 2^{\mathrm{e}} \text { génération }\end{array}$ & Spiral2 (2006) \\
\hline IR & KM3NeT & Kilometre Cube Neutrino Telescope & $\begin{array}{l}\text { KM3NET (2006, } \\
\text { 2016) }\end{array}$ \\
\hline IR & LSST & Large Synoptic Survey Telescope & \\
\hline IR & HESS $^{1}$ & High Energy Stereoscopic System & \\
\hline Projet & $C T A^{2}$ & Cherenkov Telescope Array & CTA (2008) \\
\hline
\end{tabular}

1 Relève également du secteur "Astronomie et Astrophysique » dans lequel la fiche de l'infrastructure est présentée.

2 Relève également du secteur «Astronomie et Astrophysique » dans lequel la fiche de l'infrastructure est présentée. 


\section{Organisation Européenne pour la Recherche Nucléaire - Grand Collisionneur Hadronique/European Organization for Nuclear Research - Large Hadron Collider}

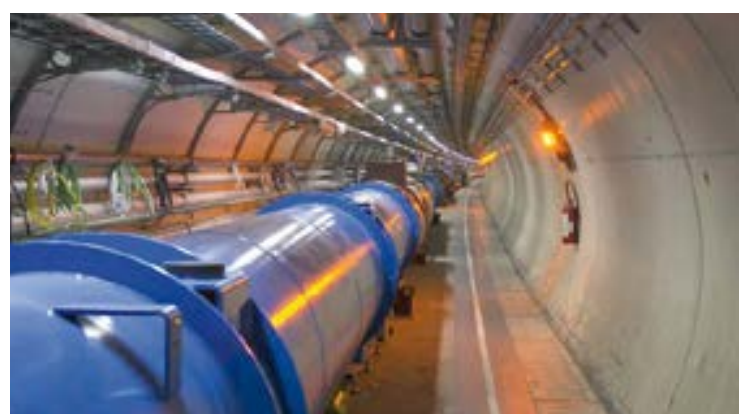

Type de l'infrastructure : OI

\section{Statut de l'infrastructure :}

Organisation Internationale

\section{Localisation de l'infrastructure :}

Genève

\section{Représentantes en France :}

Ursula BASSLER, Anne-Isabelle ETIENVRE

\section{Création :}

1954

\section{Exploitation :}

CERN : 1957

LHC : 2008

\section{Jouvence:}

LHC Phase I : 2019

LHC Phase II : 2027

Tutelles / Partenaires :

CEA, CNRS

\section{Contact en France :}

ursula.bassler@cnrs-dir.fr

anne-isabelle.etienvre@cea.fr

\section{www.lhc-france.fr}

\section{home.cern/fr}

Le CERN pilote la physique des particules en Europe et opère avec le LHC l'infrastructure mondiale la plus importante dans le domaine de la physique des particules. II poursuitégalement des programmes de recherche en physique nucléaire, en physique des neutrinos et de la R\&D en instrumentation et dans les techniques d'accélération. Pour les besoins de sa communauté il opère des infrastructures de calcul et s'apprête à jouer un rôle clé dans la structuration des infrastructures numériques en Europe.

Le LHC est actuellement le collisionneur de particules aux plus hautes énergies. II a atteint en 2015 son énergie nominale.

Avec la découverte du boson de Higgs, l'étude de ses propriétés ainsi que la recherche de la physique au-delà du modèle standard sont les premiers objectifs scientifiques. Une série d'upgrades du complexe d'accélérateurs va permettre d'augmenter l'intensité des faisceaux de particules pour atteindre une plus grande sensibilité à des interactions rares. La physique des interactions électrofaibles, celle des quarks b et c - et ainsi l'étude de la violation de symétrie CP, ainsi que les plasmas quark/gluons font partie des défis que le LHC permettra de relever dans les années à venir.

\section{IMPLICATIONSSOCIO-ÉCONOMIQUES}

\begin{abstract}
Les commandes passées auprès des entreprises françaises s'élèvent à $69,5 \mathrm{MFCH}$ pour les services et $78,6 \mathrm{MFCH}$ pour les fournitures ce qui correspond à des coefficients de retour respectifs de 2,86 et 1,57. EDF, ALSTOM, AIR LIQUIDE et THALES participent également aux études innovantes de pointe menées au CERN.
\end{abstract}

\section{DONNÉES}

Estimation du flux de données : 60 Po de données/an (80 Po/an en 2025).

Stockage : Les données sont distribuées sur une grille de calcul mondiale, dont $10 \%$ des capacités sont en France.

Accessibilité : Le CERN entreprend une politique d'accès ouvert des données, déjà en cours pour l'expérience CMS.

Présence dans des réseaux de données : WLCG (world-wide LHC Computing Grid) et LCG-France

\section{Coût de construction \\ LHC : 5,2 Md€ \\ Upgrade LHC : \\ Phase I: $20 \mathrm{M€}(\mathrm{FR})$, \\ Phase II : $50 \mathrm{M€}$ (FR, non-decidée) \\ Coût de fonctionnement : \\ CERN : $150 \mathrm{M} €(\mathrm{FR})$}

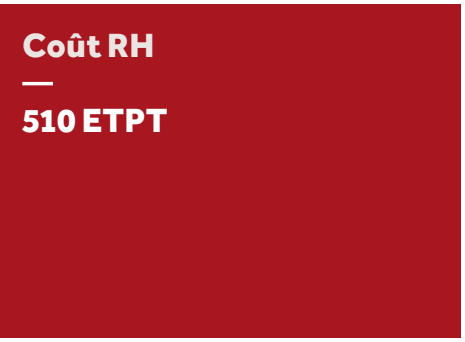

\section{Dimension internationale}

HL-LHC, ESFRI Landmark

Coordinateur : Fabiola Gianotti, Directrice Générale

Pays partenaires : DE, AT, BE, BU, DK, ES, FI, FR, EL, HU, IL, IT, NO, NL, PL, PT, SK, CZ, UK, SE, CH 


\section{EGO-VIRGO}

\section{Observatoire Européen Gravitationnel-VIRGO/ European Gravitational Observatory-VIRGO}

Le consortium franco-italien EGO, avec la collaboration Virgo, construit et gère l'interféromètre géant VIRGO destiné à observer des ondes gravitationnelles. Attendues lors d'événements sur les corps célestes, ces ondes courbent légèrement l'espace-temps, selon la théorie de la relativité générale. Le détecteur advanced Virgo est une version très améliorée de Virgo, dont les deux bras font $3 \mathrm{~km}$ de long, conçu pour mesurer les infimes variations de distance $\left(10^{-19} \mathrm{~m}\right)$ entre ses miroirs suspendus, induites par le passage d'une onde gravitationnelle. VIRGO travaille avec LIGO [Laser Interferometry Gravitational-Wave Observatory] aux USA qui a très récemment, réalisé les premières détections d'ondes gravitationnelles. LIGO et Virgo sont entrés en phase d'amélioration en 2010-2011. Concernant Advanced Virgo, ces améliorations portent sur le laser, les miroirs, la taille du faisceau, un système de compensation thermique..., et visent un gain d'un facteur 10 sur la sensibilité de l'antenne, soit un facteur 1000 sur le volume d'Univers observable. Ce détecteur de deuxième génération permettra la détection de plusieurs sources d'ondes gravitationnelles (Supernova, fusions de systèmes doubles de trous noirs et/ou étoiles à neutrons...) par an.

L'Allemagne et le Royaume-Uni ont construit l'antenne GEO600 de moindre sensibilité. Le Japon construit l'antenne souterraine KAGRA.

\section{IMPLICATIONSSOCIO-ÉCONOMIQUES}

La construction de l'interféromètre génère des innovations et des développements de R\&D dans le domaine des lasers (accords spécifiques avec Eolite puis ALS), des dépôts de couches minces, en métrologie...

\section{DONNÉES}

Estimation du flux de données : plus de 500 To/an de données brutes et 20 To de données réduites.

Stockage : EGO dispose de moyens de stockage et de calcul d'importance intermédiaire dédiés au stockage à court et moyen terme.

Accessibilité : les données de LIGO et Virgo sont enregistrées et regroupées dans une base de données commune gérée par LIGO.

\section{Coût de construction}

$151 M €+23,8 M €$

Advanced Virgo

dont 7 M€ de contribution

française

Coût de fonctionnement : $9 \mathrm{M} €$

dont 4,5 M€ de contribution

française

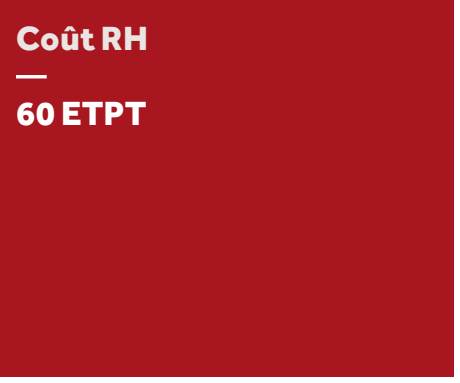

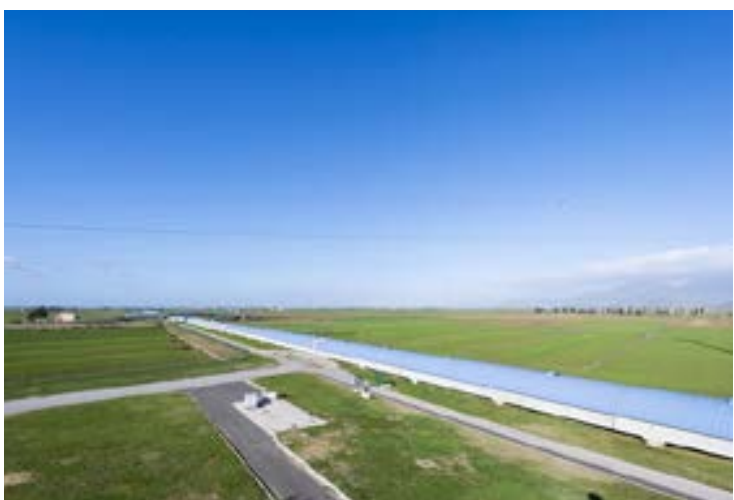

Type de l'infrastructure : TGIR

Statut de l'infrastructure :

Société civile de droit italien

\section{Localisation :}

Cascina, IT

\section{Représentant en France :}

Benoît MOURS

\begin{tabular}{|r|c|c|}
\hline Création: & Exploitation: & Maintenance : \\
\hline 1996 & 2003 & $2011-2016$ \\
\hline
\end{tabular}

\section{Tutelles / Partenaires :}

FR CNRS ( $50 \%$ ), IT INFN (50\%),

NL NIKHEF(in-kind)

\section{Contact en France :}

benoit.mours@lapp.in2p3.fr

www.ego-gw.it 


\section{Facility for Antiproton and Ion Research}

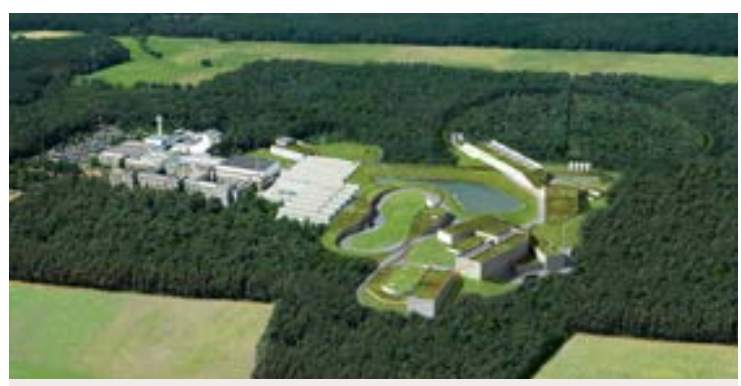

Type de l'infrastructure : TGIR

Statut de l'infrastructure :

$\mathrm{GmbH}$

\section{Localisation de l'infrastructure :}

Darmstadt, DE

\section{Représentants en France :}

Patricia ROUSSEL CHOMAZ, Fanny FARGET

\begin{tabular}{|r|c|} 
Création : & Exploitation : \\
\hline 2013 & 2022 \\
\hline
\end{tabular}

\section{Tutelles / Partenaires :}

FR (2,7\%), DE (69,89\%), Ru (17,65\%), IN (3,57\%),

PL (2,35\%), RO (1,18\%), SI (1,19\%), FI (0,5\%),

SE (0,99\%), UK associé à 0,5\%.

\section{Contact en France :}

patricia.roussel-chomaz@cea.fr

ffarget@admin.in2p3.fr

\section{www.fair-center.eu}

Le Projet FAIR a pour mission de construire et d'exploiter une nouvelle infrastructure de recherche européenne sur les antiprotons et les ions en Europe sur le site de GSI (société pour la recherche avec les ions lourds). Ce sera un ensemble d'accélérateurs basé sur la construction de deux synchrotrons supraconducteurs d'une circonférence de 1100 m, l'un sur l'autre dans le même tunnel. Ces synchrotrons fourniront différents faisceaux d'ions légers et lourds. Un dispositif adjacent d'anneaux de stockage. de cibles de production et de séparateurs fournira des faisceaux d'antiprotons et des faisceaux radioactifs secondaires.

Ces faisceaux de qualité et d'intensités inégalées sont dédiés principalement aux programmes de recherche en physique nucléaire (Interaction forte, structure des hadrons, plasmas, champs électromagnétiques très forts...) mais également dans de multiples domaines (science des matériaux et biologie). Les scientifiques sont regroupés en 4 grandes collaborations, pour construire les équipements qui seront utilisés sur FAIR: APPA : Atomic, Plasma Physics and Applications (biology, material science...), CBM : Compressed Baryonic Matter, NUSTAR : Nuclear Structure, Astrophysics and Reactions et PANDA : Anti-Proton Annihilation at Darmstadt. Cette infrastructure sera complémentaire de GANIL-SPIRAL2 et de ALICE et LHCb au CERN

\section{DONNÉES}

Estimation du flux de données : Plus de 30 Po/an.

Accessibilité : Les données seront réutilisables par d'autres scientifiques sous réserve de l'accord de la collaboration qui a obtenu les données.

Présence dans des réseaux de données : Le centre TierO constituera le noyau du réseau HPC de FAIR, avec une liaison $1 \mathrm{~Tb} / \mathrm{s}$ avec les systèmes HPC des universités voisines. Les systèmes distants seront servis par les technologies de grille et de cloud.

\section{Coût de construction \\ 1,357 M€ dont 2,7\% de participation française \\ Coût de fonctionnement: \\ Estimé à $240 \mathrm{M€dont}$ \\ $2 \%$ de contribution française}

\section{Coût RH}

$\overline{60}$ ETPT 


\section{GANIL-SPIRAL2}

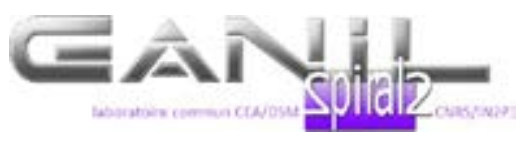

\section{Grand Accélérateur National d'lons Lourds - Système de production d'lons Radioactifs en Ligne de $2^{e}$ génération}

Le GANIL, outil de recherche pluridisciplinaire au service de la communauté nationale et internationale, est un laboratoire de recherche fondamentale et appliquée en physique atomique, physique nucléaire et physique de la matière condensée. Le GANIL offre un large éventail de faisceaux d'ions accélérés, au moyen de 3 cyclotrons en cascade, du carbone à l'uranium, permettant en particulier la création et l'accélération de noyaux exotiques.

Le GANIL est I'un des 5 grands laboratoires au monde pour la recherche avec des faisceaux d'ions (GSI-Allemagne, RIBF/RIKEN-Japon, MSU/ NSCL-USA et FLNR/JINR Dubna-Russie). C'est un outil de pointe dans différents domaines, notamment la physique nucléaire fondamentale et astrophysique. En effet, GANIL permet de produire et d'étudier les noyaux qui n'existent pas sur Terre : les noyaux exotiques.

SPIRAL 2 est une nouvelle infrastructure composée d'un accélérateur linéaire d'ions légers et lourds et de 3 nouvelles salles : NFS : Neutrons For Science qui délivrera un faisceau de neutrons d'énergie jusqu'à $40 \mathrm{MeV}$, S3: Super Séparateur Spectromètre conçu pour exploiter les faisceaux stables de très hautes énergies et DESIR: Salle d'expérience avec les noyaux exotiques à basse énergie, qui recevra les faisceaux de Spiral 1 et de S3 et à plus long terme, d'un bâtiment de production des faisceaux radioactifs.

\section{IMPLICATIONSSOCIO-ÉCONOMIQUES}

Le GANIL est un élément structurant avec :

- création sur son campus des laboratoires CIMAP, CYCERON, CURBE,

l'entreprise CYCLOPHARMA et d'un centre de l'hadronthérapie ARCHADE:

- la création de 30 start-ups via Normandie Incubation;

1 transfert technologique (entreprise Pantechnik spécialisée dans

les sources d'ions). 1 à 2 brevets/an sont déposés;

la participation à Nucléopolis.

\section{DONNÉES}

Estimation du flux de données : 5 à 10 To de données/an avec prévision de 200 To à 800 To vers 2017

Stockage : Les données sont stockées sur un serveur local au GANIL d'une capacité de 200 To.

Accessibilité : Les données sont accessibles pendant les expériences au GANIL puis durant 3 à 6 mois en fonction des expériences.

\section{Coût de construction}

GANIL : 911 MF (1999) + SPIRAL2 phase 1+ : 128 M€

Coût de fonctionnement : $14 \mathrm{M€}$

\section{Dimension internationale}

SPIRAL2 a été inscrit comme projet dans la roadmap ESFRI en 2006. Il est devenu « Landmark » en 2016.

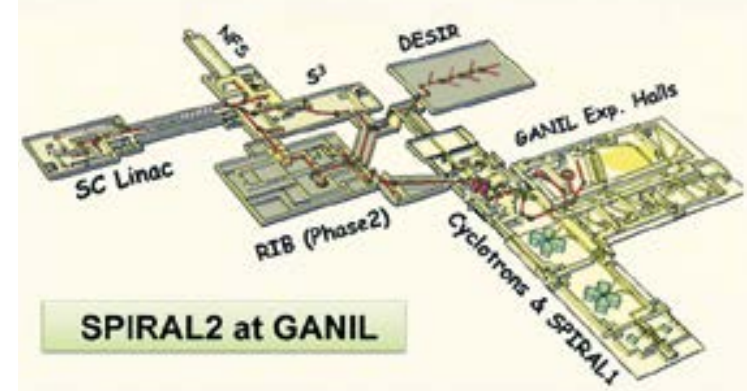

Type de l'infrastructure : TGIR

Statut de l'infrastructure:

Groupement d'Intérêt Economique

\section{Localisation :}

Caen

Responsable de l'infrastructure:

Florent STALEY

\section{Création: Exploitation:}

$1976 \quad 1983$

\section{Jouvence:}

2010-2016 : Spiral2 Phase 1+

Tutelles / Partenaires :

CEA, CNRS

\section{Contact en France :}

accueil@ganil.fr

www.ganil-spiral2.eu 


\section{Kilometre Cube Neutrino Telescope}

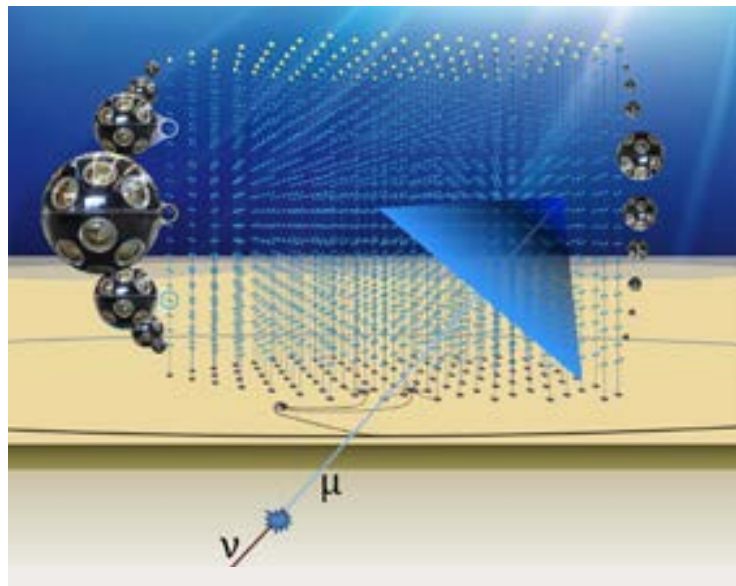

Type de l'infrastructure : IR

\section{Localisation de l'infrastructure:}

Toulon, Capo Passero en Sicile

\section{Représentant en France :}

Paschal COYLE

\section{Création :}

2014

\section{Exploitation :}

2017 : 7 lignes en France, 24 lignes en Italie

\section{Jouvence:}

2017-2020: KM3NeT 2.0 upgrade des sites : France (115 lignes), Italie (230 lignes)

\section{Tutelles / Partenaires :}

CNRS, AMU, Univ. Paris 7, Univ. Strasbourg, Univ. Clermont-ferrant, Univ. Lyon, UTLN, OCA, Univ. Paris Sud

\section{Pays partenaires :}

CY, ES, FR, DE, EL, IE, IT, MA, NL, PL, RO, UK

\section{Contact en France :}

coyle@cppm.in2p3.fr

\section{www.km3net.org}

KM3NeT est un projet européen d'observatoire de neutrinos qui doit être installé en mer Méditerranée. Cette installation est conçue pour détecter la très faible lumière générée par les neutrinos ayant traversé la Terre. Deux sites sont en cours de construction au large de Toulon en France (Oscillation Research with Cosmics in the Abyss-ORCA) et en Sicile (Astroparticles Research with Cosmics in the Abyss-ARCA) optimisés pour la détection de neutrinos respectivement atmosphériques de basse énergie ( $3 \mathrm{GeV}-100$ $\mathrm{GeV}$ ) et cosmiques de grande énergie ( $1 \mathrm{TeV}-10 \mathrm{PeV}$ ).

ORCA situé à $2500 \mathrm{~m}$ de profondeur sera constitué de 115 lignes flexibles ancrées sur le fond marin, espacées de $20 \mathrm{~m}$, portant des détecteurs (Digital Optical Modules DOM) distants de $9 \mathrm{~m}$. Pour ARCA situé à $3500 \mathrm{~m}$ de profondeur, les espacements sont beaucoup plus larges : $90 \mathrm{~m}$ entre les lignes et $36 \mathrm{~m}$ entre les DOM. Ce télescope prendra la suite de l'expérience ANTARES avec une sensibilité améliorée d'un ordre de grandeur.

Un des objectifs majeurs de KM3NeT est la mesure de la hiérarchie de masse des neutrinos ainsi que la découverte et l'étude des sources astrophysiques de neutrinos cosmiques. Ces infrastructures permanentes en mer profonde offrent d'importantes opportunités de synergie avec les communautés des Sciences de la Terre, de la Mer et de l'Environnement. Au niveau mondial, il n'existe pas d'autre infrastructure de ce type.

\section{IMPLICATIONSSOCIO-ÉCONOMIQUES}

Les retombées sont diverses

nombreux partenariats industriels : Alcatel, AIM, Comex, CREDAM, Cybernetix, Degreane, ECA, Euroceanique, Foselev marine, iXSurvey, iXSea, Genisea, Osean, Orange Marine, etc. ;

création d'une start-up PowerSea développant des systèmes de connexions innovants dans le domaine de la connectique marine; 1 brevet déposé.

\section{DONNÉES}

Estimation du flux de données : 8 To/an avec 31 lignes et 106 To/an pour 115 lignes.

Stockage : Stockage au Centre de Calcul de Lyon (CNRS-IN2P3)

Accessibilité : Données publiques après un délai de deux ans. Les données environnementales immédiatement accessibles.

Présence dans des réseaux de données : Plateforme Mistrals SEDOO (mistrals.sedoo.fr/MEUST/)

\section{Coût de construction \\ 一 \\ Phase 1 : Infrastructures + \\ 31 lignes = $31 \mathrm{M€}$ \\ Phase 2.0 : 345 L. 120 M€ \\ Phase 3:660 L.220M€ \\ Coût de fonctionnement : \\ $1 \mathrm{M} € /$ an (FR : 0,4 M€, IT : 0,6 M€)}

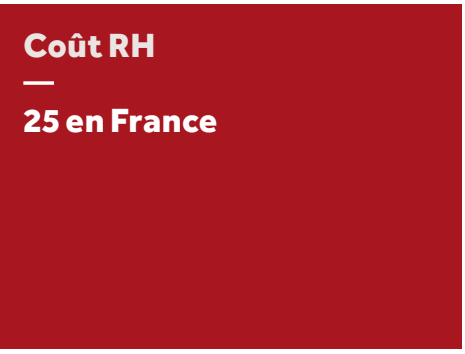

\section{Dimension internationale}

KM3NeT: ESFRI Roadmap

Coordinateur : Maarten de Jong

EMSO : European Multidisciplinary Seafloor and Water Column Observatory 


\section{Large Synoptic Survey Telescope}

Le Large Synoptic Survey Telescope (LSST) est un grand télescope en construction au Chili depuis 2014. II commencera son program d'observation en 2022. II a une conception compacte basée sur un ensemble de 3 miroirs (8,4 m, $5 \mathrm{~m}$ et 3,4 m), aboutissant a une étendue très importante de $319 \mathrm{~m}^{2} \mathrm{deg}^{2}$ : chaque vue panoramique de LSST avec sa caméra de 3200 millions de pixels, couvrira une surface équivalente à 40 fois la pleine lune. Sa vitesse d'exécution combinée à la profondeur et à la largeur de son champ de vue, donnent à LSST des capacités d'observation uniques.

Pendant ses 10 ans de relevés, LSST cartographiera l'ensemble du ciel visible deux fois par semaine, fournissant un film précis de «l'univers dynamique». Ces images seront analysées en temps réel afin d'identifier les objets qui ont changé ou se sont déplacés: des explosions de supernova à l'autre bout de l'univers aux astéroïdes pouvant impacter la terre. Les objectifs scientifiques principaux de LSST sont d'étudier la nature de la matière noire et d'améliorer notre compréhension de ce qu'est l'énergie noire. Au delà de ces deux sujets et de l'étude de l'univers variable, les chercheurs pourront, à l'aide du volume gigantesque de données du LSST, étudier l'ensemble des différentes structures présentes dans l'univers y compris notre système solaire et la voie lactée.

Sans compétiteur au sol, LSST est complémentaire des programmes spatiaux destinés à l'étude de l'énergie noire tel le satellite Euclide (Projet ESA).

\section{IMPLICATIONS SOCIO-ÉCONOMIQUES}

Le défi scientifique et technologique que représentent la réalisation et la construction de ce télescope entrainera en France des innovations dans les capteurs $C C D$ et leur électronique de lecture, dans la mesure fine de grande optique ainsi que dans les bases de données et le traitement de données dans le cadre «Big Data » correspondant à LSST.

\section{DONNÉES}

Estimation du flux de données : 15 To de données/nuit

Stockage : stockage au centre de calcul de I'IN2P3 (CC-IN2P3)

Accessibilité : Accessibles par les chercheurs à travers des serveurs de données et dans certains cas, par le grand public.

\section{Coût de construction}

\section{-}

$625 \mathrm{M} €$ (US) + contribution française camera 14,4 M€

Coût de fonctionnement :

$0,1 \mathrm{M} € /$ an de contribution

française pendant la construction

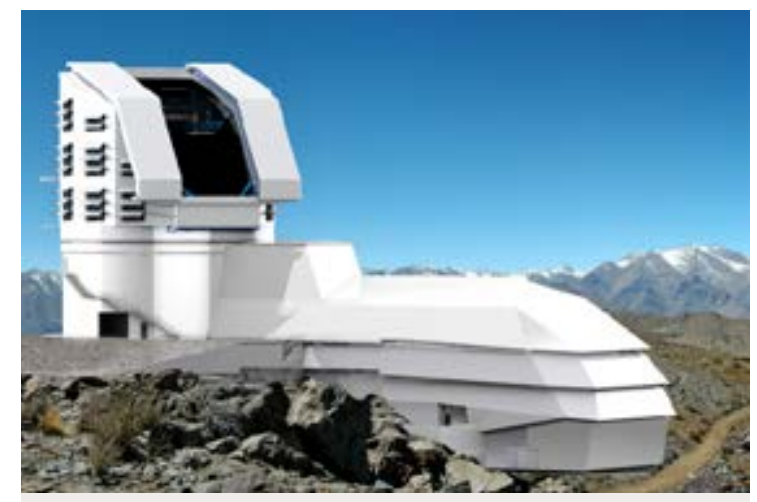

Type de l'infrastructure : IR

\section{Statut de l'infrastructure :}

Société américaine

Localisation:

Cerro Pachon, Chili

Représentant en France :

Pierre ANTILOGUS

\begin{tabular}{|r|c|}
\hline Création : & Exploitation : \\
\hline 2014 & 2022 \\
\hline
\end{tabular}

\section{Tutelles / Partenaires:}

USA, FR (CNRS), Chili; Pays qui participeront à l'exploitation: USA, FR, CL, AU, BR, CN, HU, IN, RS, ZA, UK

\section{Contact en France : \\ p.antilogus@in2p3.fr}

\section{Isst.in2p3.fr}

\section{Isst.org}




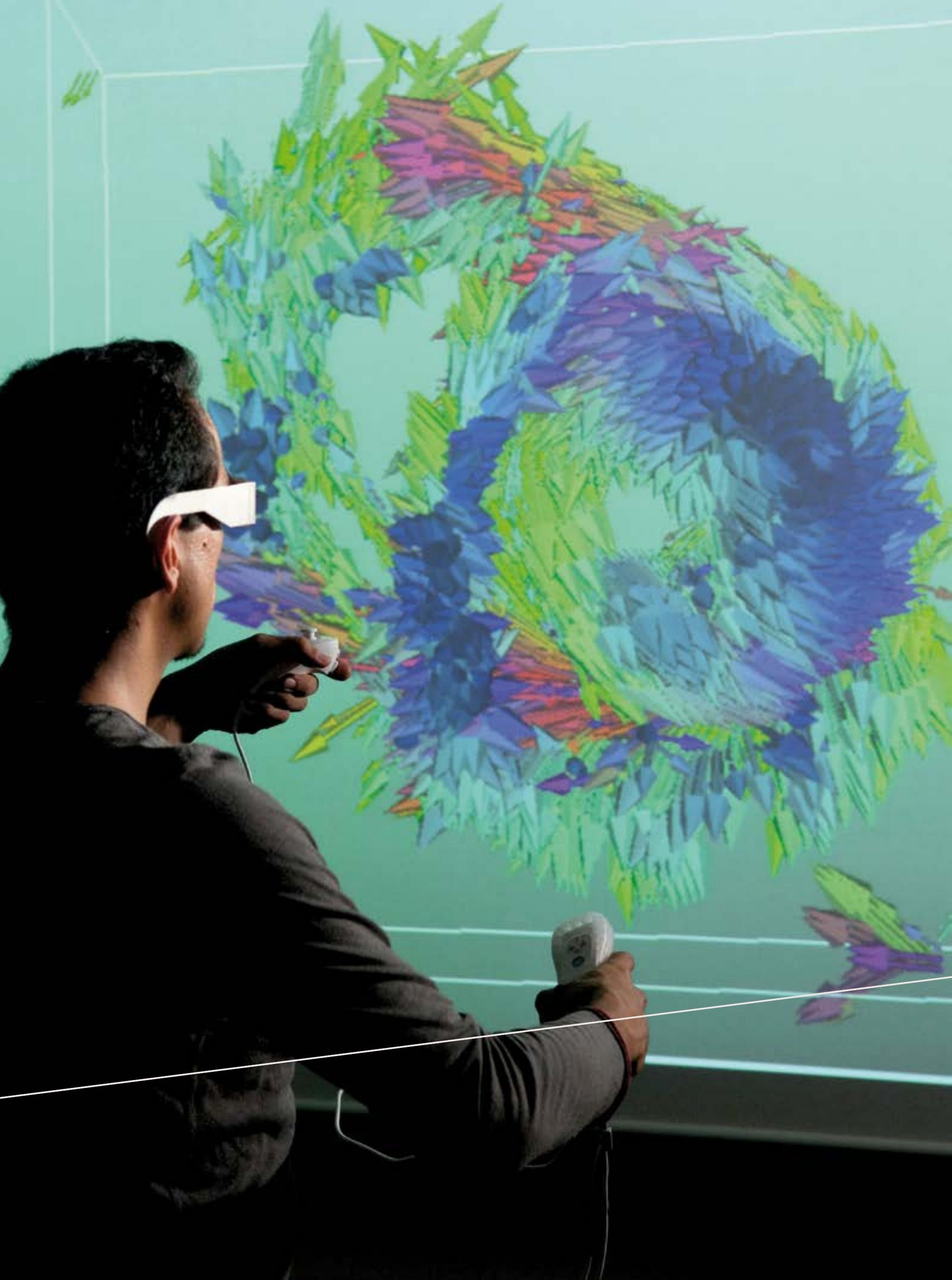




\section{SCIENCESET \\ TECHNOLOGIES DU NUMÉRIQUE; MATHÉMATIQUES}




\section{Sciences et technologies}

\section{du numérique; Mathématiques}

Le numérique évolue à une vitesse considérable, avec de nombreux nouveaux dispositifs technologiques (réalité virtuelle, objets communicants, capteurs), qui induisent une progression exponentielle de ses capacités et de son ubiquité. En particulier, pour ce qui concerne les grandes masses de données, leur importance pour les sciences de demain va croître, avec des sources de données, centralisées (grands instruments en physique, observatoires), ou distribuées (séquenceurs à haut débit, réseaux de capteurs). La France se dote d'une stratégie pour répondre à une demande forte en matière de stockage, de traitement et de transmission des données.

Les infrastructures numériques sont, pour la plupart, des plateformes organisées en réseau. Ces infrastructures, de développement récent, ont l'ambition de coordonner un ensemble d'actions menées sur le territoire national en lien, le plus souvent, avec l'espace européen.

Plus précisément, les infrastructures numériques, dans la feuille de route actuelle, consistent en quatre infrastructures de calcul (GENCI, CCIN2P3, Grid'5000 et France Grilles) et deux infrastructures de réseaux (RENATER et FIT). Plusieurs sont intégrées à des infrastructures européennes ou internationales : GENCI (composante française de PRACE), RENATER (composante française de GÉANT), France Grilles (composante française de EGI), FIT (qui s'inscrit dans la plateforme européenne OneLab), et le CC-IN2P3 (intégré au réseau international Worldwide LHC Computing Grid).

D'un point de vue fonctionnel, ces infrastructures peuvent être classées en deux familles :

- d'une part, les infrastructures numériques de production, qui sont au service de toutes les communautés scientifiques (GENCI/TGCC, GENCI/IDRIS, GENCI/CINES, GENCI/PRACE, RENATER, France Grilles, CCIN2P3);

- d'autre part, les infrastructures dédiées aux recherches en mathématiques (GERM) et aux expérimentations en sciences du numérique (Grid5000, FIT, RNRVA).

Deux très grandes infrastructures (TGIR) constituent la colonne vertébrale des infrastructures numériques: GENCl et RENATER.
L'infrastructure de calcul GENCI apporte une offre de calcul à haute performance (supercalculateurs), basée sur trois grands centres nationaux du CNRS, du CEA et des Universités : I'IDRIS, le TGCC et le CINES. En complément, le CC-IN2P3 et France Grilles apportent une offre de calcul distribué et d'hébergement de grands volumes de données. De plus, GENCl est liée à des plateformes intermédiaires d'hébergement et de traitement de données.

L'infrastructure de réseau RENATER apporte une offre de communication numérique, sécurisée à haut débit, à l'ensemble des établissements et des communautés de l'Enseignement Supérieur et de la Recherche, ainsi que l'interconnexion aux réseaux mondiaux. Elle a aussi joué un rôle moteur dans le développement et la proposition de services informatiques, tels que la visioconférence.

Par ailleurs, Grid'5000 etFIT sont des infrastructures d'expérimentation pour les recherches en sciences du numérique, ce qui exclut des services opérationnels. GRID'5000 est un instrument scientifique flexible et de grande taille pour le support de la démarche expérimentale, dans les domaines de la recherche en informatique, portant en particulier sur les systèmes parallèles et distribués, ainsi que les Clouds. FIT est une plate-forme d'expérimentation, qui permet l'accès distant à des ressources informatiques variées dans le contexte de l'Internet des Objets, pour réaliser des tests de performance, d'interopérabilité et de sécurité.

À ces infrastructures s'ajoutent les projets GERM (Grand Equipement pour la Recherche en Mathématiques), et RNRVA (Réseau National de plates-formes de Réalité Virtuelle et Augmentée). GERM est le prolongement du labex CARMIN, qui regroupe les 4 centres d'accueil mathématiques, CIMPA, CIRM, IHES et IHP. Ce projet a vocation à conforter la suprématie française en recherche fondamentale et appliquée pour les mathématiques, avec le développement de structures d'accueil et d'échanges de renommée mondiale. RNRVA est un réseau national dédié aux infrastructures haut-niveau pour la réalité virtuelle et augmentée, porté par l'AFRV (Association Française de Réalité Virtuelle). L'objectif, à travers des connections haut-débits, des équipements spécifiques et des personnels dédiés, est de traiter de grands défis scientifiques relatifs à des besoins industriels. 


\section{戈 LISTE DES INFRASTRUCTURES DE RECHERCHE}

羊 DANS LE DOMAINE SCIENCES ET TECHNOLOGIES DU NUMÉRIQUE; MATHÉMATIQUES

\begin{tabular}{|c|c|c|c|}
\hline CATÉGORIE & NOM & NOM COMPLET & ESFRI \\
\hline TGIR & GENCl & Grand Équipement National de Calcul Intensif & \\
\hline TGIR & RENATER & $\begin{array}{l}\text { RÉseau National de télécommunications pour } \\
\text { la Technologie, I'Enseignement et la Recherche }\end{array}$ & \\
\hline IR & CCIN2P3 & Centre de Calcul de l'IN2P3/CNRS & \\
\hline IR & FIT & Futur Internet des Objets & \\
\hline IR & France Grilles & France Grilles & \\
\hline IR & Grid 5000 & Grid'5000 & \\
\hline Projet & GERM & $\begin{array}{l}\text { Grand Équipement pour la Recherche } \\
\text { en Mathématiques }\end{array}$ & \\
\hline Projet & RNRVA & $\begin{array}{l}\text { Réseau National de plates-formes de Réalité } \\
\text { Virtuelle et Augmentée }\end{array}$ & \\
\hline
\end{tabular}




\section{Grand Équipement National de Calcul Intensif}

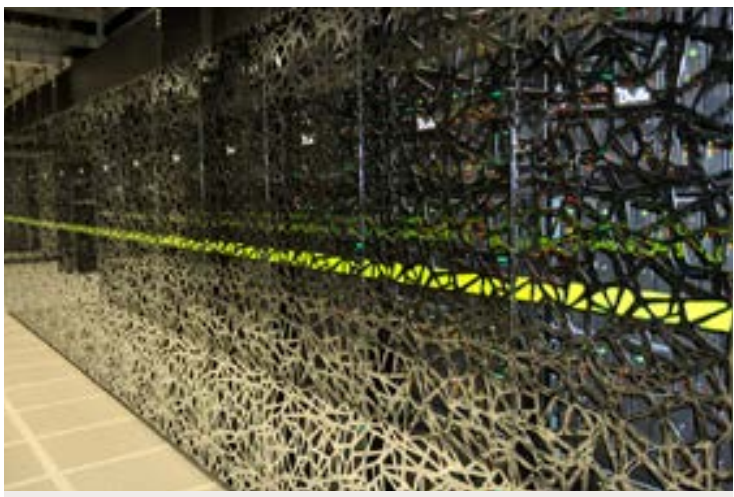

Type de l'infrastructure : TGIR

Statut de l'infrastructure :

Société Civile

Localisation de l'infrastructure :

Paris

Localisation des autres sites :

Bruyères-le-Châtel, Orsay, Montpellier

Responsable de l'infrastructure :

Catherine RIVIÈRE

\section{Création :}

2007

Tutelles / Partenaires :

MENESR, CEA, CNRS, CPU, Inria

\section{Contact en France :}

contact@genci.fr

www.genci.fr
GENCI met à disposition de très gros calculateurs à destination des scientifiques français afin qu'ils réalisent des travaux de pointe nécessitant I'utilisation de la simulation numérique. GENCl a trois missions principales: - porter la stratégie nationale d'équipement en moyens de calcul intensif au bénéfice de la communauté scientifique nationale. GENCI assure la maîtrise d'ouvrage des moyens de calcul nationaux, répartis dans trois centres de calcul : le TGCC du CEA à Bruyères-le-Châtel, I'IDRIS du CNRS à Orsay et le CINES à Montpellier. À ce titre, GENCl a la responsabilité du plan d'investissement pluriannuel d'évolution des supercalculateurs installés dans ces trois centres;

- promouvoir l'utilisation de la simulation numérique et du calcul intensif. GENCI mène ou participe à différents projets de diffusion de la simulation numérique, dont l'Equipex Equip@meso et I'Initiative HPC-PME;

- participer à la réalisation d'un écosystème intégré du calcul intensifà l'échelle européenne. GENCl représente la France dans l'infrastructure européenne PRACE (Partnership for Advanced Computing in Europe). GENCl est un des 4 membres hébergeurs de PRACE (aux côtés de GCS pour l'Allemagne, CINECA pour l'Italie et BSC pour l'Espagne) qui met à disposition des supercalculateurs de classe mondiale pour les chercheurs européens.

\section{IMPLICATIONSSOCIO-ÉCONOMIQUES}

Les industriels français sont éligibles gratuitement aux moyens de calcul de GENCI. Ce dispositif particulier a déjà été utilisé par ArcelorMittal, Veolia Environnement, EDF et Continental.

Depuis 2010, GENCl porte, avec Bpifrance et Inria, I'Initiative HPC-PME pour aider les PME françaises à utiliser la simulation numérique. Des PME comme HydrOcean ou Algotech ont déjà bénéficié de ce programme.

\section{DONNÉES}

Estimation du flux de données : Les données produites par les supercalculateurs sont stockées dans les centres de calcul. Elles sont ensuite rapatriées par les utilisateurs.

Stockage : 35 Po répartis dans les centres de calcul Accessibilité : Via RENATER

\section{Coût de fonctionnement}

$1,8 \mathrm{M} €$
Coût RH

\section{Dimension internationale}

GENCI représente la France au sein de l'infrastructure européenne de recherche PRACE (Partnership for Advanced Computing in Europe) inscrite sur la feuille de route ESFRI en 2006 et ESFRI Landmark. PRACE est une Association Internationale Sans But Lucratif(AISBL) de droit belge, créée en 2010 et rassemblant 25 partenaires et qui a pour mission la mise à disposition de ressources de classe mondiale en termes de calcul intensif et de gestion des données pour les chercheurs académiques et industriels européens.

Coordinateur : Sanzio Bassini (Président du Conseil)

Pays partenaires : 25 pays sont membres de PRACE : AT, BE, BG, CY, CZ, DK, FI, FR, DE, EL, HU, IE, IT, NL, NO, PL, PT, SK, ES, SE, CH, TR, UK. Seuls 4 de ces pays (DE, FR, IT, ES) hébergent un supercalculateur.

Site internet : $\underline{\text { www.prace-ri.eu }}$ 


\section{RÉseau National de télécommunications pour la Technologie, l'Enseignement et la Recherche}

En soutien à l'ensemble de la communauté scientifique, technologique et d'enseignement, RENATER met en œuvre un backbone national de communication (13000 km de fibres optiques noires), des équipements de génération des signaux, de commutation, de super et hyper vision. Les missions de RENATER sont:

- de fournir aux acteurs de la communauté recherche et éducation les moyens de communication numérique haut débit et de gestion des données liées en France (métropolitaine, dans les ROM et dans les COM) sur la base de réseaux, d'infrastructures et de services;

- d'assurer que l'ensemble de ces moyens sont sécurisés:

- d'assurer l'interconnexion aux réseaux de recherche etéducation mondiaux;

- d'assurer les travaux des équipes en réseau et de répondre aux besoins avancés et innovants de la communauté recherche et éducation ;

- d'assurer une mission de conseil, d'expertise, de fournir des moyens ou des services de communication dans ses domaines de compétence auprès de l'État et d'autres entités publiques français ou étrangers, dans la mesure où cela n'impose pas au Groupement des obligations incompatibles avec sa mission de fourniture de services à la communauté recherche et éducation.

\section{IMPLICATIONSSOCIO-ÉCONOMIQUES}

Tous les secteurs de l'innovation travaillant de manière collaborative sont amenés à utiliser l'infrastructure RENATER.

RENATER sert également de dorsale au RIE (Réseau Interministériel de l'État) en fournissant les liaisons optiques de ce réseau.

\section{Coût de construction}

$30 \mathrm{M} €$

Coût de fonctionnement : $22 \mathrm{M€}$

\section{Coût RH $\overline{70} \mathrm{ETP}$}
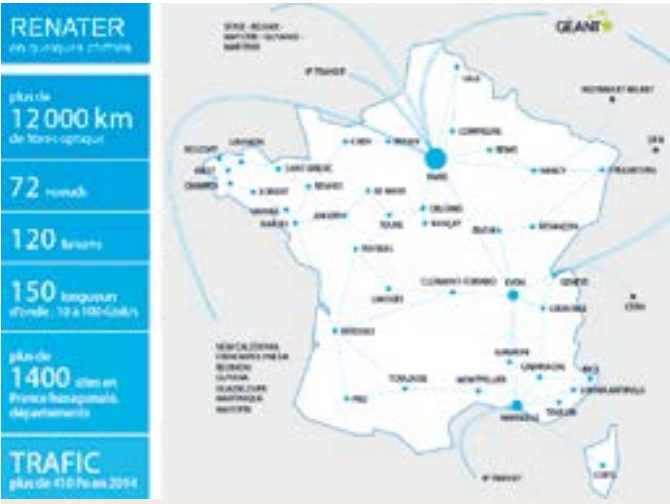

Type de l'infrastructure : TGIR

Statut de l'infrastructure:

Groupement d'Intérêt Public

\section{Localisation :}

Paris

\section{Localisation des autres sites :}

IR distribuée

Responsable de l'infrastructure:

Patrick DONATH

\section{Création :}

1993

\section{Dimension internationale}

RENATER est connecté au réseau pan-européen GEANT par l'hébergement de 3 de ses nœuds (Paris, Marseille, Genève).

Coordinateur : GEANT Association (association de droit néerlandais) dont le siège est situé à Amsterdam (NL) et les bureaux à Amsterdam (NL) et Cambridge (UK)

Pays partenaires : 43 Réseaux Nationaux de Recherche et d'Education (NREN) de pays européens ou ayant un accord de collaboration avec le programme européen pour la recherche et le développement.

RENATER a également établi des accords avec des pays africains pour l'aide à la mise en place de réseaux dans ces pays.

Site internet : www.geant.net
Contact en France :

support@renater.fr

www.renater.fr

\section{Tutelles / Partenaires :}

MENESR, CNRS, CPU, CEA, Inra, CNES, Inria, Inserm, ONERA, Cirad, Irstea, IRD, BRGM 


\section{CC-IN2P3}

\section{Centre de Calcul de I'IN2P3 / CNRS}

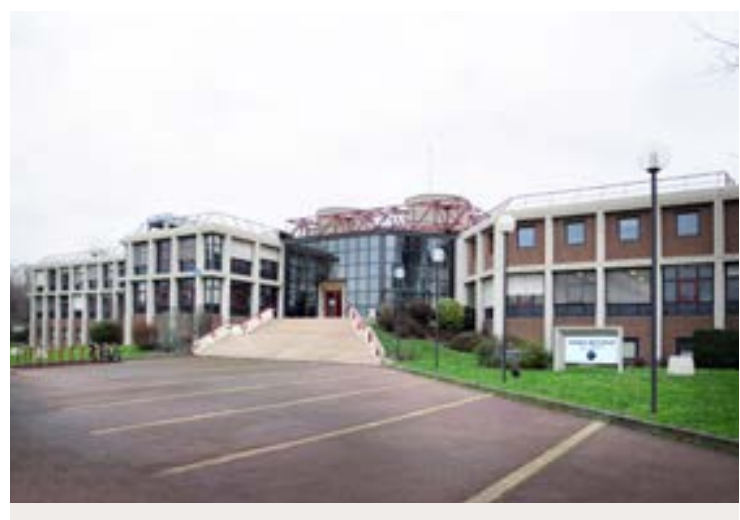

Type de l'infrastructure : IR

\section{Statut de l'infrastructure :}

Unité de services et de recherche

\section{Localisation de l'infrastructure : \\ Villeurbanne}

\section{Responsable de l'infrastructure :}

Pierre-Etienne MACCHI

\section{Création :}

1986

\section{Tutelles / Partenaires : \\ CNRS}

\section{Contact en France :}

accueil@cc.in2p3.fr

\section{cc.in2p3.fr}

Le CC-IN2P3 a développé une expertise technique de pointe dans les domaines suivants:

- calcul et traitement de données;

- stockage massif de données;

- bases de données relationnelles, réseaux, administration système;

- support aux utilisateurs;

- systèmes d'information.

En outre, il possède une forte expertise dans les technologies de pointe que sont les grilles de calcul ou le cloud computing.

Il est ainsi l'un des 14 datacenter internationaux déployés dans le cadre du projet Worldwide LHC Computing Grid (W-LCG). II fournit $11 \%$ des ressources mondiales nécessaires à la simulation, au traitement et à l'analyse des données des quatre expériences LHC. Le CC-IN2P3 est l'un des acteurs majeurs du projet LCG-France en fournissant les moyens humains et techniques nécessaires et l'épine dorsale de la grille de calcul française.

Le CC-IN2P3 est très impliqué dans plusieurs projets d'astroparticules et d'astrophysique. En astrophysique spatiale, le CC-IN2P3 est I'un des centres informatiques majeurs pour les missions Planck/HFI, Fermi et AMS sur la station spatiale internationale.

Conjointement avec le centre de calcul américain de NCSA, il assurera à parts égales le traitement des données du projet LSST et mettra à disposition des chercheurs l'intégralité des données issues de ce télescope. Dans le cadre de la mission spatiale EUCLID il sera le principal élément du segment sol français en fournissant 30\% des ressources nécessaires à la production de la première data release.

\section{DONNÉES}

\section{Estimation du flux de données : 50 Po par an}

Stockage : 18 Po sur disques à performance standard, 2 Po sur disque à haute performance, 31 Po de données stockées sur bande (capacité de stockage théorique de 340 Po), 1,1 Po de données sauvegardées (Capacité de stockage théorique de 5,5 Po)

Accessibilité : Via RENATER

Coût de fonctionnement
7,1 M€

\section{Coût RH}

78,6 ETPT 


\section{Futur Internet des Objets}

L'évolution vers un monde de plus en plus numérique requiert des outils de test et d'expérimentation qui permettent d'aider à la conception et à la validation des solutions proposées. La cible concerne des technologies de cœur tels que le cloud, l'internet des objets ou les réseaux d'infrastructures mais aussi les "verticaux» tels que la santé, les transports, l'énergie qui se nourrissent de technologies de capture de données pour alimenter leurs chaînes de connaissance. FIT propose un vaste choix de technologies (Internet des objets, réseaux radio sans-fils, overlays, SDN et Cloud) mais surtout une interface unique d'accès et de nombreux outils de configuration et de mesure. FIT permet d'aborder un vaste ensemble de questions grâce à son approche de fédération et d'assemblage de ressources informatiques et de communication de base. L'objectifest d'assister la conception avant le déploiement effectif et d'en diminuer le coût et le temps nécessaires tout en améliorant la robustesse des systèmes.

La mission de FIT est de fournir une plateforme expérimentale au travers de la fédération d'infrastructures, compétitive au niveau mondial, permettant d'incuber des expériences avancées et de stimuler une large base d'utilisateurs provenant du monde de la recherche et de l'industrie.

\section{IMPLICATIONSSOCIO-ÉCONOMIQUES}

Les industriels Alcatel-Lucent, Orange, et Thales sont membres du Comité de pilotage.

FIT collabore avec Institute for Information Industry (III) à Taiwan avec le déploiement d'un testbed loT-Lab.

FIT développe un modèle d'adhésion pour entreprises, basé sur les modèles PlanetLab Europe et OneLab.

\section{DONNÉES}

Stockage : Projet de service de stockage permettant aux utilisateurs de sauvegarder les données générées.

Accessibilité : FIT souhaite mettre en œuvre une politique de données ouvertes. Néanmoins, les données issues du monde industriel ou présentant un caractère de confidentialité (santé, vie privée, etc.) seront protégées.

Présence dans des réseaux de données : FIT fait partie de la fédération OneLab.

\section{Coût de construction}

$12 \mathrm{M€}$

Coût de fonctionnement : $1,5 \mathrm{M€}$

\section{Coût RH}

11 ETP

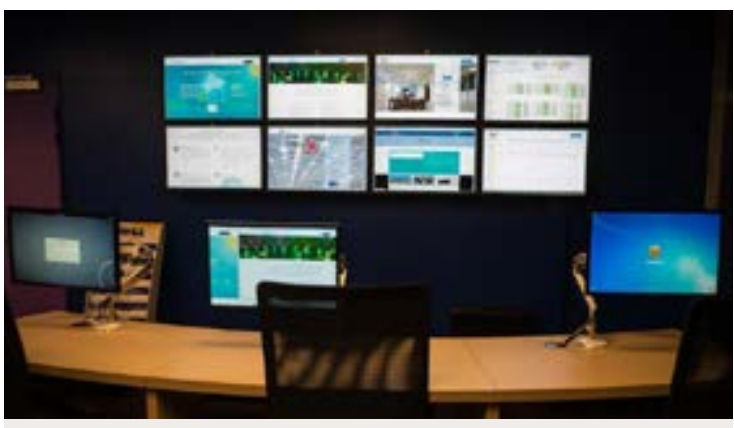

Type de l'infrastructure : IR

\section{Localisation :}

Paris

\section{Localisation des autres sites :}

Évry, Grenoble, Lille, Lyon, Rocquencourt, Sophia-Antipolis, Strasbourg

\section{Responsable de l'infrastructure :}

Serge FDIDA

\section{Création: Exploitation: \\ 2011 \\ 2014}

\section{Tutelles / Partenaires :}

UPMC, CNRS, Inria, IMT, UNISTRA

\section{Contact en France :}

serge.fdida@upmc.fr

\section{fit-equipex.fr}

\section{Dimension internationale \\ Fédération OneLab \\ Coordinateur : Serge Fdida \\ Pays partenaires : $\mathrm{FR}, \mathrm{BE}, \mathrm{DE}, \mathrm{EL}$ \\ Site internet : onelab.eu}




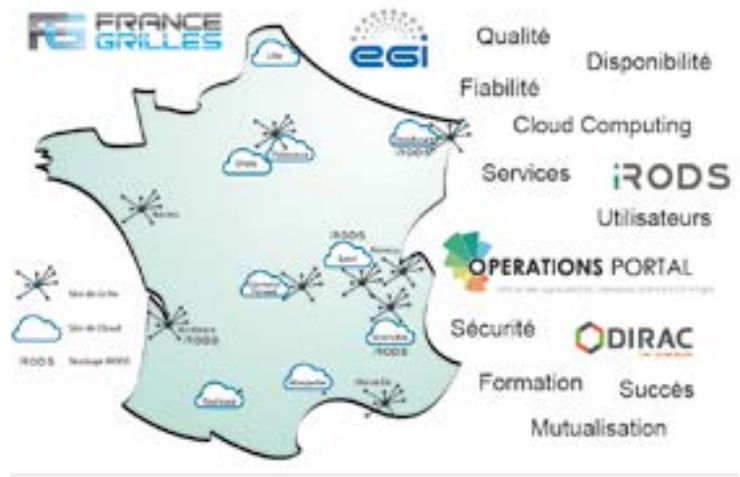

Type de l'infrastructure : IR

\section{Statut de l'infrastructure :}

Groupement d'Intérêt Scientifique

Localisation de l'infrastructure :

Aubière

\section{Localisation des autres sites :}

IR distribuée

Responsable de l'infrastructure : Vincent BRETON

\section{Création :}

2010

Tutelles / Partenaires :

CEA, CNRS, CPU, Inra, Inria, Inserm, RENATER

\section{Contact en France :}

info@france-grilles.fr

\section{www.france-grilles.fr}

L'infrastructure informatique nationale distribuée de France Grilles est pluridisciplinaire, ouverte à toutes les disciplines, ainsi qu'aux pays en développement. L'objectif est de faire de cette infrastructure commune un espace d'échanges et de collaborations au sein et au travers des disciplines et des institutions. Les missions principales de France Grilles sont les suivantes:

- établir et opérer une infrastructure nationale de grille de production, pour le traitement et le stockage de données scientifiques massives;

- contribuer avec les autres états membres impliqués au fonctionnement de l'infrastructure européenne EGI (www.egi.eu) et définir les modalités de la participation française à l'association EGI.eu qui coordonne la fédération EGI;

- favoriser les rapprochements et les échanges entre les équipes travaillant sur les grilles de production et les grilles de recherche.

Ces missions s'étendent depuis 2012 au domaine du Cloud Computing. En France, les grilles de production rassemblent plus de 40000 processeurs et environ 25 Po de stockage distribués dans douze sites sur le territoire national. Par ailleurs, la fédération de clouds académiques est composée fin 2015 de huit sites (dont six en production) et un planifié pour 2016 avec un total de 2000 cœurs et 100 To de stockage.

\section{IMPLICATIONSSOCIO-ÉCONOMIQUES}

L'utilisation des ressources est accessible aux organismes ou entreprises dans le cadre de projets de recherche communs avec les partenaires du GIS.

\section{DONNÉES}

Stockage : 25 Po

Accessibilité : Via RENATER

Présence dans des réseaux de données : Partenaire français de EGI, Réseau Bases de Données du CNRS

\section{Coût de fonctionnement \\ $2,5 \mathrm{M} €$}

\section{Coût RH 7,33 ETPT}

\section{Dimension internationale}

Le GIS France Grilles est le représentant français dans EGI. Au niveau européen et international, EGl est la plus grande infrastructure distribuée au monde. Elle permet aux communautés scientifiques de partager ressources, données et expertise de façon inégalée. La France en est I'un des membres fondateurs. En 2015, la contribution de la France à EGI s'est élevé à 645 millions d'heures CPU normalisées (KSI2K), soit 13,9\% des ressources de calcul, plaçant la France au ze rang mondial derrière l'Angleterre (15,05\%) et l'Allemagne (14,6\%).

Coordinateur : Yannick Legré, Managing Director

Pays partenaires : $\mathrm{BE}, \mathrm{BG}, \mathrm{CH}, \mathrm{CZ}, \mathrm{EE}, \mathrm{ES}, \mathrm{FI}, \mathrm{FR}, \mathrm{DE}, \mathrm{EL}, \mathrm{HR}, \mathrm{HU}, \mathrm{IL}, \mathrm{IT}, \mathrm{MK}$, NL, PL, PT, RO, SI, SK, SE, TR, UK

Site internet : $\underline{w w w . e g i . e u}$ 


\section{Grid'5000}

Grid'5000 est un instrument scientifique flexible et de grande taille pour le support de la démarche expérimentale dans tous les domaines de l'informatique, en particulier pour les systèmes parallèles et distribués tels que les clouds, le HPC et les systèmes pour le big data.

Grid'5000 est principalement ouvert aux chercheurs en informatique distribuée et parallèle ayant à effectuer des expériences à tous les niveaux de la pile logicielle. De plus les caractéristiques des clusters impliqués et leur connexion rapide à travers RENATER en font une plate-forme de choix pour d'autres communautés scientifiques voulant mettre au point des algorithmes et des applications en vraie grandeur en testant certaines piles logicielles ou certains matériels spécifiques.

Saconception focalisée sur la qualité de l'infrastructure pour le supportd'une bonne démarche expérimentale en informatique plutôt que sur la recherche de puissance de calcul ou de traitement des données en fait un instrument scientifique unique et précieux pour la recherche en informatique.

Par son positionnement (plate-forme expérimentale et non infrastructure de production) et son échelle, Grid'5000 est une plate-forme unique au monde.

\section{IMPLICATIONS SOCIO-ÉCONOMIQUES}

8 startup ont déjà été créées à partir des travaux de recherches effectués sur l'infrastructure : Activeon, Easyvirt, Efficit, Genes Diffusion, Lyatiss/Cloudweaver, Rozzo, Stimergy, Sysfera.

\section{Coût de construction}

$-$

\section{4,8 M€}

Coût de fonctionnement : $0,2 \mathrm{M€}$ (hors renouvellement matériel)

\section{Coût RH}

\section{6,81 ETPT}

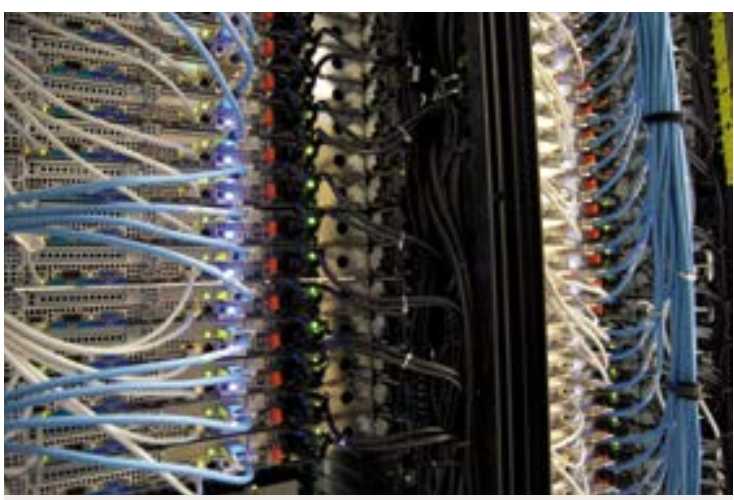

Type de l'infrastructure : IR

Statut de l'infrastructure :

Groupement d'Intérêt Scientifique

\section{Localisation :}

Grenoble

\section{Localisation des autres sites :}

Lille, Luxembourg, Lyon, Nancy, Nantes, Rennes, Sophia-Antipolis

Responsable de l'infrastructure:

Frédéric DESPREZ

\section{Création :}

2012

\section{Tutelles / Partenaires :}

Inria, CNRS, RENATER, CEA, CPU, CDEFI, IMT

\section{Contact en France :}

bureau_gis_g5k@inria.fr

www.grid5000.fr 


\section{Grand Équipement pour la Recherche en Mathématiques}

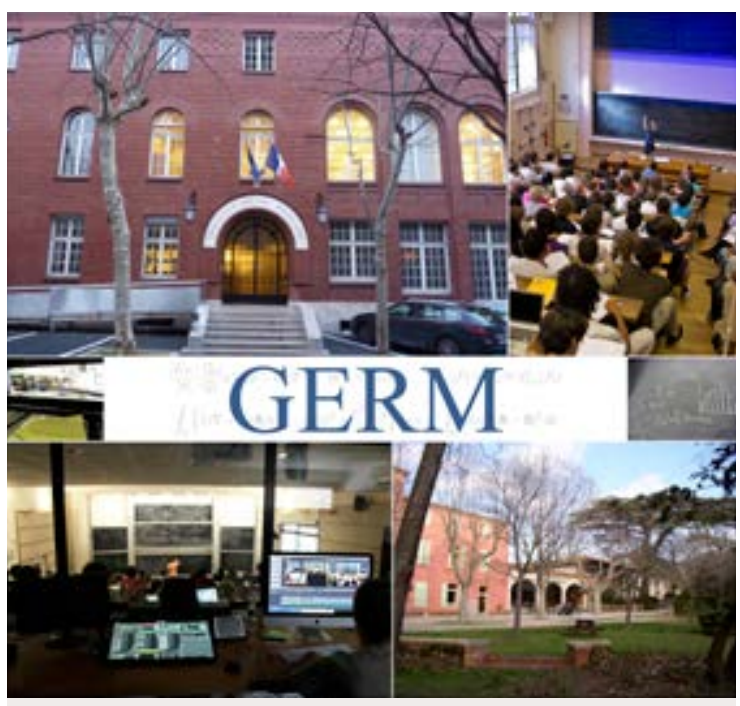

Type de l'infrastructure : Projet

\section{Localisation de l'infrastructure:}

Paris

\section{Localisation des autres sites:}

Île-de-France, Grenoble, Marseille, Nice

\section{Responsable de l'infrastructure :}

Virginie BONNAILLIE-NOËL

Tutelles / Partenaires :

CNRS, UPMC, UGA, UCA

\section{Contact en France :}

virginie.bonnaillie@ens.fr
Les mathématiques françaises sont présentes de la recherche aux frontières du savoir jusqu'aux interactions avec le monde de l'entreprise et la société, et ce au plus haut niveau international. GERM est une infrastructure qui sera pilotée au niveau national et permettra de maintenir cette excellence grâce à différentes missions

- coordonner les centres d'accueil et de rencontres internationaux que sont I'Institut Henri Poincaré (IHP), le Centre International de Rencontres Mathématiques (CIRM) et I'Institut des Hautes Études Scientifiques (IHES) et les maintenir au niveau de la compétition mondiale;

- promouvoir les mathématiques et leurs interactions dans les pays en développement en s'appuyant sur le Centre International de Mathématiques Pures et Appliquées (CIMPA);

- coordonner les interactions de la communauté des mathématiciens avec l'entreprise et la société

- rendre accessible à tous une Bibliothèque Mathématique Audiovisuelle, plateforme évoluée construite sur un corpus de conférences données par des mathématiciens du monde entier lors de leur séjour en France et qui aura toutes les fonctionnalités d'une recherche documentaire de haut niveau sur une base de films catalogués et enrichis de métadonnées;

- renforcer la coordination entre ces différentes institutions pour en faire un outil stable et bien organisé, avec une offre de service complète et coordonnée à destination de la communauté internationale.

GERM deviendra le nœud français pour ces missions.

\section{IMPLICATIONSSOCIO-ÉCONOMIQUES}

GERM offrira aux entreprises, chercheurs et étudiants une visibilité des opportunités dans le domaine des interactions avec les entreprises et organisera des Forums Emploi Maths où se rencontreront étudiants, recruteurs de grands groupes et PME. Il organisera au niveau régional des Semaines d'Études Maths-Entreprises où industriels et chercheurs académiques exploreront des sujets pluritdisciplinaires.

\section{Coût de fonctionnement}

2,2 M€ 


\section{Réseau National de plates-formes de Réalité Virtuelle et Augmentée}

Lors de cette dernière décennie, la réalité virtuelle et la réalité augmentée sont passées d'un stade d'exploration scientifique accompagné d'un développement technologique et d'expérimentation applicatives à celui de la maturité scientifique et de l'évaluation des usages.

L'objectif de RNRVA est d'être le déclencheur de l'étape suivante : le déploiement à large échelle, tout d'abord dans des sciences autres que l'informatique, et ensuite dans différents secteurs industriels. Sa mission est de proposer un ensemble de matériels, de logiciels et surtout de compétences au secteur académique mais aussi au monde de l'entreprise.

La mise en réseau au sein du RNRVA d'un ensemble d'équipements et de compétences extrêmement variés, que ce soit en matériels de restitution multi-sensoriels, en dispositifs d'interaction multimodale et plus généralement en termes d'usages représentera un formidable outil de production scientifique et de valorisation industrielle pour les chercheurs et les ingénieurs. RNRVA sera de nature à renforcer la proximité humaine entre les laboratoires de recherche et le tissu industriel (notamment au sein de l'AFRV) dans le but de produire un impact scientifique puis industriel majeurs.

Les grands défis scientifiques que cette infrastructure permettrait de relever sont très nombreux et par essence pluridisciplinaires. Quelques défis parmi les plus importants sont ceux de la complexité, de la transparence des dispositifs, de la naturalité des interactions et de l'humain virtuel.

\section{Coût de construction \\ $7 \mathrm{M} €$}

\section{Coût RH} 5 ETPT

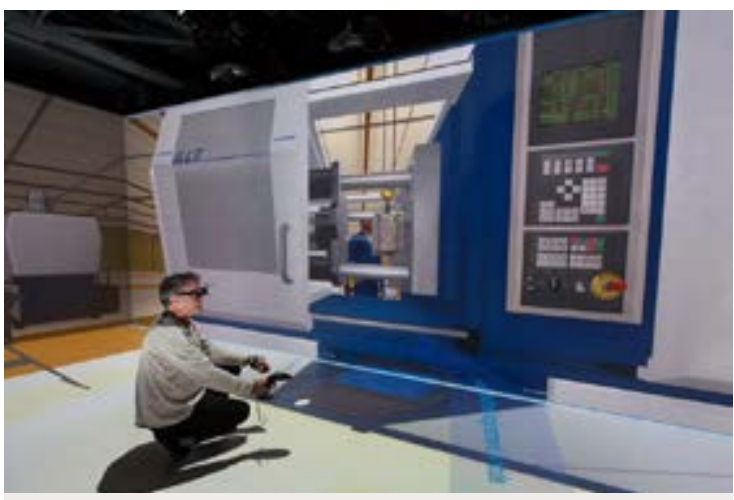

Type de l'infrastructure : Projet

\section{Localisation:}

Bordeaux, Rennes

Localisation des autres sites :

IR distribuée

Responsables de l'infrastructure : Matthieu LÉPINE, Bruno ARNALDI, Pascal GUITTON

Tutelles / Partenaires:

CNRS, Inria, Universités, Grandes Écoles

\section{Contact en France:}

matthieu.lepine@immersion.fr

bruno.arnaldi@irisa.fr

pascal.guitton@labri.fr 

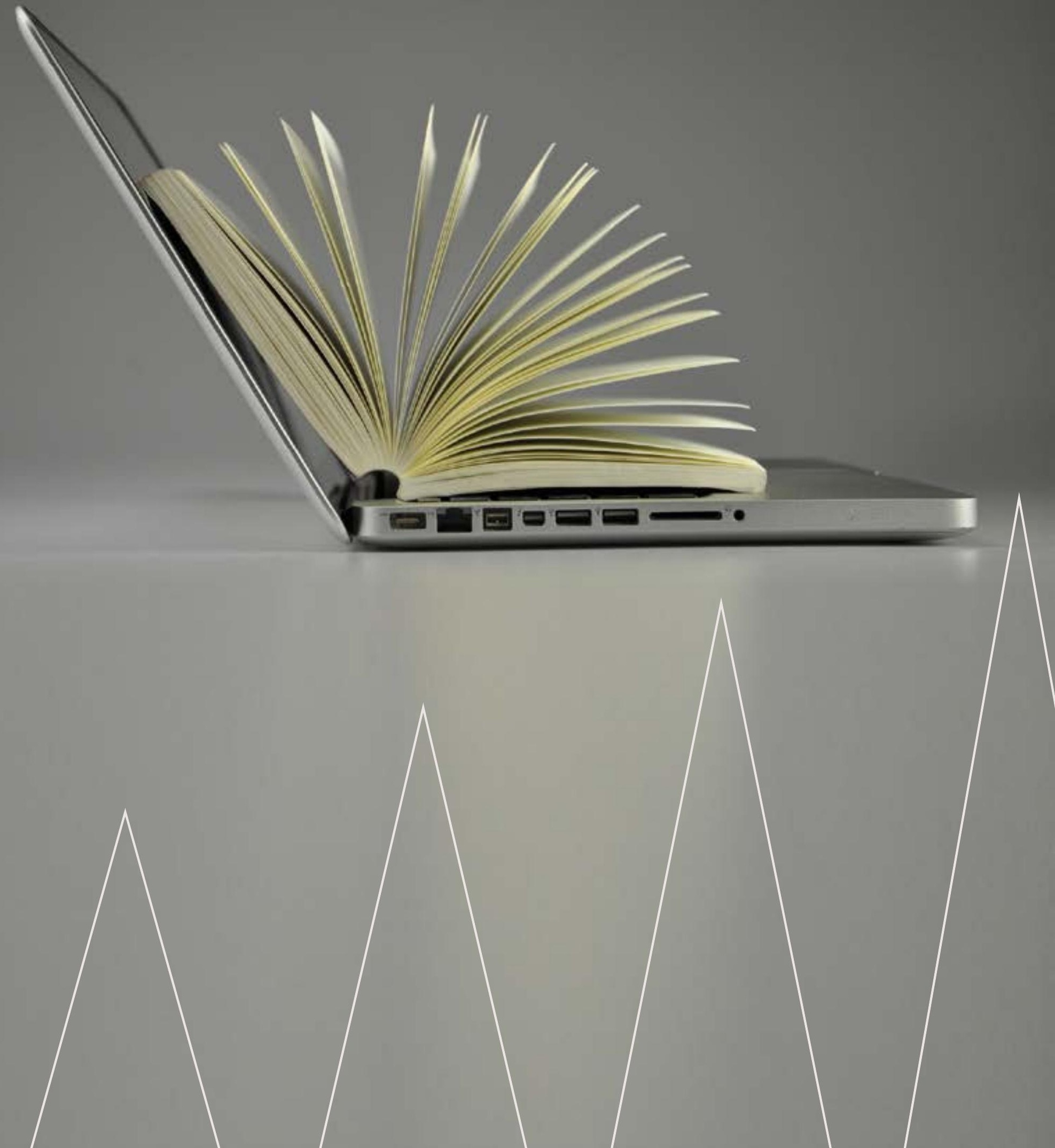
INFORMATION SCIENTIFIQUE ET TECHNIQUE

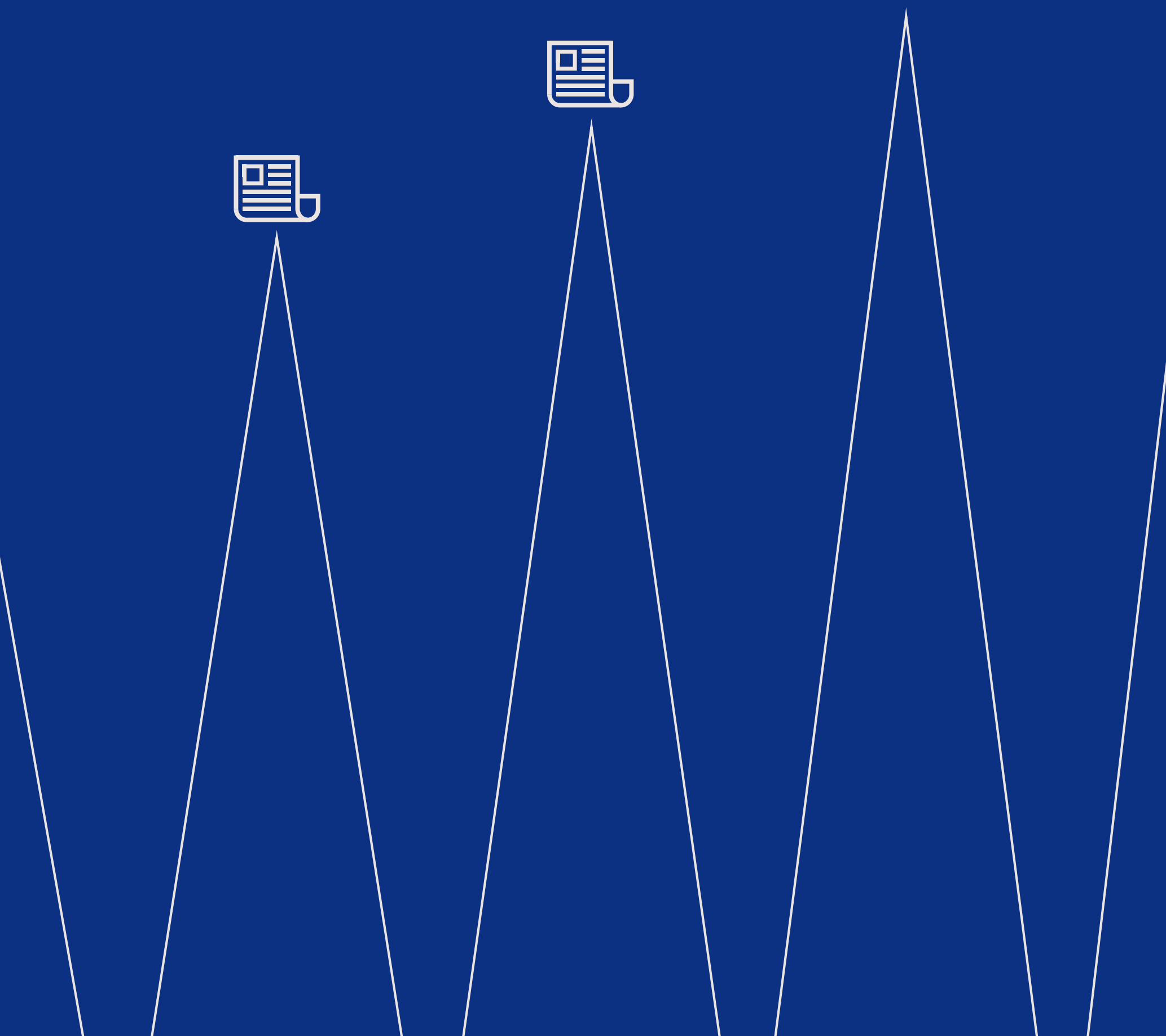




\section{Information scientifique}

\section{et technique}

L'importance des infrastructures en information scientifique et technique (IST) s'est accrue au cours des années 2000, du fait de la numérisation accélérée des publications scientifiques, et du développement des plateformes internationales de diffusion des publications, qui ont facilité la création d'outils scientométriques plus performants.

En France, I'IST est désormais inscrite comme un domaine à part entière doté d'infrastructures indispensables à la pratique scientifique, à la mesure de son importance dans l'économie des systèmes de recherche, avec pour objectifs la structuration d'un secteur très actif mais encore très éclaté, et le développement de l'accès à une offre de ressources scientifiques abondante et de qualité, tout en limitant ses coûts.

\section{1}

\section{DE LA BIBLIOTHĖQUE SCIENTIFIQUE NUMÉRIQUE (BSN) AUX INFRASTRUCTURES HAL, OPEN-EDITION, COLLEX-PERSEE ET NUMEDIF}

Le dispositif national de coordination et de coopération de la Bibliothèque Scientifique Numérique (BSN) a été créé en 2009 pour fournir aux chercheurs une offre en IST d'un niveau d'excellence mondial et améliorer la visibilité de la recherche française. II couvre 9 domaines (acquisitions, signalement, Libre accès, numérisation, archivage pérenne, édition scientifique publique, fourniture de documents, formation, données de la recherche) et en réunit les acteurs (MENESR, organismes, universités, BnF...) et les plateformes (HAL, PERSEE, OpenEdition, NUMEDIF).
La BSN s'est centrée notamment sur l'abonnement aux revues scientifiques, I'acquisition d'archives numériques (programme «PIA » ISTEX de $60 \mathrm{M€}$ ) et les licences nationales (marché Elsevier...).

L'évolution des structures conduit aujourd'hui à identifier, dans la BSN, les plateformes, montées en puissance, qui répondent à l'ensemble des critères requis pour être inscrites comme Infrastructures en Information Scientifique et Technique, sous la supervision de l'Alliance Athéna au nom de l'ensemble des Alliances. La BSN pour sa part forme un des 4 piliers du CODORNUM (Comité d'orientation du numérique de l'ESR).

\section{2}

\section{LES QUATRE PLATEFORMES EN IST : HAL, OPEN-EDITION, NUMEDIF ET COLLEX-PERSEE \\ $\bullet$}

Ces 4 plateformes constituent des ensembles complémentaires "usages-technologies " couvrant le processus de diffusion de la production scientifique. Elles ont en commun la volonté d'ouverture à l'international de la production scientifique française tout en assurant une indépendance technologique. Elles interagissent toutes avec des infrastructures internationales (métadonnées partagées, accords de partenariat, dispositifs de réversion).

2-1 La plateforme HAL : gestion des pré-prints, des multiples versions et archivage de la production scientifique en libre accès

L'archive ouverte HAL (hal.archives-ouvertes.fr) offre le dépôt en libre accès d'articles scientifiques et des données

\section{PROCESSUS DE DIFFUSION DES RÉSULTATS DE LA RECHERCHE}

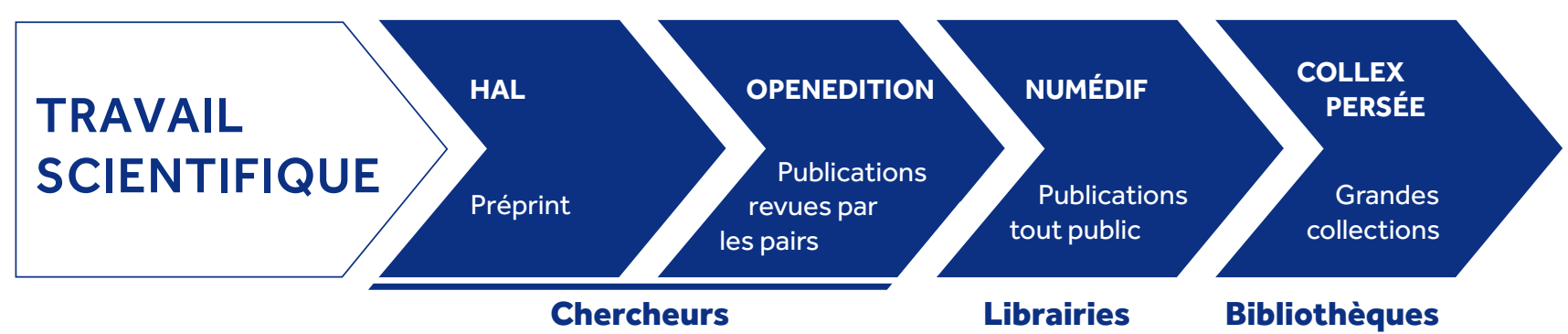


associées, ainsi que de thèses. Créée en 2001 par le CNRS, cette archive a été adoptée par l'ensemble des établissements de I'ESR. En 2006, les établissements de recherche et les universités ont signé un protocole d'accord en vue d'une approche coordonnée, au plan national, pour l'archivage ouvert de la production scientifique. La plateforme HAL est connectée à de nombreux réservoirs internationaux dont ARXIV. Autour de cette plateforme, le Centre pour la Communication Scientifique Directe (CCSD), associant le CNRS, I'INRIA, et l'Université de Lyon, réalise d'autres outils : Sciencesconf.org (colloques), Episciences. org (épi-journaux), Héloïse (droits de diffusion des articles).

\section{2-2 Les plateformes d'OpenEdition : diffusion} en libre accès de la production scientifique éditée et expérimentation de nouvelles formes d'écriture scientifique (revues et ouvrages en SHS avec outils associés).

II s'agit de 4 plateformes portant 420 revues, 1400 carnets de recherche, 2600 livres, liés à plus de 28000 programmes scientifiques. OpenEdition fédère des communautés de recherche internationales ( 50 millions de visites annuelles). Sa visibilité comme infrastructure appuiera, au niveau européen, l'impact des projets de recherche pluridisciplinaire qu'elle diffuse et leur capacité à transférer leurs résultats aux acteurs socio-économiques pour répondre aux défis sociétaux.

Il s'agit ainsi :

- d'améliorer l'accessibilité et la diffusion des publications scientifiques, notamment en SHS;

- de coordonner la transition numérique des acteurs de la publication en SHS;

- d'encourager les bonnes pratiques, d'établir des standards de qualité scientifique et éditoriale;

- de développer des modes de financement durables, transparents, pour la publication en libre accès;

- de soutenir l'émergence de nouvelles méthodes de recherche par la publication numérique.

2-3 NUMEDIF : diffusion et distribution multicanaux La plateforme NUMEDIF résulte d'une collaboration entre le service de diffusion des presses universitaires confié à la FMSH, et une unité de recherche sur l'activité éditoriale de l'Université de Caen. Cette plateforme publique mutualisée de diffusion-distribution multicanaux repose sur la prescription de normes et standards dans le domaine de l'édition et de la diffusion numérique ainsi que sur l'animation d'un réseau (formation, partenariats...).

Elle optimise la circulation des écrits scientifiques et leur mise à disposition tant sous forme numérique (archives, plateformes...) que matérielle (diffusion en librairie et bibliothèques ou auprès des particuliers). Centrée sur le livre en SHS, ses cibles sont les librairies à public universitaire pour la production courante, les bibliothèques universitaires et celles des centres de recherche. Elle est aussi accessible à l'international (vente en ligne).

Une attention spéciale est portée à l'articulation et à I'interopérabilité avec les opérateurs publics en IST : OpenEdition (plateformes), Hal (archives ouvertes), Persée (rétro-numérisation). NUMEDIF est compatible dès à présent avec les standards des plateformes Revues.org, Cairn (revues) et Open Edition Books (livres).

\section{2-4 COLLEX-PERSEE : identification} et consolidation de grandes collections scientifiques accessibles en ligne et dans les grandes bibliothèques

La $4 \mathrm{e}$ infrastructure coordonne, dans un dispositif qui optimise leur action, des acteurs indispensables mais moins visibles dans le processus de production comme de diffusion de la recherche : les bibliothèques. CollEx-PERSEE réunit les bibliothèques de référence dans leurs disciplines (BDIC, BNUS, BIU de la Sorbonne, GED Condorcet, BULAC, BIU Santé, bibliothèques de I'INHA, de l'Observatoire de Paris, du MNHN...), la BnF, ainsi que des opérateurs en IST ( $A B E S, C T L e s, ~ I N I S T$ ), et Huma-Num, en vue de développer des services nouveaux, articulés aux collections, en lien direct avec leurs utilisateurs-chercheurs.

Appuyée sur la plateforme de numérisation PERSEE, cette infrastructure permet de gérer et de valoriser les grands fonds scientifiques et patrimoniaux. Elle est aussi un outil majeur des politiques d'acquisition et de conservation partagée, qui répondent à des logiques à la fois disciplinaires et de stratégies territoriales. 
LISTE DES INFRASTRUCTURES DE RECHERCHE

DANS LE DOMAINE INFORMATION SCIENTIFIQUE ET TECHNIQUE

\begin{tabular}{|c|c|c|c|}
\hline CATÉGORIE & NOM & NOM COMPLET & ESFRI \\
\hline IR & COLLEX PERSEE & $\begin{array}{l}\text { Collections d'excellence pour la Recherche - } \\
\text { Persée }\end{array}$ & \\
\hline IR & HAL, CCSD & $\begin{array}{l}\text { Archive ouverte HAL (Hyper Article en Ligne), } \\
\text { Centre pour la Communication Scientifique Directe }\end{array}$ & \\
\hline IR & NUMEDIF & $\begin{array}{l}\text { NUMérique pour l'ÉDItion et la DIFFusion } \\
\text { de la production scientifique }\end{array}$ & \\
\hline IR & OpenEdition ${ }^{1}$ & $\begin{array}{l}\text { Plateformes de publication électronique } \\
\text { en sciences humaines et sociales }\end{array}$ & \\
\hline
\end{tabular}

1 Relève également du secteur « Sciences Humaines et Sociales». 


\section{COLLEX-PERSEE}

\section{Collections d'excellence pour la Recherche - Persée}

CollEx-PERSEE est une infrastructure distribuée créée pour rapprocher les chercheurs des gisements documentaires scientifiques et leur en faciliter l'usage. Elle permet un accès national mieux coordonné et mutualisé aux ressources documentaires, négociées par communautés (inter)disciplinaires, ainsi qu'aux corpus patrimoniaux et scientifiques, imprimés ou dématérialisés. Elle porte la politique de l'ESR en matière de numérisation et de conservation partagée et participe au développement des Humanités numériques en lien avec Huma-Num.

Elle articule les communautés scientifiques (représentées notamment par l'Alliance Athéna) et les grands acteurs documentaires réunis en GIS (bibliothèques universitaires de référence, organismes de recherche, BnF, réseaux documentaires; Persée; opérateurs de l'Etat: ABES, CTLes). Sa mission est d'optimiser l'environnement de recherche, en consolidant les collections documentaires et en constituant des corpus de matériaux de recherche uniques et attractifs à l'international, en optimisant leur référencement ainsi que leur utilisation par les chercheurs et équipes de recherche. Elle conçoit et promeut une offre de services ciblés au plus près des besoins de la recherche (collecte de matériaux pour les projets de recherche, signalement, référencement web et cartographie des collections d'excellence, accessibilité et fourniture à distance, services conçus avec les chercheurs).

PERSEE en constitue l'opérateur central tant pour organiser des programmes de numérisation avec les chercheurs, que comme plateforme de diffusion et d'accès.

\section{IMPLICATIONSSOCIO-ÉCONOMIQUES}

CollEx offre des services d'accès à l'information scientifique et technique également en direction des travailleurs indépendants, des TPE et PME qui n'ont pas de moyens documentaires importants (notamment en droit, médecine, économie, gestion, sciences de l'ingénieur).

\section{Coût de construction}

$0,115 \mathrm{M€}$

Coût de fonctionnement : $8,5 \mathrm{M€}$

\section{Coût RH}

-

Coordination : 0,5 ETPT.

Les RH globales de CollEx

correspondent aux personnels des établissements et des

services d'IST constituant I'infrastructure répartie.

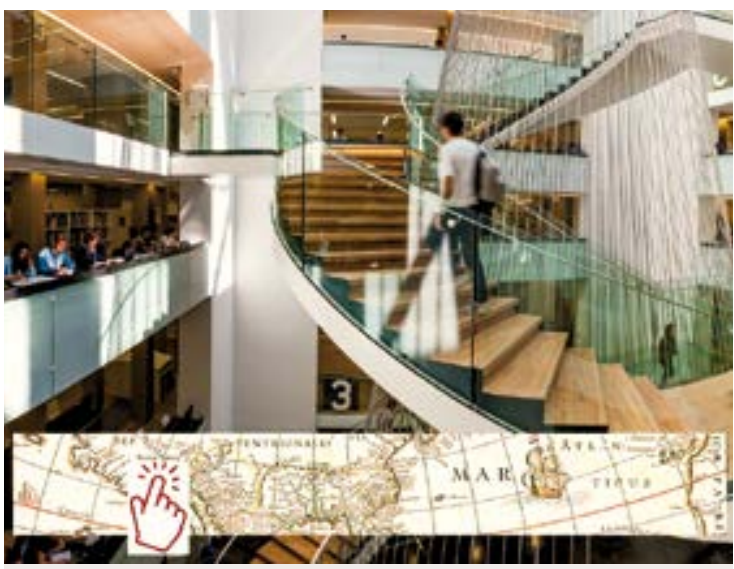

Type de I'infrastructure : IR

\section{Localisation :}

Paris, Lyon

Localisation des autres sites :

Aix, Bordeaux, Bussy-Saint-Georges, Caen, Grenoble, Lille, Lyon, Montpellier, Nancy, Poitiers, Strasbourg, Toulouse

Responsables de l'infrastructure : Michel MARIAN, Françoise THIBAULT, Nathalie FARGIER

\section{Création: Exploitation: \\ 2014 2014}

\section{Tutelles / Partenaires:}

MENESR, Alliance Athéna, Univ. Lyon, CNRS, ENS Lyon

\section{Contact en France :}

collex@recherche.gouv.fr

\section{www.collex.eu}




\section{Archive ouverte HAL (Hyper Article en Ligne), Centre pour la Communication Scientifique Directe}

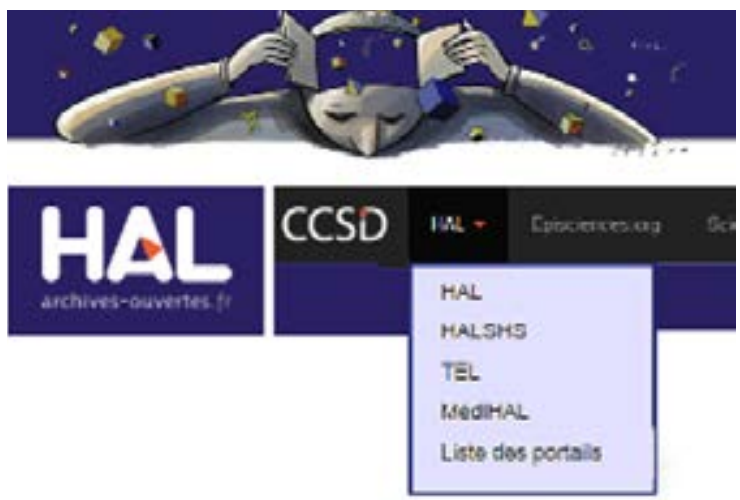

Type de l'infrastructure : IR

Statut de l'infrastructure:

Unité mixte de service

Localisation de l'infrastructure:

Villeurbanne

Responsable de l'infrastructure :

Christine BERTHAUD

Création :

Exploitation :

2000

2001

Tutelles / Partenaires :

CNRS, Inria, Univ. Lyon

\section{Contact en France :}

direction@ccsd.cnrs.fr

\section{hal.archives-ouvertes.fr}

HAL est une plateforme multidisciplinaire d'archives ouvertes utilisée par l'ensemble des membres de la communauté scientifique, chercheurs, enseignants-chercheurs, rattachés à un établissement public à caractère scientifique et technologique (EPST), à une université, ou à une grande école. HAL est interconnectée aux grands serveurs internationaux ArXiv, Pubmed Central, RepEc, OpenAire.

HAL participe à la diffusion en accès ouvert de documents produits par la recherche (articles publiés ou non dans des revues à comité de lecture, communications scientifiques...) concernant 380 champs disciplinaires répartis en 13 grands domaines scientifiques. Sa stratégie s'inscrit dans le mouvement international de l'Open Access et de H2020. Les dépôts font l'objet d'un contrôle qualité des données - conformité technique des formats de fichiers, cohérence texte/notice (titre, auteurs...), contrôle des droits de diffusion - et d'une vérification de la nature scientifique du document. C'est la plateforme commune et partagée par l'ensemble de l'ESR dans le cadre d'une convention inter-établissement (2013).

HAL offre aux établissements qui veulent créer une archive ouverte (AO) institutionnelle tous les outils et fonctionnalités (portail) pour mettre en œuvre ce projet. HAL propose des solutions techniques aux AO qui reversent leur contenu. HAL réalise l'archivage pérenne avec le CINES. Hal propose une plateforme d'accès ouvert à tous ses référentiels. HAL développe des interconnexions avec les grands projets internationaux similaires.

\section{DONNÉES}

Présence dans des réseaux de données : OpenAire, DARIAH-EU, PubMedCentral

\section{Coût de construction \\ 0,15 M€ \\ Coût de fonctionnement : 0,09 M€}

\section{Coût RH}

14 ETP, soit 872 k€

\section{Dimension internationale}

ArXiv, site miroir français géré par le CCSD

Coordinateur : Cornell university, USA

Pays partenaires : archive internationale

Site internet : arxiv.org 


\section{NUMEDIF (NUMérique pour l'ÉDItion et la DIFFusion de la production scientifique)}

L'infrastructure NUMEDIF estau service del'activité éditoriale de l'ensemble des établissements publics d'enseignement supérieur et de recherche. Elle s'attache à la mise au point et à la dissémination d'un écosystème normé de production numérique de contenus scientifiques structurés interopérables, et à la construction d'un appareil spécifique de diffusion-distribution.

Elle vise à garantir la circulation des écrits scientifiques et leur mise à disposition optimale tant sous forme dématérialisée (archives, plateformes...) que matérielle (livres ou revues diffusés en librairie, auprès des bibliothèques ou des particuliers) et doit améliorer la visibilité de l'édition scientifique publique française à l'international.

Elle permet l'alimentation pour un coût résiduel des plateformes «nationales»: Open Edition, Cairn... Elle est garante de la pérennité des données. L'utilisation d'Unicode et la normalisation documentée des structures (XML-TEI) permet l'archivage par le Centre informatique National de l'Enseignement supérieur (CINES).

Ses missions et services portent sur:

- la constitution des fonds de contenus édités, normalisés, interopérables, bâtis sur le modèle du single source publishing;

- I'articulation des échelles de diffusion nécessaires (librairies et publics spécialisés, mises en place plus massives en librairies à destination d'un public large, diffusion en ligne, diffusion de formes numériques détachables, diffusion payante, open access dont OpenEdition);

- un service de veille et de formation;

- I'industrie du logiciel, le développement d'environnement de saisie et de traitement de flux structurés normés, le développement d'automates de composition de flux éditorialisés pour la production d'epubs et l'alimentation des plateformes de diffusion;

- les diffuseurs-distributeurs, développement de flux mixtes TEI/ONIX pour le référencement et l'optimisation des diffusions multisupports, pour l'alimentation de circuits de print-on-demand.

\section{Coût de construction}

0,92 M€

Coût de fonctionnement : $0,75 \mathrm{M€}$

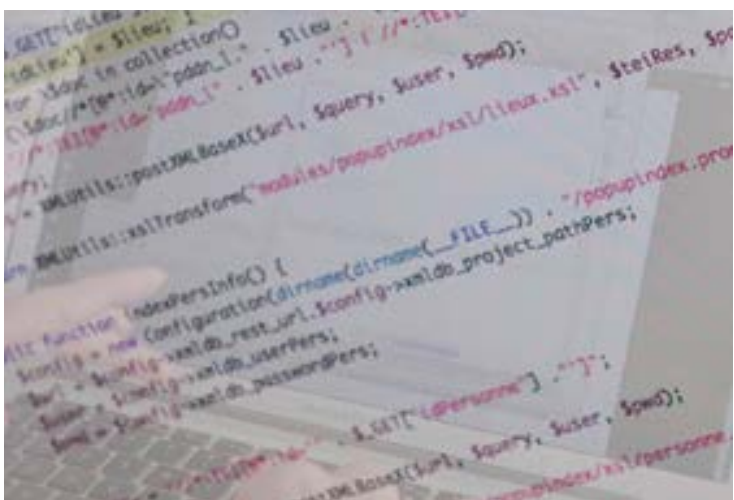

Type de l'infrastructure : IR

\section{Localisation:}

Paris

Localisation des autres sites:

Caen

Responsable de l'infrastructure:

Pascal BULEON

Création: Exploitation:

2015

2015

Tutelles / Partenaires :

FMSH,MRSH, CNRS, UNICAEN

Contact en France:

numedif@msh-paris.fr

\section{numedif.fr}




\section{OPENEDITION @openEdition}

\section{OpenEdition. Plateformes de publication électronique en sciences humaines et sociales}

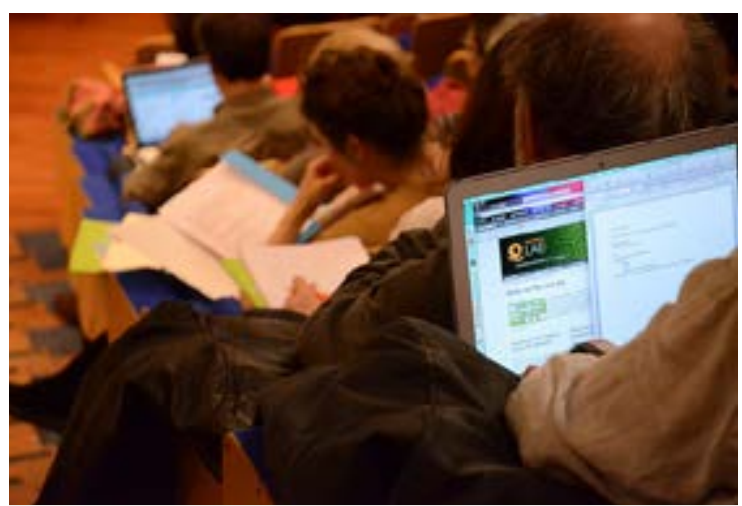

Type de l'infrastructure: Unité mixte de services

\section{Statut de l'infrastructure :}

Unité mixte de services

Localisation de l'infrastructure:

Marseille

\section{Localisation des autres sites :}

Paris

Responsable de l'infrastructure : Marin DACOS

\section{Création :}

Exploitation :

2007

2007

\section{Tutelles / Partenaires :}

AMU, BSN, CNRS, DARIAH, EHESS,

Univ. Avignon

\section{Contact en France :}

contact@openedition.org

\section{www.openedition.org}

OpenEdition est une infrastructure complète d'édition électronique au service de la communication scientifique en sciences humaines et sociales, mise en œuvre par l'UMS Cléo (Centre pour l'édition électronique ouverte). Elle rassemble quatre plateformes complémentaires dédiées respectivement aux collections de livres avec OpenEdition Books, aux revues avec Revues.org, aux blogs de recherche avec Hypothèses et aux annonces scientifiques avec Calenda. L'ensemble de ces plateformes reçoit 3 millions de visites mensuelles provenant du monde entier.

La mission d'OpenEdition est de favoriser le développement d'une édition électronique de haut niveau et d'envergure internationale. Sa création a pour origine une idée simple: dispersés, les efforts des divers acteurs de l'édition scientifique sont noyés dans le vaste océan du Web. Regroupés autour d'un portail, d'importantes économies d'échelles sont possibles. Une masse critique de contenus, de technologies et de compétences permet de dépasser la simple mise en ligne de documents scientifiques, et ce en misant sur la formation à de nouveaux usages éditoriaux, sur l'internationalisation, sur un modèle économique durable et sur des innovations technologiques régulières.

\section{IMPLICATIONSSOCIO-ÉCONOMIQUES}

Dans le cadre d'un PIA, le Cléo est partenaire de deux sociétés: Demain un autre jour (DUAJ) et Qwam Content intelligence (QUAM CI) sur des questions de text mining et d'enrichissement automatique.

www.demainunautrejour.com www.qwamci.com

\section{DONNÉES}

Estimation du flux de données : Trafic de données : 130 To/an Stockage : 6 To

Présence dans des réseaux de données : référencement dans les bases OpenAIRE, OAPEN, DOAB (Directory of Open Access Books), DOAJ (Directory of Open Access Journals), Base (Bielefield Academic Search Engine), EZB (Elektronische Zeitschriftenbibliothek), Google Scholar, Journal TOCs, Mir@bel, New Jour, Sudoc, Isidore (Huma-Num), WorldCat (OCLC)

Et également, en bibliothèque : AtoZ (EBSCO), 360 Core (Serials Solutions), SFX (Exlibris), LinkSolver (Ovid), EBSCO Discovery Service, Summon (Proquest), Primo Central (Exlibris)

\section{Coût de construction \\ 4 M€ \\ Coût de fonctionnement : $3 \mathrm{M€}$}

\section{Coût RH}

45 ETPT

\section{Dimension internationale \\ En construction sous le nom OPERAS. \\ Coordinateur : Pierre Mounier \\ Pays partenaires : DE, IT, CA, PT, NL, EL, UK \\ Site internet: operas.hypotheses.org}


INFORMATION SCIENTIFIQUE ET TECHNIQUE 

ANNEXES 


\section{Annexe 1}

\section{LE PROCESSUS DE MISE À JOUR DE LA STRATÉGIE FRANÇAISE}

Les précédentes éditions de la stratégie nationale des infrastructures de recherche ont été publiées en 2008 et 2012. La présente mise à jour 2016 est le fruit d'un processus collectif largement renouvelé, avec plusieurs temps forts, dont les Alliances et les organismes de recherche ont été les acteurs principaux avec le soutien constant de la DGRI. Une journée de lancement a eu lieu le 7 juillet 2014 pour en définir le cadre : l'articulation avec la mise à jour européenne (roadmap ESFRI), la mise en place de groupes de coordination (GC) thématiques Alliances/Organismes/ Ministère et d'un groupe projet DGRI/animateurs des GC. Dans chaque domaine, les infrastructures candidates ont été identifiées par le GC, puis sollicitées au printemps 2015 sous la forme d'un questionnaire détaillé. Des fiches en ont été extraites puis validées par les tutelles. Enfin, des synthèses thématiques, correspondant à celles de ce document, ont été préparées par les GC.

Ces fiches et ces synthèses ont ensuite été présentées au Haut Conseil des Très Grandes Infrastructures de Recherche (HC-TGIR) en juillet 2015. Cette instance indépendante, rassemblant 13 experts de tous domaines, ayant une longue expérience des grandes infrastructures et des grands projets de recherche, a analysé le paysage ainsi présenté et l'ensemble des fiches. Son rapport a été finalisé en octobre 2015.

Sur la base de cette analyse, le Comité Directeur des Très Grandes Infrastructures de Recherche (CD-TGIR) a arrêté la liste exhaustive des infrastructures officiellement retenues par la France. Cette instance, présidée par le directeur de la DGRI et rassemblant les présidents des Alliances, du CNRS et du CEA a ainsi fixé le paysage français, y compris des projets officiellement reconnus.

Enfin, la présentation de ce paysage est l'objet du présent document, ainsi que d'un site web entièrement nouveau, et dans lequel les évolutions pourront être suivies au cours du temps.

La publication de ces documents a été marquée par une journée nationale de présentation de la nouvelle stratégie le 24 mars 2016 au MENESR, associée à une journée consacrée au modèle économique des infrastructures le 25 mars 2016. 


\section{Annexe 2}

INFRASTRUCTURES DE RECHERCHE 2016 :

LISTE DES OI, TGIR, IR, PROJETS

\begin{tabular}{|c|c|c|c|}
\hline CATÉGORIE & NOM & NOM COMPLET & ESFRI \\
\hline \multicolumn{4}{|c|}{ SCIENCES HUMAINES ET SOCIALES } \\
\hline TGIR & Huma-Num & Humanités Numériques & DARIAH (2006) \\
\hline TGIR & Progedo & PROduction et GEstion de DOnnées & $\begin{array}{l}\text { ESS (2006) } \\
\text { CESSDA (2006) } \\
\text { SHARE (2006) } \\
\text { GGP (2016) }\end{array}$ \\
\hline IR & OpenEdition $^{1}$ & $\begin{array}{l}\text { Edition électronique ouverte en Sciences } \\
\text { humaines et sociales }\end{array}$ & \\
\hline IR & $\mathrm{RnMSH}$ & $\begin{array}{l}\text { Réseau national des Maisons des Sciences } \\
\text { de l'Homme }\end{array}$ & \\
\hline Projet & $E R I H S-F R^{2}$ & $\begin{array}{l}\text { European Research Infrastructure for Heritage } \\
\text { Science }\end{array}$ & ERIHS (2016) \\
\hline \multicolumn{4}{|c|}{ SCIENCES DU SYSTÈME TERRE ET ENVIRONNEMENT } \\
\hline Ol & CEPMMT & $\begin{array}{l}\text { Centre Européen pour les Prévisions } \\
\text { Météorologiques à Moyen Terme }\end{array}$ & \\
\hline TGIR & Concordia & Base antarctique franco-italienne & \\
\hline TGIR & ECORD/IODP & $\begin{array}{l}\text { Programme international de forage profond } \\
\text { en mer/European Consortium for Ocean Drilling } \\
\text { Research/International Ocean Discovery Program }\end{array}$ & \\
\hline TGIR & EURO-ARGO & $\begin{array}{l}\text { Réseau in-situ global d'observation des océans/ } \\
\text { European contribution to Argo programme }\end{array}$ & $\begin{array}{l}\text { EURO-ARGO } \\
(2006)\end{array}$ \\
\hline TGIR & FOF & Flotte Océanographique Française & \\
\hline TGIR & ICOS & $\begin{array}{l}\text { Système Intégré d'Observation du Carbone/ } \\
\text { Integrated Carbon Observation System }\end{array}$ & $\operatorname{ICOS}(2006)$ \\
\hline IR & ACTRIS - FR & $\begin{array}{l}\text { Aerosol, Cloud and Trace Gases Research } \\
\text { Infrastructure - France }\end{array}$ & ACTRIS (2016) \\
\hline IR & ANAEE - FR & $\begin{array}{l}\text { Analyses et Expérimentations sur les Ecosystèmes } \\
\text { - France }\end{array}$ & ANAEE (2010) \\
\hline IR & ClimERI-FR & $\begin{array}{l}\text { Infrastructure nationale de modélisation } \\
\text { du système climatique de la Terre/Earth's } \\
\text { Climate system Modelling }\end{array}$ & \\
\hline
\end{tabular}

1 Relève également du secteur "Information Scientifique et Technique ».

2 Relève également du secteur "Sciences de la Matière et Ingénierie ». 


\begin{tabular}{|c|c|c|c|}
\hline CATÉGORIE & NOM & NOM COMPLET & ESFRI \\
\hline IR & ECOSCOPE & $\begin{array}{l}\text { Pôle de données d'observation pour la recherche } \\
\text { sur la biodiversité }\end{array}$ & \\
\hline IR & ECOTRONS & Ecotrons & ANAEE (2010) \\
\hline IR & $E M B R C-F R^{3}$ & $\begin{array}{l}\text { Centre National de Ressources Biologiques } \\
\text { Marines }\end{array}$ & $E M B R C$ (2008) \\
\hline IR & EMPHASIS France ${ }^{4}$ & $\begin{array}{l}\text { European Multi-environment Plant pHenomics } \\
\text { And Simulation InfraStructure -France }\end{array}$ & EMPHASIS (2016) \\
\hline IR & EMSO - France & $\begin{array}{l}\text { European Multidisciplinary Seafloor and } \\
\text { water column Observatory - France }\end{array}$ & EMSO (2006) \\
\hline IR & IAGOS - France & $\begin{array}{l}\text { Instruments de mesure embarqués sur avions } \\
\text { pour l'observation globale/ln-service Aircraft } \\
\text { for Global Observing System }\end{array}$ & IAGOS (2006) \\
\hline IR & I-LI-CO & Infrastructure LIttorale et COtière & \\
\hline IR & OZCAR & $\begin{array}{l}\text { Observatoire de la Zone Critique, Applications, } \\
\text { Recherche }\end{array}$ & \\
\hline IR & RARE & Ressources Agronomiques pour la Recherche & \\
\hline IR & RECOLNAT & Réseau des Collections Naturalistes françaises & \\
\hline IR & RESIF/EPOS & $\begin{array}{l}\text { Réseau sismologique et géodésique français/ } \\
\text { European Plate Observing System }\end{array}$ & EPOS (2008) \\
\hline IR & SAFIRE & $\begin{array}{l}\text { Service des Avions Français Instrumentés } \\
\text { pour la Recherche en Environnement }\end{array}$ & \\
\hline Projet & $I B I S B A-F R^{5}$ & $\begin{array}{l}\text { Industrial Biotechnology Innovation } \\
\text { and Synthetic Biology Accelerator }\end{array}$ & \\
\hline Projet & Pole de données & Pôle de données et services pour le Système Terre & \\
\hline \multicolumn{4}{|c|}{ ENER G I E } \\
\hline IR & FR-SOLARIS & $\begin{array}{l}\text { Infrastructure de Recherche française sur le solaire } \\
\text { thermique concentré/Solar Thermal Research } \\
\text { Infrastructure for Concentrated Solar Power }\end{array}$ & $\begin{array}{l}\text { EU-SOLARIS } \\
(2010)\end{array}$ \\
\hline IR & WEST & W(Tungsten) Environment for Steady-state Tokamaks & \\
\hline Projet & ECCSEL-FR & $\begin{array}{l}\text { European Carbon Dioxide Capture and StoragE } \\
\text { Laboratory Infrastructure }\end{array}$ & ECCSEL (2008) \\
\hline Projet & SOPHIRA & $\begin{array}{l}\text { Infrastructure pour la recherche sur le solaire } \\
\text { photovoltaïque/SOlar PHotovoltaic Research } \\
\text { Infrastructure }\end{array}$ & \\
\hline Projet & Theorem & $\begin{array}{l}\text { Réseau de Moyens d'Essais en Hydrodynamique } \\
\text { pour les Énergies Marines Renouvelables }\end{array}$ & Marinerg-i (2016) \\
\hline
\end{tabular}

\footnotetext{
3 Relève également du secteur "Biologie et Santé" ".

4 Relève également du secteur "Biologie et Santé ".

5 Relève également du secteur "Biologie et Santé ».
} 


\begin{tabular}{|c|c|c|c|}
\hline CATÉCORIE & NOM & NOM COMPLET & ESFRI \\
\hline \multicolumn{4}{|c|}{ BIOLOGIE ET SANTÉ } \\
\hline OI & EMBL & $\begin{array}{l}\text { Laboratoire Européen de Biologie Moléculaire/ } \\
\text { European Molecular Biology Laboratory }\end{array}$ & \\
\hline OI & EMBC & $\begin{array}{l}\text { Conférence Européenne de Biologie Moléculaire/ } \\
\text { European Molecular Biology Conference }\end{array}$ & \\
\hline IR & BIOBANQUES & Infrastructure nationale BIOBANQUES & $\begin{array}{l}\text { BBMRI (2006) + } \\
\text { MIRRI (2010) }\end{array}$ \\
\hline IR & $\begin{array}{l}\text { CELPHEDIA/ } \\
\text { PHENOMIN }\end{array}$ & $\begin{array}{l}\text { Infrastructure Nationale en phénogénomique } \\
\text { de la souris }\end{array}$ & $\begin{array}{l}\text { INFRAFONTIER } \\
(2006)\end{array}$ \\
\hline IR & CELPHEDIA/ TEFOR & $\begin{array}{l}\text { Trangénèse pour les Etudes Fonctionnelles } \\
\text { chez les ORganismes modèles }\end{array}$ & \\
\hline IR & CONSTANCES & $\begin{array}{l}\text { Cohorte des consultants des Centres d'examens } \\
\text { de santé }\end{array}$ & \\
\hline IR & CRB Anim & $\begin{array}{l}\text { Centres de Ressources Biologiques pour } \\
\text { les animaux domestiques }\end{array}$ & \\
\hline IR & E-CellFrance & $\begin{array}{l}\text { Plateforme nationale pour la médecine } \\
\text { régénératrice basée sur les cellules souches } \\
\text { mesenchymateuses adultes }\end{array}$ & \\
\hline IR & EMBRC-France ${ }^{6}$ & $\begin{array}{l}\text { Centre National de Ressources Biologiques } \\
\text { Marines }\end{array}$ & $E M B R C$ (2008) \\
\hline IR & EMPHASIS France $^{7}$ & $\begin{array}{l}\text { European Multi-environment Plant pHenomics } \\
\text { And Simulation InfraStructure }\end{array}$ & \\
\hline IR & $\mathrm{FBI}$ & France-Biolmaging & EUBIO (2008) \\
\hline IR & F-CRIN & $\begin{array}{l}\text { Plateforme Nationale d'Infrastructures de } \\
\text { recherche Clinique/French Clinical Research } \\
\text { Infrastructure Network }\end{array}$ & ECRIN (2006) \\
\hline IR & FLI & France Life Imaging & EUBIO (2008) \\
\hline IR & France Génomique & $\begin{array}{l}\text { Infrastructure nationale de génomique } \\
\text { et bioinformatique associée }\end{array}$ & \\
\hline IR & Fr-Hadron & France HADRON & \\
\hline IR & FRISBI & $\begin{array}{l}\text { Infrastructure Française pour la Biologie } \\
\text { Structurale Integrée/French Infrastructure } \\
\text { for Integrated Structural Biology }\end{array}$ & INSTRUCT (2006) \\
\hline IR & HIDDEN & $\begin{array}{l}\text { Infrastructure de recherche dédiée aux maladies } \\
\text { hautement infectieuses/Highly Infectious } \\
\text { Diseases Dedicated Infrastructure ExtensioN }\end{array}$ & ERHINA (2008) \\
\hline IR & IDMIT & $\begin{array}{l}\text { Infrastructure nationale pour la modélisation } \\
\text { des maladies infectieuses humaines et } \\
\text { les thérapies innovantes }\end{array}$ & \\
\hline IR & IFB & Institut Français de Bioinformatique & ELIXIR (2006) \\
\hline
\end{tabular}

6 Relève également du secteur "Sciences du système Terre et Environnement ".

7 Relève également du secteur "Sciences du système Terre et Environnement ». 


\begin{tabular}{|c|c|c|c|}
\hline CATÉGORIE & NOM & NOM COMPLET & ESFRI \\
\hline IR & INGESTEM & $\begin{array}{l}\text { Infrastructure Nationale d'ingénierie des cellules } \\
\text { souches et des tissus }\end{array}$ & \\
\hline IR & MétaboHub & $\begin{array}{l}\text { Infrastructure française distribuée pour } \\
\text { la métabolomique dédiée à l'innovation, } \\
\text { à la formation et au transfert de technologie }\end{array}$ & \\
\hline IR & NEURATRIS & $\begin{array}{l}\text { Infrastructure de Recherche Translationnelle } \\
\text { pour les Biothérapies en Neurosciences }\end{array}$ & EATRIS (2006) \\
\hline IR & PGT & $\begin{array}{l}\text { Consortium Préindustriel des vecteurs } \\
\text { de Thérapie Génique }\end{array}$ & \\
\hline IR & PROFI & $\begin{array}{l}\text { Infrastructure Française de Protéomique/ } \\
\text { Proteomics French Infrastructure }\end{array}$ & \\
\hline Projet & $I B I S B A-F R^{8}$ & $\begin{array}{l}\text { Industrial Biotechnology Innovation } \\
\text { and Synthetic Biology Accelerator }\end{array}$ & \\
\hline \multicolumn{4}{|c|}{ SCIENCES DE LA MATIÈRE ET INGÉNIERIE } \\
\hline TGIR & ESRF & $\begin{array}{l}\text { Source Européenne de Rayonnement } \\
\text { Synchrotron/European Synchrotron } \\
\text { Radiation Facility }\end{array}$ & $\begin{array}{l}\text { ESRF Upgrade Ph } 1 \\
\text { (2006) } \\
\text { ESRF Upgrade Ph } 2 \\
\text { (2016) }\end{array}$ \\
\hline TGIR & ESS & European Spallation Source & ESS (2006) \\
\hline TGIR & ILL & Institut Max von Laue - Paul Langevin & $\begin{array}{l}\text { ILL Upgrade Ph } 1 \\
\text { (2006) }\end{array}$ \\
\hline TGIR & Orphée/LLB & ORPHEE/Laboratoire Léon Brillouin & \\
\hline TGIR & Soleil & $\begin{array}{l}\text { Source Optimisée de Lumière d'Energie } \\
\text { Intermédiaire du LURE }\end{array}$ & \\
\hline TGIR & XFEL & European X-ray Free Electron Laser & XFEL (2006) \\
\hline IR & EMIR & $\begin{array}{l}\text { Fédération des Accélérateurs pour les Études } \\
\text { des Matériaux sous Irradiation }\end{array}$ & \\
\hline IR & FT-ICR & $\begin{array}{l}\text { Réseau national de spectrométrie de masse FT-ICR } \\
\text { à très haut champ }\end{array}$ & \\
\hline IR & LNCMI & $\begin{array}{l}\text { Laboratoire National des Champs Magnétiques } \\
\text { Intenses }\end{array}$ & EMFL (2008) \\
\hline IR & LULI-APOLLON & Laboratoire d'Utilisation des Lasers Intenses & \\
\hline IR & METSA & $\begin{array}{l}\text { Microscopie Electronique en Transmission } \\
\text { et Sonde Atomique }\end{array}$ & \\
\hline IR & PETAL & PETAwatt Aquitaine Laser & \\
\hline IR & $\mathrm{RMN}$ & $\begin{array}{l}\text { Résonance Magnétique Nucléaire à Très Hauts } \\
\text { Champs }\end{array}$ & \\
\hline IR & Renard & REseau NAtional de Rpe interDisciplinaire & \\
\hline
\end{tabular}

8 Relève également du secteur "Sciences du système Terre et Environnement ». 


\begin{tabular}{|c|c|c|c|}
\hline CATÉGORIE & NOM & NOM COMPLET & ESFRI \\
\hline IR & RENATECH & $\begin{array}{l}\text { Réseau NAtional des grandes centrales } \\
\text { de TECHnologies }\end{array}$ & \\
\hline Projet & $E R I H S-F R^{9}$ & $\begin{array}{l}\text { European Research Infrastructure for Heritage } \\
\text { Science }\end{array}$ & ERIHS (2016) \\
\hline \multicolumn{4}{|c|}{ ASTRONOMIE ET ASTROPHYSIQUE } \\
\hline \multirow[t]{4}{*}{ Ol } & ESO & European Southern Observatory & \\
\hline & ESO ALMA & Atacama Large Millimeter/Submillimiter Array & \\
\hline & ESO EELT & European Extremely Large Telescope & E-ELT (2006) \\
\hline & ESO LSP & La Silla \& Paranal Observatory & \\
\hline TGIR & $\mathrm{CFHT}$ & Canada-France-Hawaii Telescope & \\
\hline TGIR & IRAM & Institut de RadioAstronomie Millimétrique & \\
\hline IR & CDS & Centre de Données astronomiques de Strasbourg & \\
\hline IR & ESO-INSTRUM & $\begin{array}{l}\text { Instrumentation pour les grands télescopes } \\
\text { de l'ESO }\end{array}$ & \\
\hline IR & $H_{E S S}{ }^{10}$ & High Energy Stereoscopic System & \\
\hline IR & ILT-LOFAR FR & International Low Frequency Radio Array Telescope & \\
\hline Projet & $C T A^{11}$ & Cherenkov Telescope Array & CTA (2008) \\
\hline
\end{tabular}

\section{PHYSIQUE NUCLÉAIRE ET DES HAUTES ENERGIES}

\begin{tabular}{|c|c|c|c|}
\hline Ol & CERN-LHC & $\begin{array}{l}\text { Organisation Européenne pour la Recherche } \\
\text { Nucléaire - Grand Collisionneur Hadronique/ } \\
\text { European Organization for Nuclear Research - } \\
\text { Large Hadron Collider }\end{array}$ & \\
\hline TGIR & EGO-VIRGO & $\begin{array}{l}\text { Observatoire Européen Gravitationnel-VIRGO/ } \\
\text { European Gravitational Observatory - VIRGO }\end{array}$ & \\
\hline TGIR & FAIR & Facility for Antiproton and Ion Research & FAIR (2006) \\
\hline TGIR & GANIL-Spiral2 & $\begin{array}{l}\text { Grand Accélérateur National d'lons Lourds - } \\
\text { Système de production d'Ions Radioactifs en Ligne } \\
\text { de } 2^{\mathrm{e}} \text { génération }\end{array}$ & Spiral2 (2006) \\
\hline IR & $H^{H} S S^{12}$ & High Energy Stereoscopic System & \\
\hline IR & KM3NeT & Kilometre Cube Neutrino Telescope & $\begin{array}{l}\text { KM3NET (2006, } \\
\text { 2016) }\end{array}$ \\
\hline IR & LSST & Large Synoptic Survey Telescope & \\
\hline Projet & $C T A^{13}$ & Cherenkov Telescope Array & CTA (2008) \\
\hline
\end{tabular}

\footnotetext{
9 Relève également du secteur "Sciences Humaines et Sociales".

10 Relève également du secteur « Physique nucléaire et des hautes énergies ».

11 Relève également du secteur " Physique nucléaire et des hautes énergies ».

12 Relève également du secteur « Astronomie et Astrophysique ».

13 Relève également du secteur " Astronomie et Astrophysique ».
} 


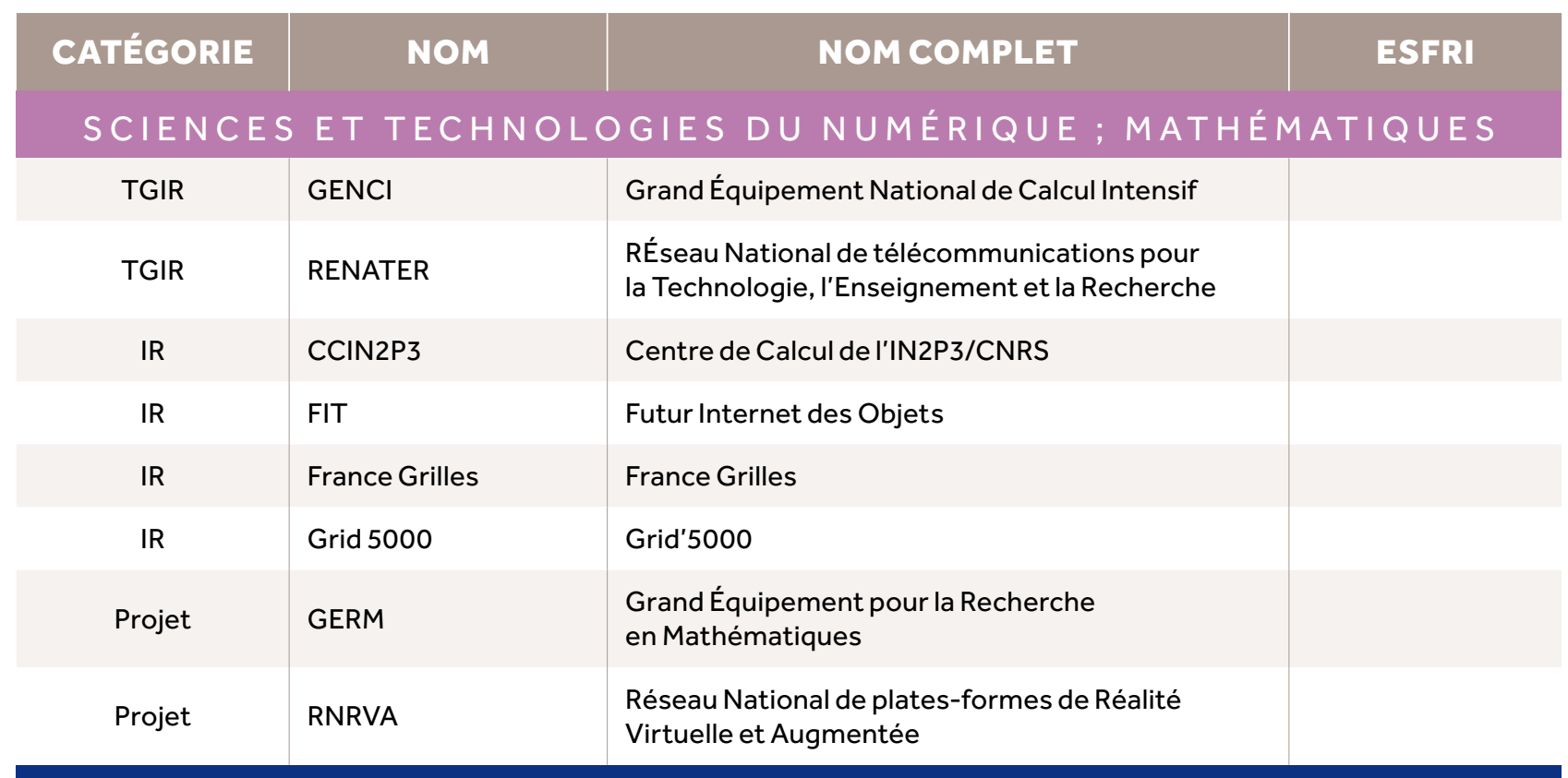

\section{INFORMATION SCIENTIFIQUE ET TECHNIQUE}

\begin{tabular}{|c|c|c|}
\hline IR & COLLEX PERSEE & $\begin{array}{l}\text { Collections d'excellence pour la Recherche - } \\
\text { Persée }\end{array}$ \\
\hline IR & HAL, CCSD & $\begin{array}{l}\text { Archive ouverte HAL (Hyper Article en Ligne), } \\
\text { Centre pour la Communication Scientifique Directe }\end{array}$ \\
\hline IR & NUMEDIF & $\begin{array}{l}\text { NUMérique pour l'ÉDItion et la DIFFusion } \\
\text { de la production scientifique }\end{array}$ \\
\hline$I R$ & OpenEdition ${ }^{14}$ & $\begin{array}{l}\text { Plateformes de publication électronique } \\
\text { en sciences humaines et sociales }\end{array}$ \\
\hline
\end{tabular}

14 Relève également du secteur «Sciences Humaines et Sociales ». 


\section{CRÉDITS PHOTOS}

\section{COUVERTURE}

K. Pierre / IPEV; Gabriel OLALDE/CNRS Photothèque; (c) C2RMF/A. Maigret-V. Fournier; MRSH-communication-2015; ( ) Pascal Morillon/Université de Rennes 1 ; (c) Cyril FRESILLON/CNRS Photothèque; Philippe Stroppa; Gemini-CFHT ; @ Julien Fuchs/LULI.

\section{SCIENCES HUMAINES ET SOCIALES}

Page d'ouverture Fotolia

ERIHS $\odot$ C2RMF/A. Maigret-V. Fournier

HUMANUM Stéphane Pouyllau, CNRS, 2015

PROGEDO @ PROGEDO

RNMSH @ Reseau National MSH - 2014

\section{SCIENCES DU SYSTEMME TERRE ET DE L'ENVIRONNEMENT}

Page d'ouverture Guillaume Petriat

ACTRIS Thibaut VERGOZ/OSU Réunion/CNRS Photothèque

AnaEE Copyright Quentin Benard

CEPMMT ECMWF

Concordia K. Pierre / IPEV

CLIMERI @ Animea, F. Durillon pour le LSCE / CEA / IPSL

Constances INSERM/Patrice Latron

ECORD ACEX1_M-Jakobsson et ACEX2_M-Jakobsson : M. Jakobsson $\odot$ ECORD/IODP

ECOSCOPE CNRS photothèque - Yann Fontana, Dominique Gibert, Emmanuel Perrin; INRA photothèque - Frédérique Bressoud, Rémi Le Bastard; Droits réservés

ECOTRONS Atelier d'Architecture Téqui/J. Roy

EMPHASIS Claude Welcker $\odot$ INRA Montpellier

EMSO $\odot$ EMSO

Euro-Argo. ERIC Euro-Argo

FOF UMS Flotte océanographique française

I-LI-CO Ifremer, CNRS, SHOM, UBO

IAGOS T. Laurent

ICOS Les Défis du CEA/F. Mathé

OZCAR Jerome Gaillardet @ IPGP

Pôle de données ESA/ATG Medialab et MétéoFrance

RARe INRA

RECOLNAT e-ReCoINat-MNHN

RESIF-EPOS CNRS

SAFIRE SAFIRE, Jean-Christophe Canonici

\section{ÉNERGIE}

Page d'ouverture $\odot$ Fotolia

ECCSEL BRGM / BLCom

FR-Solaris Gilles Flamant

SOPHIRA M. Joanny-CEA.

THEOREM M. Nicolas JOB

WEST CEA-IRFM- Christophe Roux

\section{BIOLOGIE ET SANTÉ}

Page d'ouverture $\odot$ CNRS/Denis Morel

Biobanques istock

Celphedia-Phenomin @ INSERM

Celphedia-Tefor TEFOR Core Facility

Constances INSERM/Patrice Latron

CRB-ANIMINRA

E-CELL C. Jorgensen @ U1183 Inserm

ECOSCOPE CNRS photothèque - Yann Fontana, Dominique Gibert, Emmanuel Perrin; INRA photothèque - Frédérique Bressoud, Rémi Le Bastard; Droits réservés

EMBC EMBC

EMBL EMBL_Lignes_Lumiere_Octobre_2015_Pierre Jayet_2-2

EMBRC $\odot$ W. Thomas

F-CRIN $\odot$ F-CRIN 2016

FBI $\odot$ Julien Burger - Institut Jacques Monod

FLI FLI

Fr-Hadron JC Blouzard détenteur

FRISBIIGBMC

France-Génomique $\odot$ Francis Rhodes

HIDDEN Inserm/Delapierre, Patrick

IBISBA @ LISBP

IDMIT Tourret-Jonery Architectes

IFB JFGibrat

INGESTEM @ Inserm U935/ESTeam Paris Sud, O. Féraud.

METABOHUB L. Lizet

NEURATRIS @NeurATRIS/CEA

PGT-2 Plateforme GenoSafe @ Christophe Hargoues

PROFI INSERM/Patrice Latron 


\section{SCIENCES DE LA MATIËRE ET INGÉNIÉRIE}

Page d'ouverture @ Jérémy BARANDE/Permission to use granted by Newport Corporation

EMIR @ Serge Bouffard

ILLILL_R.Cubitt

LLB P. Stroppa/CEA

LNCMI @ Julien Billette - LNCMI @ Denis Morel - Photothèque CNRS

PETAL CEA

RENARD crédit IM2NP

RMN $\odot$ CNRS

FT-ICR Edith Nicol

LULI-2 @ Julien Fuchs/LULI

METSA @ Pascal Didier/CNRS/IPCMS

SOLEIL Synchrotron SOLEIL - CAVOK Production/Laurent PERSIN

RENATECH @ Cyril FRESILLON/CNRS Photothèque

XFEL (xfel.riken.jp/eng/index.html) $\odot \mathrm{X}$-ray Free Electron Laser

ESRF 2 ESRF/P. Ginter

ESS European Spallation Source

\section{ASTRONOMIE ET ASTROPHYSIQUE}

Page d'ouverture $\odot$ Clem \& Adri Bacri-Normier/ESO

CDS $\odot 2010$ UDS/CNRS by CDS - Distributed under CNU CPL V.3

CFHT Jean-Charles Cuillandre (CEA-Saclay - Obs. de Paris - CFHT)

ESO ALMA A. Marinkovic/X-Cam/ALMA (ESO/NAOJ/NRAO)

CTA ๑ G. Pérez, IAC (SMM)

ESO-EELT ESO/L. Calçada

ESO-INSTRU ESO

ESO - LSP J.L. Dauvergne \& G. Hüdepohl (atacamaphoto. (com)/ESO

ESO-OIESO-OI

HESS Christian99 - Travail personnel, CC BY-SA 3.0

ILT Copyright belongs to ASTRON (NL)

IRAM @ IRAM

\section{PHYSIQUE NUCLÉAIRE ET DES HAUTES ÉNERGIES}

\section{Page d'ouverture $\odot$ CERN}

CERN CERN

EGO-VIRGO C. Fresillon/VIRGO/CNRS Photothèque

FAIR ion42 for FAIR/GSI Helmholtzzentrum für Schwerionenforschung

GANIL GANIL

Km3NET Propriété KM3NeT Collaboration

LSST Large Synoptic Survey Telescope (LSST)

\section{SCIENCES ET TECHNOLOGIES DU NUMÉRIQUE; MATHÉMATIQUES}

Page d'ouverture Cyril FRESILLON/CNRS Photothèque CCP-IN2P3 @ A.Melliez/CC-IN2P3 (CNRS)

FIT UPMC

France-Grilles $\odot$ France Grilles - Silvia Gervois

GENCI $\odot$ Cines

GERM $\odot$ GERM

GRID-5000 @ Pascal Morillon/Université de Rennes 1

RENATER RENATER

RNRVA $\odot$ Bruno Arnaldi

\section{INFORMATION SCIENTIFIQUE ET TECHNIQUE}

Page d'ouverture @ SCY/CC.O/Pixabay

COLLEX Jean-Pierre Rosenkranz pour BNU Strasbourg; UMS Persée

HAL $\odot$ CCSD

NUMEDIF MRSH-communication-2015

OpenEdition Lisa George (Cléo). Licence CCBY 


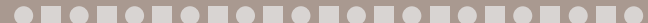

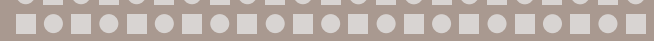

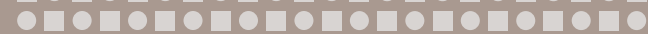

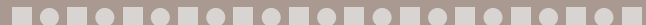
onomononononomonononomo moromomomomoromomomomo Mel'Enseignement supérieur et de la Recherche

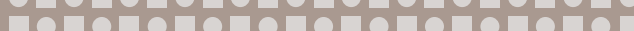
1 , rue Descartes 75231 Paris cedex 05

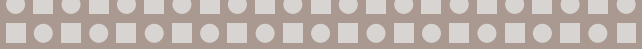

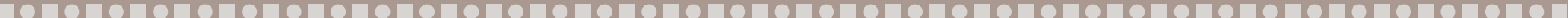

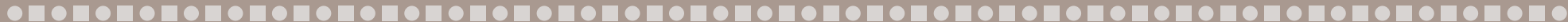

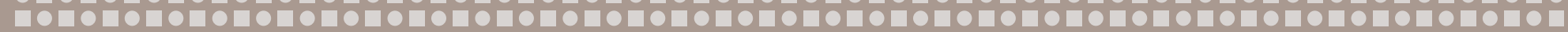

